\title{
Control, Simulation, and Testbed Development for Improving Maritime Launch and Recovery Operations
}

by

\author{
Johanna McPhee
}

A thesis submitted to the Faculty of Graduate and Postdoctoral Affairs in partial fulfillment of the requirements for the degree of

\author{
Masters of Applied Science \\ in \\ Mechanical Engineering
}

Department of Mechanical and Aerospace Engineering

Carleton University

Ottawa, Ontario

(C) 2019

Johanna McPhee 


\section{Abstract}

Improvements to launch and recovery operations at sea are driven by the desire to increase safety and operational availability. This thesis presents various tools to improve motion compensation strategies in maritime launch and recovery: a 3D computer simulator to examine wave synchronization, a signal prediction algorithm for Go-NoGo states and a hardware set-up to simulate ship and wave motion.

The 3D simulator of towed body dynamics was advanced to model the wave interactions with the cable and towed body as the body exits the water. Small scale simulations were run to investigate the inclusion of wave synchronization in established active heave compensation strategies where the hypothesis that wave synchronization would reduce variations in cable tension was not supported; the simulations demonstrated that wave synchronization increased variations in cable tension compared to simulations not using motion compensation. The use of a signal prediction method that forecasts a periodic signal based only on historic data of the signal was explored. The method is a means to predict safe breach events where the prediction algorithm was advanced and tuned to determine Go-NoGo states. A Go scenario identified by the mean and one standard deviation of the predicted signal was found to produce a Go-NoGo signal that agreed most with the desired Go-NoGo signal for forecasts up to $10 \mathrm{~s}$. For the development of laboratory equipment for the Carleton University flume tank, a ship motion simulator was designed and built to emulate 5 degrees-of-freedom of ship motion and a kinematic analysis was performed to characterize the system workspace. For producing waves in the flume tank, a design methodology was developed for the design of a plunger-type wavemaker. A numerical model for determining the wave amplitude to actuator stroke length ratio was advanced to include the effects of a flow current. The design methodology enables the designer to select an appropriate actuator and plunger shape based on an operating point that incorporates multiple design variables. 
To my parents, who set the bar high and fueled my curiosity.

To my siblings, Emma and Aaron, who shared my love of reading and always reminded me to pack a notebook and pencil before heading out the door. 


\section{Acknowledgements}

First and foremost I would like to express my sincere gratitude to my advisor Dr. Rishad Irani for his guidance, encouragement and support throughout this thesis work. Additionally, I would like to thank my committee members Dr. John Hayes, Dr. Fidel Khouli and Dr. Huseyin Gunay for serving on my committee and providing valuable comments and suggestions.

I would also like to thank Alex Proctor, Kevin Sangster and Ian Lloy of the Carleton University Mechanical and Aerospace Engineering Machine Shop for their support in fabricating parts for the lab equipment used in this thesis work.

To my fellow lab members of the Multi-Domain Laboratory: Cassidy Westin, the first member of our group who set high standards; Shadi Abujoub, for letting me have a small part in his research endeavours; Ryan McKenzie, the LabVIEW wizard whose support with developing LabVIEW code throughout this work cannot be understated; and Iain Martin and Stephanie Lowell for their helpful input.

I would also like to thank the Natural Sciences and Engineering Research Council of Canada (NSERC) and Kraken Robotic Systems Inc. for their funding and support of this thesis. 


\section{Contents}

Abstract $\quad$ i

Acknowledgements

List of Figures $\quad$ xiv

List of Tables $\quad$ xvi

Nomenclature $\quad$ xvii

1 Introduction 1

2 Background and Literature Review 6

2.1 Existing Equipment . . . . . . . . . . . . . . 6

2.2 Emulating Ship Motion . . . . . . . . . . . . . . . . 8

2.3 Modelling Ocean Waves . . . . . . . . . . . . . . . . . 9

2.3.1 Regular Waves . . . . . . . . . . . . . . . . . . . . 9

2.3.2 Irregular Waves . . . . . . . . . . . . . . . . 13

2.3.3 Sea States . . . . . . . . . . . . . . . 15

2.4 Wave Generators . . . . . . . . . . . . . . . . . . . . 18

2.4.1 Piston-type Wavemakers . . . . . . . . . . . . . . . . . 19

2.4.2 Flap-type Wavemakers . . . . . . . . . . . . . . . . 19 
CONTENTS

2.4.3 Plunger-type Wavemakers . . . . . . . . . . . . . . . 20

2.5 Wave Synchronization . . . . . . . . . . . . . . . . 22

2.6 Summary . . . . . . . . . . . . . . . . . . 27

3 Wave Synchronization Model and Simulation 29

3.1 Towed body simulator overview . . . . . . . . . . . . . . 30

3.2 Wave modeling and ship motion . . . . . . . . . . . . . . . 32

3.3 External forces . . . . . . . . . . . . . . . . . 34

3.3.1 Breach ..................... 36

3.4 Compensator strategies . . . . . . . . . . . . . . . . 40

3.5 Simulation results . . . . . . . . . . . . . . . . . . . . 42

3.6 Summary . . . . . . . . . . . . . . . . . . . 52

4 Advancing a Wave Prediction Algorithm for Breach Condition Forecasts $\quad 54$

4.1 Signal Prediction Algorithm . . . . . . . . . . . . . . . 55

4.2 Benchmark Data . . . . . . . . . . . . . . . . 58

4.3 Mode Detection Tuning ................. 58

4.4 Prediction Algorithm Simulation Results . . . . . . . . . . . . . 61

4.5 Developing the Go/NoGo Criteria . . . . . . . . . . . . 64

4.6 Go-NoGo Command Latching . . . . . . . . . . . . . . . . 67

4.7 Summary . . . . . . . . . . . . . . . . . 69

5 Development and Quantification of a Wave Generator and Ship Motion Simulator $\quad 72$

5.1 Theoretical analysis of a plunger-type wavemaker . . . . . . . . 73

5.1.1 Plunger-type wavemaker boundary value problem . . . . . . . 74 
CONTENTS

5.1.2 Investigating the effects of current on wavemaker design . . . 80

5.2 Conceptual design of a plunger-type wavemaker . . . . . . . . . . 83

5.3 Design overview of a robotic ship motion simulator . . . . . . . 85

5.4 Kinematic analysis and control scheme . . . . . . . . . . . . 87

5.4.1 Inverse kinematics of a parallel manipulator . . . . . . . . . . 89

5.4 .2 Workspace analysis . . . . . . . . . . . . . . . 92

5.5 Preliminary experimental validation of a ship motion simulator system 103

5.5.1 Control and trajectory planning of the ship motion simulator . 103

5.5.2 Experimental results ................ 106

5.6 Summary . . . . . . . . . . . . . . . . . 107

6 Conclusions and Future Work 109

6.1 Objective $1 \ldots \ldots \ldots \ldots$. . . . . . . . . . . . . . . 110

6.2 Objective $2 \ldots \ldots \ldots \ldots \ldots$

6.3 Objective $3 \ldots \ldots \ldots \ldots \ldots$. . . . . . . . . . . . . . . . . . . .

6.4 Objective $4 \ldots \ldots \ldots \ldots \ldots$. . . . . . . . . . . . . . . . . . . . . . .

6.5 Future Work . . . . . . . . . . . . . . . . 112

6.6 List of Publications . . . . . . . . . . . . . . . . . 113

$\begin{array}{lr}\text { References } & 114\end{array}$

$\begin{array}{ll}\text { A Additional Figures } & 121\end{array}$

B Technical Drawings $\quad 128$ 


\section{List of Figures}

1.1 Schematic of a ship and towed-body in a lifting operation. . . . . . . 2

1.2 The three stages of a recovery operation: 1) operation, 2) breach and 3) suspended. . . . . . . . . . . . . . . . . . . 3

2.1 Schematic of the water tunnel at Carleton University. . . . . . . . . . 7

2.2 Reference frame for the six degrees-of-freedom of ship motion. Positive rotations about each axis are counterclockwise. . . . . . . . . . 8

2.3 Schematic of the boundary value problem for a two-dimensional water wave. . . . . . . . . . . . . . . . . . . . . 10

2.4 Bretschneider wave energy spectra. . . . . . . . . . . . . . 14

2.5 Bretschneider spectrum with sea states. . . . . . . . . . . . . 17

2.6 Schematic of a laboratory scale piston-type wavemaker. . . . . . . . 19

2.7 Schematic of a laboratory scale a) rigid flap and b) flexible flap type wavemaker. . . . . . . . . . . . . . . . 20

2.8 Schematic of a laboratory scale plunger-type wavemaker. . . . . . . . 21 


\section{LIST OF FIGURES}

2.9 Schematic of a laboratory scale moonpool with definition of the coordinate system. . . . . . . . . . . . . . . . . . 23

3.1 Schematic of the towed body simulator system. The $y$-axis is directed into the page. . . . . . . . . . . . . . . .

3.2 Configuration of tow cable linkages connected by a universal joint with respective co-ordinate frames. Adapted from $[1] \ldots . . . . . . .$.

3.3 Resolved 3 degrees of freedom ship motion data scaled to $\pm 4 \mathrm{~cm}$ from the Australian DSTO report. Reproduced from $[1]$. . . . . . . . .

3.4 Change in submerged volume and wetted area of the towed body as the body is removed from the water during breach. Geometry is shown for forces acting on the towed body in the $x-z$ plane and is the same for forces acting in the $y-z$ plane. . . . . . . . . . . . . . . .

3.5 Parameters used in the derivation of volume of a partially submerged sphere where $h \epsilon\left[0,2 r_{T B}\right]$ and $x=\sqrt{r_{T B}^{2}-\left(r_{T B}-h\right)^{2}} \ldots \ldots$

3.6 Front and bottom views displaying the change in wetted area of the towed body. The bottom view is projected onto the $x-y$ plane that is perpendicular to the flow in the $z$ direction. . . . . . . . . . .

3.7 Waterline set-point algorithm with wave synchronization. . . . . . .

3.8 Sheave set-point algorithm with wave synchronization. . . . . . . .

3.9 Mean cable tension measured during simulations that were run with the combined wave synchronization and $\mathrm{AHC}$ strategies compared to the simulations run with no control for sea states a)3, b)4, c)5 and d)6. Error bars represent 2 standard deviations. . . . . . . . . . 


\section{LIST OF FIGURES}

3.10 Mean cable tension measured during simulations that were run with the combined wave synchronization and AHC strategy compared to the simulations run with only heave compensation for sea states a) 3 , b) 4, c) 5 and d)6. Error bars represent 2 standard deviations. . . . . . 46

3.11 Adapted from Sagutun et al. [2] : Experimental results with regular waves at $T_{p}=1.25 \mathrm{~s}$ and $H_{s}=1.8 \mathrm{~cm}$, spherical payload. For the wire tension, we show both raw data (thin lines) and low-pass filtered data (thick lines). The payload position (measured using the motor shaft encoder) is in a vessel-fixed coordinate frame with zero at mean sea level. 48

3.12 Simulated results with regular waves at SS6, $T_{p}=1.01 \mathrm{~s}$ and $H_{s}=$ $0.035 \mathrm{~m}$. Wire tension is shown on the left for a) combined heave compensation and wave synchronization, c) heave compensation only and e) no control. The towed body position in the world frame $z$ direction is shown on the right for b) combined heave compensation and wave synchronization, d) heave compensation only and f) no control. The towed body breach point is indicated by a hexagram in the towed body position figures. . . . . . . . . . . . . . .

3.13 Simulated results with regular waves at SS3, $T_{p}=0.63 \mathrm{~s}$ and $H_{s}=$ $0.007 \mathrm{~m}$. Wire tension is shown on the left for a) combined heave compensation and wave synchronization, c) heave compensation only and e) no control. The towed body position in the world frame $z$ direction is shown on the right for b) combined heave compensation and wave synchronization, d) heave compensation only and f) no control. The towed body breach point is indicated by a hexagram in the towed body position figures. . . . . . . . . . . . . . . . 


\section{LIST OF FIGURES}

3.14 Simulated results for the towed body position with combined heave compensation and wave synchronization during a recovery operation with irregular waves at SS6. The towed body breach point is indicated by a hexagram. . . . . . . . . . . . . . . . . .

3.15 Simulated results for the towed body position with combined heave compensation and wave synchronization during a recovery operation with irregular waves at SS3. The towed body breach point is indicated by a hexagram. . . . . . . . . . . . . . . . . . 52

4.1 Structure overview of the prediction method and Go-NoGo discriminator. 58

4.2 Benchmark test cases of resolved ship motion from the Australian

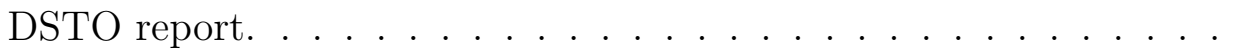

4.3 Measured and predicted signal together, with prediction signal time shifted to the input signal timeframe. The prediction discontinuity due to no modes detected is shown in green. . . . . . . . . . . . 60

4.4 Dominant peaks detected from the FFT spectrum of the first $10 \mathrm{~s}$ of the benchmark case for $\mu=0.01 . \ldots \ldots \ldots$

4.5 Dominant peaks detected from the FFT spectrum of the first $10 \mathrm{~s}$ of the benchmark case for $\mu=0.1 \ldots \ldots \ldots$

4.6 SPA function block implemented in the Simulink environment. . . . .

4.7 Benchmark data with prediction curve at $t=38$ s plotted over a continuous time horizon of $10 \mathrm{~s}$ into the future. . . . . . . . . .

4.8 Error between benchmark data and prediction at a) 1 s into the future, b) $3 \mathrm{~s}$ into the future and c) $10 \mathrm{~s}$ into the future. . . . . . . . 


\section{LIST OF FIGURES}

4.9 Go-NoGo command signals with desired command signal in a), and resulting command outputs for approaches b) MPT, c) 1-SD and d) 1.645-SD. For display purposes, the results are shown for $t=50 \mathrm{~s}$ to $t$

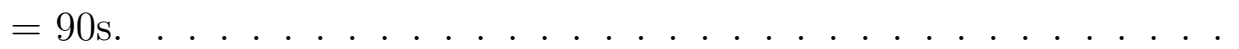

4.10 Summary of "Go time" error between desired amount of time a GO state should be output and the actual amount of time a GO state is output for all six benchmark test cases. . . . . . . . . . . . .

4.11 Flow chart of the logic used in the command signal latching algorithm. 68

4.12 Go-NoGo command signals for the benchmark test case without latching in a) and with command latching in b). For display purposes, the results are shown for $t=50 \mathrm{~s}$ to $t=90 \mathrm{~s} . \ldots \ldots$

5.1 Schematic of the plunger-type wavemaker problem. . . . . . . . . . . 74

5.2 Amplitude to stroke ratio vs dimensionless frequency parameter $k_{p} b$ for various wedge shapes where $\mathrm{d}=0.5 \mathrm{~m}$ and $\mathrm{h}=1.0 \mathrm{~m}$. . . . . . 78

5.3 Graphical representation of the dispersion relationship for the standing wave modes. Here, $\omega=7.4 \mathrm{rad} / \mathrm{s}$ and $\mathrm{h}=71 \mathrm{~cm} . \ldots . . . .$.

5.4 Relationship between a uniform current and the wave amplitude to stroke ratio of a plunger-type wavemaker. Here, $\omega=7.4 \mathrm{rad} / \mathrm{s}$ and $\mathrm{h}$ $=1 \mathrm{~m} \ldots \ldots \ldots \ldots$

5.5 Amplitude to stroke ratio vs dimensionless frequency parameter $k_{p} b$ for various wedge shapes and current $U_{o}$ where $\mathrm{h}=71 \mathrm{~cm}$ and $T=0.85 \mathrm{~s}$.

5.6 Conceptual design of a plunger-type wavemaker for the Carleton University flume tank. . . . . . . . . . . . . . . . . . 


\section{LIST OF FIGURES}

5.7 CAD model representation (left) and physical assembly (right) of the robotic ship motion simulator designed and built for the Carleton University flume tank. . . . . . . . . . . . . . . . .

5.8 Top view representation of the surge-sway gantry system with a) showing initial positions of both axes and b) showing the end position of $200 \mathrm{~mm}$ on both axes. . . . . . . . . . . . . . . . .

5.9 Isometric view representation of the 3-DOF motion platform. Note: support structure and the surge-sway gantry system have been removed for clarity. . . . . . . . . . . . . . . . . .

5.10 Kinematic model for a 3-DOF parallel kinematic manipulator. . . .

5.11 Workspace of the surface points $P_{1}, P_{2}, P_{3}$ and origin $O_{P} . \ldots 94$

5.12 Workspace of the surface points $P_{1}, P_{2}, P_{3}$ and origin $O_{P}$ in the base frame Cartesian planes a)x-y, b)x-z and c)y-z. . . . . . . . . . . 96

5.13 Representations of a $20 \mathrm{deg}$ roll angle on the 3-DOF motion platform. 97

5.14 Representations of a 20 deg pitch angle on the 3-DOF motion platform. 98

5.15 U-joint offset $k$ from the platform. . . . . . . . . . . . . . . . 98

5.16 Platform position correction due to offset $k$. . . . . . . . . . . . 99

5.17 Actuator extension correction for the $i^{t h}$ leg. . . . . . . . . . . . 99

5.18 Workspace of the surface points $P_{1}, P_{2}, P_{3}$ and origin $O_{P}$ corrected for the U-joint offsets.

5.19 Workspace of the surface points $P_{1}, P_{2}, P_{3}$ and origin $O_{P}$ corrected for the U-joint offsets in the base frame Cartesian planes a)x-y, b)x-z and

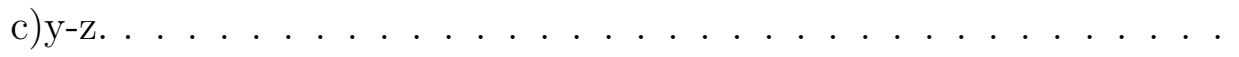




\section{LIST OF FIGURES}

5.20 Workspace of the entire motion platform, relative to the initial platform position, corrected for the U-joint offsets. . . . . . . . . . . . . . . . 102

5.21 Overview of electrical and control system components for the 5-DOF robotic ship motion simulator. . . . . . . . . . . . . . . . . . 104

5.22 Ship motion data in all degrees-of-freedom simulated using ShipMo 3D and scaled to the workspace of the ship motion simulator. . . . . . . 105

5.23 Preliminary testing results of the ship motion simulator comparing the analog inputs and actuator feedback signals. . . . . . . . . . . . . 108

A.1 Simulated results with irregular waves at SS6, $T_{p}=1.01 \mathrm{~s}$ and $H_{s}$ $=0.035 \mathrm{~m}$. Wire tension is shown on the left for a) combined heave compensation and wave synchronization, c) heave compensation only and e) no control. The towed body position in the world frame $z$ direction is shown on the right for b) combined heave compensation and wave synchronization, d) heave compensation only and f) no control. The towed body breach point is indicated by a hexagram in the towed body position figures. . . . . . . . . . . . . . . . . . . . 122

A.2 Simulated results with irregular waves at SS3, $T_{p}=0.63 \mathrm{~s}$ and $H_{s}$ $=0.007 \mathrm{~m}$. Wire tension is shown on the left for a) combined heave compensation and wave synchronization, c) heave compensation only and e) no control. The towed body position in the world frame $z$ direction is shown on the right for b) combined heave compensation and wave synchronization, d) heave compensation only and f) no control. The towed body breach point is indicated by a hexagram in the towed body position figures. . . . . . . . . . . . . . . . . . 123 


\section{LIST OF FIGURES}

A.3 Simulated results for the towed body position with no control during a recovery operation with irregular waves at SS3. The towed body breach point is indicated by a hexagram. . . . . . . . . . . . . . . . 124

A.4 Simulated results for the towed body position with no control during a recovery operation with irregular waves at SS6. The towed body breach point is indicated by a hexagram. . . . . . . . . . . . . . . 125

A.5 Simulated results for the towed body position with only heave compensation during a recovery operation with irregular waves at SS3. The towed body breach point is indicated by a hexagram. . . . . . . . . . 126

A.6 Simulated results for the towed body position with only heave compensation during a recovery operation with irregular waves at SS6. The towed body breach point is indicated by a hexagram. . . . . . . . . . 127 


\section{List of Tables}

2.1 Sea state definition by NATO STANAG 4194 Annex D. . . . . . . . . 16

2.2 Flume-scale sea state definition based on Froude scaling. . . . . . . . 18

2.3 Summary of existing wavemaker designs based on design criteria. . . 21

3.1 Towed body simulator system parameters [1] . . . . . . . . . . . . 31

3.2 Cable tension results for the combined wave synchronization and AHC strategy compared to simulations with no control method implemented. The mean and standard deviation of the cable tension throughout were computed on the interval $t=[0.5,15] \mathrm{s}$. All values are in $\times 10^{-2}$ Newtons. 43

3.3 Cable tension results for the combined wave synchronization and AHC strategy compared to simulations with only heave compensation applied. The mean and standard deviation of the cable tension throughout were computed on the interval $t=[0.5,15] \mathrm{s}$. All values are in $\times 10^{-2}$ Newtons. . . . . . . . . . . . . . . . .

4.1 Error metrics for various prediction horizons. . . . . . . . . . . . 64

4.2 Evaluation of GO scenario definitions. . . . . . . . . . . . 67 


\section{LIST OF TABLES}

5.1 Design requirements for a 5 DOF robotic ship motion simulator. . . . 86 


\section{Nomenclature}

\section{Greek Symbols}

$\alpha(z) \quad$ Compensator blending factor

$\beta \quad$ Wedge angle (rad)

$\epsilon \quad$ Phase angle (rad)

$\epsilon_{F F T}$ Phase angle of a dominant mode (rad)

$\epsilon_{o b s} \quad$ Adapted observer phase angle (rad)

$\eta \quad$ Position of the free surface $(\mathrm{m})$

$\hat{\eta}_{0} \quad$ Estimate of the position of the wave surface elevation (m)

$\eta_{0} \quad$ Position of of the wave surface in the body-fixed frame (m)

$\kappa(z)$ Wave decay factor

$\mu \quad$ Peak detection sensitivity parameter

$\omega \quad$ Wave frequency $(\mathrm{rad} / \mathrm{s})$

$\omega_{o} \quad$ Peak frequency of wave spectrum $(\mathrm{rad} / \mathrm{s})$

$\phi, \Phi \quad$ Velocity potential $\left(\mathrm{m}^{2} / \mathrm{s}\right)$ and Roll ( $\left.\mathrm{rad}\right)$

$\psi \quad$ Yaw $(\mathrm{rad})$ 


\section{NOMENCLATURE}

$\boldsymbol{\Psi} \quad$ Discrete system matrix

$\rho \quad$ Density $\left(\mathrm{kg} / \mathrm{m}^{3}\right)$

$\theta \quad$ Pitch $(\mathrm{rad})$

$\theta_{m} \quad$ Motor angle (rad)

\section{Roman Symbols}

a Wave amplitude (m)

$A_{F F T}$ Amplitude of a dominant mode (m)

$A_{i} \quad$ Wave amplitude of the $i^{\text {th }}$ mode (m)

$A_{\text {obs }} \quad$ Adapted observer wave amplitude (m)

$A_{p, x, y, z}$ Projected frontal area in directions $x, y, z(\mathrm{~m})$

b Mean submerged plunger width (m)

$C \quad$ Wave speed $(\mathrm{m} / \mathrm{s})$

C Output matrix

$C_{A} \quad$ Added mass coefficient

$C_{D} \quad$ Drag coefficient

$C_{e} \quad$ Water exit coefficient

$C_{s} \quad$ Slamming coefficient

d Mean submerged plunger depth $(\mathrm{m})$

$d^{\prime} \quad$ Clearance between channel bottom and plunger bottom (m)

$d_{c} \quad$ Cable diameter $(\mathrm{m})$

$d_{l} \quad$ Linear drag $(\mathrm{N} / \mathrm{m})$ 


\section{NOMENCLATURE}

$d_{s} \quad$ Damping coefficient $(\mathrm{Ns} / \mathrm{m})$

$d_{T B} \quad$ Sphere diameter $(\mathrm{m})$

$f \quad$ Frequency $(\mathrm{Hz})$

$F_{b} \quad$ Buoyancy force $(\mathrm{N})$

$F_{e} \quad$ Water exit force $(\mathrm{N})$

$f_{F F T}$ Frequency of a dominant mode $(\mathrm{Hz})$

$F_{m} \quad$ Motor force (N)

$f_{N} \quad$ Nyquist frequency $(\mathrm{Hz})$

$F_{s} \quad$ Slamming load $(\mathrm{N})$

$F_{T} \quad$ Wire tension $(\mathrm{N})$

$F_{W} \quad$ Gravitational force $(\mathrm{N})$

$f_{z} \quad$ Hydrodynamic and static force on payload (N)

$g \quad$ Gravitational acceleration $\left(\mathrm{m} / \mathrm{s}^{2}\right)$

$H \quad$ Wave amplitude (m)

$h \quad$ Water depth (m)

$h_{m} \quad$ Moonpool depth $(\mathrm{m})$

$h_{p} \quad$ Height of payload (m)

$H_{s} \quad$ Significant wave height $(\mathrm{m})$

$J_{m} \quad$ Motor inertia $\left(\mathrm{kgm}^{2}\right)$

$k \quad$ Wave number

$k_{n} \quad$ Standing wave number 


\section{NOMENCLATURE}

$k_{o} \quad$ Peak frequency $(\mathrm{Hz})$

$k_{p} \quad$ Progressive wave number

$k_{s} \quad$ Spring coefficient $(\mathrm{N} / \mathrm{m})$

L Wavelength (m)

L Observer gains matrix

$l_{i} \quad$ Length of the $i^{\text {th }}$ cable segment(m)

$L_{o} \quad$ Wavelength in deep water $(\mathrm{m})$

$L_{S P} \quad$ Cable length set point $(\mathrm{m})$

$m \quad$ Mass $(\mathrm{kg})$

$m_{C A}$ Added mass of a towed sphere $(\mathrm{kg})$

$m_{m} \quad$ Motor mass $(\mathrm{kg})$

$m_{S A}$ Added mass of a cylindrical cable segment $(\mathrm{kg} / \mathrm{m})$

$m_{s} \quad$ Pulley mass $(\mathrm{kg})$

$B \vec{q} \quad$ Translation vector from platform to the base

$R \quad$ Radius $(\mathrm{m})$

$r_{T B} \quad$ Towed body radius $(\mathrm{m})$

$S \quad$ Stroke amplitude $(\mathrm{m})$

$\hat{s} \quad$ Estimated wave motion

$S(\omega)$ Complex amplitude spectrum $\left(\mathrm{m}^{2} \mathrm{~s}\right)$

$s_{\text {Pred }}$ Predicted wave motion $(\mathrm{m})$

$T \quad$ Wave period (s) 


\section{NOMENCLATURE}

$t \quad$ Time (s)

$T^{\prime} \quad$ Period of a moving reference frame (s)

$T_{F F T}$ Fast Fourier Transform observation window (s)

$T_{m} \quad$ Motor torque $(\mathrm{Nm})$

$T_{\text {Pred }}$ Prediction horizon (s)

$T_{p} \quad$ Modal period (s)

$\bar{U}_{x, y, z}(z)$ Mean fluid flow velocities in the world frame $(\mathrm{m} / \mathrm{s})$

$U_{o, x, y, z}$ Fluid flow velocity in each direction of the body fixed coordinate frame $(\mathrm{m} / \mathrm{s})$

$U_{o} \quad$ Flow current $(\mathrm{m} / \mathrm{s})$

V Volume $\left(\mathrm{m}^{3}\right)$

$V_{\text {breach }}$ Volume of partially submerged sphere $\left(\mathrm{m}^{3}\right)$

$V_{\text {sub }} \quad$ Displaced volume $\left(\mathrm{m}^{3}\right)$

$v(t) \quad$ Static signal offset $(m)$

$V_{a} \quad$ Actuator travel speed $(\mathrm{m} / \mathrm{s})$

$v_{e} \quad$ Water exit velocity $(\mathrm{m} / \mathrm{s})$

$V_{x, y, z}$ Relative velocity between the body and fluid flow in the body fixed frame $(\mathrm{m} / \mathrm{s})$

$\hat{\mathbf{x}} \quad$ Observed model states

x $\quad$ Model states

$x, y, z$ Cartesian coordinates

$X_{B}, Y_{B}, Z_{B}$ Base Cartesian coordinate frame

$X_{P}, Y_{P}, Z_{P}$ Platform Cartesian coordinate frame 


\section{NOMENCLATURE}

$x_{T B}, y_{T B}, z_{T B}$ Towed body position in the world frame $(\mathrm{m})$

$z_{m}^{*} \quad$ Motor reference signal $(\mathrm{m})$

$Z_{\ddot{z}_{r}} \quad$ Position dependent added mass (kg)

$z_{d} \quad$ Payload reference signal $(\mathrm{m})$

$z_{m} \quad$ Motor position (m)

$z_{r} \quad$ Vertical position of payload relative to surface elevation (m)

$z_{s} \quad$ Pulley position $(\mathrm{m})$

$\hat{z}_{0} \quad$ Estimate of the vessel's vertical position $(\mathrm{m})$ 


\section{Chapter 1}

\section{Introduction}

Improvements to marine launch and recovery operations are driven by the desire to increase safety and operational availability during maritime operations. Conditions while working at sea are dangerous: wind, waves and ocean currents interact with the vessel and can cause erratic behaviour in both the vessel and its equipment. Figure 1.1 illustrates a vessel and towed body in a lifting operation. At the tip of the crane, the sheave positions the cable over the stern of the ship and the winch is used to reel-in and pay-out the cable. Launch and recovery operations often occur when the host vessel is underway. The waves interact with the host vessel causing vessel motion in six degrees-of-freedom. The ship motion imparts disturbances at the sheave, which contacts the tow cable above the water surface, and subsequently imparts the disturbance motion underwater to the towed body. Surface waves also interact with the towed body as it nears the surface during entry and extraction. Previous work in the field has advanced wave prediction methodologies and motion compensation techniques that help to decouple the motion between vessel and towed-body while mitigating risks of damage to the payload and vessel during operation.

A towed-body recovery operation is divided into three stages shown in Fig-

ure 1.2: operation (submerged), breaching and suspended. Methods for controlling 


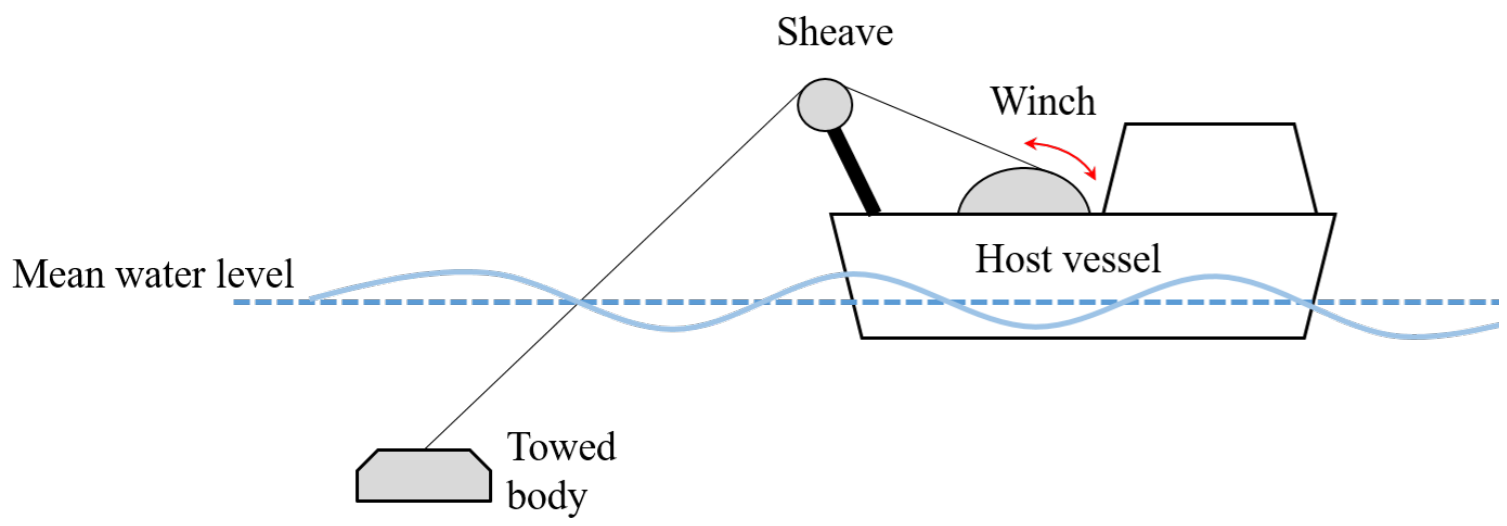

Figure 1.1: Schematic of a ship and towed-body in a lifting operation.

the payload underwater are known; previous research has developed a towed body active heave compensation (AHC) winch system that decouples the load motion from the ship heave motion [3]. In the system a winch reels-in or pays-out the cable to compensate for the vertical motion disturbance. Woodacre et al. used a model predictive controller with prediction to perform the control action of the winch [3],[4]. Knowledge of the water-to-air transition, breaching, is minimal and limited to studies performed on moonpool operations where a load is lowered into the water through an opening in the ship's hull. When breaching, the payload is subject to hydrodynamic slamming forces. In moonpool operations, wave synchronization methods have been explored by Johansen et al. [5], where the effects of slamming forces were reduced by synchronizing the payload motion with the surface wave motion at water entry. Breaching causes a pendulum motion in the air due to the removal of water drag forces during the suspended stage of recovery. Work has been done to counteract the pendulum motion by Albada et al. [6], where energy dissipation methods were investigated for a crane and winch system. Improvement to this energy dissipation method requires a sea model to obtain sea disturbances. The focus of this thesis will be on the development of tools for motion compensation during the breach phase of towed-body recovery operations.

During the breach stage, wave synchronization is a method that has been used to reduce the hydrodynamic forces on a payload as it enters and exits the water 


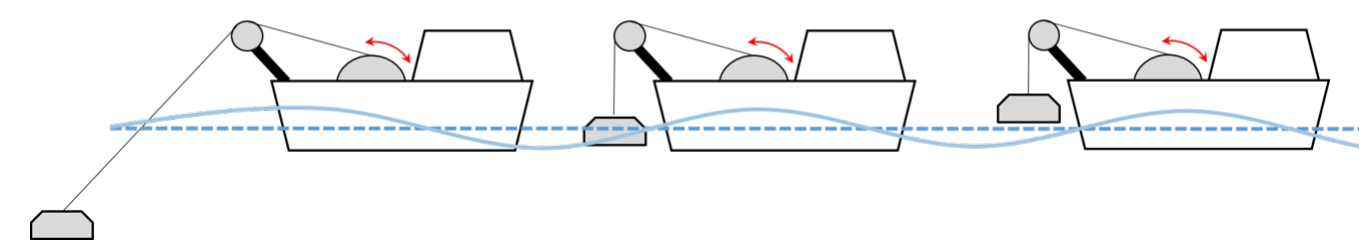

Figure 1.2: The three stages of a recovery operation: 1) operation, 2) breach and 3) suspended.

by minimizing the relative velocity between the water surface and payload. Often, wave synchronization is combined with active heave compensation for better control. Feedforward compensator strategies have been investigated where the winch motor speed setpoint is determined from the equations of motion of the motor and payload $[5,7]$. The performance of wave synchronization is evaluated by measuring the wire, or cable, tension and hydrodynamic loads on the payload. Johansen et. al performed small-scale experimental studies of combined active heave compensation and wave synchronization systems with moonpool operations that succeeded in reducing the standard deviation of the wire tension by up to $50 \%$ [5].

While the water entry, or impact, stage is well documented for moonpool operations, the water exit, or extraction, stage has not been fully studied. Furthermore, research conducted for moonpool operations is not directly transferable to over-side marine cranes, such as those that are the focus of this work, as the geometry of both systems is different and the moonpool's location within the base of the hull has a damping effect on surface waves. Therefore, the first objective of this thesis is to investigate the inclusion of wave synchronization in a set-point algorithm for the control of a winch based compensator system to reduce the hydrodynamic loads on a passive towed body during a recovery operation. A numerical model will be advanced to include the effects of waves on a towed body as it is recovered from the water. Simulations will be performed to evaluate the performance of the wave synchronization compensator strategy. 
Experimental and numerical model comparisons will be performed in the flume tank at Carleton University where both wave motion and ship motion must be emulated. However, the flume tank laboratory is not equipped to generate waves nor simulate ship motion. Therefore the second objective of this thesis is to develop and quantify a robotic ship motion simulator for the flume tank laboratory at Carleton University. A flume-scale ship motion simulator will be created to emulate ship motion based on scaled IMU data acquired at sea. Additionally, the third objective of this thesis is to propose a design for a wave generator capable of creating waves in the flume tank. The water channel conditions will be evaluated and a conceptual design with all associated wavemaker theory will be provided for the flume tank conditions.

As a final objective of this thesis, the fidelity of a wave prediction algorithm will be investigated as it applies to forecasting future breach conditions in any sea state. Short term signal prediction is already used in active heave compensation strategies [3]. In addition to objective one, the use of long term signal prediction of ship motion to determine when a towed body should commit to breach within motion thresholds will be studied. As part of this objective Go-NoGo states will be developed and examined. In future work, the definition of a safe breach condition will be investigated.

The key contributions of the research shown in this thesis are:

1. The inclusion of waves in a computer simulator that models towed body and cable behaviour.

2. An investigation of the usefulness of wave synchronization in motion compensator strategies in a recovery operation.

3. An investigation of the use of a wave prediction algorithm for forecasting longterm future breach conditions.

4. The development of important laboratory equipment for the water channel test facility at Carleton University. 


\section{CHAPTER 1. INTRODUCTION}

This thesis consists of six chapters. Chapter 2 contains a literature review of existing literature concerning the development of water channel laboratory equipment including a review of wave modeling methods for regular and irregular waves, and a overview of the the existing water channel facility at Carleton University. Wave synchronization is also introduced. Chapter 3 describes the process of advancing a computer simulator of a cable and towed body in a towing operation such that the simulator can also be used to simulate towed body and cable behaviour in a recovery operation. The heave compensation strategies are amended to include wave synchronization and simulation results for this described compensation method are also discussed. In Chapter 4, a wave prediction algorithm is advanced for use with forecasting breach conditions and develops a Go-NoGo methodology. Chapter 5 examines the testbed which was developed in this thesis work, describing in detail the design of a flume scale wave generator and robotic ship motion simulator. A workspace analysis is provided for the ship motion simulator and the results of preliminary testing of the

simulator are presented. Contributions of this thesis are summarized in Chapter 6 and potential improvements are suggested for future work. 


\section{Chapter 2}

\section{Background and Literature Review}

The work conducted for this thesis required the development of sophisticated lab equipment necessary to emulate various sea environments. In this chapter, the design specifications for the laboratory equipment are presented along with a review of the literature that was consulted during the design process. Section 2.1 first reviews the existing equipment at Carleton University followed by an overview of the ship geometry and requirements for the ship motion simulator in Section 2.2. In Section 2.3, a review of the literature for modeling regular and irregular waves is discussed followed by an overview of common types of wave generators in Section 2.4. Past research conducted in wave synchronization is explored in Section 2.5. Finally, a chapter summary is provided in Section 2.6.

\subsection{Existing Equipment}

The flow visualization water tunnel at Carleton University is primarily used for conducting continuous flow tests that demonstrate fluid dynamic phenomena. The water channel, depicted schematically in Figure 2.1, consists of an inflow delivery section, contraction section, test section and discharge section. Flow is circulated by first 


\section{CHAPTER 2. BACKGROUND AND LITERATURE REVIEW}

entering the delivery section. It then passes through the contraction section where it passes through honeycomb panels that straighten the flow to the test section. The flow then exists the test section into the discharge section where it is recirculated to the delivery section. Flow is circulated at a flow rate up to 2,800 gallons/minute $\left(0.18 \mathrm{~m}^{3} / \mathrm{s}\right)$ to provide up to $1.0 \mathrm{ft} / \mathrm{sec}(0.305 \mathrm{~m} / \mathrm{s})$ flow rate in the test section [8]. The test section consists of tempered glass for viewing with dimensions 24 in (0.61
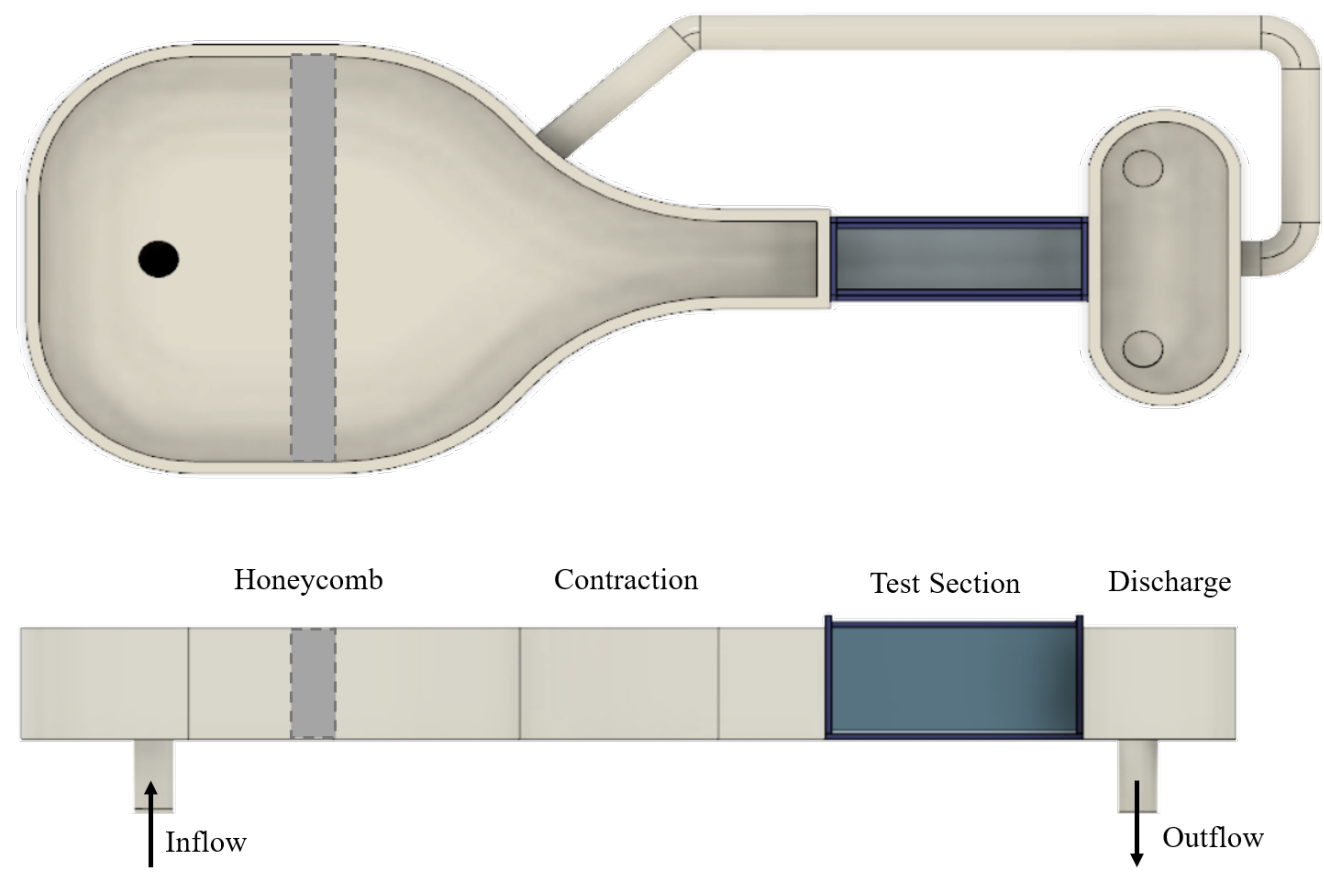

Figure 2.1: Schematic of the water tunnel at Carleton University.

m) wide, 72 in $(1.8 \mathrm{~m})$ long and 36 in $(0.91 \mathrm{~m})$ high. Water depth $h$ in this section is adjustable to 2 in $(0.05 \mathrm{~m})$ below the top of the section walls.

For experimental testing, the ship motion simulator, which will be designed and constructed as part of this thesis, will operate in the test section. The wave generator, for which a conceptual design will be suggested within this work, must be mounted upstream of the ship motion simulator. Dean and Dalrymple [9] recommend that waves generated by a wave generator be fully developed progressive waves by the time the waves propagate to the area of interest, which for this work is the flume tank test section. Chapter 5 will investigate how far the wave generator must be mounted 
upstream in order to meet this criteria.

\section{$2.2 \quad$ Emulating Ship Motion}

Figure 2.2 illustrates the two coordinate frames used to describe ship orientation. The $x, y$ and $z$ axes represent the inertial reference frame. The body-fixed frame, located at the centre of gravity $(\mathrm{CoG})$ of the ship, is represented by the $X, Y$, and $Z$ axes. The position of the ship CoG is defined by the vector $\mathbf{p}_{C o G}$ in the inertial reference frame. In the body-fixed frame, surge, sway and heave motion are along the axes $X$, $Y$, and $Z$ respectively. Yaw, pitch and roll are the three Euler angles $\psi, \theta$ and $\phi$. By convention, positive rotation is determined by the right-hand rule about each axis.

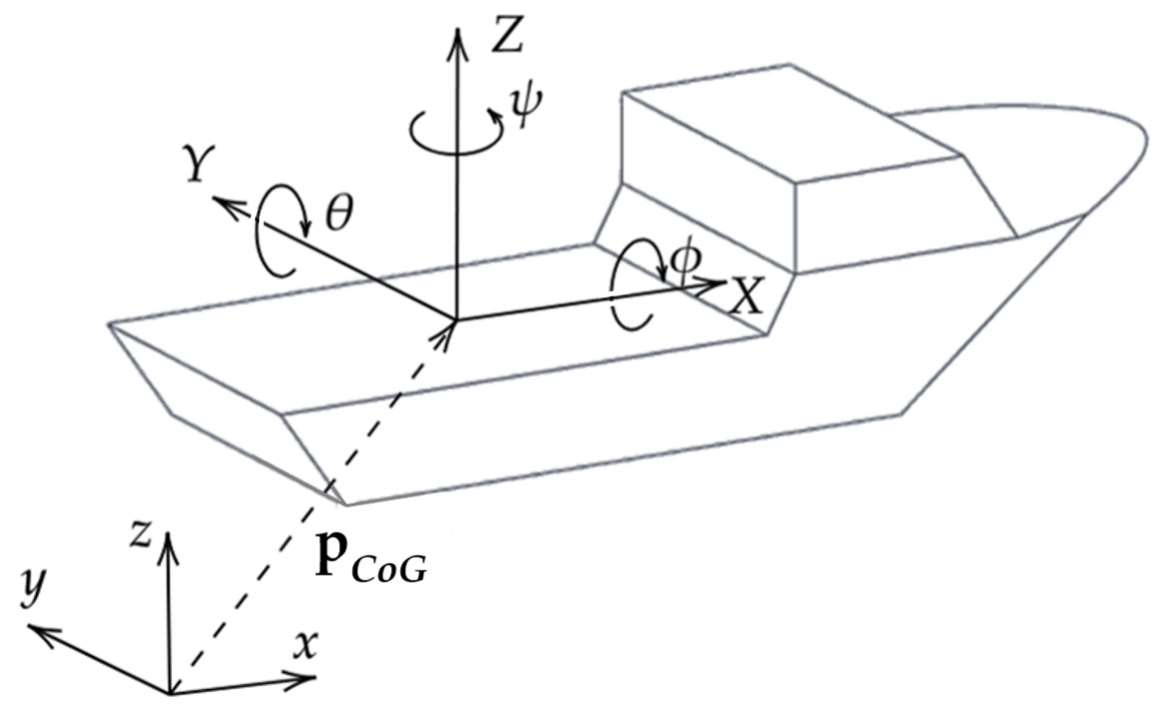

Figure 2.2: Reference frame for the six degrees-of-freedom of ship motion. Positive rotations about each axis are counterclockwise.

The recorded ship motion data to be replicated by the robotic simulator is in the body-fixed coordinate frame. For the scope of this work, the vessel is assumed to be operating in the straight-ahead condition, i.e. the zero-yaw design condition, where the ship bearing is ignored as this condition is common for tows, lifts and launch and recovery [10]. It is therefore specified that the ship motion simulator must produce motion in 5 degrees-of-freedom: the translations surge, sway and heave, and the roll 
and pitch rotations depicted in Figure 2.2. While the ship motion will be reproduced in the body-fixed frame, ocean waves will be modelled in the world frame, which will be defined later in this thesis.

\subsection{Modelling Ocean Waves}

The six degrees-of-freedom motion of the host vessel can be resolved from the measurements of an onboard Inertial Measurement Unit (IMU). However, the surface wave motion cannot be deduced from the measured ship motion due to the dampening effect that the vessel enacts upon the surface wave motion. As the surface waves are a primary consideration of this thesis, it is important that they can be modelled and reproduced experimentally. The waves that can be achieved in the narrow channel of the flume tank will be unidirectional, shallow water waves that are propagated horizontally by the flume tank flow speed. It is desired that the wavemaker design can reproduce both regular and irregular waves. A regular wave is monochromatic, having a single frequency and amplitude. Irregular waves are random and representative of real ocean waves. The theory of both types of waves are introduced in the following sub sections.

\subsubsection{Regular Waves}

Small-amplitude water wave theory produces the profile of a monochromatic ocean wave under the simplifying assumptions that the fluid motion is irrotational and the fluid is incompressible [11]. Under the assumptions of irrotational and incompressible flow, there exists a velocity potential that satisfies the continuity equation

$$
\nabla \cdot \nabla \phi=0
$$




\subsection{MODELLING OCEAN WAVES}

where $\phi$ is the velocity potential, a scalar quantity whose gradient $\nabla$ equals the velocity field. Equation 2.1 is the Laplace equation: the governing second-order differential equation which describes the conservation of mass [9]. Hence, solving for the water wave profile becomes a boundary value problem.

The two-dimensional boundary value problem for regular water waves is illustrated in Figure 2.3 where the main boundary conditions are identified: the parameter $L$ represents the wavelength, $H$ is the wave height and $h$ is the water depth from still water level, $\eta(x, t)$ represents the position of the free surface at position $x$ and at time $t$ relative to the plane $z=0$ and by definition has a zero spatial and temporal mean. The Bottom Boundary Condition (BBC) describes the condition of the lower boundary of Figure 2.3. For a horizontal bottom, such as the case of the flume tank at Carleton University, the no flow condition applies, where

$$
-\frac{\partial \phi}{\partial z}=0 \quad \text { on } z=-h
$$

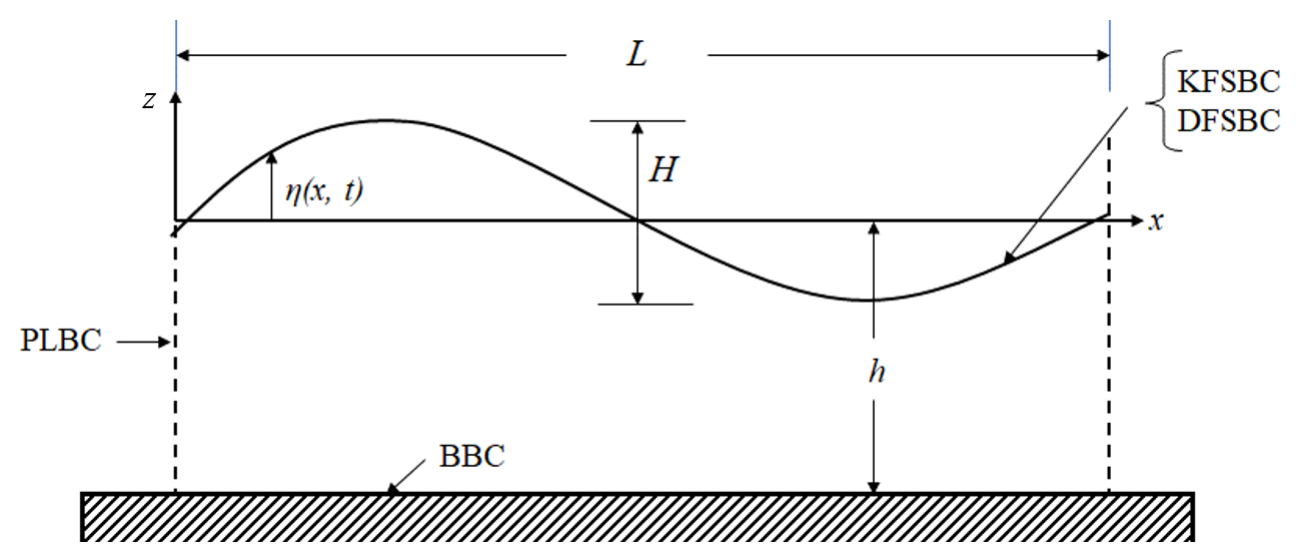

Figure 2.3: Schematic of the boundary value problem for a two-dimensional water wave.

Two boundary conditions exist at the free surface: the Kinematic Free Surface Boundary Condition (KFSBC) and the Dynamic Free Surface Boundary Condition (DFSBC). The KFSBC ensures that there is no flow across the free surface at 
the water-to-air interface such that

$$
-\frac{\partial \phi}{\partial z}=\frac{\partial \eta}{\partial t}-\frac{\partial \phi}{\partial x} \frac{\partial \eta}{\partial x} \quad \text { on } z=\eta(x, t)
$$

and the DFSBC describes the requirement for uniform pressure distribution across the free surface boundary, where

$$
-\frac{\partial \phi}{\partial t}+\frac{1}{2}\left[\left(\frac{\partial \phi}{\partial x}\right)^{2}+\left(\frac{\partial \phi}{\partial z}\right)^{2}\right]+g \eta=G(t) \quad \text { on } z=\eta(x, t)
$$

with $g$ as the acceleration due to gravity and $G(t)$ as the uniform distribution.

The final boundary conditions applied to the water wave problem are the Periodic Lateral Boundary Conditions (PLBC). These lateral boundary conditions are appropriate for channel flow and constrain the flow exiting the downstream lateral boundary to re-enter the upstream lateral boundary. The periodicity conditions for both time and space are

$$
\begin{aligned}
\phi(x, t) & =\phi(x+L, t) \\
\phi(x, t) & =\phi(x, t+T) .
\end{aligned}
$$

The linearized solution to the water wave boundary value problem, was proposed by Dean and Dalrymple [9], and the velocity potential $\phi$ is,

$$
\phi=\frac{H}{2} \frac{\cosh k(h+z)}{\omega \cosh k h} \cos k x \sin \omega t
$$

where the wave number $k=2 \pi / L$ is related to the wave frequency $\omega=2 \pi / T$ by the dispersion relationship,

$$
\omega^{2}=g k \tanh k h
$$

Retaining the linear terms of the DFSBC in Equation 2.4, the position of the free 


\subsection{MODELLING OCEAN WAVES}

surface $\eta$ is

$$
\eta(x, t)=\frac{1}{g} \frac{\partial \phi}{\partial t}=\frac{H}{2} \cos k x \cos \omega t
$$

The wave form of the solution in Equation 2.6 is a standing wave, a wave form that often occurs when an incident wave is completely reflected by vertical walls and appears to be stationary. The linear solution in Equation 2.6 is extended to progressive waves that move through the medium, whereby two standing waves $90^{\circ}$ out of phase are superimposed [9]. The velocity potential for a progressive wave is then

$$
\phi=-\frac{H}{2} \frac{g}{\omega} \frac{\cosh k(h+z)}{\cosh k h} \sin (k x-\omega t)
$$

with water surface elevation given as

$$
\eta(x, t)=\frac{1}{g} \frac{\partial \phi}{\partial t}=\frac{H}{2} \cos (k x-\omega t)
$$

The dispersion relationship in Equation 2.7 describes the dispersion of a propagating wave field that occurs due to the different wave speeds of the various frequency components. Progressive waves propagate at wave speed $C=L / T$ and the dispersion equation quantifies the manner in which progressive waves slow down as they enter shallow water, expressed as

$$
C=\frac{L_{o}}{T} \tanh k h
$$

where $L_{o}$ is the wave length in deep water, i.e where $h / L_{o}>0.5$ [9]. As the water depth in the flume tank has a maximum depth of $86 \mathrm{~cm}$ (34 in), the wave speed of generated waves in the tank will be described by Equation 2.11.

Waves may propagate via various modes and one mode of interest to this work is wave propagation via the fluid current. In the scope of this work, waves will be propagating at the flume scale flow velocity of $0.3 \mathrm{~m} / \mathrm{s}$, which will represent the 
the mean ship velocity in towed body operations. Assuming the current $U_{o}$ is flowing in the direction of propagating waves, one solution to the boundary value problem proposed by Dean and Dalrymple is

$$
\phi=-U_{o} x+\frac{g H}{2 \omega\left(1-U_{o} / C\right) \cosh k h} \cosh k(h+z) \cos (k x-\omega t)
$$

The wavemaker must be capable of producing waves propagated by a current, the theory of which will be explored in greater detail in Chapter 5 .

\subsubsection{Irregular Waves}

The sea surface is composed of many waves moving in different directions with different frequencies, amplitudes and phases. Adequately describing the sea surface requires a large number of superimposed, monochromatic waves [9]. Random ocean waves are described by an energy density spectrum $S(\omega)$, which describes the energy content of an ocean wave and its distribution over a range of frequencies [12]. Many empirical spectrum formulas exist to define ocean waves. Each formula is site-specific, but the most common spectral models are the JONSWAP, Pierson-Moskowitz (P-M) and the Bretschneider models. The P-M spectrum is a one parameter spectrum representing fully developed seas generated by local winds. A fully developed sea is a sea condition produced by winds blowing steadily over a large distance for several days where the waves are in equilibrium with the wind [13]. The two-parameter Bretschneider spectrum is the most commonly used model for non-directional spectra in the open ocean and has widely replaced the P-M spectrum as it does not require the sea to be fully developed [14]. The JONSWAP spectrum is often used to describe the fetch-limited conditions in the North Sea [15]. A fetch-limited sea is condition where wave height is limited by the size of the wave generation area, or fetch [16]. It is a special case of the Bretschneider spectrum to which a peak enhancement factor accounting for fetch-limited conditions is added [14]. For the scope of this work, the Bretschneider will be used for developing irregular wave profiles to generate waves 


\subsection{MODELLING OCEAN WAVES}

in the wave tank due to its widespread applicability to many ocean regions and the simplicity of its empirical definition.

The Bretschneider spectrum model is empirically defined as

$$
S(\omega)=\frac{1.25}{4} \frac{\omega_{o}^{4}}{\omega^{5}} H_{s} e^{-1.25\left[\frac{\omega}{\omega_{o}}\right]^{-4}}
$$

where $\omega$ is the angular frequency, $w_{o}$ is the peak frequency of the spectrum and $H_{s}$ is the significant wave height. The peak frequency is related to the significant height of the wave $H_{s}$, a statistical parameter that describes the mean wave height of the highest third of the waves in the spectrum. The relationship between $\omega_{o}$ and $H_{s}$ is $[13]$

$$
\omega_{o}=0.4 \sqrt{\frac{g}{H_{s}}}
$$

which describes how the peak frequency shifts with the value of the significant wave height. Figure 2.4 plots the Bretschneider spectrum, $S(\omega)$, as a function of wave frequency $\omega$ for various significant wave heights, $H_{s}$ which are represented by various line styles.

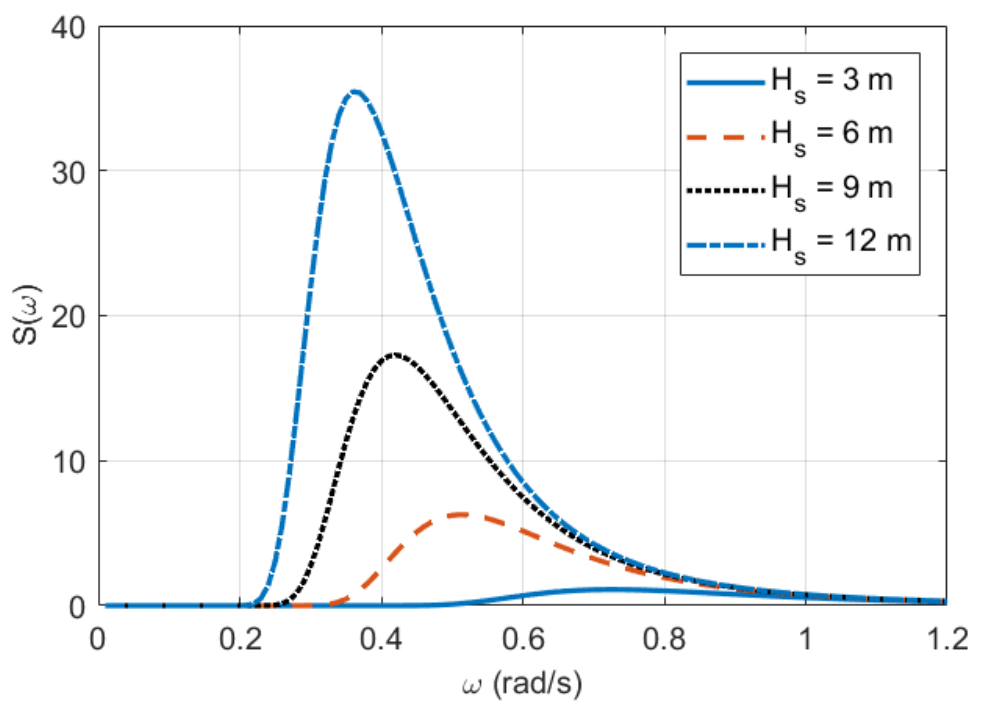

Figure 2.4: Bretschneider wave energy spectra.

Simulating irregular wave motion requires the time history of the wave to 
be computed from the wave spectrum model [12]. It is often practical to first express the wave spectrum in terms of frequency $f$ instead of angular frequency $\omega$. The Bretschneider spectrum then becomes [13]

$$
S(f)=2 \pi \frac{1.25}{4} \frac{f_{o}^{4}}{2 \pi f^{5}} H_{s} e^{-1.25\left[\frac{f}{f_{o}}\right]^{-4}}
$$

where $f_{o}$ is the peak frequency in Hz. The entire spectrum in Equation 2.15 is divided into $N$ frequency bands of width $\Delta f$ where the wave height within the frequency band $n$ is derived as [12],[17]

$$
H\left(f_{n}\right)=2 \sqrt{2 S\left(f_{n}\right) \Delta f}
$$

In Equation 2.16, $f_{n}$ is the mean frequency within the $n$th frequency band. The wave profile in the time-domain is obtained from

$$
\eta(x, t)=\sum_{n=1}^{N} \frac{H(n)}{2} \cos [k(n) x-2 \pi f(n) t+\epsilon(n)]
$$

where the wave number $k$ is computed from dispersion relationship in Equation 2.7, and $\epsilon$ is a random phase assigned to each frequency band to maintain the randomness of the wave profile [12].

The significant wave height and modal period parameters that define the Bretschneider spectrum also define a common term "sea state".

\subsubsection{Sea States}

Sea states describe the general condition of the free surface of the ocean at any given time and location. Several statistical parameters are used to describe sea states, such as wave height and period. While there are many sea state definition standards, this thesis will follow the definitions set out by the North Atlantic Treaty Organization (NATO) and the World Meteorological Organization (WMO). The NATO/WMO sea state code defines sea states by significant wave height and modal period, where the 


\subsection{MODELLING OCEAN WAVES}

modal period corresponds to the peak frequency of the wave energy spectrum. For the purposes of this thesis, it is desired that sea states 3 to 6 are replicated in the flume tank. Table 2.1 summarizes sea states 1 to 6 [18]. The highlighted cells are the sea states that will be recreated in the water channel.

\begin{tabular}{r|cc|ccc}
\multirow{2}{*}{ Sea State } & \multicolumn{2}{|c|}{ Significant Wave Height $[\mathbf{m}]$} & \multicolumn{2}{c}{ Modal Period $[\mathrm{s}]$} \\
\cline { 2 - 5 } & Range & Mean & Range & Most Probable \\
\hline $\mathbf{1}$ & $0-0.1$ & 0.05 & - & - \\
$\mathbf{2}$ & $0.1-0.5$ & 0.3 & $3.3-12.8$ & 7.5 \\
$\mathbf{3}$ & $0.5-1.25$ & 0.88 & $5.0-14.8$ & 7.5 \\
$\mathbf{4}$ & $1.25-2.5$ & 1.88 & $6.1-15.2$ & 8.8 \\
$\mathbf{5}$ & $2.5-4.0$ & 3.75 & $8.3-15.5$ & 9.7 \\
$\mathbf{6}$ & $4.0-6.0$ & 5.0 & $9.8-16.2$ & 12.4
\end{tabular}

Table 2.1: Sea state definition by NATO STANAG 4194 Annex D.

Visually, the sea states can be plotted by the relation between the modal period and significant wave height. For the Bretschneider spectrum described in the section above, this relationship is fixed and the modal period $T_{p}$ is, [12].

$$
T_{p}=\sqrt{240 \frac{H_{s}}{g}} .
$$

Figure 2.5 plots the modal period $T_{p}$ as a function of significant wave height $H_{s}$ where the wave height and period ranges from Table 2.1 are shown. In Figure 2.5 the Bretschneider spectrum line passes through the sea states depicts where the the Bretschneider spectrum model is capable of describing each sea state.

To recreate sea states 3 through 6 in the flume tank, the parameters must be properly scaled. To accomplish the task of scaling the full scale ocean parameters to the flume scale, dimensional analysis is performed using Froude scaling. Froude scaling was selected due to its applicability to free-surface flows that are governed by gravitational forces. For the Froude criterion to be satisfied, the following relationship must be met

$$
\frac{\Pi_{v}^{2}}{\Pi_{g} \Pi_{l}}=1,
$$

where where $\Pi$ indicates a ratio of the full scale parameters to the flume-scale pa- 
CHAPTER 2. BACKGROUND AND LITERATURE REVIEW

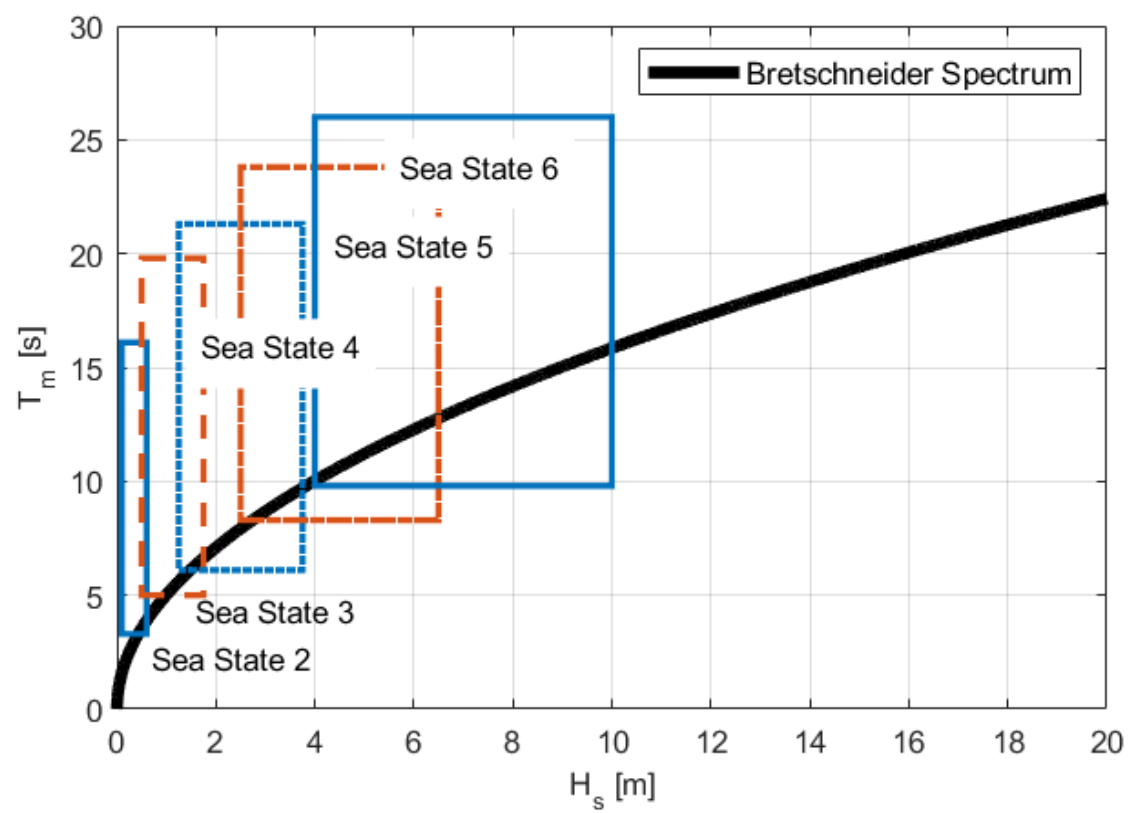

Figure 2.5: Bretschneider spectrum with sea states.

rameters, $\Pi_{v}$ is the velocity ratio, $\Pi_{g}$ is the ratio of gravitational fields, and $\Pi_{l}$ is the ratio of characteristic length, where the characteristic length represents both the significant wave height and wavelength. The modal period $T_{p}$ is related to wavelength $L$ and mean ship velocity $U_{o}$ by

$$
T_{p}=\frac{L}{U_{0}} .
$$

Full-scale mean ship velocity is taken from the DSTO report [19] as 3.66 $\mathrm{m} / \mathrm{s}$. The surface flume tank flow speed of $0.305 \mathrm{~m} / \mathrm{s}$ is used as an approximation of flume-scale flow speed, as the waves are reasonably far from the bottom of the tank where the flow speed is lower [1]. Full-scale wavelength is calculated from the modal period ranges in Table 2.1 and both the full-scale and flume-scale gravitational accelerations are taken to be $9.81 \mathrm{~m} / \mathrm{s}$. Using the Froude scaling criterion in Equation 2.19, the scaled sea states 3 to 6 are summarized in Table 2.2.

It is the sea states summarized in Table 2.2 that must be replicated by a wave generator. The third objective of this work requires that a conceptual design with all associated theory of wavemaker design be provided. An overview of existing 


\subsection{WAVE GENERATORS}

\begin{tabular}{r|ccccc}
\multirow{2}{*}{ Sea State } & \multicolumn{2}{|c|}{ Significant Wave Height $[\mathrm{m}]$} & \multicolumn{2}{c}{ Modal Period $[\mathrm{s}]$} \\
\cline { 2 - 5 } & Range & Mean & Range & Most Probable \\
\hline $\mathbf{3}$ & $0.004-0.009$ & 0.007 & $0.42-1.23$ & 0.63 \\
$\mathbf{4}$ & $0.009-0.017$ & 0.013 & $0.51-1.27$ & 0.73 \\
$\mathbf{5}$ & $0.017-0.028$ & 0.023 & $0.69-1.29$ & 0.81 \\
$\mathbf{6}$ & $0.028-0.042$ & 0.035 & $0.83-1.35$ & 1.03
\end{tabular}

Table 2.2: Flume-scale sea state definition based on Froude scaling.

wave generator designs is presented in the next section.

\subsection{Wave Generators}

In this section, an overview of the common types of wave generators is presented, where the advantages and disadvantages of each type of generator are provided in order to select a suitable option for the flume tank. A comprehensive review of the different types of wavemakers is provided by Biesel and Suquet [20] and Chappell [21]. The wave generator types are evaluated for use at Carleton based on the ease of computing the required wavemaker displacement, i.e. stroke length, from the desired wave amplitude, and the compatibility of the wave generator for generating waves in a short channel. The wavemaker design must also allow for flow across the lateral boundaries of the channel to simulate ocean current. Priority is to be placed on the wavemaker type with fewer submerged parts as submerged parts are more difficult to remove and maintain. The three common wave generator designs are pistontype, flap-type and plunger-type wave generators. The following sub sections briefly describe the wavemakers, while Table 2.3 provides a summary of the options with the evaluation criteria. 


\section{CHAPTER 2. BACKGROUND AND LITERATURE REVIEW}

\subsubsection{Piston-type Wavemakers}

The piston-type wavemaker, depicted in Figure 2.6, consists of a moveable wall that pushes water in the longitudinal $x$ direction as it is moved back and forth in the water channel. This type of wavemaker is best suited for generating shallow water waves, and therefore a long channel is needed to permit wave stabilization when deep water waves are desired. An analytic solution exists for calculating the amplitude [9],[20]; however, this type of wavemaker is actuated inside the water channel and requires substantial mechanical components to be submerged. The piston type wavemaker typically does not allow flow through the channel, where no water exists behind the piston. A wavemaker that has fluid on only one side of the moving component is called a dry back design [22]. From the above analysis it is judged that a piston style system is not well suited for the flume tank at Carleton.

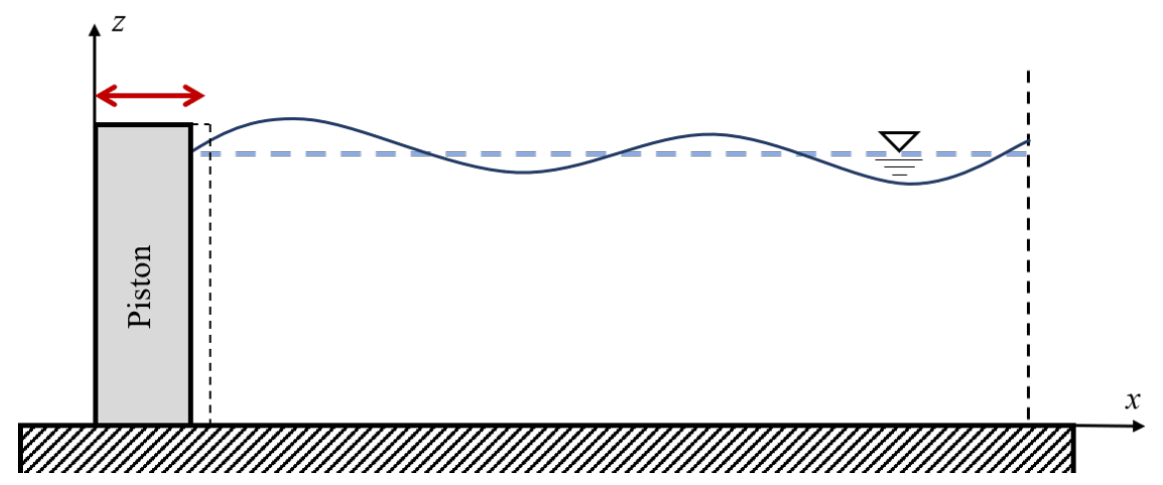

Figure 2.6: Schematic of a laboratory scale piston-type wavemaker.

\subsubsection{Flap-type Wavemakers}

Flap-type wavemakers include both flexible and rigid flap types, illustrated in Figure 2.7. Rigid type flaps, schematic a) in Figure 2.7, are typically of simple construction that are most suited for generating deep water waves. An analytic solution exists for calculating the wave amplitude and required actuator amplitude [9],[20]. The flexible flap type, schematic b) in Figure 2.7, can closely approximate water particle 


\subsection{WAVE GENERATORS}

motion along the height of the wave channel and can produce stable and accurate motions directly in front of the flap. The latter is advantageous for shorter length water channels. The amplitude of the waves generated is not easily calculable for actuation purposes [20].

Although flap-type wavemakers may be actuated above water, as with the piston-type wavemaker, flap-type wavemakers require some mechanical components to be submerged, most notably the hinge. Furthermore, like the piston type wavemaker, flap type systems are also typically implemented as dry back systems. For the reasons stated above, a flap style system is not well suited for the flume tank at Carleton.

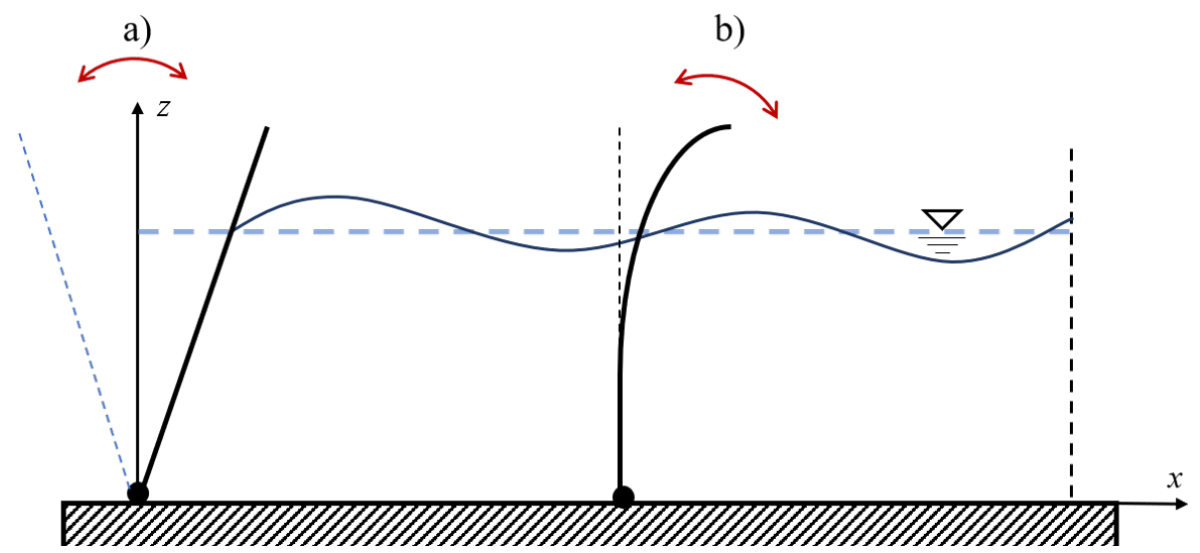

Figure 2.7: Schematic of a laboratory scale a) rigid flap and b) flexible flap type wavemaker.

\subsubsection{Plunger-type Wavemakers}

Plunger-type wavemakers are the less commonly used than either piston or flap-type wavemakers. Figure 2.8 illustrates the plunger-type wavemaker in a water channel. Waves are produced by the displacement of the plunger being periodically thrust into and withdrawn from the water, where wave length and height are controlled by varying the period of oscillation and plunger displacement. The plungers may be various shapes and cross-sections but the wedge shaped plunger is most common. While an analytical solution does not exist to solve for wave amplitude, several numerical 
solutions exist like those formulated by Wang [23] and Wu [24]. The main disadvantage of plunger-type wavemakers is that they require longer channels to achieve wave stabilization; however, as it will be shown in Section 5.1.1, it is theoretically possible to achieve stable waves with the available test section length in the flume tank. Plunger-type wavemakers do allow for flow across the lateral boundaries as the plunger is cyclically retraced throughout operation.

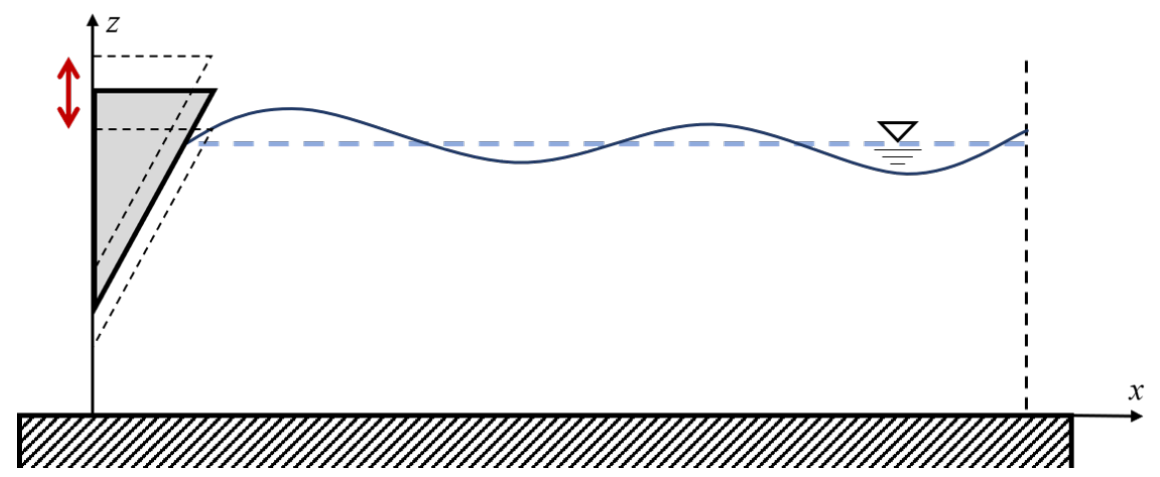

Figure 2.8: Schematic of a laboratory scale plunger-type wavemaker.

Table 2.3 provides a summary of the existing wavemaker types and evaluates them based on the ease of computing the required wavemaker displacement, the compatibility of the design with the water channel at Carleton, the amount of submerged components and the design's ability to allow for flow across the boundaries.

\begin{tabular}{|l|l|l|l|l|}
\cline { 2 - 5 } \multicolumn{1}{c|}{} & $\begin{array}{l}\text { Analytical } \\
\text { Solution }\end{array}$ & $\begin{array}{l}\text { Short } \\
\text { Channel }\end{array}$ & $\begin{array}{l}\text { Submerged } \\
\text { Components }\end{array}$ & $\begin{array}{l}\text { Boundary } \\
\text { Flow }\end{array}$ \\
\hline Piston & $\begin{array}{l}\text { Known } \\
{[9,20]}\end{array}$ & Not well suited & Fully (poor) & No \\
\hline Flap & $\begin{array}{l}\text { Known } \\
{[9,20]}\end{array}$ & Well suited & Fully (poor) & No \\
\hline Plunger & $\begin{array}{l}\text { Numerical only } \\
{[23,24]}\end{array}$ & Possible & $\begin{array}{l}\text { Can be } \\
\text { retracted }\end{array}$ & Yes \\
\hline
\end{tabular}

Table 2.3: Summary of existing wavemaker designs based on design criteria.

Based on the overview of wave generator types above, the plunger-type wavemaker meets most of the design requirements. The plunger-type wavemaker can be mounted completely above the tank to avoid submerged components, and it is 


\subsection{WAVE SYNCHRONIZATION}

possible to compute the required stroke length to reproduce a given wave amplitude. Plunger-type wavemakers are also versatile as the wavemaker can be outfitted with different plunger shapes and sizes, depending on the wave profiles to be replicated.

Now that it has been established that waves could be replicated via a plunger-type wave generator, to fulfill the first objective of this thesis, wave synchronization methods need to be examined for launch and recovery operations.

\subsection{Wave Synchronization}

Wave synchronization is a concept that refers to the act of inducing an object to follow the motion of waves. A simple approach to synchronizing the motion of a towed body with the wave motion in an overside crane operation could be to allow the boom of the crane to "nod" up and down with the wave motion. However, at full scale, this method could require large on-deck equipment to operate unpredictably with the changing wave conditions. As a primary motivation for this work is to advance efforts of improving safety, this research will focus only on investigating winch based control methods to achieve wave synchronization.

In the work presented by Sagutun et al. [2], combined heave compensation and wave synchronization compensator strategies are investigated for heavy-lift offshore crane operations. The one dimensional equation of motion for a submerged payload in a lifting operation, as presented in Sagutun et al. [2], draws from resources, such as Faltinsen [11], Newman [25] and standards set by Det Norske Veritas (DNV) [26], and previous work by Greenhow and Yanbao [27], that collectively present the formulation of the hydrodynamics of submerged and partially submerged bodies in both lifting and lowering operations.

Figure 2.9 shows the schematic of the laboratory scale moonpool adapted

from Sagutun et al. [2] and Johansen et al. [5] that consists of an electric motor and 
spherical payload attached to a wire that runs over a pulley that is suspended from a spring. A spring was used to simulate wire elasticity in the scaled model [2]. The vertical positions of the vessel, payload, motor and pulley are represented by $z_{0}, z_{T B}$, $z_{m}$ and $z_{s}$ respectively. $z_{r}$ is the vertical position of the payload relative to the wave surface elevation $\eta$ at the centre of the moonpool. As defined by Sagutun et al. [2], all coordinates are positive downwards where coordinates $z_{T B}, z_{m}$ and $z_{s}$ are in the body-fixed vessel frame and coordinates $z_{r}$ and $\eta$ are defined with respect to the still water level in the world frame.

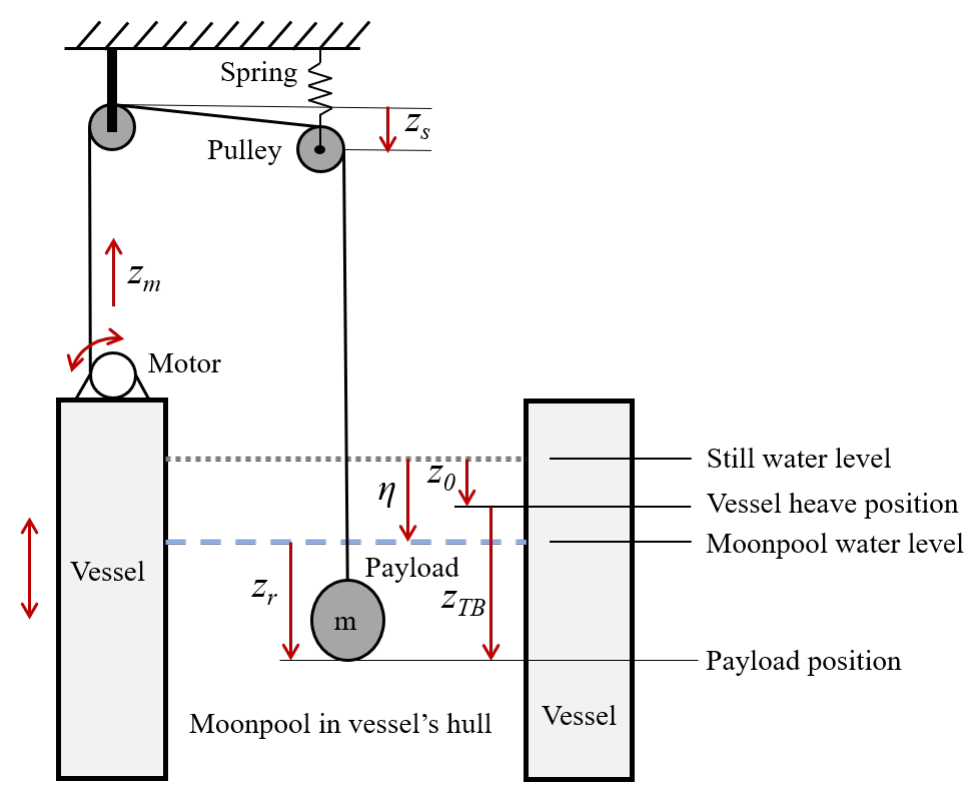

Figure 2.9: Schematic of a laboratory scale moonpool with definition of the coordinate system.

Saguten et al. reduced the equation of motion of the payload to one dimension to consider only vertical motion of the payload [2]. This simplification was made by assuming that the vessel is kept at a mean fixed position and heading, relative to incoming waves, and neglecting roll and pitch motion of the vessel, which are assumed to be small compared to heave motion in the moonpool environment. The equations of motion of the motor and payload are defined as,

$$
m_{m} \ddot{z}_{m}=F_{m}+F_{T}
$$




\subsection{WAVE SYNCHRONIZATION}

$$
m\left(\ddot{z}_{T B}+\ddot{z}_{0}\right)=m g+f_{z}-F_{T}
$$

where $z_{m}=R \theta_{m}, m_{m}=J_{m} / R^{2}$ and $F_{m}=T_{m} / R$ with the pulley on the motor shaft having radius $R$, while $\theta_{m}, J_{m}$ and $T_{m}$ are the motor angle, motor inertia and motor torque, respectively. The forces $F_{T}$ and $f_{z}$ are the wire tension and hydrodynamic and static force on the payload of mass $m$. For the pulley with mass $m_{s}$ suspended by the spring, the equation of motion is,

$$
m_{s} \ddot{z}_{s}+d_{s} \dot{z}_{s}+k_{s} z_{s}=F_{T}
$$

where $d_{s}$ and $k_{s}$ are the damping and spring coefficients. As payload motion is due to the wire being reeled in and payed out as well as wire elongation, in the body-fixed vessel frame $z_{T B}=z_{m}+z_{s}$. With the position of the payload now defined in terms of motor and pulley positions, Equation 2.23 is rearranged to solve for the line tension $F_{T}$,

$$
F_{T}=m_{s}\left(\ddot{z}_{T B}-\ddot{z}_{m}\right)+d_{s}\left(\dot{z}_{T B}-\dot{z}_{m}\right)+k_{s}\left(z_{T B}-z_{m}\right) .
$$

Sagutun et al. [2], Johansen et al., [5] and Fossen et al., [28] formulated the hydrodynamic loads on the payload in the vertical direction given by,

$$
f_{z}=-\rho g V\left(z_{r}\right)-\rho V\left(z_{r}\right) \ddot{z}-Z_{\ddot{z}_{r}}\left(z_{r}\right) \ddot{z}_{r}-\frac{\partial Z_{\ddot{z}_{r}}\left(z_{r}\right)}{\partial z_{r}} \dot{z}_{r}^{2}-\frac{1}{2} \rho C_{D} A_{p z} \dot{z}_{r}\left|\dot{z}_{r}\right|-d_{l} \dot{z}_{r},
$$

where $\rho$ is the density of water, $g$ is the acceleration due to gravity, $\forall$ is the volume of water displaced by the payload, $Z_{\ddot{z}_{r}}$ is the position dependent added mass from [26] and [27], which accounts for the portion of the fluid surrounding the payload that increases the inertia of the payload, $C_{D}$ is the drag coefficient, $A_{p z}$ is the projected effective drag area in the vertical direction and $d_{l}$ is linear drag. The first term of Equation 2.25 is the buoyancy force, the second term represents the FroudeKriloff pressure force [26] and the third represents the contribution of added mass. The hydrodynamic slamming loads that occur at the water exit and entry zone are represented by $-\frac{\partial Z_{\ddot{z}_{r}}\left(z_{r}\right)}{\partial z_{r}} \dot{z}_{r}^{2}$, where the negative sign means that slamming forces are 


\section{CHAPTER 2. BACKGROUND AND LITERATURE REVIEW}

directed upwards as the payload hits the water during entry. Finally the last two terms of Equation 2.25 represent the viscous and linear drag.

For motion compensation of both heave and wave motion, Sagutun et al. [2] employed feedforward compensator strategies as the main disturbances could be reliably estimated from experimental measurements. Compensator performance was measured by wire tension and hydrodynamic loads on the payload, where it was desired that the peak values and variance of both performance metrics be minimized. For the active heave compensation feedforward strategy, it was desired to make the payload track a given trajectory toward the water surface by decoupling the payload motion from the vessel heave motion. From Johansen et al. [5], the payload reference signal $\dot{z}_{d}$ is obtained by adding an estimate of the vessel's vertical velocity $\dot{\hat{z}}_{0}$ to the motor speed reference signal $\dot{z}_{m}^{*}$ and expressed as

$$
\dot{z}_{d}=\dot{z}_{m}^{*}+\dot{\hat{z}}_{0}
$$

where the estimated vertical velocity of the vessel $\dot{\hat{z}}_{0}$ is obtained by integrating an accelerometer signal in the body-fixed vessel frame.

For wave synchronization, the objective of the feedforward compensator is to minimize variations in the hydrodynamic loads on the payload during the water entry phase by synchronizing the payload motion with the moonpool water motion during water entry and exit. From the equation of motion of the payload, Equation 2.22, and Equation 2.25, Johansen et al. [5] show that minimal tension variations are achieved by minimizing the relative vertical velocity between the payload and water surface $\dot{z}_{r}$. Defining $\dot{\eta}_{0}$ as the velocity of the wave surface in the body-fixed vessel frame, it follows that $\dot{z}_{r}=\dot{z}_{T B}-\dot{\eta}_{0}$. As wave amplitude decays with depth of the payload $z_{T B}$, Sagutun et al. [2] defined a piece-wise function $\kappa\left(z_{T B}\right)$ to scale the wave amplitude accordingly such that $\dot{z}_{r}=\dot{z}_{T B}-\dot{\eta}_{0} \kappa\left(z_{T B}\right)$. The wave synchronization 


\subsection{WAVE SYNCHRONIZATION}

feedforward compensator is then

$$
\dot{z}_{d}=\dot{z}_{m}^{*}+\dot{\hat{\eta}}_{0} \kappa\left(z_{T B}\right)
$$

where $\dot{\hat{\eta}}_{0}$ is the estimate of the velocity of the wave surface elevation. Consistent with Sagutun et al. [2], one can assume the water's vertical velocity is constant from the water surface to the bottom of the moonpool at the vertical position $h_{m}$, and that wave amplitude decays exponentially with depth $z_{T B}$ thereafter; hence $\kappa\left(z_{T B}\right)$ is defined as

$$
\kappa\left(z_{T B}\right)=\left\{\begin{array}{ll}
1 & z_{T B} \leq h_{m} \\
e^{-k\left(z_{T B}-h_{m}\right)} & z_{T B}>h_{m}
\end{array} .\right.
$$

Finally, Sagutun et al. [2] define a factor $\alpha\left(z_{T B}\right)$ to blend the active heave compensation with wave synchronization such that wave synchronization only occurs during the breach phase where the blended compensator strategy is given by

$$
\dot{z}_{d}=\dot{z}_{m}^{*}+\dot{\hat{\eta}}_{0} \alpha\left(z_{T B}\right) \kappa\left(z_{T B}\right)+\dot{\hat{z}}_{0}\left(1-\alpha\left(z_{T B}\right) \kappa\left(z_{T B}\right)\right)
$$

where $\alpha(z)$ is a position dependent factor defined such that it changes from zero to one smoothly over the height of the payload $h_{p}$ as the payload is being submerged,

$$
\alpha\left(z_{T B}\right)=\left\{\begin{array}{ll}
0 & z_{T B}<0 \\
z_{T B} / h_{p} & 0 \leq z_{T B} \leq h_{p} \\
1 & z_{T B}>h_{p}
\end{array} .\right.
$$

Sagutun et al. [2] also investigated the compensator control strategies that were validated against laboratory scale experimental results. Tests were run in different scaled sea states for both regular and irregular waves. It was observed that wave synchronization by itself or in combination with heave compensation significantly reduced the variability in wire tension with "good repeatability" for the regular wave 


\section{CHAPTER 2. BACKGROUND AND LITERATURE REVIEW}

tests. The largest reduction was found when both wave synchronization and heave combination were used, where the variability of line tension was reduced up to $54 \%$.

Eikeland [7] advanced the wave synchronization crane control developed by Sagutun et al. [2] to investigate wave synchronization for overside cranes where the payload is being lifted from the sea. The same heave compensation and wave synchronization control strategies described above were employed as control strategies to obtain the control inputs for an A-frame crane. Eikeland [7] followed the same assumptions as in [2] to simplify the formulation to the one-dimensional case where the vessel was assumed to be kept at a mean fixed position and roll and pitch motion were ignored. The model was simulated in Simulink where the results suggested that a blended heave compensation and wave synchronization strategy provided better tension variability.

Eikeland's simplifications for the one-dimensional case are not realistic for many over-side crane lifting operations. For many launch and recovery operations the vessel is often moving at a constant speed such that drag forces on the payload can no longer be assumed to only be in the vertical direction. Additionally, vessel roll and pitch motion cannot be ignored like in the moonpool environment. Moreover, cable dynamics should not be ignored. This thesis seeks to improve upon these shortcomings in order to more accurately model lifting operations of an over-side crane operation.

\subsection{Summary}

In this chapter, various topics were discussed that are relevant to the design of a robotic ship motion simulator and wave generator. Ocean wave theory was investigated in depth, where both linear wave theory and the modelling of irregular waves were discussed. The Bretschneider spectrum will be used to model irregular waves

due to its direct applicability to the definition of sea states. A plunger-type wave maker will be designed for generating the wave profiles experimentally. Compensator 


\subsection{SUMMARY}

strategies were also investigates for lifting operations where it was found to be advantageous to combine heave compensation and wave synchronization when lifting through the wave zone.

The following chapter describes the three-dimensional formulation of the wave synchronization control strategy for lifting operations. 


\section{Chapter 3}

\section{Wave Synchronization Model and Simulation}

This chapter describes the advancement of a computer simulator that is used to model a flume-scale towed body system in a lifting operation. Waves are introduced to the model such that wave synchronization can be explored. The simulator is based on the work of Calnan et al. [1, 29, 30] and consists of a cable model and towed system developed in MATLAB and Simulink.

Section 3.1 provides an overview of the computer simulator developed by Calnan et al. where the theory behind the rigid body cable model is discussed. Section 3.2 introduces the formulation of regular and irregular waves and the ship motion used during simulations. The hydrodynamic loads on the cable and towed body are discussed in Section 3.3 for when the body is fully submerged and during breach. Section 3.4 discusses the combined heave compensation and wave synchronization strategies based on the various setpoint algorithms [29]. The simulation results are presented and interpreted in Section 3.5 while Section 3.6 summarizes the results. 


\subsection{TOWED BODY SIMULATOR OVERVIEW}

\subsection{Towed body simulator overview}

Calnan et al.'s [29] three dimensional towed body simulator consists of models for a winch, cable and towed body subsystems. The simulator was developed to validate the flume scale experimental results that quantified the performance of the AHC setpoint algorithms. The experimental setup consisted of a monofilament nylon cable attached to a towed sphere. The system did not include a sheave as the cable went directly into the water. Figure 3.1 shows the system schematic that was developed in the Simulink environment based on the flume scale experimental system. The waterline is located $46 \mathrm{~cm}$ below the winch at its start position.

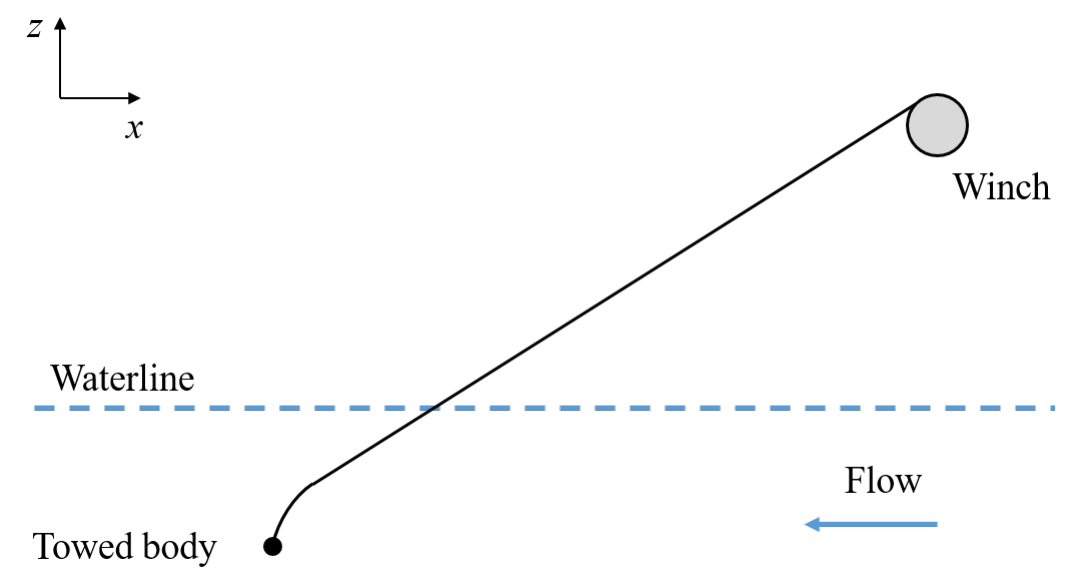

Figure 3.1: Schematic of the towed body simulator system. The $y$-axis is directed into the page.

Calnan et al.'s cable model is composed of a series of rigid body linkages $L_{i}$, represented by cylindrical bodies, connected by a universal joint [29]. The universal joint between the linkages allows rotation about the $x_{i}$ and $y_{i}$ axes of each linkage, but restricts $z_{i}$ rotation along the cable, consistent with the assumption of minimal tow cable torsion described by Hover et al. [31], Driscoll et al. [32] and Kamman and Huston [33]. Figure 3.2 depicts the typical configuration of the cable linkages $L_{i}$ and $L_{i+1}$ connected by a universal joint with the body-fixed coordinate frame located at the centre of gravity of each cable link. The $z$-axis is always aligned with the longitudinal axis of the cable linkage while the $x$ and $y$ axes are normal to the cable 


\section{CHAPTER 3. WAVE SYNCHRONIZATION MODEL AND SIMULATION}

linkage. Rotational stiffness and rotational damping are applied at each universal joint. External loads on the cable and towed body are applied at the centre of gravity of each body. Table 3.1 lists the parameters used in Calnan et al.'s simulator [29] that will also be used in the current thesis work.

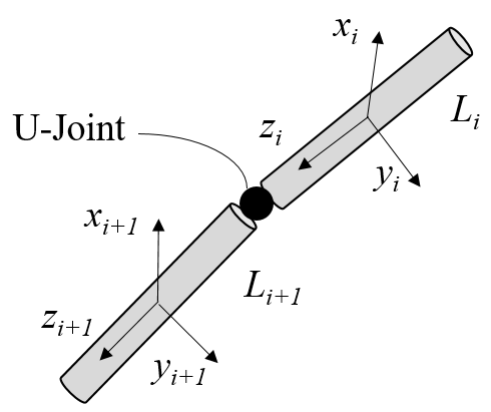

Figure 3.2: Configuration of tow cable linkages connected by a universal joint with respective co-ordinate frames. Adapted from [1].

\begin{tabular}{|c|c|c|}
\hline Subsystem & Simulator Parameter & Value \\
\hline \multirow{4}{*}{ Cable } & Diameter & \multirow{9}{*}{$\begin{array}{l}0.45 \mathrm{~mm} \\
0.20 \mathrm{~g} / \mathrm{m} \\
4.488 \times 10^{-6} \mathrm{Nm} / \mathrm{deg} \\
3.116 \times 10^{-9} \mathrm{Nms} / \mathrm{deg} \\
10 \mathrm{~mm} \\
1.33 \mathrm{~g} \\
1026 \mathrm{~kg} / \mathrm{m}^{3} \\
1.2 \times 10^{-3} \mathrm{~Pa} \cdot \mathrm{s} \\
17.35 \mathrm{~mm}\end{array}$} \\
\hline & Linear density & \\
\hline & Rotational stiffness & \\
\hline & Rotational damping & \\
\hline \multirow{2}{*}{ Towed body } & Diameter & \\
\hline & Mass & \\
\hline \multirow{2}{*}{ Water } & Density & \\
\hline & Viscosity & \\
\hline Winch & Radius & \\
\hline
\end{tabular}

Table 3.1: Towed body simulator system parameters [1].

The previous work [1] measured the flow variation with depth that was present in the flume scale experiment and determined an empirical linear relationship between the mean flow velocity $\overline{U_{x}}(z)$ and depth $z$ to be

$$
\overline{U_{x}}(z)=-0.5873 z-0.2304
$$

The flow vector $\overline{U_{x}}$ is decomposed into the cable linkages's body-fixed coordinate frame. $\overline{U_{y}(z)}$ and $\overline{U_{z}(z)}$, the mean flow velocities in the world frame $y$ and $z$ directions, are set to zero. Flow variance was also measured and modeled in the simulator as 


\subsection{WAVE MODELING AND SHIP MOTION}

Gaussian white noise with standard deviations in the $\mathrm{x}, \mathrm{y}$ and $\mathrm{z}$ directions to be $0.0300 \mathrm{~m} / \mathrm{s}, 0.0262 \mathrm{~m} / \mathrm{s}$ and $0.0152 \mathrm{~m} / \mathrm{s}$ respectively [1]

The cable is reeled in and out via a prismatic joint that joins the first cable segment to the sheave. In the previously developed computer simulator [29] a PD controller provides position control of the winch system that outputs the amount of cable to reel in or out based on the selected set point algorithm. A state space system of a DC motor was used to model the winch motor to output a rotational acceleration. The controller gains were tuned using the Simulink PID autotune feature based to obtain a $90 \%$ rise time in 0.12 seconds without overshoot. For the current study, a simplified winch model is used in which a PID controller outputs the amount of cable to reel in and out directly to the prismatic joint connected to the first cable segment. The following sections will outline the changes and additions made to Calnan et al.'s simulator in order for it to replicate a towed body in a lifting operation.

\subsection{Wave modeling and ship motion}

Previously digitized ship motion data from the Australian Defense Science and Technology Organization (DSTO) report was used. The six degrees of freedom motion profiles were resolved into three translational degrees of freedom and scaled to \pm 4 $\mathrm{cm}$ and presented in Figure 3.3 for the displacement time histories of the $x, y$ and $z$ directions. The same ship motion profiles are used in the flume scale simulations in the current thesis such that the results of Calnan's heave compensation efforts can be compared to when waves are added to the system.

Wave motion was added to the simulator in order to investigate wave synchronization during breach. Both regular and irregular waves were modelled for sea states 3 through 6 which are based on the scaled significant wave heights $H_{s}$ and

modal periods $T_{p}$. The values of $H_{s}$ and $T_{p}$ for the sea states can be found in Table 2.2. Regular waves were modelled using linear wave theory, with the displacement of 

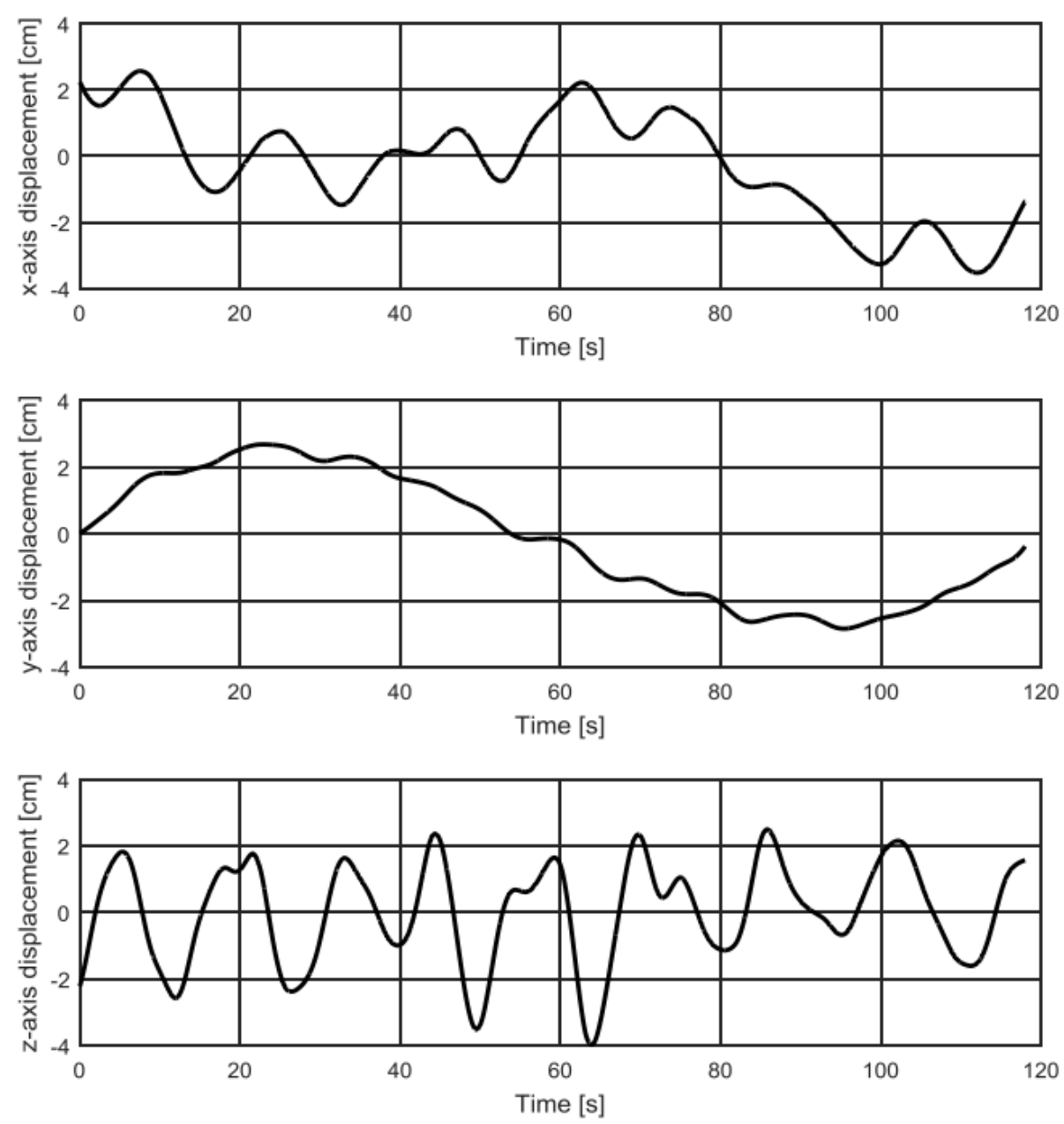

Figure 3.3: Resolved 3 degrees of freedom ship motion data scaled to $\pm 4 \mathrm{~cm}$ from the Australian DSTO report. Reproduced from [1].

the free water surface $\eta(x, t)$ given by

$$
\eta\left(x_{T B}, t\right)=\frac{1}{2} H_{s} \cos \left(k_{p} x_{T B}-\frac{2 \pi}{T_{p}} t\right)
$$

where $x_{T B}$ is the position of the towed body in the world frame $x$ direction and $k_{p}$ is the progressive wave number. Irregular waves were modelled from the Bretschneider wave spectrum, with the wave profile given in Equation 2.17.

One must not only account for the surface waves but also the fluid motion below the waterline as the fluid/wave action decays exponentially with depth. The 


\subsection{EXTERNAL FORCES}

factor $\kappa(z)$, given by Sagutun et al [2] in Equation 2.28, is applied over the entire flume tank depth in the simulator and changed to the simulator coordinate system, such that

$$
\kappa\left(z_{T B}\right)= \begin{cases}e^{k_{p}\left(z_{T B}\right)} & z_{T B}<0 \\ 1 & z_{T B} \geq 0\end{cases}
$$

The vertical velocity of the wave motion, $\kappa\left(z_{T B}\right) \dot{\eta}\left(x_{T B}, t\right)$, is added to the vertical flow velocity in the flume tank $U_{o, z}$ that is used in the derivation of external forces discussed in the next section.

\subsection{External forces}

The hydrodynamic forces acting on the cable and towed body inline with the flow in Calnan et al.'s simulator can be summarized by the Morison equation which models the forces acting on a body in normal, non-uniform flow [34]. For flow that is decomposed into the body-fixed coordinate frame of the cable segments and towed body, the Morison equation is expressed for each axis as

$$
f_{e x t, x, y, z}=\frac{1}{2} \rho C_{D, x, y, z} A_{p, x, y, z}\left|V_{x, y, z}\right| V_{x, y, z}+\rho C_{A} V \dot{V}_{x, y, z}+\rho V \dot{U}_{o, x, y, z}
$$

where $\rho$ is the density of the fluid, $C_{D, x, y, z}$ is the normal drag coefficient for the three primary axes, $A_{p, x, y, z}$ is the projected frontal area of the body in each direction of the body-fixed coordinate frame, $V_{x, y, z}$ is the relative velocity between the body and fluid flow, $U_{o, x, y, z}$ is the flow velocity in each direction of the body-fixed coordinate frame, $C_{A}$ is the added mass coefficient and $V$ is the volume of the body. The first term of Equation 3.4 represents the viscous drag force on the body. The drag coefficients $C_{D, x, y, z}$ for the cable and towed body are assumed to be constant as a function of space and time. The second term of Equation 3.4 is the hydrodynamic mass force representing the inertia added to the system by the surrounding fluid as it moves with 
the body [25]. The third term is the Froude-Krylov pressure force, as per Equation 2.25. The Froude-Krylov force represents the force created by an unsteady pressure field generated by undisturbed waves [11]. In Calnan's simulator, the assumption of steady flow is applied such that the Froude-Krylov force becomes zero. As the towed body sphere is very small in this study, drag forces will dominate the inertia forces and thus the Froude-Krylov force can also be neglected.

In Calnan et al.'s numerical model the added mass of the cylindrical cable segments $m_{C A}$ and the towed sphere $m_{S A}$ are obtained from the following two expressions respectively

$$
\begin{aligned}
m_{C A} & =\rho \pi l_{i}{\frac{d_{c}{ }^{2}}{2}}_{m_{S A}}=\frac{2}{3} \rho \pi{\frac{d_{T B}}{2}}^{3}
\end{aligned}
$$

where $l_{i}$ is the length of each cable segment and $d_{c}$ and $d_{T B}$ are the diameters of the cable and sphere respectively. The values of $l_{i}, d_{c}$ and $d_{T B}$ are found in Table 3.1. The added mass values, $m_{C A}$ and $m_{S A}$, correspond to the mass of the fluid displaced by the body and are added to the inertial mass of the cable segment and towed body in the simulator [1]. From Equations 3.5 and 3.6, values of $0.17 \mathrm{~g} / \mathrm{m}$ and $0.26 \mathrm{~g}$ for the added mass of the cable and towed body respectively were obtained [26, 29].

Buoyancy and gravitational forces also act on the cable and towed body in the $z$ direction of the world frame. The gravitational force $F_{W}$ acts in the negative $z$ direction in the world frame and is a function of mass and gravitational acceleration $g$. The buoyancy force $F_{b}$ acts in the positive $z$ direction and is equated to the weight of the displaced fluid. The buoyancy is a function of the displaced volume $V_{\text {sub }}$ and density of the fluid $\rho$ and is given by

$$
F_{b}=\rho g \forall_{s u b}
$$

In the simulator, the external forces acting on the cable and towed body, 


\subsection{EXTERNAL FORCES}

as described above, are applied at the centre of gravity of each rigid body of the Simulink model [1]. Buoyancy and drag are only applied to components that are submerged. During breach, the towed body and cable elements are exiting the water and possibly re-entering the water where elements are not fully breached. In Calnan et al.'s model, the water exit is accounted for by setting the viscous drag and buoyancy forces on a body to zero when the centre of gravity of any rigid body is detected above the waterline. This step function definition of the drag and buoyancy forces was judged to be sufficient for the heave compensation application where the majority of the model elements remain fully submerged during operation. However, during a recovery operation, all elements will eventually exit the water. In the current thesis work, a simple "on/off" approach to modelling the external loads on a body does not adequately model the dynamic breach phase, where the bodies are partially submerged and still experience the effects of buoyancy and drag. Thus, improvements were made in the simulator to model the external loads on the towed body during breach.

\subsubsection{Breach}

Figure 3.4 illustrates the change in submerged volume and wetted area of the towed body as it exits the water. External forces acting on the towed body in the $x-z$ plane are shown where only the viscous drag in the $x$ direction is depicted. The solid area refers to the portion of the towed body that is submerged and the hatched area refers to the portion of the towed body that is not submerged. The geometry in Figure 3.4 is the same for the forces acting in the $y-z$ plane, which are not shown. The position of the towed body centre of gravity in the world frame $z$ direction is $z_{T B}$, and $r_{T B}$ is the radius of the towed body. The parameter $h$ describes the height of the sphere that is submerged, with $h$ described by the interval $h \in\left[0,2 r_{T B}\right]$.

The start of the breach phase is defined as the point in time when the towed body just begins to exit the water, that is when $z_{T B}=-r_{T B}$. Similarly, 


\section{CHAPTER 3. WAVE SYNCHRONIZATION MODEL AND SIMULATION}

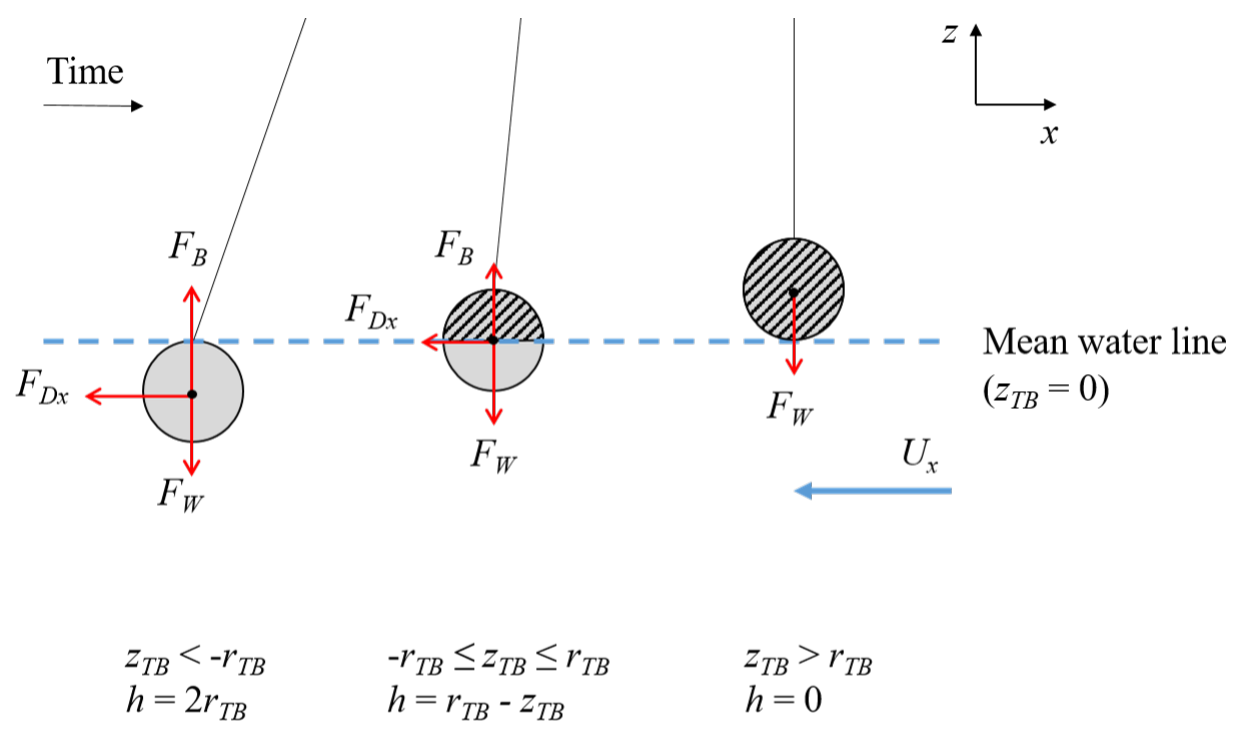

Figure 3.4: Change in submerged volume and wetted area of the towed body as the body is removed from the water during breach. Geometry is shown for forces acting on the towed body in the $x-z$ plane and is the same for forces acting in the $y-z$ plane.

the towed body is defined to have fully breached when $z_{T B}=r_{T B}$. The buoyancy force during breach is therefore a function of the change in submerged volume over the interval $z_{T B} \in\left[-r_{T B}, r_{T B}\right]$. Figure 3.5 illustrates the parameters used in the derivation of the volume of a partially submerged sphere $V_{\text {breach }}$ which is obtained by summing the volumes of cylinders with infinitesimal thickness $d h$ and radius $x=$ $\sqrt{r_{T B}^{2}-\left(r_{T B}-h\right)^{2}}$ over the interval $h \epsilon[0,2 r]$.

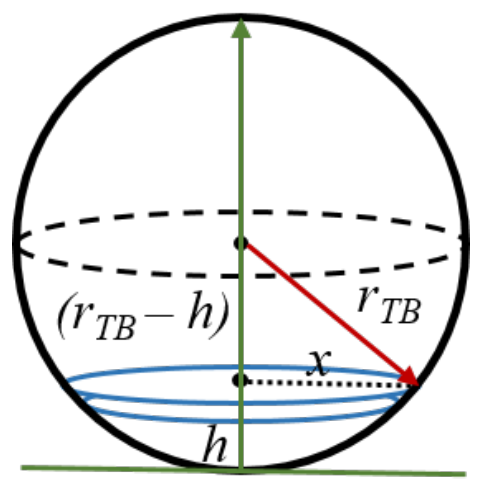

Figure 3.5: Parameters used in the derivation of volume of a partially submerged sphere where $h \in\left[0,2 r_{T B}\right]$ and $x=\sqrt{r_{T B}^{2}-\left(r_{T B}-h\right)^{2}}$. 


\subsection{EXTERNAL FORCES}

The equation to obtain the submerged volume of the towed body during breach is therefore

$$
V_{\text {breach }}=\int_{0}^{h} \pi\left(r_{T B}^{2}-\left(r_{T B}-h\right)^{2}\right) d h .
$$

The buoyancy force can now be fully defined by the following piecewise function:

$$
F_{b}=\left\{\begin{array}{ll}
\rho g V_{\text {sub }} & z_{T B}<-r_{T B} \\
\rho g V_{\text {breach }} & -r_{T B} \leq z_{T B} \leq r_{T B} \\
0 & z_{T B}>r_{T B}
\end{array} .\right.
$$

The viscous drag force on the submerged body is also a function of the payload position during breach, due to the change in the wetted frontal area $A_{p, x, y, z}$. Referring to the middle image of Figure 3.4, the frontal area of the partially submerged towed body in the $x$ and $y$ directions can be described as the area of a segment of a circle and, following the derivation in [35], is expressed as

$$
A_{p, x, y}=r_{T B}^{2} \cos ^{-1}\left(\frac{r_{T B}-h}{r_{T B}}\right)-\left(r_{T B}-h\right) \sqrt{2 r_{T B} h-h^{2}}
$$

over the interval $h \epsilon\left[0,2 r_{T B}\right]$ with $h$ defined in Figure 3.5. The wetted frontal area in the $z$ direction is defined differently. Figure 3.6 depicts three stages of the breach phase as the towed body exits the water. External forces acting on the towed body in the $x-z$ plane are shown in the front view, where only the viscous drag in the $z$ direction is depicted. The solid area refers to the portion of the towed body that is submerged and the hatched area refers to the portion of the towed body that is not submerged. The bottom view displays the change in the wetted area of the towed body projected onto the $x-y$ plane. Here, the wetted area is a circle with a radius $R$ equal to half the chord length of the submerged section that changes with the position of the towed body.

The cord length radius $R$, also derived in [35], and by extension the area of 
CHAPTER 3. WAVE SYNCHRONIZATION MODEL AND SIMULATION

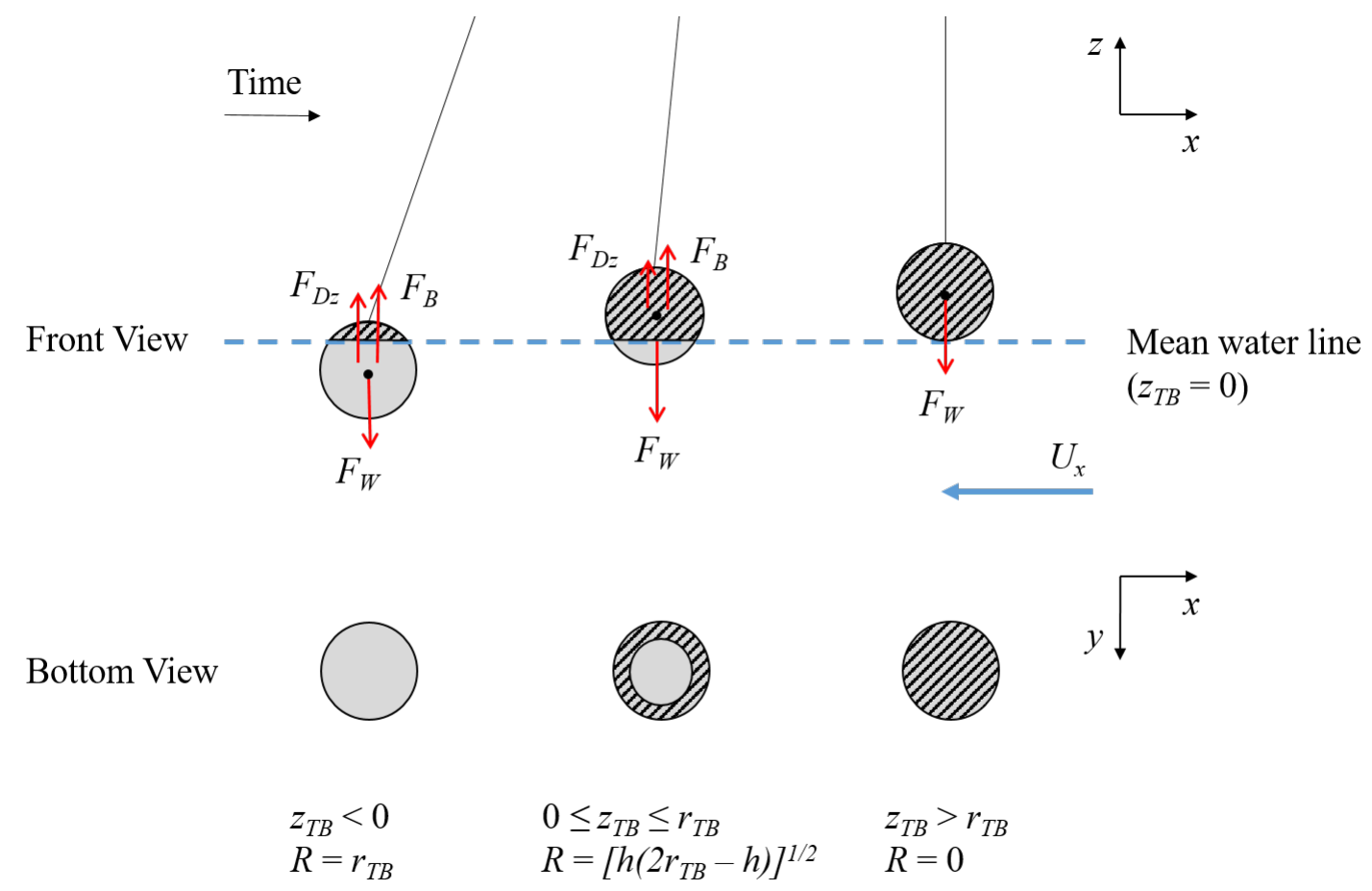

Figure 3.6: Front and bottom views displaying the change in wetted area of the towed body. The bottom view is projected onto the $x-y$ plane that is perpendicular to the flow in the $z$ direction.

the projected circle $A_{p, z}$ are expressed respectively as,

$$
\begin{gathered}
R=\sqrt{h\left(2 r_{T B}-h\right)}, \\
A_{p, z}=\pi h\left(2 r_{T B}-h\right) .
\end{gathered}
$$

Finally, Equations 3.10 and 3.12 are substituted into the viscous drag term in the Morison equation (Equation 3.4) to compute the external forces on the cable and towed body. The drag coefficients $C_{D, x, y, z}$ in the Morison equation are dependent on the orientation of the $x, y, z$ axes in the body-fixed coordinate frame of the rigid bodies. Drag coefficients for flow that is normal to the submerged bodies, i.e. in the $x$ and $y$ directions for the cable and in all directions for the towed sphere, were found in the literature to be 1 and 0.5 for the cable and towed body respectively [36], which are consistent with the values used by Calnan et al. The $z$ axis in the cable body fixed coordinate frames is tangential to the cable segment and drag acting along this 


\subsection{COMPENSATOR STRATEGIES}

axis is due to skin friction. This tangential drag coefficient is approximated by 0.01 [36].

Hydrodynamic exit and slamming loads are also applied in the world frame $z$ direction to the towed body centre of gravity as it exits and reenters the water throughout the breach phase. Hydrodynamic exit and slamming loads are defined as the rate of change of fluid kinetic energy and are related to the change in the added mass of the body as it exits and enters the water [26]. The water exit force $F_{e}$ on a fully submerged object lifted up beneath the free surface at a constant lifting velocity $v_{e}$ is given by

$$
F_{e}=-\frac{1}{2} \rho C_{e} A_{p, z} v_{e}^{2}
$$

where $C_{e}$ is the water exit coefficient for a sphere. Similarly, the slamming load $F_{s}$, directed in the positive world frame $z$ direction, is given by [26]

$$
F_{s}=\frac{1}{2} \rho C_{s} A_{p, z} v_{e}^{2}
$$

where $C_{s}$ is the slamming coefficient. Both the water exit coefficient $C_{e}$ and slamming coefficient $C_{s}$ are related to the change of added mass with submergence. As the added mass is assumed to be constant in the scope of this thesis, the coefficients $C_{e}$ and $C_{s}$ for a sphere are also constant and are set to 0.5 [26].

With the external forces on the payload during breach defined, Calnan's simulator was further advanced by updating the active heave compensator set-point algorithms to include wave synchronization.

\subsection{Compensator strategies}

Calnan et al.'s two principle AHC set-point algorithms were amended to account for the motion imparted to the cable and towed body by the waves. The waterline method is based on reeling in and out the cable to ensure that the same point along 


\section{CHAPTER 3. WAVE SYNCHRONIZATION MODEL AND SIMULATION}

the cable crosses the static waterline. With surface waves, the cable and towed body enter and exit the water at the position $\eta$ above or below the mean waterline. To maintain the same water entry point along the cable, the water surface elevation must be subtracted from the sheave height to the mean waterline which effectively synchronizes the towed body motion with that of the surface waves. Figure 3.7 depicts the waterline set-point algorithm with wave synchronization, where $\mathrm{H}$ is the height of the sheave above the water surface. The amended set-point for the length of cable to be reeled in $L_{S P}$ is expressed as

$$
L_{S P}=\frac{H-\eta}{\cos \theta_{\text {nom }}}-\frac{H_{\text {nom }}-\eta}{\cos \theta_{\text {nom }}} .
$$

The nominal sheave angle $\theta_{\text {nom }}$ describes the nominal angle that the cable enters the water during the towed operation. When the sheave angle $\theta$ is measured directly, then the algorithm is referred to as rigorous. If the sheave angle is not measured, then it is referred to as simplified and nominal sheave angle $\theta_{\text {nom }}$ is used. Only the simplified waterline method will be applied in the simulations in this work as the rigorous waterline method was found to be unreliable and showed a high degree of sensitivity to the actual sheave angle $\theta$ and sheave height above the static waterline $[29]$.

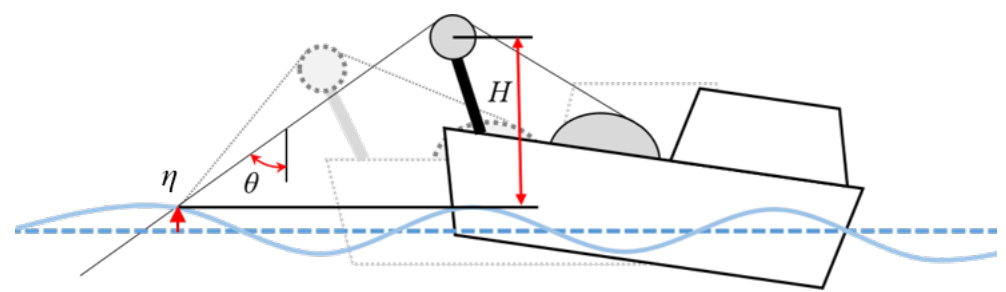

Figure 3.7: Waterline set-point algorithm with wave synchronization.

The sheave set-point algorithm determines the desired cable adjustment based on sheave displacement. To advance the sheave set-point algorithm, the water surface elevation is subtracted from the vertical sheave motion. Similar to the waterline set-point algorithm adjustment, subtracting the surface elevation is expected to have effect of synchronizing the vertical towed body motion with the wave motion. 


\subsection{SIMULATION RESULTS}

Figure 3.8 depicts the sheave set-point algorithm with wave synchronization, where $\Delta x$ and $\Delta z$ are the $x$ and $z$ sheave displacements respectively. The adjusted set-point algorithm for the desired cable length adjustment $L_{S P}$ is given by

$$
L_{S P}=(\Delta x) \sin \theta+(\Delta z-\eta) \cos \theta
$$

Only the rigorous sheave method will be applied in the simulations in this work. The simplified sheave method will be excluded from this study as it was found to behave similarly to the simplified waterline method [29]. Thus only the simple waterline and the rigorous sheave will be advanced and investigated in the following simulations.

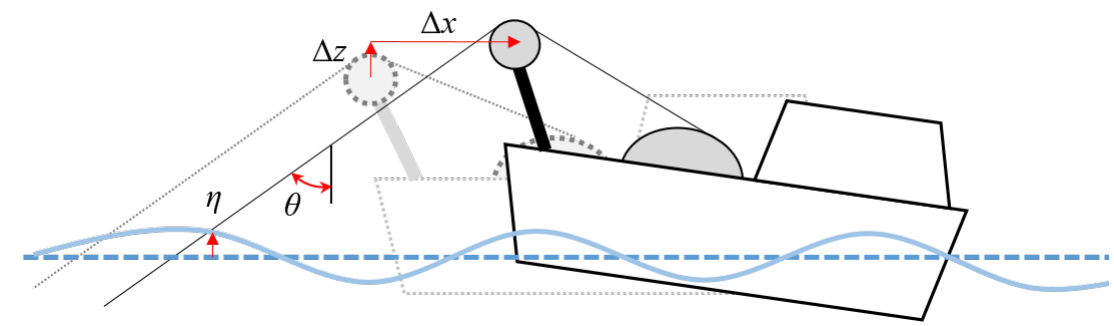

Figure 3.8: Sheave set-point algorithm with wave synchronization.

\subsection{Simulation results}

In the effort to investigate the usefulness of combining active heave compensation with wave synchronization in recovery operations, simulations were run for three compensation strategies: combined heave compensation with wave synchronization, only heave compensation, and no control. Both the simplified waterline method and rigorous sheave set-point algorithms were used in the strategies containing heave compensation. All three compensator strategies were run with regular and irregular waves in sea states 3 through 6 . In all simulations, the towed body started at a depth of $20 \mathrm{~cm}$ below the mean still water line and was reeled in at a constant speed of $0.021 \mathrm{~m} / \mathrm{s}$. Simulations were run for $15 \mathrm{~s}$ in the Simulink environment. 


\section{CHAPTER 3. WAVE SYNCHRONIZATION MODEL AND SIMULATION}

In accordance with Sagutun et al. [2], the performance of each compensator strategy is evaluated based on cable tension observed throughout each simulation. Table 3.2 summarizes the mean and standard deviation of cable tension for the combined heave compensation and wave synchronization strategies using the rigorous sheave (RS) and simplified waterline (W) set-point algorithms, with $\theta_{\text {nom }}$ set as $50^{\circ}$ as selected by Calnan et al. [29], and the cable tension observed in the case of no control (Null). Values are tabulated for each sea state with both regular and irregular waves. The mean and standard deviation of the cable tension were computed on the interval $t=[0.5,15] \mathrm{s}$ in order to reject the values observed while the model initialized. From Table 3.2, little difference is observed between the combined compensator methods using the rigorous sheave and waterline set-point algorithms. The simulations run with no control strategy appear to perform better than those run with the combined strategy as the standard deviation of tension is generally lower than the other cases.

\begin{tabular}{|c|l|l|l|l|l|l|l|l|l|}
\cline { 2 - 10 } \multicolumn{2}{c|}{} & \multicolumn{9}{c|}{ Sea State } \\
\cline { 2 - 11 } \multicolumn{2}{c|}{ SS3 } & \multicolumn{2}{c|}{ SS4 } & \multicolumn{2}{c|}{ SS5 } & \multicolumn{2}{c|}{ SS6 } \\
\hline \multirow{2}{*}{ Method } & Reg. & Irreg. & Reg. & Irreg. & Reg. & Irreg. & Reg. & Irreg. \\
\hline \multirow{2}{*}{ RS } & Mean & 1.05 & 1.05 & 1.05 & 1.05 & 1.06 & 1.06 & 1.06 & 1.05 \\
\cline { 2 - 11 } & Std Dev & 0.239 & 0.237 & 0.243 & 0.239 & 0.264 & 0.249 & 0.29 & 0.268 \\
\hline \multirow{2}{*}{ W } & Mean & 1.06 & 1.06 & 1.06 & 1.06 & 1.06 & 1.06 & 1.07 & 1.06 \\
\cline { 2 - 10 } & Std Dev & 0.240 & 0.24 & 0.249 & 0.242 & 0.255 & 0.251 & 0.299 & 0.266 \\
\hline \multirow{2}{*}{ Null } & Mean & 0.991 & 0.989 & 0.988 & 0.989 & 0.993 & 0.99 & 0.999 & 0.995 \\
\cline { 2 - 9 } & Std Dev & 0.216 & 0.21 & 0.215 & 0.212 & 0.227 & 0.217 & 0.231 & 0.225 \\
\hline
\end{tabular}

Table 3.2: Cable tension results for the combined wave synchronization and AHC strategy compared to simulations with no control method implemented. The mean and standard deviation of the cable tension throughout were computed on the interval $t=[0.5,15] \mathrm{s}$. All values are in $\times 10^{-2}$ Newtons.

Figure 3.9 graphs the information contained in Table 3.2 with mean cable tension plotted in subfigures $3.9 \mathrm{a}$ to $3.9 \mathrm{~d}$ and standard deviation of cable tension represented by error bars. The results from simulations run with regular waves are displayed as solid bars, and the results from simulations run with irregular waves are displayed as hatched bars. Visually, it is shown that using the combined heave compensation and wave synchronization strategy with either set-point algorithm does not perform better than having no control at all based on the metric of cable tension. 


\subsection{SIMULATION RESULTS}

Additionally, as there exists no significant difference between the results of the combined control strategy with either the rigorous sheave or simplified waterline set-point algorithms, with the rigorous sheave performing slightly better than the latter, only the simulations run using the rigorous sheave set-point algorithms will be considered in the remaining analyses.

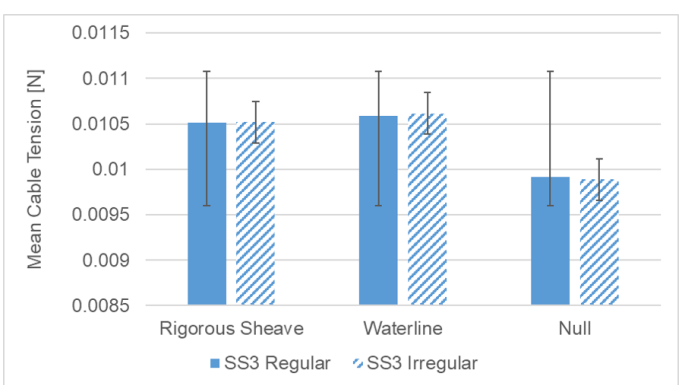

(a) SS3

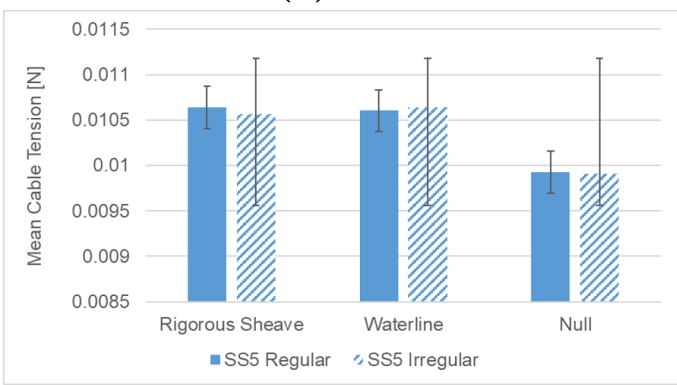

(c) SS5

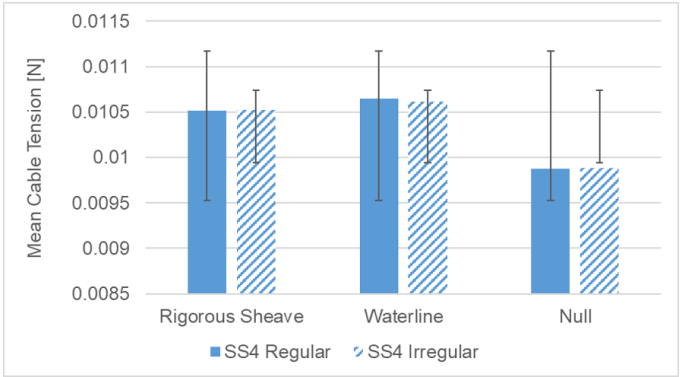

(b) SS4

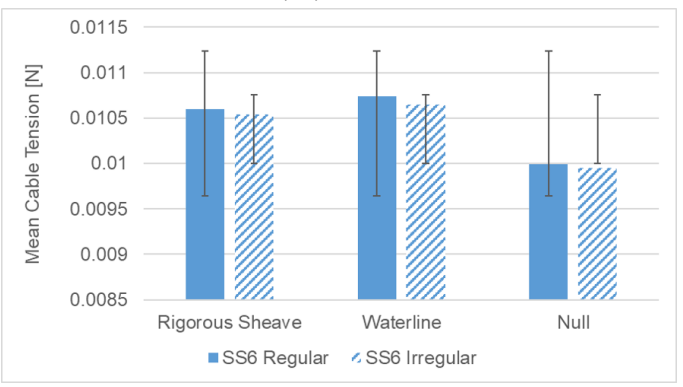

(d) SS6

Figure 3.9: Mean cable tension measured during simulations that were run with the combined wave synchronization and AHC strategies compared to the simulations run with no control for sea states a)3, b)4, c)5 and d)6. Error bars represent 2 standard deviations.

To quantify the effects of combining wave synchronization with heave compensation over using only heave compensation, both methods were compared using the rigorous sheave set-point algorithm. Table 3.3 summarizes the mean and standard deviation of cable tension for the combined heave compensation and wave synchronization strategy (AHC + WS), the heave compensation strategy (AHC) and the cable tension observed in the case of no control (Null). Values are tabulated for each sea state with both regular and irregular waves. As before, the mean and standard 
deviation of the cable tension were computed on the interval $t=[0.5,15] \mathrm{s}$ in order to reject the values observed while the model initialized. From Table 3.3, no significant difference is observed between the combined compensator method and the heave compensation method. Similar to the results from Table 3.2, the simulations run with no control strategy appear to perform better than either motion compensation control strategies.

\begin{tabular}{|c|c|c|c|c|c|c|c|c|c|}
\hline & \multicolumn{8}{|c|}{ Sea State } \\
\hline & & \multicolumn{2}{|c|}{ SS3 } & \multicolumn{2}{|c|}{ SS4 } & \multicolumn{2}{|c|}{ SS5 } & \multicolumn{2}{|c|}{ SS6 } \\
\hline \multicolumn{2}{|c|}{ RS Method } & Reg. & Irreg. & Reg. & Irreg. & Reg. & Irreg. & Reg. & Irreg. \\
\hline \multirow{2}{*}{$\begin{array}{r}\mathrm{AHC}+ \\
\mathrm{WS}\end{array}$} & Mean & 1.05 & 1.05 & 1.05 & 1.05 & 1.06 & 1.06 & 1.06 & 1.05 \\
\hline & Std Dev & 0.239 & 0.237 & 0.243 & 0.239 & 0.264 & 0.249 & 0.29 & 0.268 \\
\hline \multirow{2}{*}{$\mathrm{AHC}$} & Mean & 1.05 & 1.05 & 1.05 & 1.05 & 1.05 & 1.05 & 1.06 & 0.989 \\
\hline & Std Dev & 0.239 & 0.236 & 0.241 & 0.239 & 0.250 & 0.243 & 0.253 & 0.21 \\
\hline \multirow{2}{*}{ Null } & Mean & 0.991 & 0.989 & 0.988 & 0.989 & 0.993 & 0.99 & 0.999 & 0.995 \\
\hline & Std Dev & 0.216 & 0.21 & 0.215 & 0.212 & 0.227 & 0.217 & 0.231 & 0.225 \\
\hline
\end{tabular}

Table 3.3: Cable tension results for the combined wave synchronization and AHC strategy compared to simulations with only heave compensation applied. The mean and standard deviation of the cable tension throughout were computed on the interval $t=[0.5,15] \mathrm{s}$. All values are in $\times 10^{-2}$ Newtons.

Figures 3.10 graph the information contained in Table 3.3 with mean cable tension plotted in subfigures $3.10 \mathrm{a}$ to $3.10 \mathrm{~d}$ and standard deviation of cable tension represented by error bars. The results from simulations run with regular waves are displayed as solid bars, and the results from simulations run with irregular waves are displayed as hatched bars. Visually, it appears that there exists no significant difference between the results of the combined control strategy and those of the heave compensation strategy.

Unlike the conclusions of Sagutun et al. [2] and Johansen et al. [5], where wave synchronization combined with a feedforward heave compensation strategy demonstrated a reduction in the standard deviation of cable tension in small-scale experimental moonpool operations, the addition of wave synchronization to Calnan et al.'s set-point algorithms was not found to provide any added benefit to the simulated cable tension during towed recovery operations. The recovery operation simulated in 


\subsection{SIMULATION RESULTS}

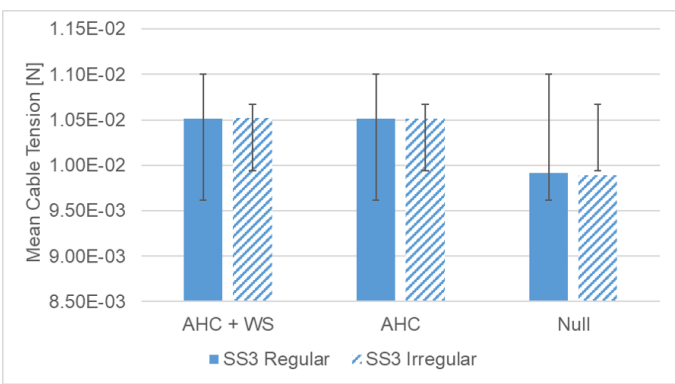

(a) SS3

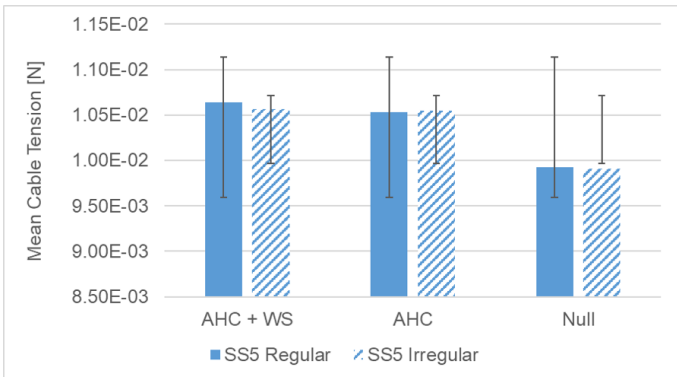

(c) SS5

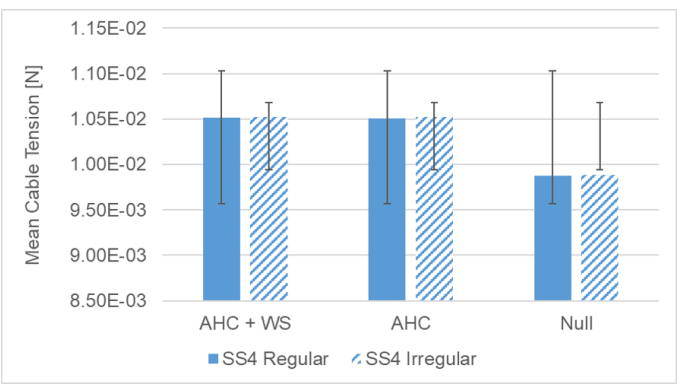

(b) SS4

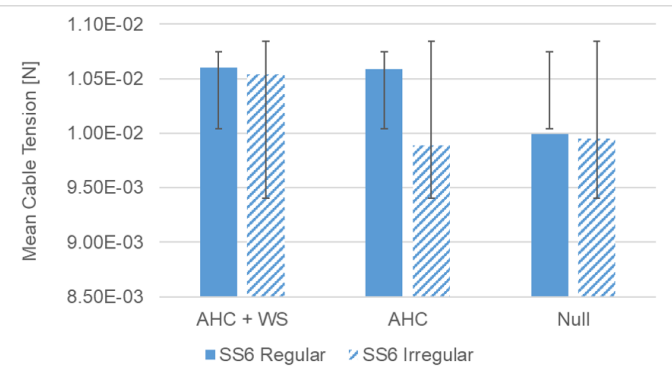

(d) SS6

Figure 3.10: Mean cable tension measured during simulations that were run with the combined wave synchronization and AHC strategy compared to the simulations run with only heave compensation for sea states a) 3 , b) 4 , c) 5 and d) 6 . Error bars represent 2 standard deviations.

this work differed from the moonpool operations investigated in the works of Sagutun et al. and Johansen et al. In the moonpool operations reproduced experimentally, the payload was lowered into the water, where the payload did not experience the effects of wave motion or any hydrodynamic loads when wave synchronization was applied. The recovery operations that were simulated in the current thesis, the payload, or towed body, was lifted out of the water, where the payload was experiencing hydrodynamic loads and motion induced by wave motion below the water surface when wave synchronization was applied. Furthermore, in moonpool operations, the moonpool in the hull of the host vessel acts like a piston in a cylinder, where the water vertical velocity is assumed to be approximately constant from the water surface to the bottom of the moonpool [5].

Further comparisons between the experimental results of Sagutun et al. and Johansen et al. and the simulation results obtained in this work can be made by 
qualitatively comparing the behaviour of both systems. Adapted from Sagutun et al. [2], Figure 3.11 shows the payload position and cable tension for the experimental test performed with regular waves at $T_{p}=1.25 \mathrm{~s}$ and $H_{s}=1.8 \mathrm{~cm}$. Subfigures 3.11a,c,e (right) plot the raw tension data, thin lines, and low-pass filtered tension data, thick lines, for experiments run using a combined heave compensation with wave synchronization strategy, heave compensation strategy and no control, respectively. With each strategy, cable tension is greatest when the body is above the water. In subfigures $3.11 b, d, f$ (left), the towed body position is plotted vs time for each control strategy. The towed body follows a vertical trajectory that approaches the mean water level located at $0 \mathrm{~m}$, where negative values mean the body is submerged.

Similar to Figure 3.11, Figures 3.12 and 3.13 plot the simulated cable tension and towed body vertical position vs time for regular waves at sea state $6, T_{p}$ $=1.03 \mathrm{~s}$ and $H_{s}=3.5 \mathrm{~cm}$, and at sea state $3, T_{p}=0.63 \mathrm{~s}$ and $H_{s}=0.7 \mathrm{~cm}$, respectively. Subfigures 3.12a,c,e and 3.13a,c,e (right) plot the cable tension for each control strategy. Like with the experimental results obtained by Sagutun et al., in every case cable tension is greatest when the towed body is above the water surface, where a sudden change in tension occurs when the towed body transitions from being submerged, to being suspended. In Figure 3.12a, particularly large fluctuations in the cable tension are observed before the towed body is fully breached. The large fluctuations in tension are a concern as cable detachment occurs when the tension becomes suddenly small and the cable loses contact with the sheave surface.

In subfigures $3.12 \mathrm{~b}, \mathrm{~d}, \mathrm{f}$ and $3.13 \mathrm{~b}, \mathrm{~d}, \mathrm{f}$ (left), the towed body position in the world frame $z$ direction is plotted vs time for each control strategy, where the towed body follows a trajectory that approaches the mean water level located at $0 \mathrm{~m}$ and where negative values indicate the towed body is submerged. The breach point is indicated in the position plots by a hexagram and is defined to occur when the towed body is completely above the water and does not re-submerge. Results obtained for simulation run with irregular waves at sea states 3 and 6 are included in Appendix A, where similar behaviour is observed. 


\subsection{SIMULATION RESULTS}

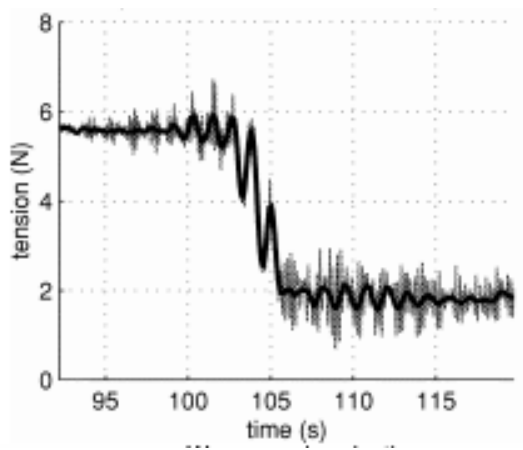

(a) Combined heave compensation and wave synchronization.

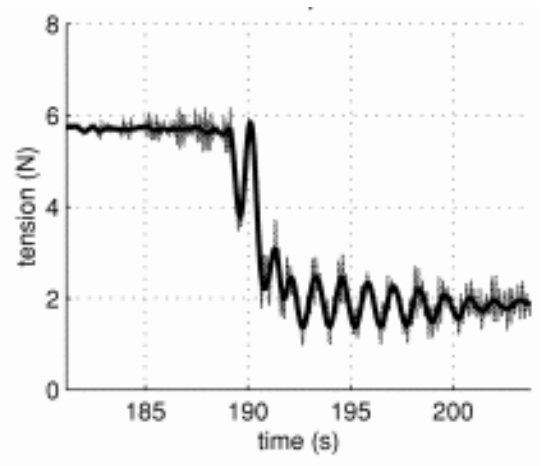

(c) Heave compensation.

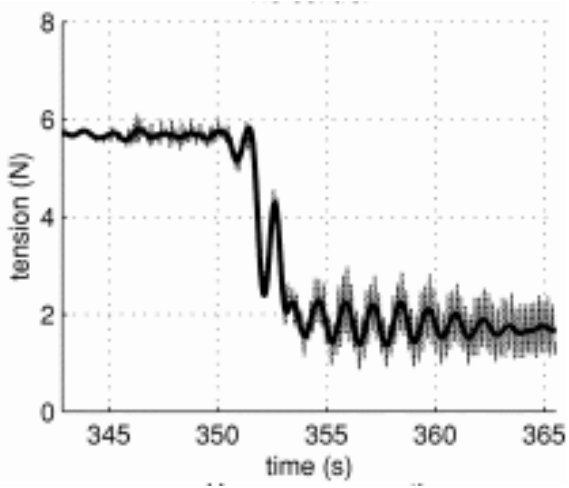

(e) Null control.

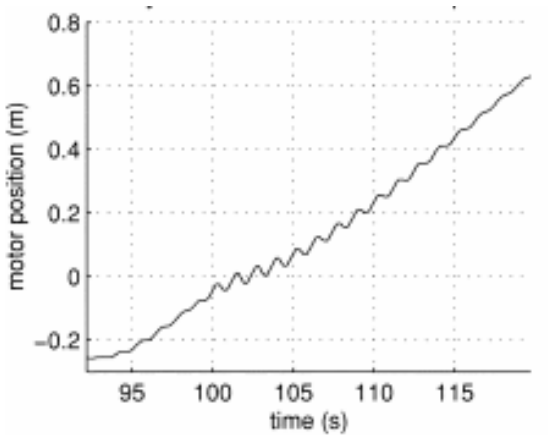

(b) Combined heave compensation and wave synchronization.

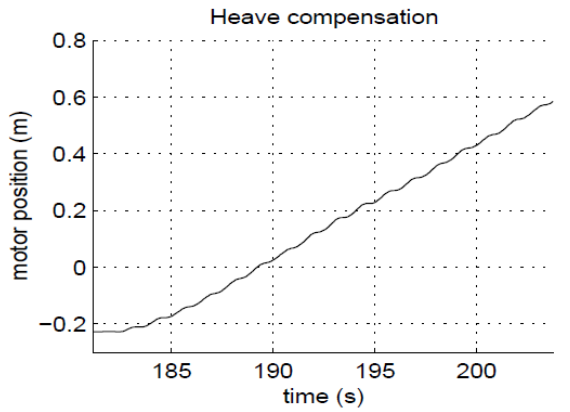

(d) Heave compensation

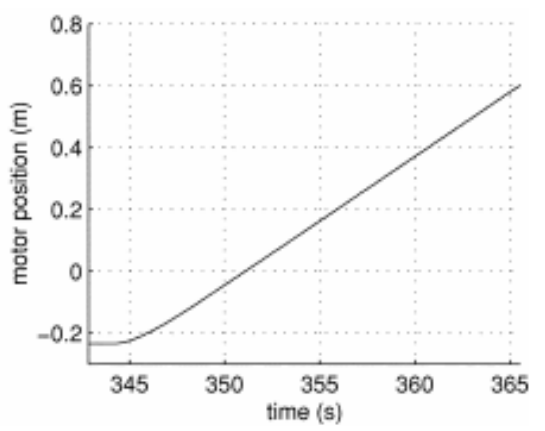

(f) Null Control

Figure 3.11: Adapted from Sagutun et al. [2] : Experimental results with regular waves at $T_{p}=1.25 \mathrm{~s}$ and $H_{s}=1.8 \mathrm{~cm}$, spherical payload. For the wire tension, we show both raw data (thin lines) and low-pass filtered data (thick lines). The payload position (measured using the motor shaft encoder) is in a vessel-fixed coordinate frame with zero at mean sea level.

In moonpool operations, the payload displacement is assumed to occur only in the vertical direction. In towing application, the towed body trajectory is more complex where the body can move in any direction. Figures 3.14 and 3.15 plot the 


\section{CHAPTER 3. WAVE SYNCHRONIZATION MODEL AND SIMULATION}

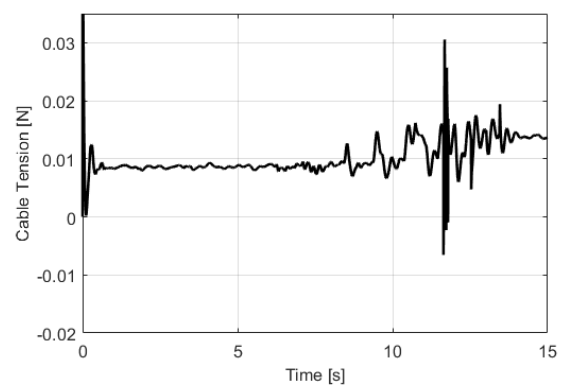

(a) Combined heave compensation and wave synchronization.

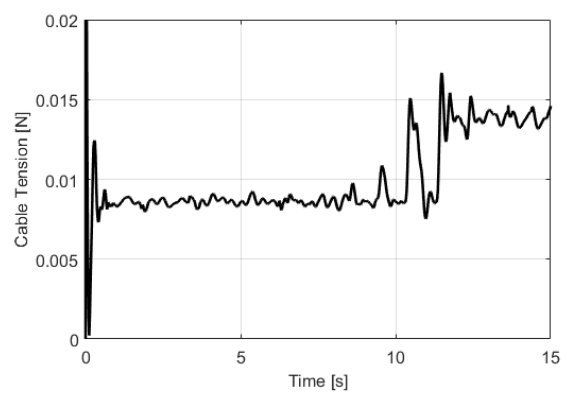

(c) Heave compensation.

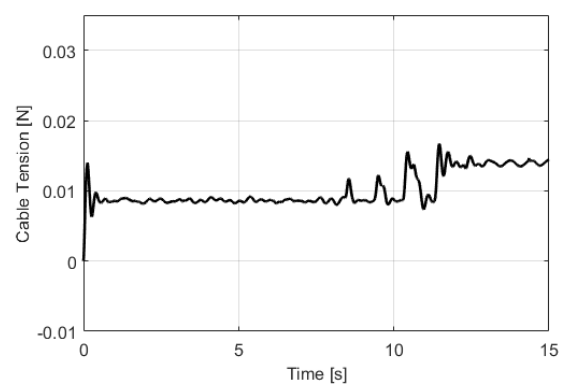

(e) Null control.

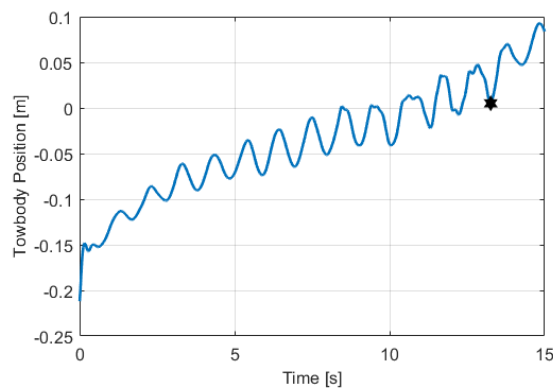

(b) Combined heave compensation and wave synchronization.

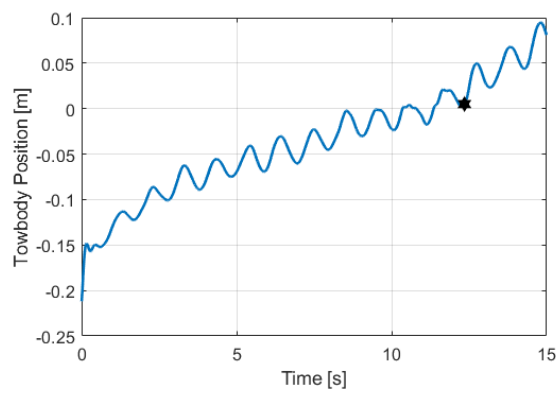

(d) Heave compensation

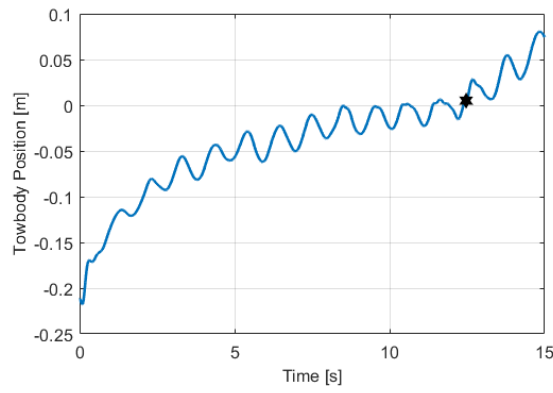

(f) Null Control

Figure 3.12: Simulated results with regular waves at SS6, $T_{p}=1.01 \mathrm{~s}$ and $H_{s}=$ $0.035 \mathrm{~m}$. Wire tension is shown on the left for a) combined heave compensation and wave synchronization, c) heave compensation only and e) no control. The towed body position in the world frame $z$ direction is shown on the right for b) combined heave compensation and wave synchronization, d) heave compensation only and f) no control. The towed body breach point is indicated by a hexagram in the towed body position figures.

towed body position in the $x, y$ and $z$ directions for simulations run using the combined wave synchronization and heave compensation control method for sea states 6 and 3 respectively. The wave motion is superimposed over the $z$ direction (vertical) trajectory where the breach point is marked by a hexagram. Towed body displace- 


\subsection{SIMULATION RESULTS}

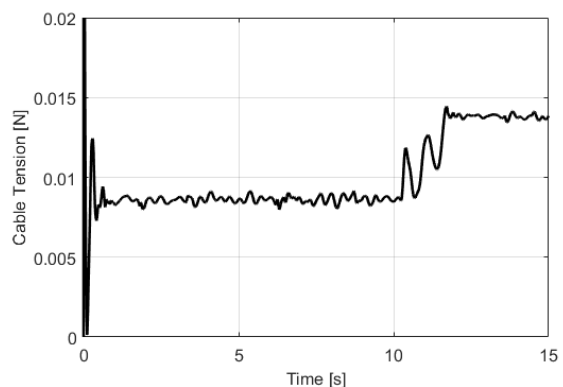

(a) Combined heave compensation and wave synchronization.

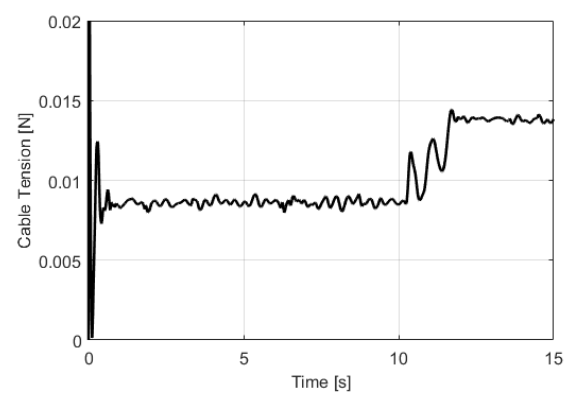

(c) Heave compensation.

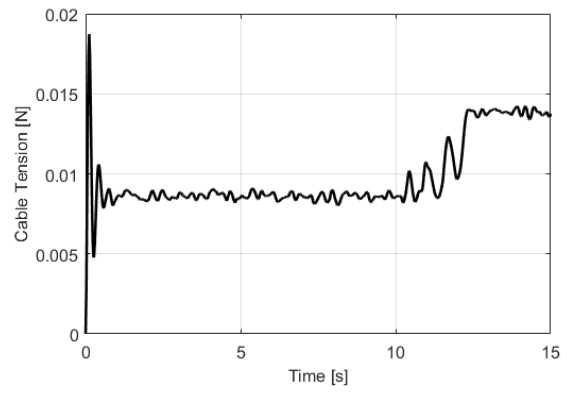

(e) Null control.

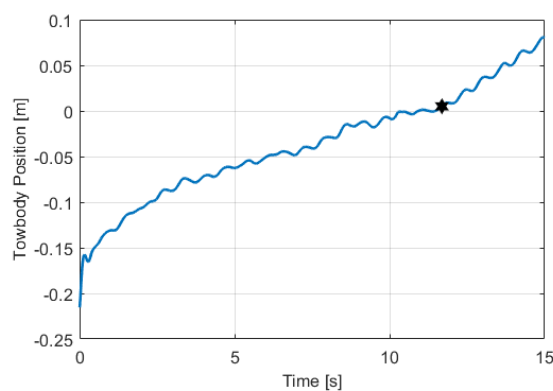

(b) Combined heave compensation and wave synchronization.

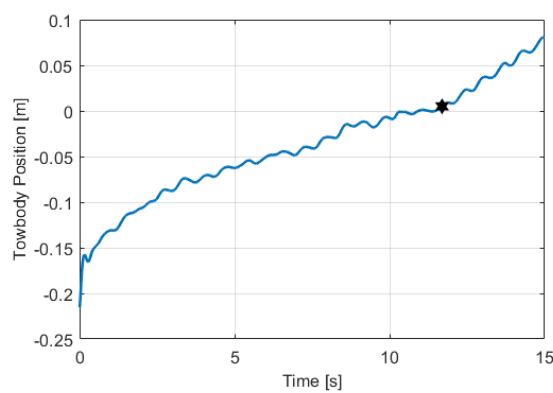

(d) Heave compensation

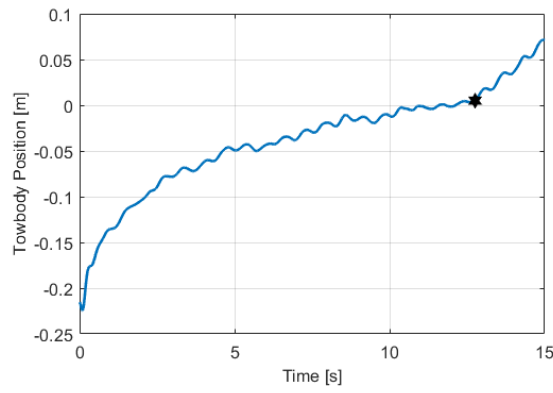

(f) Null Control

Figure 3.13: Simulated results with regular waves at $\mathrm{SS} 3, T_{p}=0.63 \mathrm{~s}$ and $H_{s}=$ $0.007 \mathrm{~m}$. Wire tension is shown on the left for a) combined heave compensation and wave synchronization, c) heave compensation only and e) no control. The towed body position in the world frame $z$ direction is shown on the right for b) combined heave compensation and wave synchronization, d) heave compensation only and f) no control. The towed body breach point is indicated by a hexagram in the towed body position figures.

ment in the $x$ direction, the direction of vessel motion, is significant when the towed body is above the water due to the pendulum motion induced by the removal of the hydrodynamic loads during breach. The pendulum motion that occurs when the towed body is suspended will be a focus of future work where motion compensation 


\section{CHAPTER 3. WAVE SYNCHRONIZATION MODEL AND SIMULATION}

strategies that minimize the pendulum motion will be investigated. The towed body position results obtained for simulations run with only heave compensation and no control in sea states 3 and 6 are included in Appendix A.
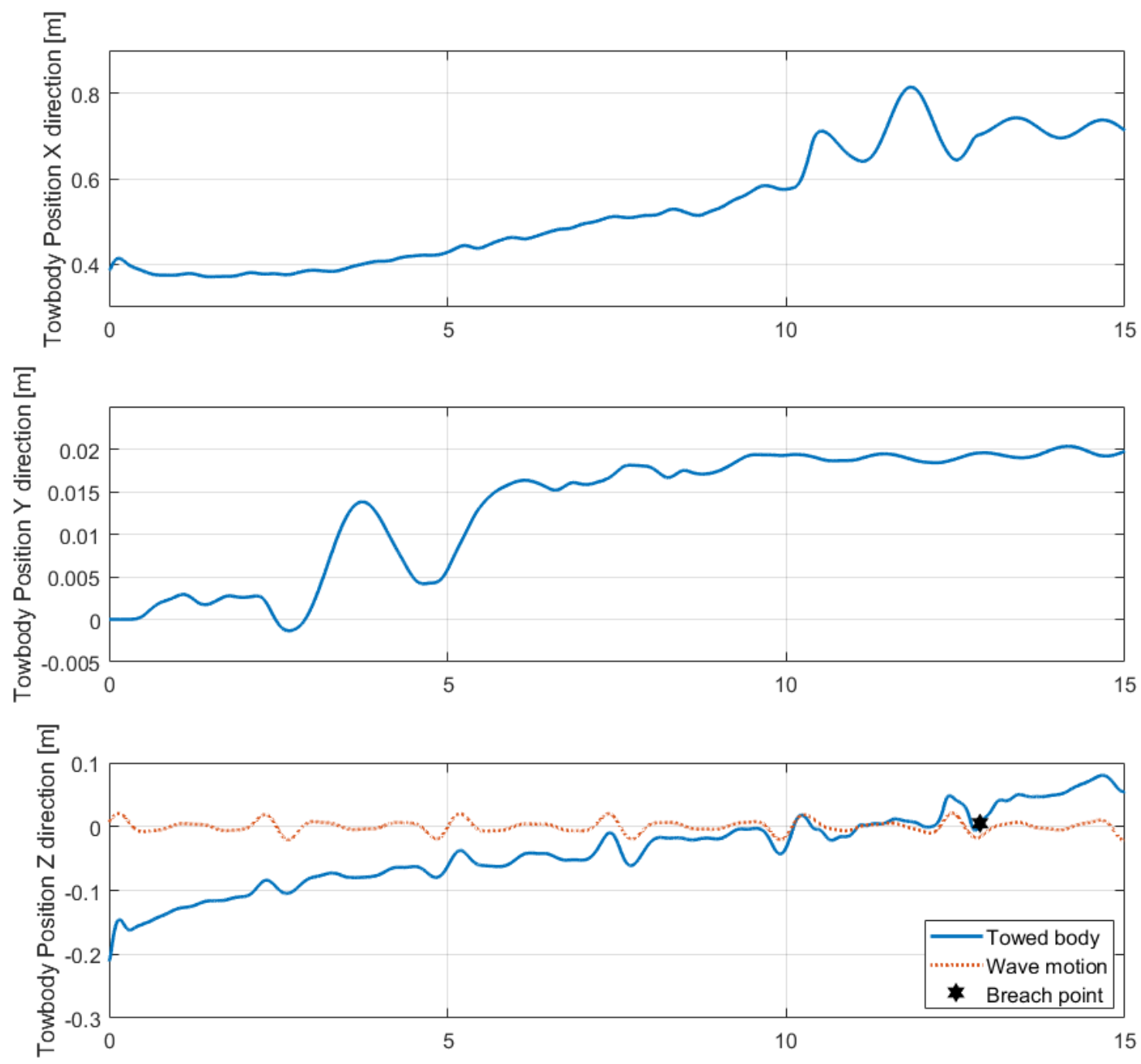

Figure 3.14: Simulated results for the towed body position with combined heave compensation and wave synchronization during a recovery operation with irregular waves at SS6. The towed body breach point is indicated by a hexagram. 


\subsection{SUMMARY}
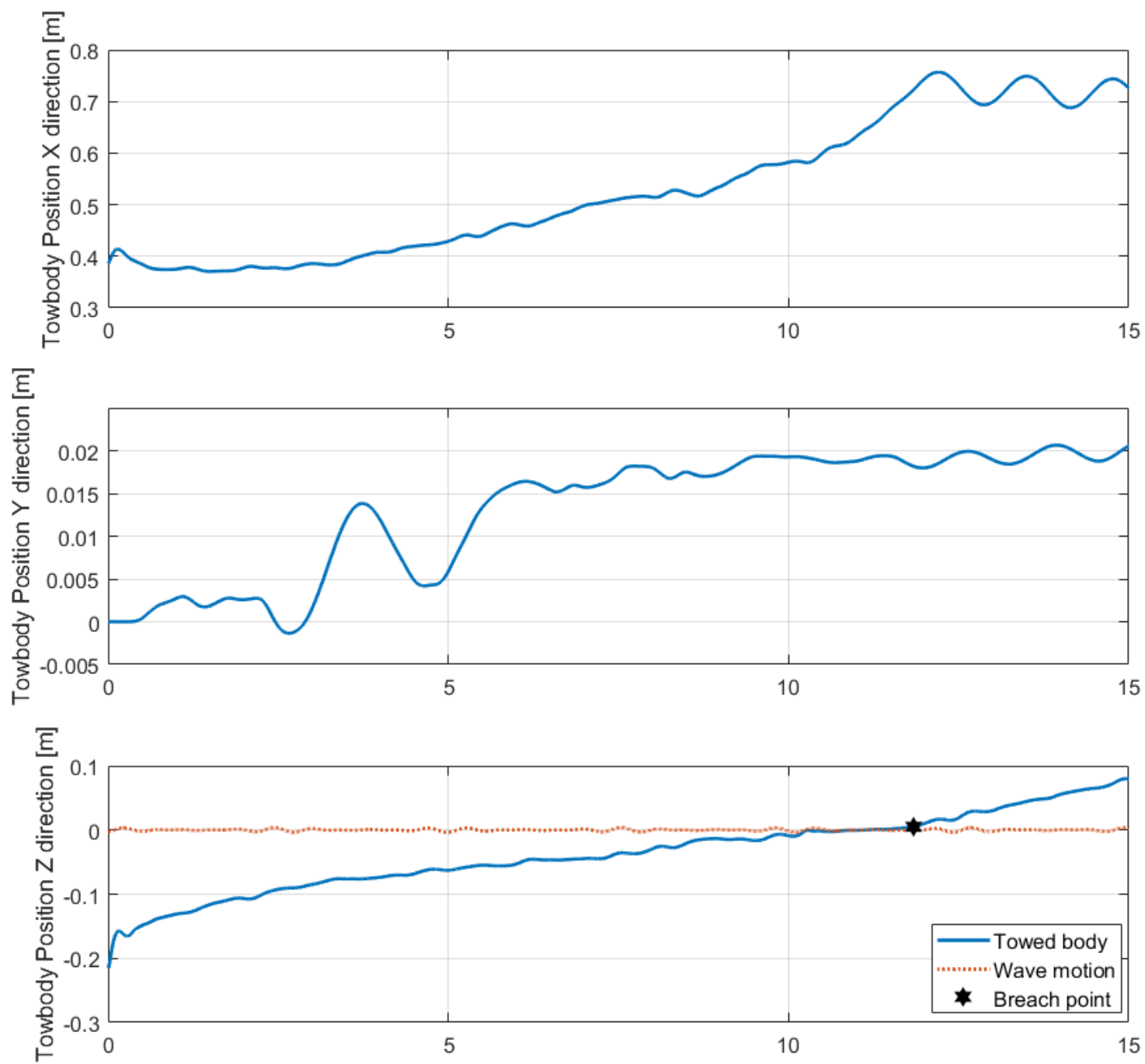

Figure 3.15: Simulated results for the towed body position with combined heave compensation and wave synchronization during a recovery operation with irregular waves at SS3. The towed body breach point is indicated by a hexagram.

\subsection{Summary}

Advances made to the computer simulator to study the effects of wave synchronization in a recovery operation contributes toward the first objective of this thesis. Both irregular and regular waves were modelled as well as the loads on the payload during breach. 


\section{CHAPTER 3. WAVE SYNCHRONIZATION MODEL AND SIMULATION}

The simulation results indicated that wave synchronization does not benefit compensator strategies based on the metrics used in the analysis for the scenario involving a towed body being lifted out of the water. When considering the standard deviation of the cable tension, the metric used by Sagutun et al., the simulations that were run with combined wave synchronization and heave compensation performed similarly to those run with only heave compensation. The large variations in tension observed in the simulations run with the combined wave synchronization and heave compensation are a concern for cable detachment, which is more likely to occur when the tension becomes suddenly small. Moreover, both the combined wave synchronization and heave compensation only strategies performed worse than simulations with no control method applied in all cases, which could suggest that the set-point algorithms presented by Calnan et al. [29] may not lend themselves to recovery operations when the body is lifted out of the water. It is suggested that other metrics be used to analyse the simulation results, such as the deviation of towed body motion from its trajectory, before rejecting the set-point algorithms for recovery operations.

In the next chapter, the use of a wave prediction algorithm for predicting safe breach conditions will be investigated. 


\section{Chapter 4}

\section{Advancing a Wave Prediction}

\section{Algorithm for Breach Condition Forecasts}

This chapter is based on a paper that was presented at the Canadian Society for Mechanical Engineering International Congress 2018 (CSME 2018) [37]. Additionally the work was used as part of an unmanned aerial vehicle landing study which was presented at the IEEE Oceans 18 conference [38].

In marine applications, the determination of the ship motion and the relative motion between two independent bodies is a challenging task. As previously discussed, the motions of both the host vessel and surface waves often define the availability for load transfer in launch and recovery operations. Chapter 3 explored the use of wave synchronization to achieve motion compensation during the breach phase, where recovery was attempted regardless of the severity of the relative motions between the ship and surface waves. Determining and anticipating safe breach conditions is presented as the next step in realizing successful recovery operations in any sea condition. To satisfy a safe breach condition, it is expected that knowledge of the 
CHAPTER 4. ADVANCING A WAVE PREDICTION ALGORITHM FOR

BREACH CONDITION FORECASTS

motions of the mothership and surface waves is required and that predicting these motions would be beneficial in anticipating the safe times to breach.

Sophisticated and established methods do exist that determine the motion of a ship in a seaway through panel or finite element methods [14, 39]; however, these methods do not lend themselves to real-time estimation. Moreover, these methods cannot be extrapolated to determine any generalized signal or motion. Kuchler et al. [40] presented an implementation of a wave-prediction algorithm, which combats transport delays between actual ship motion and measurement of ship motion. Woodacre et al. [3] was able to build upon Kuchler et al. [40] and use a version of the prediction algorithm as a previewing function for a Model-Predictive Control scheme (MPC). This chapter builds upon these works to generalize a Signal Prediction Algorithm (SPA) so that it can predict any periodic signal and presents the initial developments in defining the criteria required for a safe breach condition.

\subsection{Signal Prediction Algorithm}

The signal prediction method based on the work of Kuchler et al. [40] and Woodacre et al. $[3,41]$ is composed of three distinct parts: mode detection, estimation, and prediction. To predict the wave motion, the periodic components, or modes, must be identified. These modes are determined by decomposing the measured signal $s(t)$ into a set of $N$ sine waves expressed as

$$
s(t)=\sum_{i=1}^{N} A_{i} \sin \left(2 \pi f_{i} t+\epsilon_{i}\right)+v(t)
$$

where the amplitude $A$, frequency $f$, and phase $\epsilon$ of each mode $i$ are obtained by taking the Fast Fourier Transform (FFT) of past measured data over a specified time interval. Additionally, the static signal offset is denoted by $v(t)$. The specified observation window for the FFT, referred to as $T_{F F T}$, and sampling frequency are set 


\subsection{SIGNAL PREDICTION ALGORITHM}

depending on the frequency content of the known wave spectrum. The time window $T_{F F T}$ is chosen such that the lowest frequency of the wave spectrum can be detected, while also preventing aliasing. The sampling frequency of the FFT is selected such that a desired resolution in the frequency domain is achieved.

As conditions change, the number of modes $N$ and associated frequencies $f$ change with time; therefore, to predict the periodic motion, a peak detection algorithm is performed at every $T_{F F T}$ interval on the latest set of measured data. This peak detection algorithm determines the amplitude $A_{F F T}$ and frequency $f_{F F T}$ of each mode based on the dominant peaks in the FFT spectrum. A peak in the FFT spectrum is determined to be dominate when it exceeds a peak detection sensitivity $\mu$ which is set by the user. When a new set of modes is identified, it is used to initialize an observer model with a new set of parameters: $A_{F F T}, f_{F F T}, \epsilon_{F F T}$, and $N$. From the identified modes, the observer model is given as

$$
\dot{\mathbf{x}}=\left[\begin{array}{ccccc}
A_{1} & 0 & \ldots & \ldots & 0 \\
0 & A_{2} & \ddots & & \vdots \\
\vdots & \ddots & \ddots & \ddots & \vdots \\
\vdots & & \ddots & A_{N} & 0 \\
0 & \vdots & \vdots & 0 & 0
\end{array}\right]\left[\begin{array}{c}
x_{1} \\
x_{2} \\
\vdots \\
x_{N} \\
x_{o f f}
\end{array}\right], \quad s(t)=\left[\begin{array}{lllll}
C_{1} & C_{2} & \cdots & C_{N} & 1
\end{array}\right] \mathbf{x}
$$

where after each $T_{F F T}$ identification interval, $A_{i}$ is solved as

$$
A_{i}=\left[\begin{array}{cc}
0 & 1 \\
-\left(2 \pi f_{i}(t)\right)^{2} & 0
\end{array}\right] \text {. }
$$

Discretizing the continuous system model in Equation 4.2 yields for each time step $k$

$$
\mathbf{x}_{k+1}=\exp (\mathbf{A} \Delta T) \mathbf{x}_{k}, \quad s_{k}=\mathbf{C x}_{k}
$$

where the discrete system matrix $\boldsymbol{\Psi}$ is represented by the term $\exp (\mathbf{A} \Delta T)$. 
CHAPTER 4. ADVANCING A WAVE PREDICTION ALGORITHM FOR

BREACH CONDITION FORECASTS

The observer model is used to continuously estimate the mode parameters $A_{F F T}$ and $\epsilon_{F F T}$ such that the latest signal measurements are used to adapt $A_{F F T}$ and $\epsilon_{F F T}$ at each time step. For the prediction algorithm, a discrete Kalman filter is implemented to estimate the system states and has the form

$$
\hat{x}_{k+1}=\mathbf{\Psi} \hat{x}_{k}+\mathbf{L}\left(s_{k}-\hat{s}_{k}\right), \quad \hat{s}_{k}=\mathbf{C} \hat{x}_{k},
$$

where $k$ is the current time step, $\boldsymbol{\Psi}$ is the discrete system matrix, $\hat{\mathbf{x}}$ is the vector of observed states, $\mathbf{L}$ is the observer gains matrix, $\hat{s}$ and $s$ are the estimated motion and measured motion respectively, and $\mathbf{C}$ is the system output matrix. The discrete system matrix, observed states and system output matrix were derived following the work of Kuchler et al. [3]. The observer gain matrix is obtained by the solution to the discrete algebraic Riccati equation.

From each detected mode, two observer states $x_{i, 1}$ and $x_{i, 2}$ are estimated and then rearranged to solve for the adapted observer parameters $\epsilon_{o b s}$ and $A_{o b s}$ at the current time $t_{k}$, such that:

$$
\begin{array}{r}
\epsilon_{o b s, i, k}=\arctan \left(\frac{2 \pi f_{i} \hat{x}_{i, 1}}{\hat{x}_{i, 2}}\right)-2 \pi f_{i} t_{k} \\
A_{o b s, i, k}=\frac{\hat{x}_{i, 1}}{\sin \left(2 \pi f_{i} t_{k}+\epsilon_{o b s}\right)} .
\end{array}
$$

Finally, the prediction algorithm can be used to forecast the motion sequence over a prediction horizon $T_{\text {Pred }}$, i.e. at the time $t_{k}+T_{\text {Pred }}$. Referring to Equation 4.1, the predicted motion at $t_{k}+T_{\text {Pred }}$ is

$$
s_{\text {Pred }}\left(t_{k}+T_{\text {Pred }}\right)=\sum_{i=1}^{N} A_{o b s, i, k} \sin \left(2 \pi f_{i}\left(t_{k}+T_{\text {Pred }}\right)+\epsilon_{o b s, i, k}\right)+v(t) .
$$

For the purposes of this work, the SPA is used to determine a Go-NoGo command based on given GO criteria. The GO criteria could be one or several parameters used to define the acceptable breach conditions for a GO scenario. The 


\subsection{BENCHMARK DATA}

criteria are compared with the predicted signal over the continuous prediction horizon $T_{\text {Pred }}$ at each time step. If over the prediction horizon the predicted signal meets the GO criteria, a GO command is output. Conversely, if the GO criteria are not met by the predicted signal over the prediction horizon, a NoGo command is output. Figure 4.1 summarizes the structure of the prediction method and Go-NoGo discriminator.

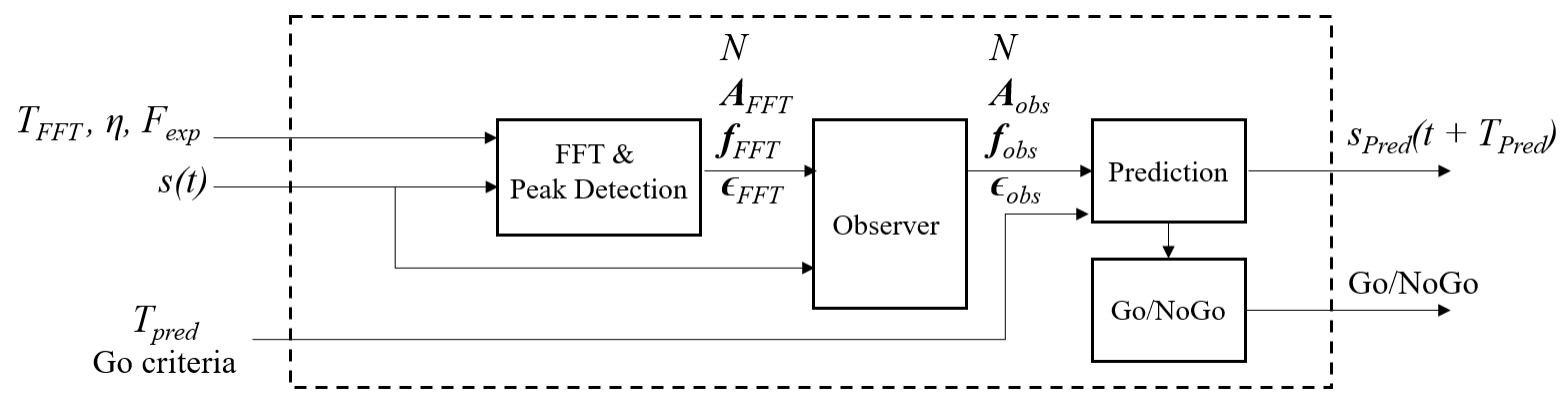

Figure 4.1: Structure overview of the prediction method and Go-NoGo discriminator.

\subsection{Benchmark Data}

Figure 4.2 shows the benchmark test cases used as the input signal $s(t)$ throughout this chapter. The test case originates from a set of digitized ship motion data from the Australian Defence Science and Technology Organisation (DSTO) report [19]. The signals $s(t)$ are the resolved 6-DOF ship motion of the data, in metres and degrees.

\subsection{Mode Detection Tuning}

In the SPA formulation section above, it was discussed that to detect the dominant modes of the incoming signal it is necessary to select an appropriate FFT observation window $T_{F F T}$ and peak detection sensitivity $\mu$. The selection of an inappropriate $T_{F F T}$ may result in two undesirable scenarios: the first being too short an interval that results in no modes detected, and the second being too long an interval that results in modes detected at a rate that does not keep up with changing conditions, 

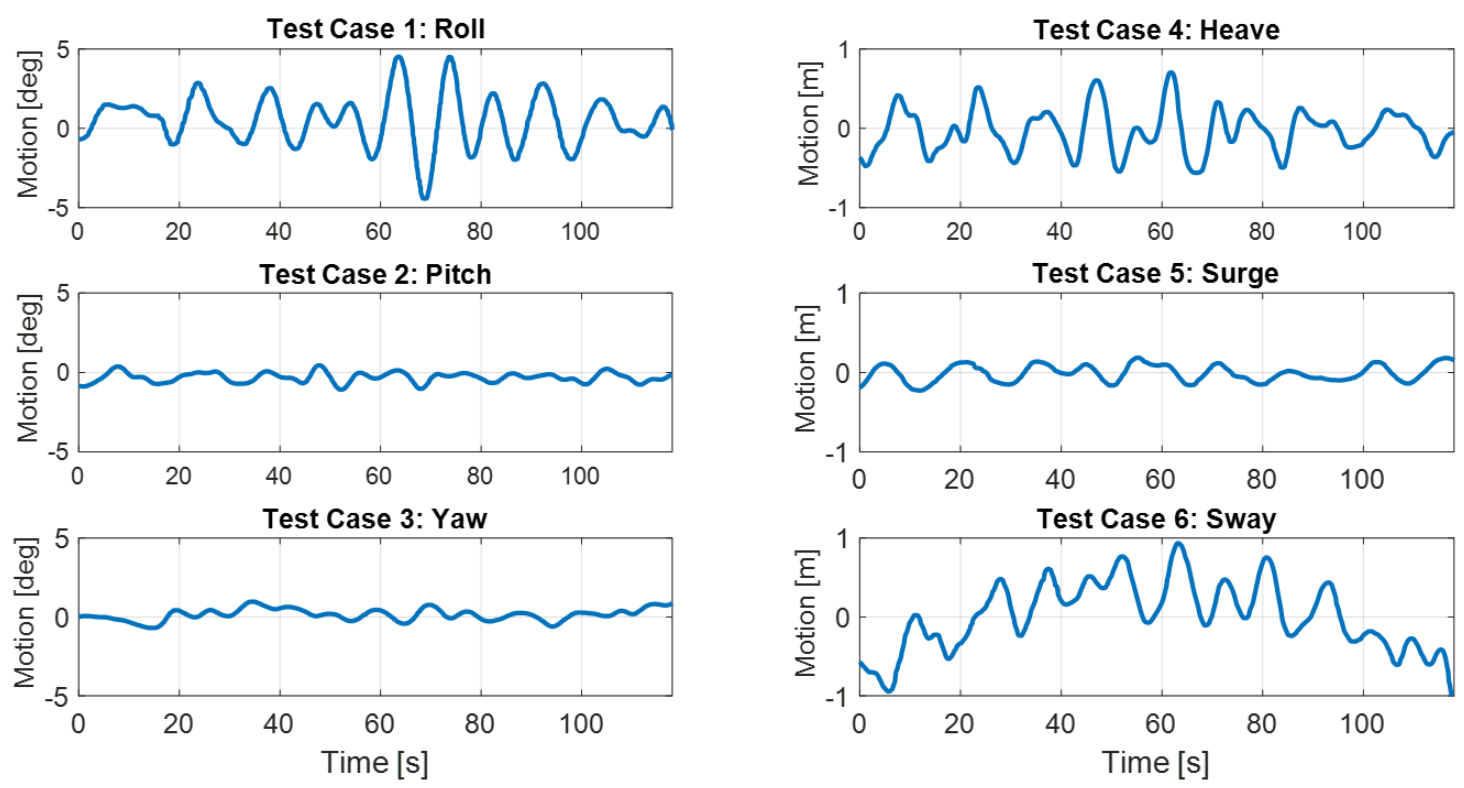

Figure 4.2: Benchmark test cases of resolved ship motion from the Australian DSTO report.

which could yield aliasing. To examine these issues, Figure 4.3 plots the roll motion input signal $s(t)$ from Figure 4.2, as a solid blue line, and the prediction $s_{\text {Pred }}(t)$, as a dashed red line, at $0.25 \mathrm{~s}$ into the future. $s_{\text {Pred }}(t)$ is shifted back 0.25 seconds, i.e. 25 time steps, to place both the input signal and the prediction in the same time frame. The dotted green line between $70 \mathrm{~s}$ and $80 \mathrm{~s}$ in Figure 4.3 depicts the scenario where there is no predicted signal due to no modes detected during the previous FFT observation window.

To address the issue of discontinuities in mode detection, the detection algorithm was amended such that in the case where zero modes are detected, FFT observation interval is expanded every time step until at least one new mode is detected. The next mode detection is then performed after another $T_{F F T}$ interval, starting where the new set of modes was detected. In this way, the mode detection algorithm can handle slower than expected modes that may exist in the signal $s(t)$. In addition to the latter, the proposed amendments stated above also helps with avoiding the second undesirable scenario, as one can set a shorter $T_{F F T}$ to avoid aliasing, without needing to consider the $T_{F F T}$ length required to ensure continuous mode 


\subsection{MODE DETECTION TUNING}

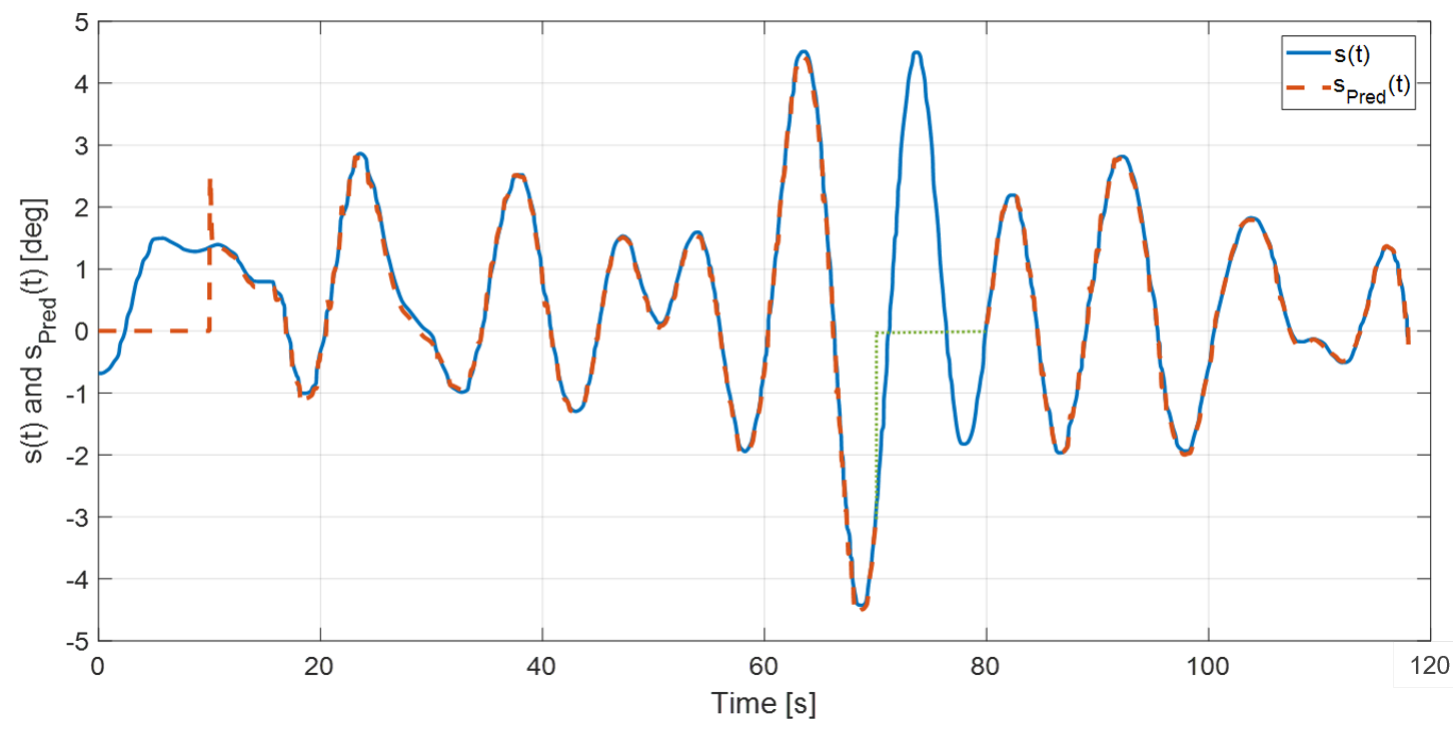

Figure 4.3: Measured and predicted signal together, with prediction signal time shifted to the input signal timeframe. The prediction discontinuity due to no modes detected is shown in green.

detection. However, it is important that $T_{F F T}$ is selected such that the minimum frequency of the input signal $f_{\min }$ is discoverable. $T_{F F T}$ sets the frequency resolution, $d f$ of the Fourier spectrum analysis with the minimum discoverable frequency given as

$$
f_{\min }=d f=\frac{1}{T_{F F T}} .
$$

Equation 4.8 shows how setting a shorter $T_{F F T}$ window results in a coarser frequency resolution [42]. Maximum detectable frequency is determined by the Nyquist frequency $f_{N}=1 /(2 d t)$.

The peak sensitivity $\mu$ describes the minimum peak height; a threshold value used by the peak detection algorithm to determine the dominant peaks in the FFT spectrum. Figure 4.4 shows the amplitude-frequency spectrum of the first $10 \mathrm{~s}$ of the benchmark test case with $\mu=0.01$, indicated by a dashed red line. For this $\mu$, the number of peaks detected is 6 , denoted by the triangle markers. By inspection of the first $10 \mathrm{~s}$ of the benchmark test case for roll motion in Figure 4.2, to capture the dominate motion at most two peaks should be detected. Any other peaks in the FFT 
CHAPTER 4. ADVANCING A WAVE PREDICTION ALGORITHM FOR

BREACH CONDITION FORECASTS

spectrum is attributed to sensor noise. By increasing the value of $\mu$, the sensor noise can be rejected. Shown as a dashed red line in Figure 4.5, $\mu$ has been increased by a factor of $10(\mu=0.1)$ and the number of peaks detected is two. The selection of peak detection sensitivity $\mu$ must therefore be set based on sensor noise and is case specific. Future work could examine a recursive methodology to determine if a peak is valid or estimate how many peaks should be identified.

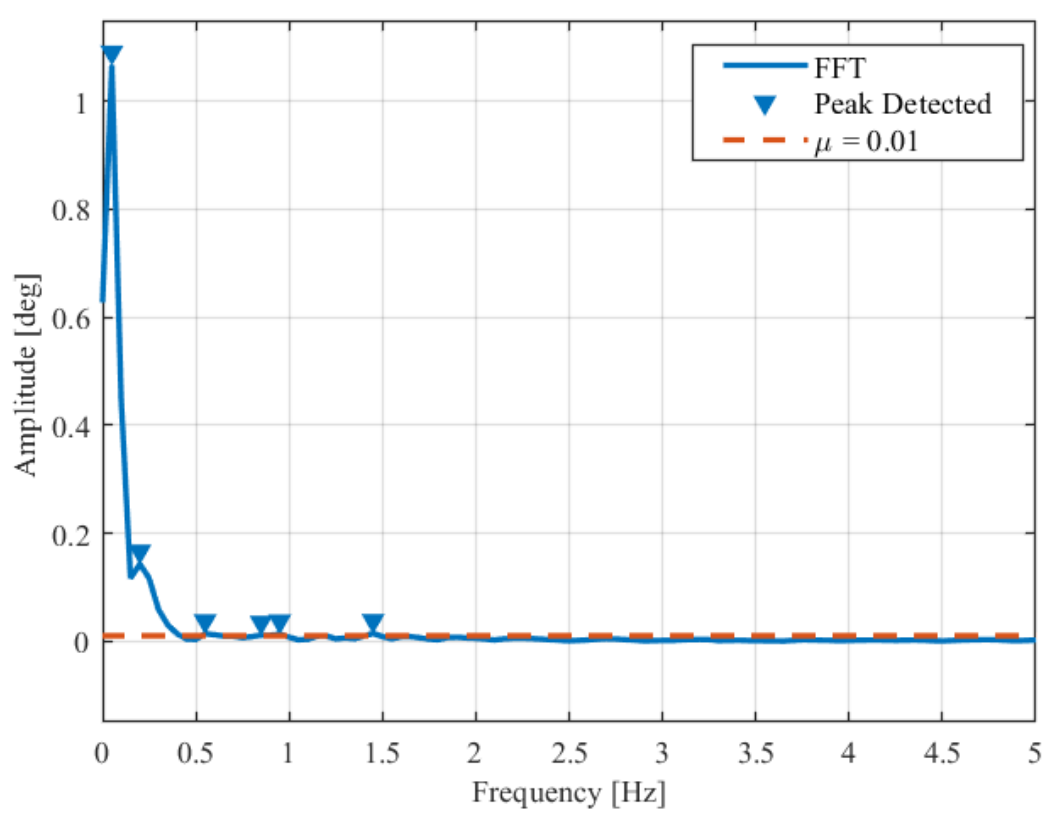

Figure 4.4: Dominant peaks detected from the FFT spectrum of the first $10 \mathrm{~s}$ of the benchmark case for $\mu=0.01$.

\subsection{Prediction Algorithm Simulation Results}

The SPA detailed in Sections 4.1 and 4.3 was implemented in MATLAB and simulated in the Simulink environment. Figure 4.6 depicts the Simulink block that calls on the SPA function. The input variables include input signal $s(t)$; the GO criteria; tuning parameters $T_{F F T}$ and $\mu$; and the prediction horizon $T_{\text {Pred }}$. The SPA block outputs the predicted signal $s_{\text {Pred }}\left(t+T_{\text {Pred }}\right)$ and the Go/NoGo command signal. A fixed step, discrete solver was used with fixed step $d t=0.01 \mathrm{~s}$. For the benchmark roll motion 


\subsection{PREDICTION ALGORITHM SIMULATION RESULTS}

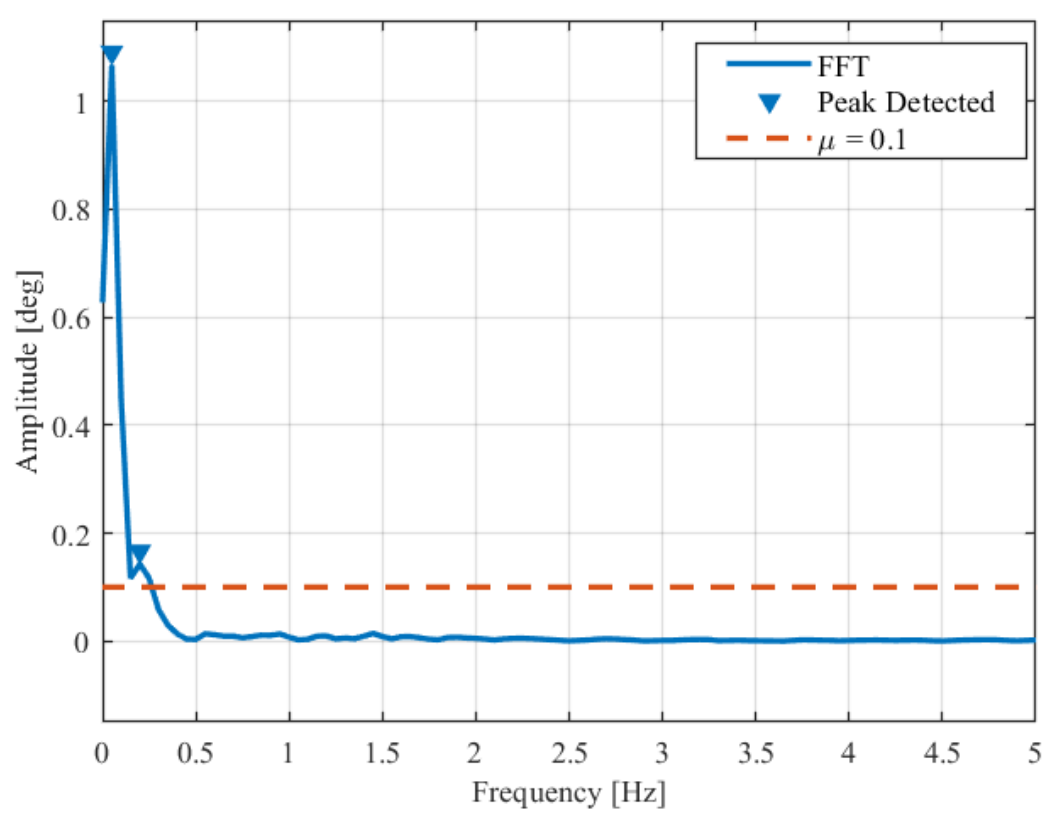

Figure 4.5: Dominant peaks detected from the FFT spectrum of the first $10 \mathrm{~s}$ of the benchmark case for $\mu=0.1$

case in Figure 4.2, the simulation runtime was approximately $11 \mathrm{~s}$ for the full $118 \mathrm{~s}$ of the benchmark signal.

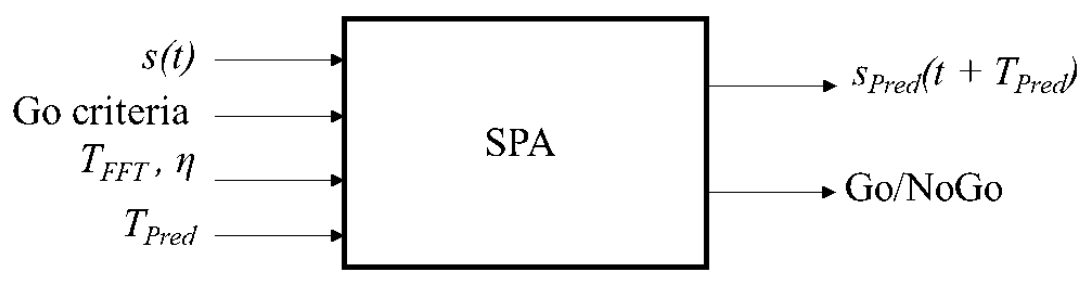

Figure 4.6: SPA function block implemented in the Simulink environment.

Figure 4.7 displays the predicted signal, solid red line, over a continuous prediction horizon of $10 \mathrm{~s}$ for the benchmark input signal, solid blue line, at time $t$ $=38 \mathrm{~s}$. The parameters used for the prediction were: $T_{F F T}=10 \mathrm{~s}$ and $\mu=0.1$. In Figure 4.7, good accordance is observed between the input signal and predicted curve until $T_{\text {Pred }}=3 \mathrm{~s}$. To investigate this further, the predicted signal is examined for $1 \mathrm{~s}, 3 \mathrm{~s}$ and $10 \mathrm{~s}$ prediction horizons. Figure 4.8a depicts the prediction error for the prediction at $1 \mathrm{~s}$ into the future at every point in time. Similarly, Figure $4.8 \mathrm{~b}$ and $\mathrm{c}$ respectively depict the prediction error at $3 \mathrm{~s}$ and $10 \mathrm{~s}$ into the future at every 
CHAPTER 4. ADVANCING A WAVE PREDICTION ALGORITHM FOR

BREACH CONDITION FORECASTS

point in time. Note that the first $11 \mathrm{~s}$ are omitted to ignore the initialization period of the SPA. The maximum, minimum, mean and standard deviation of the error for each case are tabulated in Table 4.1 for $t=11 \mathrm{~s}$ to $110 \mathrm{~s}$. From Table 4.1, both the mean and standard deviation of the absolute error are significantly larger, by an order of magnitude, for the longest prediction horizon of $10 \mathrm{~s}$. The latter is consistent with what was qualitatively observed in Figure 4.7 at $t=38 \mathrm{~s}$. The results in Table 4.1 suggest that determining the likelihood of success of a process to produce a GoNoGo command becomes less accurate for longer prediction horizons as the mean error increases significantly over this time interval. Furthermore, defining the GO scenario based on the maximum error observed over $T_{\text {Pred }}$ alone is not representative of true future events for longer predictions. The following section will develop useful GO criteria that considers the error that exists in the prediction.

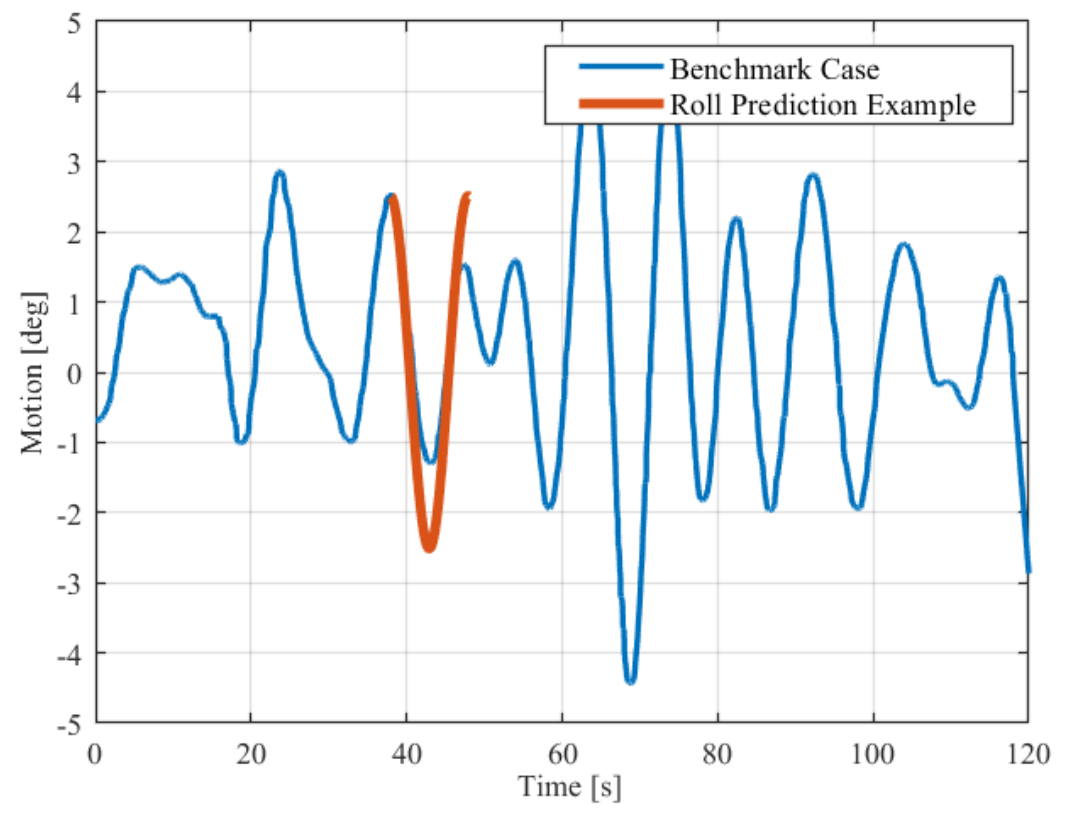

Figure 4.7: Benchmark data with prediction curve at $t=38$ s plotted over a continuous time horizon of $10 \mathrm{~s}$ into the future. 


\subsection{DEVELOPING THE GO/NOGO CRITERIA}
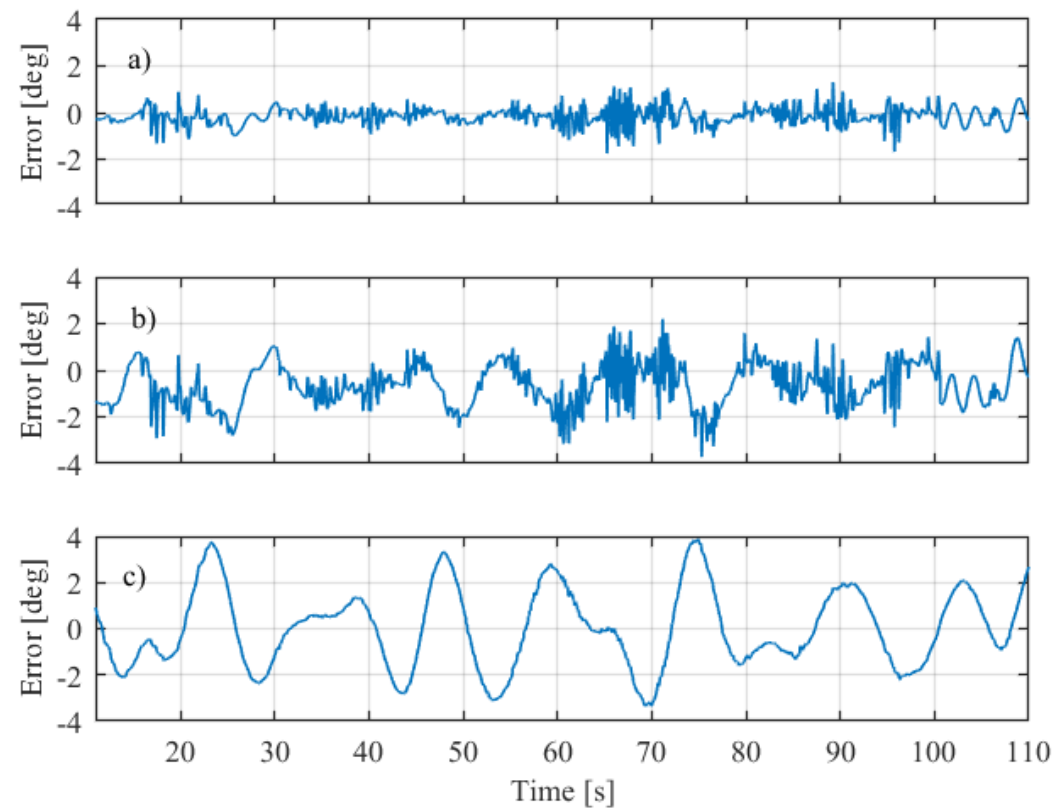

Figure 4.8: Error between benchmark data and prediction at a) $1 \mathrm{~s}$ into the future, b) $3 \mathrm{~s}$ into the future and c) $10 \mathrm{~s}$ into the future.

\begin{tabular}{|l|l|l|l|}
\hline \multirow{2}{*}{ Error $[\mathbf{d e g}]$} & \multicolumn{3}{|l|}{ Prediction Horizon, $\mathbf{T}_{\text {Pred }}$} \\
\cline { 2 - 4 } & $\mathbf{T}_{\text {Pred }}=\mathbf{1} \mathbf{~}$ & $\mathbf{T}_{\text {Pred }}=\mathbf{3} \mathbf{~}$ & $\mathbf{T}_{\text {Pred }}=\mathbf{1 0} \mathbf{~}$ \\
\hline Maximum & 1.74 & 3.72 & 3.88 \\
\hline Minimum & $4.57 \times 10^{-6}$ & $4.41 \times 10^{-4}$ & $2.93 \times 10^{-4}$ \\
\hline Mean & 0.29 & 0.89 & 1.41 \\
\hline Standard Deviation & 0.35 & 0.92 & 1.69 \\
\hline
\end{tabular}

Table 4.1: Error metrics for various prediction horizons.

\subsection{Developing the Go/NoGo Criteria}

The following section investigates the development of useful definitions of the GO scenario, to be identified based on the GO criteria.

The first definition of the GO scenario to be investigated is the simple Maximum Prediction Threshold (MPT) approach. This approach identifies a GO scenario if over the entire prediction horizon, the maximum value of the predicted signal is below the given threshold value. In other words, only one point of the predicted signal must to be above the threshold to output a NoGo command. The results in 
CHAPTER 4. ADVANCING A WAVE PREDICTION ALGORITHM FOR

BREACH CONDITION FORECASTS

Table 4.1 suggest that this simple Maximum Prediction Threshold approach would not provide a useful definition of the GO scenario for longer $T_{\text {Pred }}$, as there exists larger error with the predicted signal as the prediction horizon increases. Two more GO criteria are suggested below that are defined such that the error that exists in the prediction is considered.

If instead, the GO scenario is defined based on the statistical spread of the predicted signal, it is possible to relax how the GO criteria identify a GO scenario. The spread of 1 Standard Deviation (1-SD) approach outputs a GO command when the prediction mean, evaluated over the prediction horizon, plus 1 standard deviation of the prediction is below a threshold value. The 1-SD approach is expressed empirically in Equation 4.9 as

$$
\text { Go Criteria }>\overline{s_{P r e d, k}}+\sigma_{k},
$$

where $\overline{S_{\text {Pred,k }}}$ is the prediction mean over $T_{\text {Pred }}$ at time step $k$, and $\sigma$ is the standard deviation of the prediction over $T_{\text {Pred }}$ at time step $k$. Equation 4.9 is equivalent to stating that for any randomly selected sample of the predicted signal over $T_{\text {Pred }}$, a GO command is output if $68 \%$ of the sampled points fall below the threshold.

The spread of 1.645 Standard Deviations (1.645-SD) approach defines the GO scenario to occur when the prediction mean plus 1.645 standard deviations is below the threshold value. The 1.645-SD approach is expressed empirically in Equation 4.10 as

$$
\text { Go Criteria }>\overline{s_{\text {Pred,k }}}+1.645 \sigma_{k} .
$$

Equation 4.10 is equivalent to stating that for any randomly selected sample of the predicted signal over $T_{\text {Pred }}$, a GO command is output if $90 \%$ of the sampled points fall below the threshold. Both methods described in Equations 4.9 and 4.10 are based on the same principle but differ in how conservatively they evaluate the predicted signal.

To evaluate the three definitions of the GO scenario MPT, 1-SD and 1.645$\mathrm{SD}$, the SPA was run for each case with the benchmark roll motion data and com- 


\subsection{DEVELOPING THE GO/NOGO CRITERIA}

pared with the desired Go-NoGo command signal. The desired command signal was determined by setting the command as GO when the benchmark case is below the threshold value and setting the command as NoGo when the benchmark case is above the threshold value. Figure 4.9a shows the input signal, left axis, as a solid line with the GO criteria threshold, set as $2.5^{\circ}$, as a dashed line. In Figure $4.9 \mathrm{a}$, the desired Go-NoGo command signal, right axis, is also shown for $t=50 \mathrm{~s}$ to $t=90 \mathrm{~s}$ of the simulation. Here $\mathrm{GO}=1$ and $\mathrm{NoGo}=0$. Figure 4.9 part b, c and d depict the Go-NoGo command signal for the MPT, 1-SD and 1.645-SD approaches respectively. The three GO scenario definitions were evaluated based on the number of seconds that a GO command is output compared to the amount of GO time registered by the desired command signal. Asbefore, when obtaining the GO time, the first $11 \mathrm{~s}$ of data are omitted to ignore the SPA initialization period. In Table 4.2, the GO time for the benchmark case from $t=11 \mathrm{~s}$ to $t=118 \mathrm{~s}$ is tabulated for each approach.
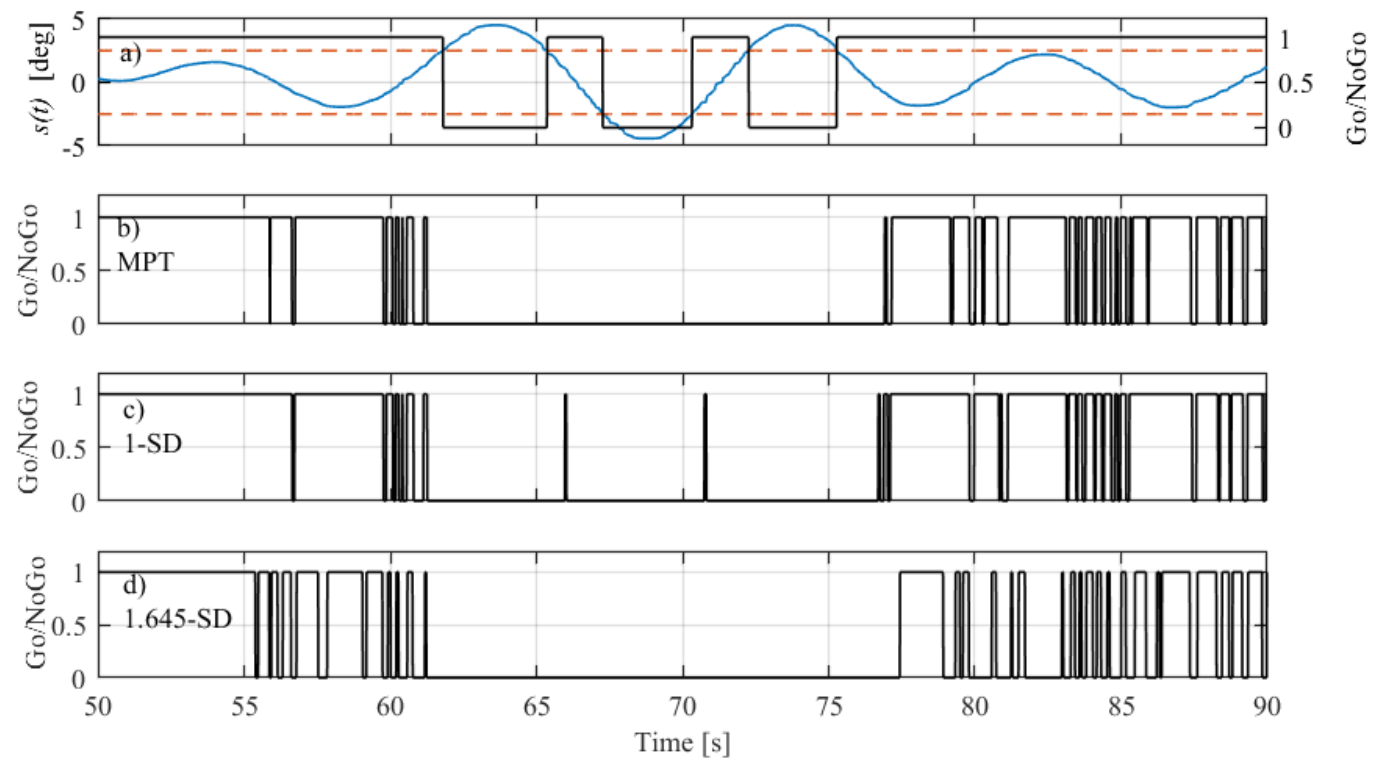

Figure 4.9: Go-NoGo command signals with desired command signal in a), and resulting command outputs for approaches b) MPT, c) 1-SD and d) 1.645-SD. For display purposes, the results are shown for $t=50 \mathrm{~s}$ to $t=90 \mathrm{~s}$.

Simulations were also run for the other five benchmark test cases in Figure 4.2 where the results of each approach are summarized for all benchmark test cases 
CHAPTER 4. ADVANCING A WAVE PREDICTION ALGORITHM FOR BREACH CONDITION FORECASTS

\begin{tabular}{|c|c|c|c|c|}
\hline \multirow{2}{*}{ "GO" metric } & \multicolumn{4}{|c|}{ "GO" scenario definition $[11 \mathrm{~s}-118 \mathrm{~s}]$} \\
\hline & Desired & MPT & 1-SD & 1.645-SD \\
\hline "GO" time $[\mathrm{s}]$ & \multirow{2}{*}{98.98} & 87.71 & 94.78 & 77.94 \\
\hline Error $[\mathrm{s}]$ & & 11.27 & 4.20 & 21.04 \\
\hline
\end{tabular}

Table 4.2: Evaluation of GO scenario definitions.

in Figure 4.10. Figure 4.10 plots the "GO time" error between the desired amount of time a GO state should be output, based on the input signal, and the actual mount of tune a GO state is output from the predicted signal. The results of the simulations were in accordance with what was observed in Figure 4.9 where, over a 10 s prediction horizon, the 1-SD approach best matched the desired Go-NoGo signal for five of six test cases. In test case 5 the surge motion was below the threshold value of $0.5 \mathrm{~m}$ over the entire simulation therefore both the input and predicted signal output a GO state over the entire simulation time. Hence no error was observed in the "GO time".

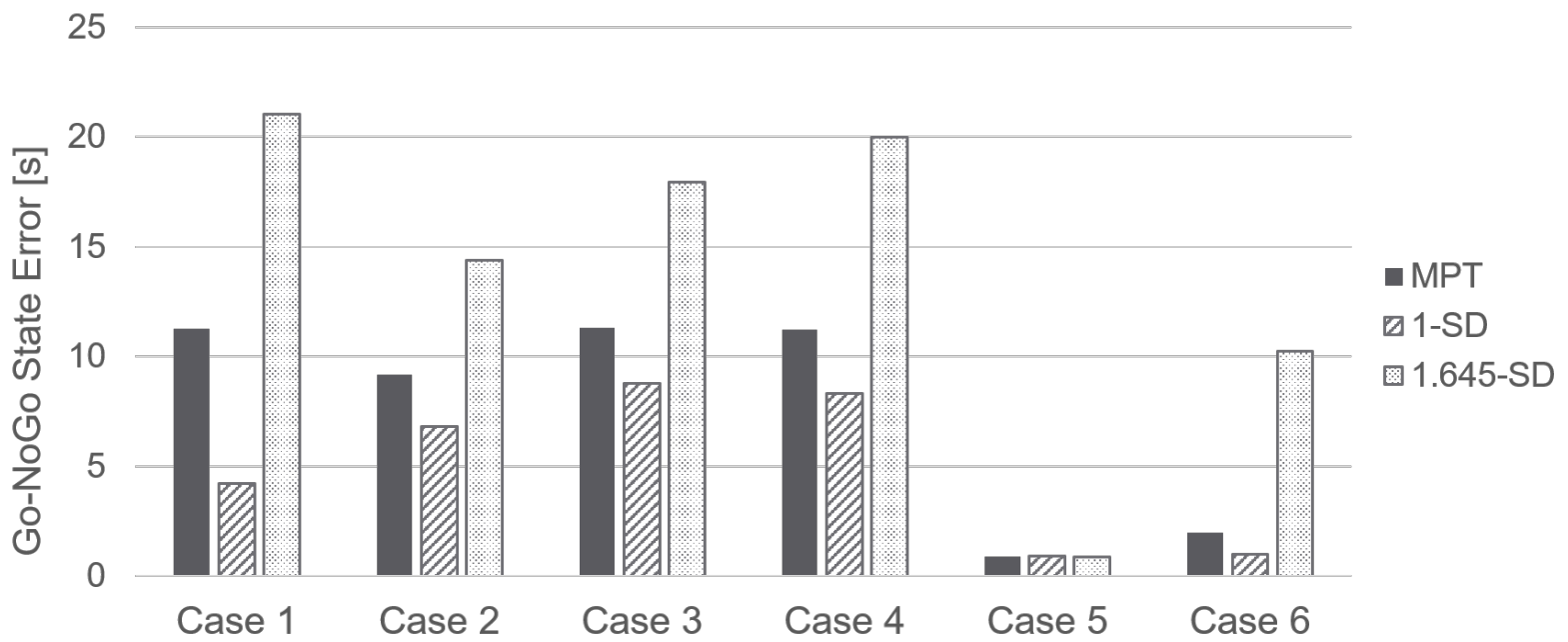

Figure 4.10: Summary of "Go time" error between desired amount of time a GO state should be output and the actual amount of time a GO state is output for all six benchmark test cases.

\subsection{Go-NoGo Command Latching}

Observing the Go-NoGo signals in Figure 4.9b, c and d at $60 \mathrm{~s}, 80 \mathrm{~s}$ and $90 \mathrm{~s}$, there is rapid fluctuation in the command signal. For a physical implementation of the 


\subsection{GO-NOGO COMMAND LATCHING}

SPA-Go-NoGo function lifting operations, it is desired that the SPA latches on to a GO or NoGo command to meet the responsivity of the handling equipment system components. A method for Go-NoGo command latching is proposed below to limit this undesirable fluctuation.

Figure 4.11 shows the logic flowchart for the latching algorithm. To start, the latching algorithm receives the Go-NoGo state determined from the predicted signal. Next, the algorithm looks to see if a target state has been set. If no target state has been set, the current Go-NoGo state is set as the target state. The algorithm must then determine whether it will commit to this target by watching the incoming Go-NoGo state over an evaluation period $T_{\text {eval }}$. During this evaluation period, if at any point the incoming Go-NoGo state differs from the target state, the current GoNoGo state is set as a new target state and $T_{\text {eval }}$ is reset. However, if all Go-NoGo states received during the evaluation period match the target state, the algorithm will "latch" onto the target state and output this command over the runtime interval $T_{\text {run }}$. After $T_{\text {run }}$ is complete, a new evaluation period begins.

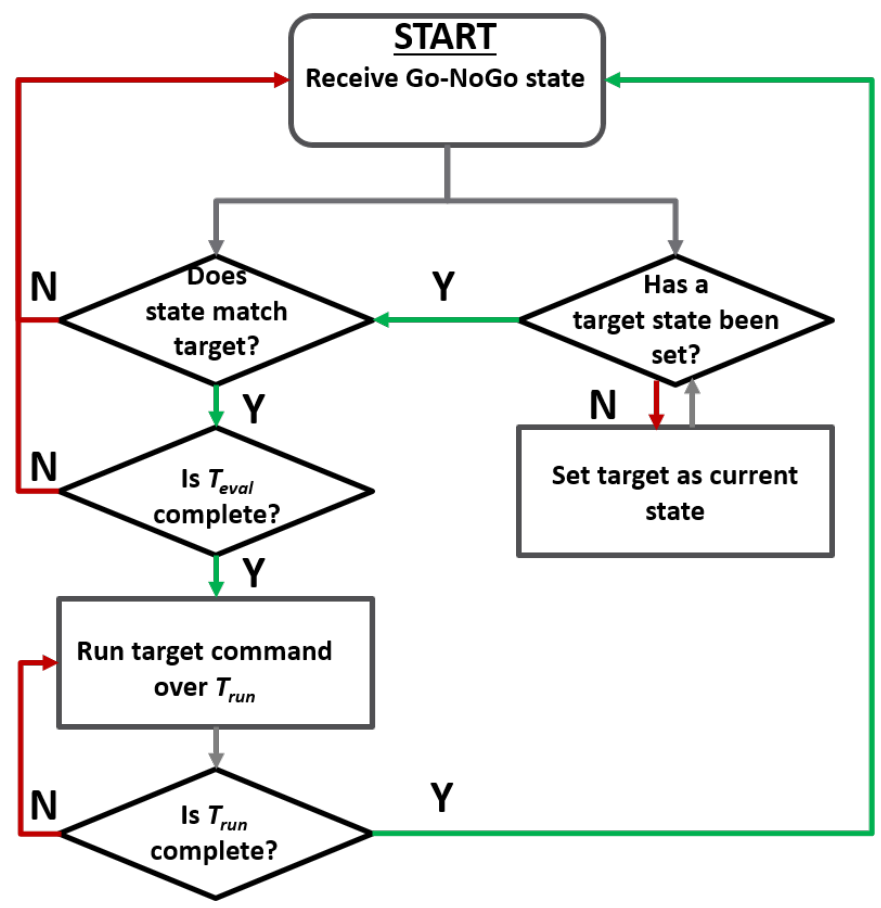

Figure 4.11: Flow chart of the logic used in the command signal latching algorithm. 
CHAPTER 4. ADVANCING A WAVE PREDICTION ALGORITHM FOR

BREACH CONDITION FORECASTS

Figure 4.12 shows the results of the latching algorithm where the Go-NoGo command signal without latching is shown in 4.12a, and the Go-NoGo command signal with latching is shown in $4.12 \mathrm{~b}$. For illustrative purposes, $T_{\text {eval }}$ was set as 0.1 $\mathrm{s}$ and $T_{\text {run }}$ was set as $2 \mathrm{~s}$, i.e. if a GO condition is sustained for $0.1 \mathrm{~s}$ the system will latch in a GO condition for at least 2 seconds. The command signals are output for the benchmark roll motion test case for $t=50 \mathrm{~s}$ to $t=90 \mathrm{~s}$. It is observed that for the selected $T_{\text {eval }}$ and $T_{\text {run }}$, all fluctuation was removed from the original command signal in Figure 10 a. $T_{\text {eval }}$ and $T_{\text {run }}$ are case specific and should be based on the physical handling equipment involved.
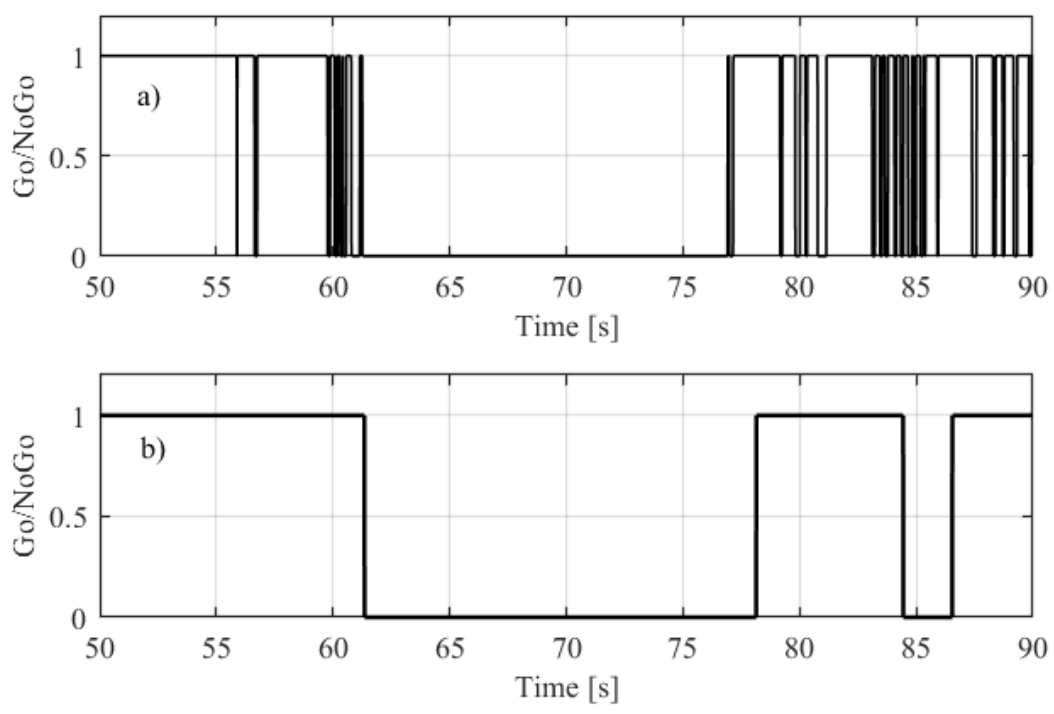

Figure 4.12: Go-NoGo command signals for the benchmark test case without latching in a) and with command latching in b). For display purposes, the results are shown for $t=50 \mathrm{~s}$ to $t=90 \mathrm{~s}$.

\subsection{Summary}

This chapter described the generalized formulation and tuning of a signal prediction algorithm as it applies to the determination of a Go-NoGo state for predicting safe breach conditions. A key modification made to the SPA was the implementation of 


\subsection{SUMMARY}

continuous mode detection that enables the algorithm to dynamically handle changing conditions of the input signal. Tuning the SPA also included setting a peak height sensitivity parameter that is used for determining the dominant peaks in the FFT spectrum during mode detection. By investigating the FFT spectrum of one identification period of the input signal, it was determined that the peak height sensitivity should be set such that it ignores sensor noise in the measured signal and is therefore case specific. For the normalized amplitudes of both wave and ship motion, setting the peak sensitivity parameter to a value of $\mu=0.1$ was judged to be sufficient in simulations.

The chapter also described the development of useful GO criteria used for producing the Go-NoGo state used to identify safe breaching opportunities. Because prediction error increases over the prediction horizon, it was determined that a GO scenario identified by the maximum point of the prediction should not be used, as this approach can lead to erroneous conclusions regarding the Go-NoGo state. Instead, a GO scenario defined by the mean and one standard deviation of the predicted signal over the prediction horizon was found to produce a Go-NoGo signal that agreed most with the desired Go-NoGo signal. Performance of the SPA-Go-NoGo function was further improved with the implementation of a latching algorithm that successfully removed undesirable fluctuation in the Go-NoGo signal.

Abujoub et al. [38] performed a study on unmanned aerial vehicle landings on maritime vessels that used simulated laser ranging and detecting devices (LIDAR) in conjunction with the signal prediction algorithm (SPA) presented in this chapter to forecast when a vessel's motion was within safe landing limits. Their results showed that with the use of the SPA, the number of UAV landing attempts was decreased by an average of 2 attempts, per test case, when compared to a system that did not use an SPA. It is anticipated that marine launch and recovery operations of towed bodies can also benefit from the proposed methods of determining a Go-NoGo state. Specifically, with the breach phase of recovery operations, the SPA can be employed to estimate the safe breach condition, where the motion of the ship and sea are below 
CHAPTER 4. ADVANCING A WAVE PREDICTION ALGORITHM FOR BREACH CONDITION FORECASTS

critical threshold values. The threshold values that determine safe conditions will be investigated in future work. From the estimated safe conditions, a Go-NoGo state will be inferred that can be used to increase the effectiveness of lifting a towed body from the sea. 


\section{Chapter 5}

\section{Development and Quantification of a Wave Generator and Ship Motion Simulator}

Chapter 2 introduced the design specifications for the equipment required to emulate wave motion and ship motion in the flume tank laboratory. In this chapter, the detailed designs of a plunger-type wavemaker and a robotic ship motion simulator will be presented along with the theoretical analyses conducted to quantify both systems. Section 5.1 presents a numerical analysis of small amplitude waves generated by a

plunger-type wave maker and includes an extension of this theory to waves propagated by a current. Section 5.2 suggests a design concept for the plunger-type wavemaker that was selected in Chapter 2 and discusses important design considerations for actuation and wedge shape. Section 5.3 introduces the design components of the robotic ship motion simulator followed by a kinematic analysis and discussion of the control scheme in Section 5.4. Section 5.5 presents preliminary experimental validation of the ship motion simulator conducted in the flume tank laboratory. Finally, Section 5.6 summarizes the designs of both the wavemaker and ship motion simulator and provides recommendations for building the wavemaker. 
CHAPTER 5. DEVELOPMENT AND QUANTIFICATION OF A WAVE

GENERATOR AND SHIP MOTION SIMULATOR

\subsection{Theoretical analysis of a plunger-type wave- maker}

Wavemaker theory is used to establish the relationship between wave amplitude and wavemaker stroke length. Early wavemaker theory was developed by Havelock [43], who proposed the first general theory for wave generation on a water surface. A simplified theory was proposed by Galvin [44] for shallow water waves where it was inferred that the volume of water displaced by a wavemaker should be equal to the crest volume of the propagating wave. Wavemaker investigations have primarily focused on studies of piston-type and flap-type wavemakers, which follows directly from the boundary value problem for linear wave theory presented in section 2.3.1, where a kinematic boundary condition replaces the lateral boundary condition at the piston surface [9].

Plunger-type wavemakers are theoretically more complex than either piston or flap type wavemakers. While a complete analytic solution for plunger-type wavemakers does not exist, several semi-analytic solutions to the plunger-type wavemaker problem have been investigated. Ursell [45] explored waves generated by a vertically oscillating circular cylinder where a boundary condition was imposed on the surface of the cylinder such that the fluid velocity normal to the cylinder surface was equal to the normal velocity component of the cylinder. Wang [23] proposed a solution for a more general wedge shaped plunger via a two parameter conformal transformation. Wang's solution was based on the deep water assumption and does not provide a means to study the effects of water depth. Wu [24] investigated small-amplitude waves generated by a vertically oscillating wedge in a finite depth wave tank using the boundary collocation method in order to investigate the effects of water depth in wave generation. The next section derives the relationship between wave amplitude and wavemaker stroke length for a plunger-type wavemaker. 


\subsubsection{Plunger-type wavemaker boundary value problem}

Figure 5.1 depicts the schematic of the plunger-type wavemaker problem for a wedge shaped plunger with a triangular cross-section described by a mean submerged width $b$ and a mean submerged depth $d$ both measured at still water level. $\beta$ is the wedge angle measured from the vertical $z$ axis. The plunger is actuated to oscillate vertically at frequency $\omega$ with stroke amplitude $S$ in order to generate surface waves that propagate in the positive $x$ direction in a water channel of constant depth $h$. The clearance between the channel bottom and wedge bottom is $d^{\prime}$ and $\overrightarrow{\mathbf{n}}$ represents the vector normal to the surface of the wedge. Similar to wave theory presented in Chapter 2, $\eta(x, t)$ represents the position of the free surface at position $x$ and at time $t$ relative to the plane $z=h$.

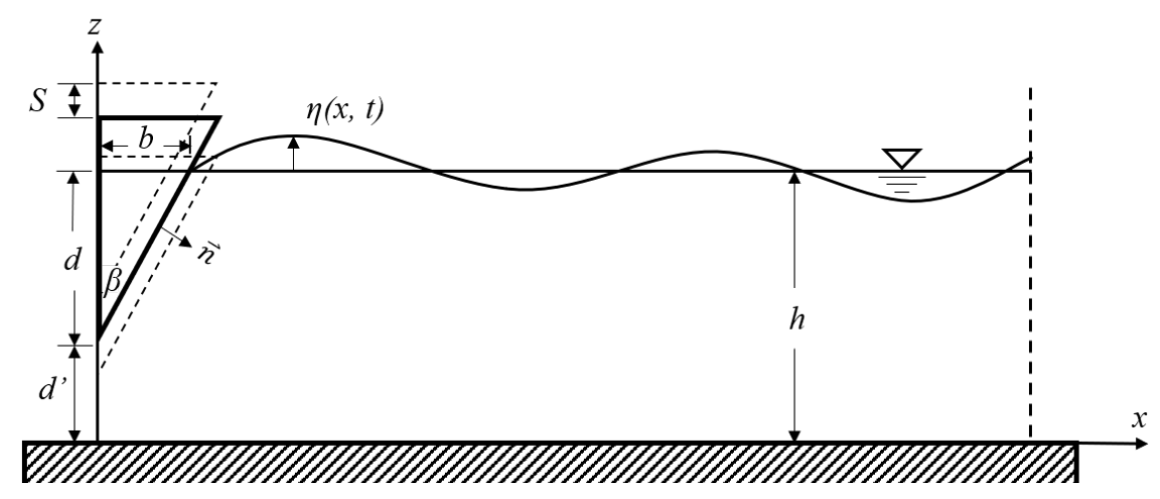

Figure 5.1: Schematic of the plunger-type wavemaker problem.

Under the same assumptions made in section 2.3.1 of irrotational and incompressible flow, the solution to the wavemaker boundary value problem is represented by the velocity potential that satisfies the continuity equation $\nabla \cdot \nabla \phi=0$ (Equation 2.1). In the new coordinate system introduced in Figure 5.1, the Bottom Boundary Equation (BBC) becomes

$$
\frac{\partial \phi}{\partial z}=0 \quad \text { on } z=0
$$

By taking the Taylor series expansion about $\phi(x, z=h, t)$ about $z=0$ and neglecting all second order terms and subsequent higher order terms for being very small, the two linearized free surface boundary conditions (DFSBC and KFSBC) are combined 
CHAPTER 5. DEVELOPMENT AND QUANTIFICATION OF A WAVE

GENERATOR AND SHIP MOTION SIMULATOR

to form the Combined Free Surface Boundary Condition (CFSBC),

$$
\frac{\partial \phi}{\partial z}-\frac{\omega^{2}}{g} \phi=0 \quad \text { on } z=h .
$$

For a wedge oscillating with motion $S \sin (\omega t)$, the normal component of the wedge velocity is $\omega S \sin (\beta) \cos (\omega t)$. To ensure that the fluid velocity normal to the wedge surface is equal to the normal velocity component of the wedge along $\overrightarrow{\mathbf{n}}$, it follows that the Kinematic Boundary Condition (KBC) on the wavemaker be defined as

$$
\begin{array}{rrr}
\frac{\partial \phi}{\partial n}=\omega S \sin (\beta) \cos (\omega t) & \text { at } d^{\prime} \leq z \leq h \\
\frac{\partial \phi}{\partial x}=0 & \text { at } 0 \leq z<d^{\prime}
\end{array}
$$

when assuming no leakage around the wedge. Equation 5.3a describes the KBC on the inclined wedge surface $d^{\prime} \leq z \leq h$ and Equation 5.3b describes the KBC on the clearance between the channel bottom and wedge bottom $0 \leq z<d^{\prime}$. However, when $\beta=0$, Equation 5.3a also satisfies Equation 5.3b and is therefore sufficient for describing the $\mathrm{KBC}$ on the wavemaker on $0<z<h$. In the $x-z$ coordinate system, Equation 5.3a becomes

$$
\frac{\partial \phi}{\partial x}-\frac{\partial \phi}{\partial z} \tan (\beta)=\omega S \tan (\beta) \cos (\omega t) \quad \text { at } x=\left(z-d^{\prime}\right) \tan (\beta)
$$

The radiation condition must also be applied to ensure that, at large distances from the wavemaker, the disturbed surface takes the form of a progressive wave [24].

To examine the radiation condition, the velocity potential $\phi(x, z, t)$ can be expressed in its separable form of

$$
\Phi(x, z, t)=\operatorname{Re}\left[\phi(x, z) e^{-i \omega t}\right]
$$

where $\phi(x, y) \cos (\omega t)=\operatorname{Re}\left[\phi(x, y) e^{-i \omega t}\right]$, and by using the method of separation of variables, one solution to the Laplace equation that satisfies both boundary conditions 


\subsection{THEORETICAL ANALYSIS OF A PLUNGER-TYPE WAVEMAKER}

expressed in equations 5.1 and 5.2 and the radiation condition is given by

$$
\Phi=A_{0} \cosh \left(k_{p} z\right) e^{i k_{p} x}+\sum_{n=1}^{\infty} A_{n} \cos \left(k_{n} z\right) e^{-k_{n} x}
$$

where, similar to linear wave theory, dispersion relationships must hold for both

$$
\begin{aligned}
& \omega^{2}=g k_{p} \tanh \left(k_{p} h\right) \\
& \omega^{2}=-g k_{n} \tan \left(k_{n} h\right), \quad n=1,2, \ldots, \infty .
\end{aligned}
$$

Equation 5.6 represents the potential function of one progressive wave that propagates in the positive $x$ direction and an infinite number of standing waves that decay exponentially with distance from the wavemaker. The wave numbers $k_{p}$ and $k_{n}$ are for progressive wave and standing waves respectively, and $A_{0}$ and $A_{n}$ are coefficients that must be solved using the KBC on the wavemaker. Substituting Equation 5.6 into Equation 5.4 gives the full $\mathrm{KBC}$,

$$
\begin{aligned}
& {\left[i A_{0} k_{p} \cosh \left(k_{p} z\right) e^{i k_{p}\left(z-d^{\prime}\right) \tan (\beta)}-\sum_{n=1}^{\infty} A_{n} k_{n} \cos \left(k_{n} z\right) e^{-k_{n}\left(z-d^{\prime}\right) \tan (\beta)}\right]-} \\
& {\left[A_{0} k_{p} \sinh \left(k_{p} z\right) e^{i k_{p}\left(z-d^{\prime}\right) \tan (\beta)}-\sum_{n=1}^{\infty} A_{n} k_{n} \sin \left(k_{n} z\right) e^{-k_{n}\left(z-d^{\prime}\right) \tan (\beta)}\right] \tan (\beta)} \\
& =\omega S \tan (\beta) .
\end{aligned}
$$

To solve for the coefficients $A_{0}$ and $A_{n}$, Wu proposed a semi-analytical solution using the boundary collocation method [24]. The boundary collocation method uses a linear combination of functions that are solutions to the governing equation. Unknown coefficients are then found by least squares matching of the remaining boundary conditions at discrete points on the boundary [46]. For the wavemaker problem, the boundary is the inclined surface of the wedge and the clearance between the wedge bottom and the bottom of the channel. The inclined surface of the wedge and clearance is divided into $M-1$ segments of equal vertical length with two node points at each end of the segments totalling $M$ nodes. According to the boundary 
CHAPTER 5. DEVELOPMENT AND QUANTIFICATION OF A WAVE

GENERATOR AND SHIP MOTION SIMULATOR

collocation method, Equation 5.8 must be satisfied at all $M$ node points, which is represented in matrix form as

$$
\mathrm{B}_{\mathrm{mn}} \cdot \mathrm{A}_{\mathrm{n}}^{\prime}=\mathrm{D}_{\mathbf{m}}
$$

where the elements of matrices $\mathbf{B}_{\mathbf{m n}}, \mathbf{A}_{\mathbf{n}}^{\prime}$ and $\mathbf{D}_{\mathbf{m}}$ are expressed by $\mathrm{Wu}[24]$ as

$$
\begin{aligned}
B_{m 1} & =k_{p} h\left\{i \cosh \left[k_{p} h\left(\frac{z}{h}\right)_{m}\right]-\tan (\beta) \sinh \left[k_{p} h\left(\frac{z}{h}\right)_{m}\right]\right\} e^{\left[i k_{p} h\left(\frac{z-d^{\prime}}{h}\right)_{m} \tan (\beta)\right]} \\
B_{m n} & =-k_{n-1} h\left\{\cos \left[k_{n-1} h\left(\frac{z}{h}\right)_{m}\right]-\tan (\beta) \sin \left[k_{n-1} h\left(\frac{z}{h}\right)_{m}\right]\right\} \cdot \\
& e^{\left[-k_{n-1} h\left(\frac{z-d^{\prime}}{h}\right)_{m} \tan (\beta)\right]}, \quad n \neq 1 \\
A_{1}^{\prime} & =\frac{A_{0}}{\omega S h} \\
A_{n}^{\prime} & =\frac{A_{n-1}}{\omega S h}, \quad n \neq 1 \\
D_{m} & =\tan (\beta)
\end{aligned}
$$

Following the boundary collocation method, Equation 5.9 is solved in the least squares sense to minimize the sum of the quadratic error by multiplying by the Hermitian transpose of $\mathbf{B}$, denoted $\mathbf{B}^{T}$, on both sides of Equation 5.9, such that

$$
\mathbf{B}^{T} \cdot \mathbf{B} \cdot \mathbf{A}^{\prime}=\mathbf{B}^{T} \cdot \mathbf{D}
$$

Wu suggests using $M=200$ collocation points and a finite number of $N=16$ for obtaining an approximate solution to Equation 5.11 such that one progressive wave and 15 standing waves are included in the solution [24]. The standing waves decay with distance from the wave channel, and for a semi-infinite wave channel, it is assumed that only the progressive waves will exist at a large distace $x$. At this large distance $x$, the potential function in Equation 5.5 is expressed as

$$
\phi=\operatorname{Re}\left[A_{0} \cosh \left(k_{p} z\right) e^{i\left(k_{p} x-\omega t\right)}\right]
$$


and, similar to Equation 2.8, the position of the free surface is

$$
\eta(x, t)=\frac{1}{g} \frac{\partial \phi}{\partial t}=\operatorname{Re}\left[\frac{-i \omega A_{0}}{g} \cosh \left(k_{p} h\right) e^{i\left(k_{p} x-\omega t\right)}\right] .
$$

Finally, the wave amplitude $a$ to stroke ratio $S$ is derived to be

$$
\frac{a}{S}=\left|-i A_{1}^{\prime} k_{p} h \sinh \left(k_{p} h\right)\right| .
$$

The wave amplitude to stroke ratio provides a metric that is useful in wavemaker design. For a desired wave amplitude $a=H / 2$, one can infer the required wavemaker stroke amplitude from the amplitude to stroke ratio expressed in Equation 5.14. Figure 5.2 plots the amplitude to stroke ratio against the dimensionless frequency parameter $k_{p} b$ for various wedge aspect ratios $d / b$. From Figure 5.2, wedges

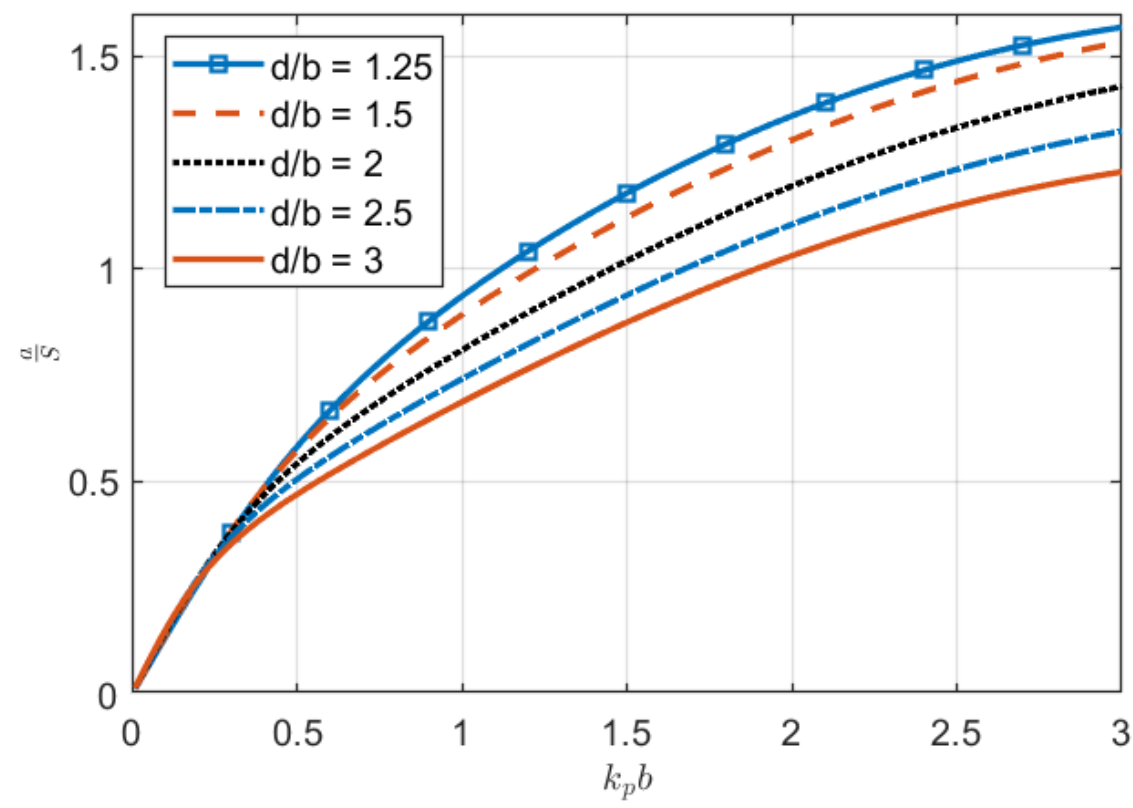

Figure 5.2: Amplitude to stroke ratio vs dimensionless frequency parameter $k_{p} b$ for various wedge shapes where $d=0.5 \mathrm{~m}$ and $\mathrm{h}=1.0 \mathrm{~m}$.

with a smaller aspect ratio $d / b$ have a larger wave amplitude to stroke ratio $a / S$, that is, in the physical sense of wavemaker design, will require a smaller actuated stroke length to achieve a desired wave amplitude. 
CHAPTER 5. DEVELOPMENT AND QUANTIFICATION OF A WAVE

GENERATOR AND SHIP MOTION SIMULATOR

As the standing waves decay exponentially with distance away from the wavemaker, it follows that only the progressive wave will exist at the far field of the water channel [24]. While this approximation is reasonable to assume for the semiinfinite wave channel in Wu's formulation, the assumption must be verified for the finite length of the flume tank test section. Observing the standing wave portion in Equation 5.6, $A_{n} \cos \left(k_{n} z\right) e^{-k_{n} x}$, the first term at $n=1$ is expected to decay the slowest, at a rate of $e^{-k_{1} x}$. Figure 5.3 illustrates the dispersion relationship for standing wave modes defined in Equation $5.7 \mathrm{~b}$, where the dimensionless value $k_{n} h$ is isolated on both sides of Equation 5.7b. The abscissa of the intersection points between the plot of $-\tan \left(k_{n} h\right)$, solid lines, and the curve $\frac{\omega^{2} h}{g k_{n} h}$, dashed line, provide a means of obtaining the wave number of each mode for a given water depth $h$. Referring to Figure 5.3 , the value of $k_{1} h$ must be greater than the - tan $\left(k_{n} h\right)$ asymptote at $\frac{\pi}{2}$; however, $k_{1}$ will be conservatively set to $\frac{\pi}{2}$ for the following analysis and thus the decay of standing wave height for the first standing wave mode will be greater than $e^{-\left(\frac{\pi}{2}\right)\left(\frac{x}{h}\right)}$.

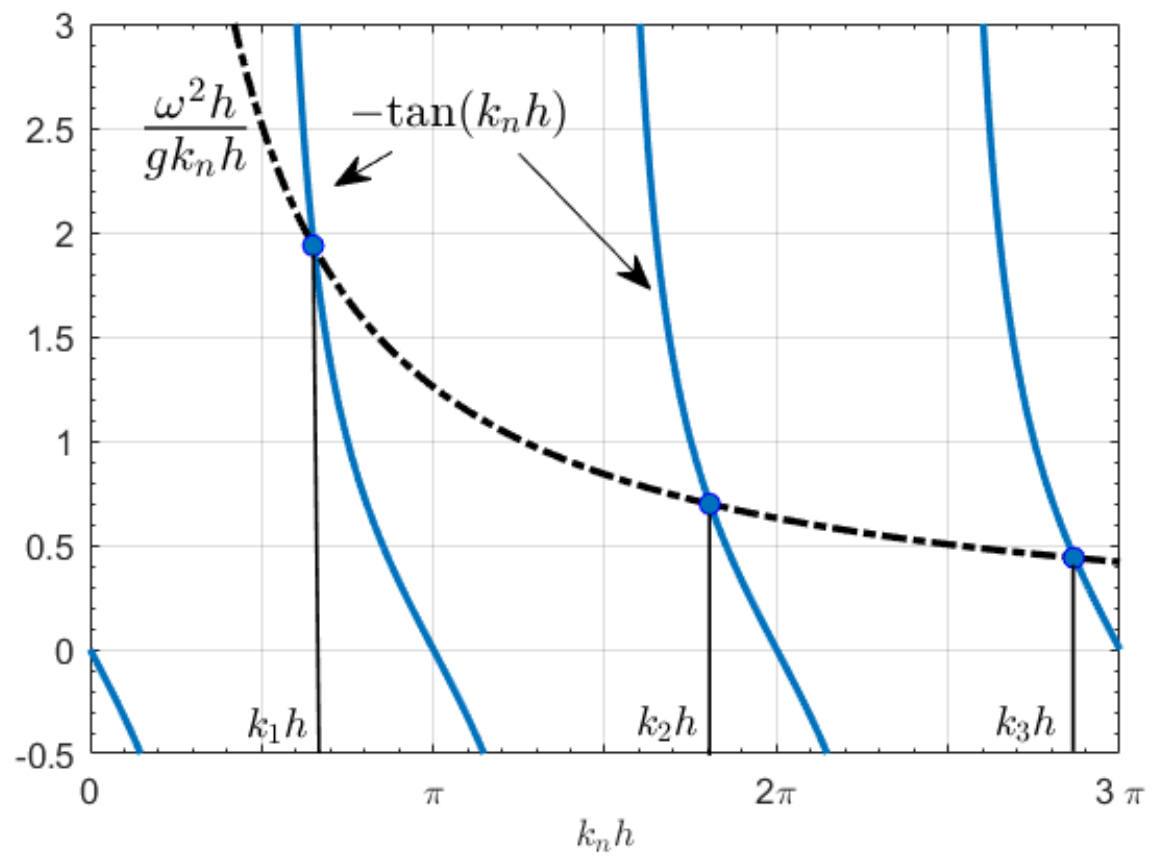

Figure 5.3: Graphical representation of the dispersion relationship for the standing wave modes. Here, $\omega=7.4 \mathrm{rad} / \mathrm{s}$ and $\mathrm{h}=71 \mathrm{~cm}$. 


\subsection{THEORETICAL ANALYSIS OF A PLUNGER-TYPE WAVEMAKER}

At a distance of $x=2 h$ from the wavemaker, $e^{-\left(\frac{\pi}{2}\right)\left(\frac{x}{h}\right)}=0.043$ and at a distance of $x=3 h$ from the wavemaker, $e^{-\left(\frac{\pi}{2}\right)\left(\frac{x}{h}\right)}=0.0089$. Therefore, at a distance away from the wavemaker that is double or triple the water depth of the water channel, the first term in the standing wave series can be considered negligible compared to the progressive wave term. In the flume tank, this translates to a distance of $1.4 \mathrm{~m}$ to $2.1 \mathrm{~m}$ away from the wavemaker. With a test section length of $1.8 \mathrm{~m}$ in the flume tank, sufficient decay of the standing waves should be possible.

\subsubsection{Investigating the effects of current on wavemaker de- sign}

Relative flow velocity of the submerged body relative to the sea is an important parameter in the investigation of towed body and recovery operations. In the simulations in Chapter 3, this velocity was set to the mean ship velocity. In flume tank experiments, the relative flow velocity will be emulated by circulating the water in the flume tank at a uniform flow speed. It is therefore important to investigate the effects that the flow speed will have on the generation of waves in the tank.

The impact of sea currents on wave heights is an observed phenomenon that has been investigated in many sea conditions. Viitak et al. [47] studied the effects of surface currents and sea level on significant wave heights in the Eastern Baltic Sea and found a clear effect of surface currents on wave field evolution where increases and decreases of $20 \%$ significant wave heights were seen in both the model and in situ wave measurements. An increase in significant wave height was observed when the waves and current were propagating in opposite directions, while a decrease in significant wave height was observed for waves and current propagating in the same direction. Ardhuin et al. [48] also demonstrated that variations in current are the main source of variations in wave height at the same scale.

The boundary value problem was briefly investigated for regular waves in 
CHAPTER 5. DEVELOPMENT AND QUANTIFICATION OF A WAVE

GENERATOR AND SHIP MOTION SIMULATOR

section 2.3.1 where the flow potential for a propagating wave with a uniform flow current $U_{o}$ is given in Equation 2.12. A similar solution could be found for the wavemaker problem; however, when extending this boundary value problem to wavemaker theory, it is more convenient to adopt a reference frame that moves with the flow current $U_{o}$. In this moving reference frame, there is no flow current and the solution to the wavemaker boundary value problem for the no current condition found in the previous section can be used [9]. Relating the moving reference frame to the stationary one requires that the wavelength be the same in both systems and that the period $T$ relative to the stationary frame relates to the period $T^{\prime}$ of the moving reference frame by

$$
T=T^{\prime}\left(1-\frac{U_{o}}{C}\right)
$$

where the celerity $C$ is obtained from the dispersion relationship and replacing $\omega$ with $k C$

$$
\left(C-U_{o}\right)^{2}=\frac{g}{k} \tanh (k h) .
$$

Dean and Dalrymple [9] solved Equation 5.16 using the quadratic solution and replacing the wave number $k$ with $\omega / C$, from the definition of phase velocity where $C=\omega / k$, such that

$$
C=\left(U_{o}+\frac{g}{2 \omega}\right)+\sqrt{\frac{U_{o} g}{\omega}+\frac{1}{4}\left(\frac{g}{\omega}\right)^{2}} .
$$

Applying Wu's [24] semi-analytical solution using the new moving coordinate frame, the effects of an applied uniform current on the wave amplitude to height ratio can be obtained. Figure 5.4 plots the relationship between the wave amplitude to stroke ratio $a / S$ and a uniform current $U_{o}$ flowing in the direction of the propagating wave. Figure 5.4 shows a decrease in the wavemaker amplitude to stroke ratio with increasing uniform current in the direction of propagating waves. The latter demonstrates that for a constant wavemaker stroke length, the wave amplitude propagating from the wavemaker will be smaller if a current is applied in the direction of the wave, and that as this current is increased, the wave amplitude of the gener- 


\subsection{THEORETICAL ANALYSIS OF A PLUNGER-TYPE WAVEMAKER}

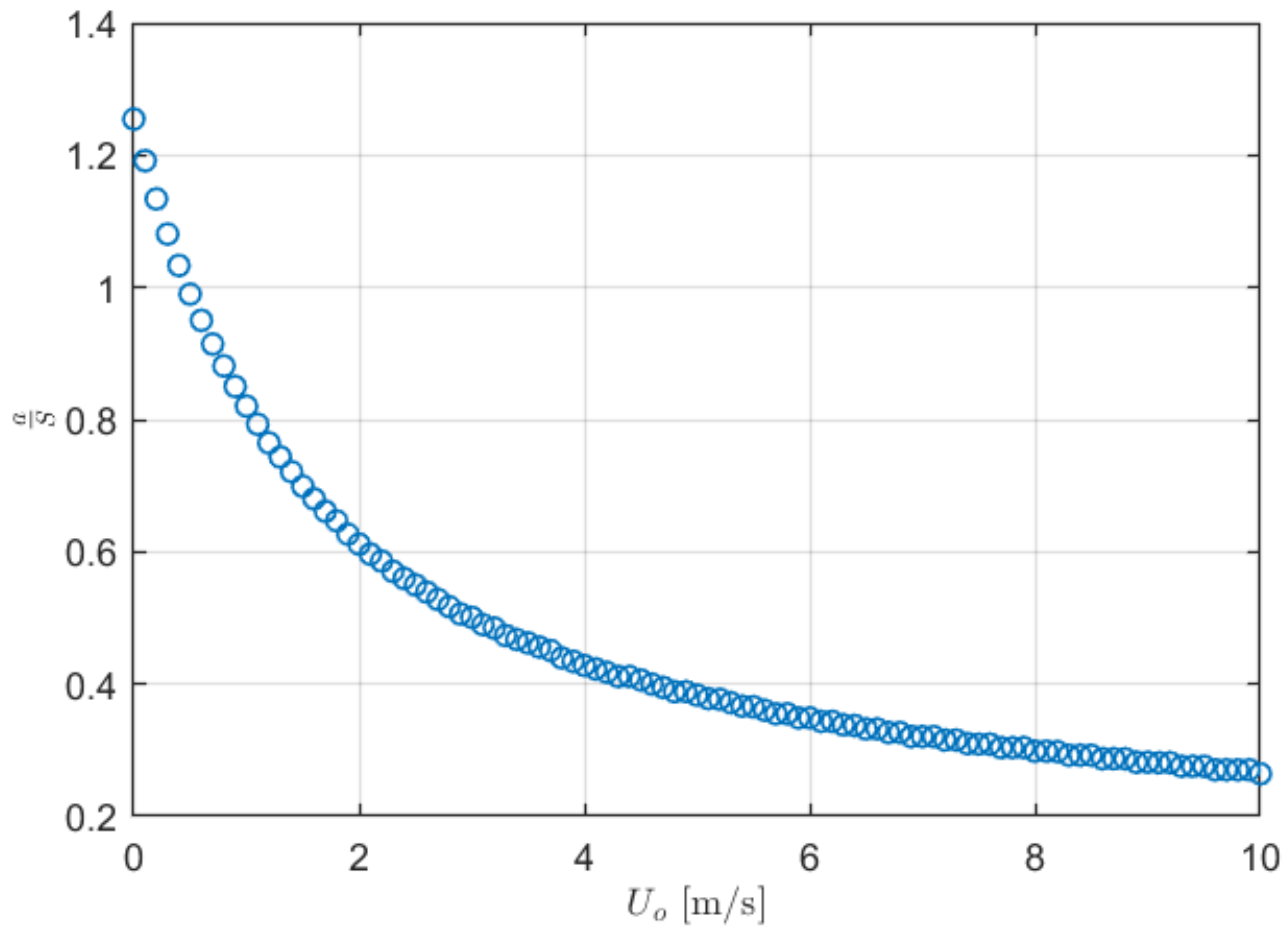

Figure 5.4: Relationship between a uniform current and the wave amplitude to stroke ratio of a plunger-type wavemaker. Here, $\omega=7.4 \mathrm{rad} / \mathrm{s}$ and $\mathrm{h}=1 \mathrm{~m}$.

ated wave will decrease for the fixed stroke length $S$. This observation from Figure 5.4 is similar to observations made by Viitak et al. [47], where a decrease in significant wave height was observed when currents flowing in the direction of propagating waves was present. For purposes of plunger wavemaker design, the boundary collocation method is judged to be acceptable for describing the plunger wavemaker-wave interactions with uniform current based on the analysis performed above; however, experimental validation will be required to verify the accuracy of the model.

In terms of wavemaker design, Figure 5.2 is useful as it provides a means to analyze different plunger wedge geometries once a wavemaker operating point is selected. In the next section, the selection of the desired wavemaker operating point for the flume tank will be discussed. Figure 5.2 will also be advanced to include the effects of expected operating currents in the flume tank in order to develop a design for a wavemaker. 
CHAPTER 5. DEVELOPMENT AND QUANTIFICATION OF A WAVE

GENERATOR AND SHIP MOTION SIMULATOR

\subsection{Conceptual design of a plunger-type wavemaker}

To address the third objective of this thesis, a design method for designing a plungertype wavemaker is presented in this section. Selecting the operating point for the plunger wavemaker design requires knowledge of the water channel's operational conditions at the location where the wavemaker will be implemented, as well as the desired amplitudes and periods of the waves to be generated in the channel. The water depth of the flume tank test section at Carleton University can be adjusted up to $h=86 \mathrm{~cm}$ and is typically filled to $h=71 \mathrm{~cm}$. The maximum current at which the water can circulate in the flume tank is specified as $0.305 \mathrm{~m} / \mathrm{s}$.

The wavemaker is to reproduce flume scale sea states 3 to 6 that are described by ranges of significant wave heights and modal periods in Table 2.2. To select an operating point representative of the ranges of conditions encountered in sea states 3 to 6 , the modal period $T_{p}=0.85 \mathrm{~s}$ is selected as it is both near the average modal period of $0.80 \mathrm{~s}$ across the four sea states, and it occurs in the modal period ranges of each sea state of interest. A modal period of $0.85 \mathrm{~s}$ gives a wave frequency of $\omega=7.392 \mathrm{rad} / \mathrm{s}$. With known values for $h, \omega, g$ and $U_{o}$, the progressive wave number $k_{p}$ can be computed using Equations 5.16 and 5.17.

Figure 5.5 advances Figure 5.2 to include the effects of flow current on the wavemaker design. In Figure 5.5, the amplitude to stroke ratio $a / S$ is plotted vs the dimensionless frequency parameter $k_{p} b$ for flow currents $U_{o}$ that are represented by various line styles and for different plunger wedge aspect ratios $d / b$.

Two parameters must be selected in order to set the design set point. The parameters may be later changed during design iteration, but must be initially set to provide a start point for the design process. The parameters to be selected are the the amplitude to stroke ratio $a / S$ and the plunger wedge aspect ratio $d / b$. These parameters drive the actuator sizing requirements for the wavemaker. Important considerations when sizing the actuator for the wavemaker are the resolution of the 


\subsection{CONCEPTUAL DESIGN OF A PLUNGER-TYPE WAVEMAKER}

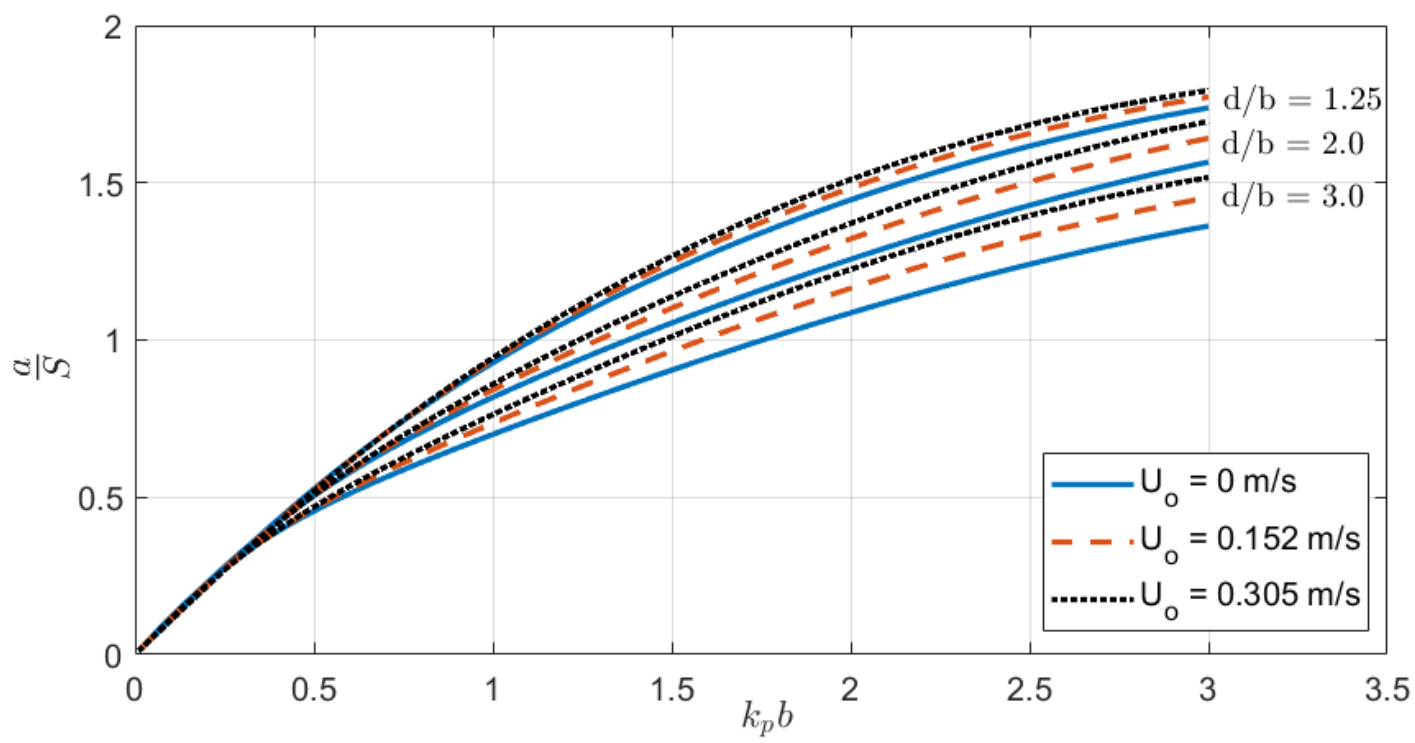

Figure 5.5: Amplitude to stroke ratio vs dimensionless frequency parameter $k_{p} b$ for various wedge shapes and current $U_{o}$ where $\mathrm{h}=71 \mathrm{~cm}$ and $T=0.85 \mathrm{~s}$.

plunger amplitude to stroke ratio and the travel speed of the plunger actuator $V_{a}$. The following procedure summarizes the plunger-type wavemaker design process using Figure 5.5 as a design tool to size the actuator and define the plunger wedge shape.

a. Set the operating parameters that describe the test environment: flow current $U_{o}$, water depth $h$, wave period $T_{p}$ and the maximum desired wave amplitude a, e.g. $a=H_{s} / 2$.

b. Solve for the wave number $k_{p}$ using Equation 5.17 .

c. Select a desired amplitude to stroke ratio $a / S$. An amplitude to stroke ratio that is less than 1 yields a greater resolution for actuator displacement, but can also limit the maximum wave amplitude that can be created by the actuator.

d. Select a plunger wedge aspect ratio $d / b$ to start. From Figure 5.5 , larger $d / b$ ratios correspond to smaller $a / S$ ratios, which as mentioned in the step above is desirable for greater resolution in actuator displacement.

e. Find $k_{p} b$ on the abscissa of Figure 5.5 at the point where the amplitude to stroke 
CHAPTER 5. DEVELOPMENT AND QUANTIFICATION OF A WAVE

GENERATOR AND SHIP MOTION SIMULATOR

ratio $a / S$ intersects the $d / b$ curve. The operating point is now defined as the point $\left(k_{p} b, a / S\right)$.

f. Solve for the plunger width $b=k_{p} b / k_{p}$ and plunger length $d=d / b * b$. The wedge length $d$ must not exceed the actuator stroke length $S$.

g. The required actuator travel speed $V_{a}$ is given by $V_{a}=\frac{2 a}{T_{m}} \frac{1}{(a / S)}$.

h. Select an actuator that meets the requirements $S \geq d$ and $V_{a} \geq \frac{2 a}{T_{m}} \frac{1}{(a / S)}$.

i. Evaluate the actuator stroke length $S$ and travel speed $V_{a}$ against all desired test conditions to be produced by the wavemaker by reiterating through steps a. to h.

Figure 5.6 shows a conceptual design of the plunger-type wavemaker. It consists of a vertical structure built with extruded t-slotted aluminum members attached to a moving carriage that glides vertically along the rails on four sleeve bearings. The plunger wedge is rigidly attached to the carriage and is the only component that is submerged. In future work, the wavemaker design will be finalized according to the procedure outlined above, and will be tested in the flume tank. The next section will present the design of the robotic ship motion simulator.

\subsection{Design overview of a robotic ship motion sim- ulator}

The second objective of this thesis was to develop and quantify a ship motion simulator for the flume tank laboratory at Carleton University. As briefly discussed in Chapter 2, this ship motion simulator must produce motion in 5 degrees-of-freedom: the translations surge, sway and heave, and the roll and pitch rotations depicted in Figure 2.2. Requirements for the total displacement of each degree-of-freedom are summarized in Table 5.1 as well as the desired payload mass to be moved by the ship 


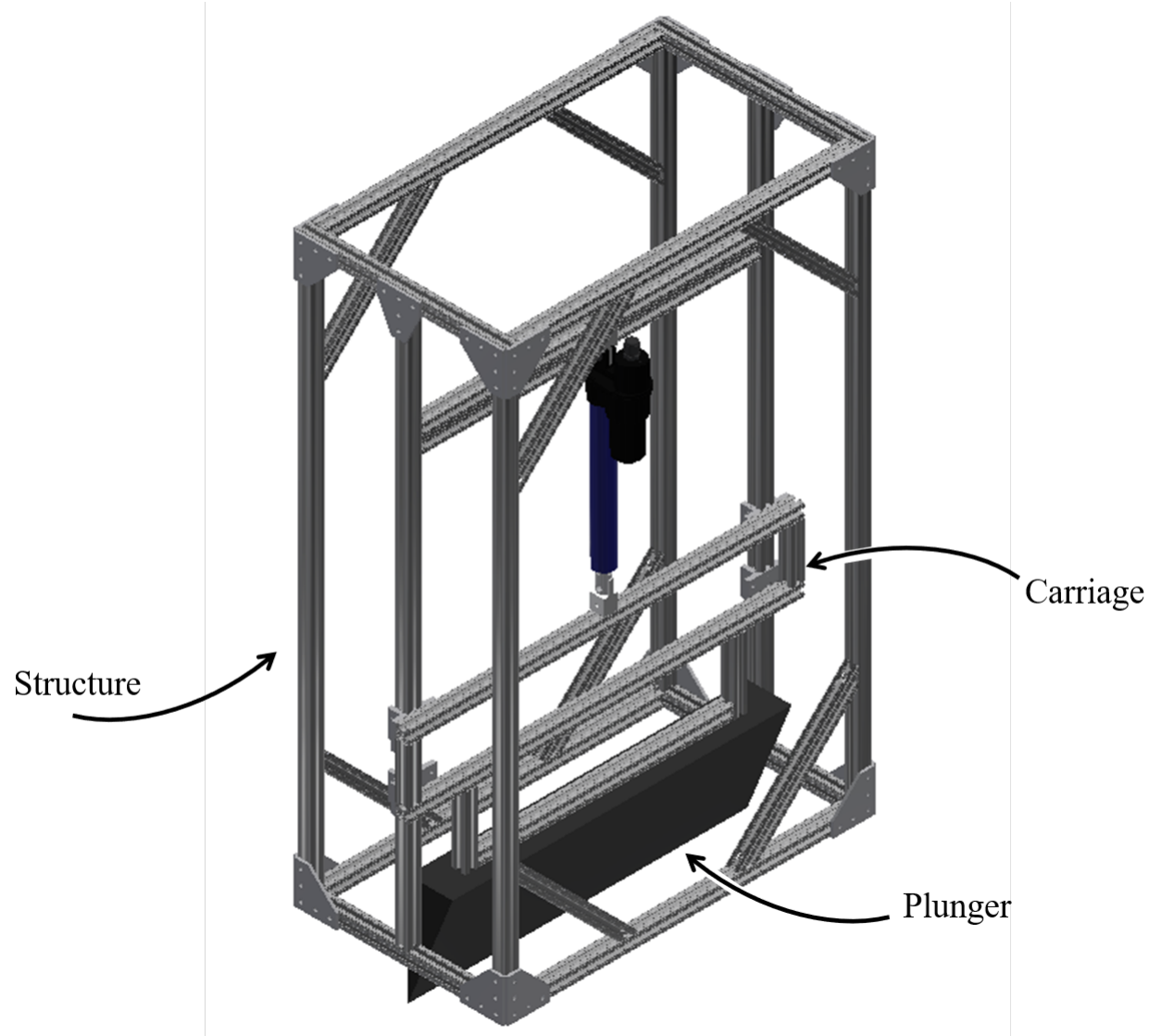

Figure 5.6: Conceptual design of a plunger-type wavemaker for the Carleton University flume tank.

motion simulator. The desired displacement values were selected such that scaled ship motion data could be reasonably emulated based on the size constraints of the flume tank test section. In addition to the requirements in Table 5.1, the ship motion simulator must also be corrosion resistant as it will operated in an environment where water spray can occur. A desired IP rating of IP67 or higher was therefore specified.

\begin{tabular}{|l|l|}
\hline Design Requirement & Specification \\
\hline Surge Displacement & $200[\mathrm{~mm}]$ \\
\hline Sway Displacement & $200[\mathrm{~mm}]$ \\
\hline Heave Displacement & $200[\mathrm{~mm}]$ \\
\hline Roll Displacement & $\pm 20[\mathrm{deg}]$ \\
\hline Pitch Displacement & $\pm 20[\mathrm{deg}]$ \\
\hline Payload Mass & $25[\mathrm{~kg}]$ \\
\hline
\end{tabular}

Table 5.1: Design requirements for a 5 DOF robotic ship motion simulator. 
CHAPTER 5. DEVELOPMENT AND QUANTIFICATION OF A WAVE

GENERATOR AND SHIP MOTION SIMULATOR

The final design of the robotic ship motion simulator is shown in Figure 5.7 and consists of an aluminum structure that supports a parallel manipulator on a translating gantry system base. On the left of Figure 5.7 is the final CAD model, and on the right is the final build assembly of the ship motion simulator mounted to the flume tank test section via C-clamps. The technical drawing package for the ship motion simulator is included in Appendix B. The surge and sway displacements are achieved with a gantry system that moves in the $\mathrm{x}-\mathrm{y}$ horizontal plane. The heave, roll and pitch motion are achieved via a 3-DOF parallel manipulator that hangs below the gantry system. The bottom structure is built up to provide enough clearance above the mean water level.

Top view representations of the surge-sway gantry system are shown in Figure 5.8. Figure 5.8a depicts the gantry system in its initial surge and sway positions and Figure 5.8b depicts the gantry system at the maximum surge and sway displacements. The surge and sway motion are independently actuated by linear actuators that provide a maximum displacement of $200 \mathrm{~mm}$ in both the surge and sway directions. The carriage sits on four sleeve bearings and moves along aluminum rails with bearing pads that resist dirt and water. The carriage supports the base of the 3 -DOF heave motion platform which hangs below the carriage, as illustrated in Figure 5.7. In the following section, a kinematic analysis of the heave motion platform is provided.

\subsection{Kinematic analysis and control scheme}

The heave motion platform provides motion in heave, roll and pitch. Figure 5.9 shows the 3-DOF motion platform design consisting of a top base platform attached to the carriage of the surge-sway gantry system to which three linear actuators are attached via pinned joints, and a bottom end-effector platform which is attached to the three actuators via universal joints. One important requirement for the motion platform is that the roll and pitch motion of the end effector be about the $\mathrm{x}$ and $\mathrm{y}$ axes of 

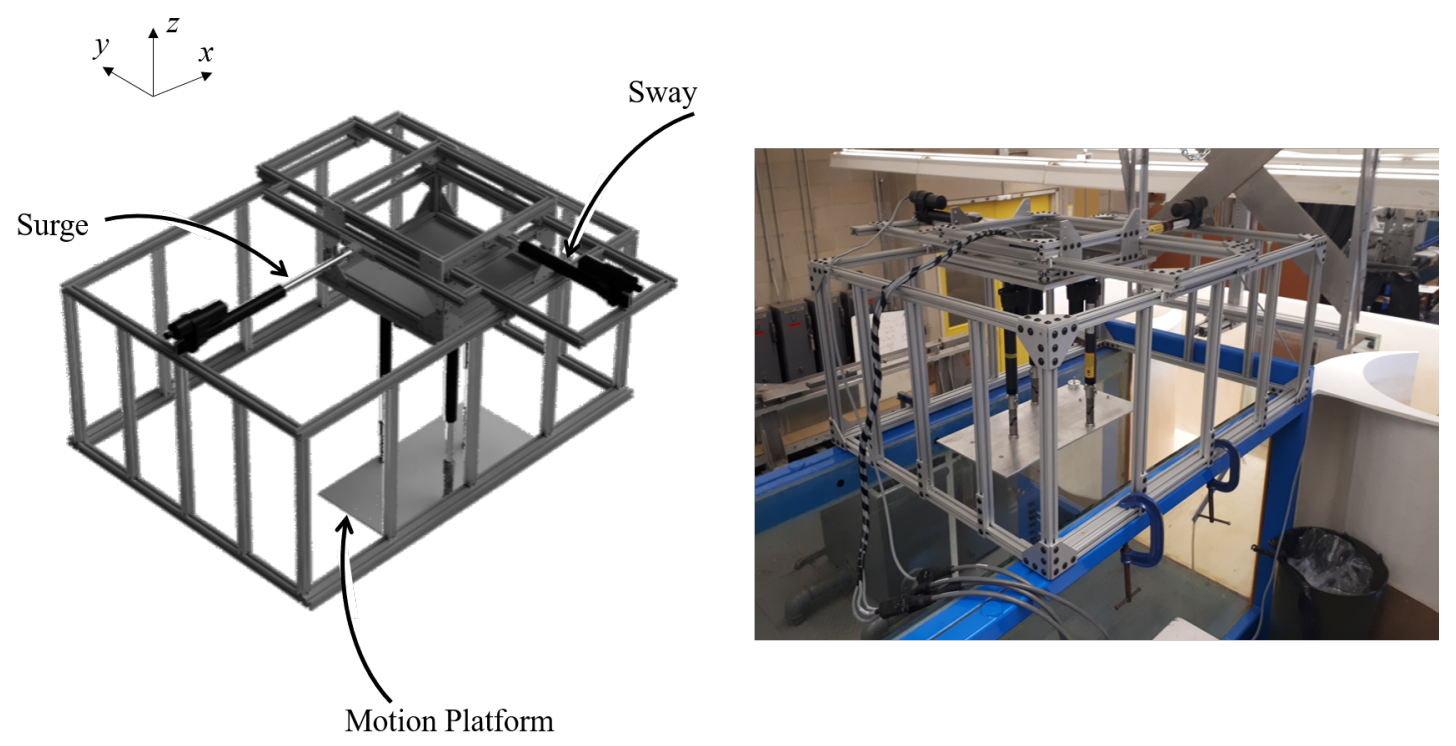

Figure 5.7: CAD model representation (left) and physical assembly (right) of the robotic ship motion simulator designed and built for the Carleton University flume tank.

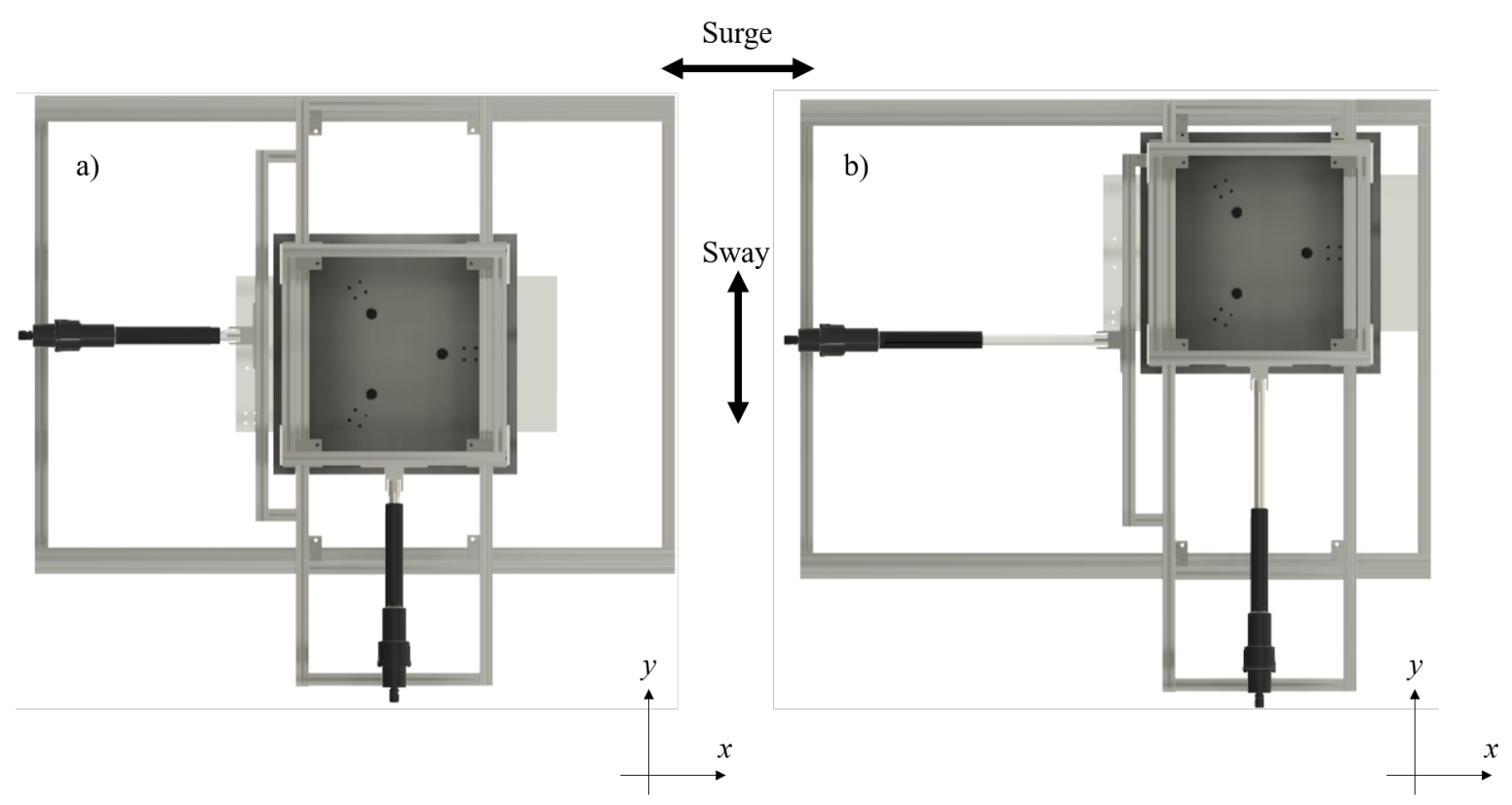

Figure 5.8: Top view representation of the surge-sway gantry system with a) showing initial positions of both axes and b) showing the end position of $200 \mathrm{~mm}$ on both axes.

the gantry system, i.e. the translation from the base frame of the platform to the end effector platform frame must at all times be only along the $\mathrm{z}$ axis. The latter requirement ensures that only pure rotation about the $\mathrm{x}$ and $\mathrm{y}$ axes occur at the 
CHAPTER 5. DEVELOPMENT AND QUANTIFICATION OF A WAVE GENERATOR AND SHIP MOTION SIMULATOR

end effector platform, in addition to heave, which must occur in order to properly emulate all five degrees-of-freedom of the ship motion about a common origin. While the surge and sway motions can be individually actuated, the inverse kinematics of the parallel manipulator are required to obtain the actuator stroke lengths necessary to achieve each heave-roll-pitch configuration.

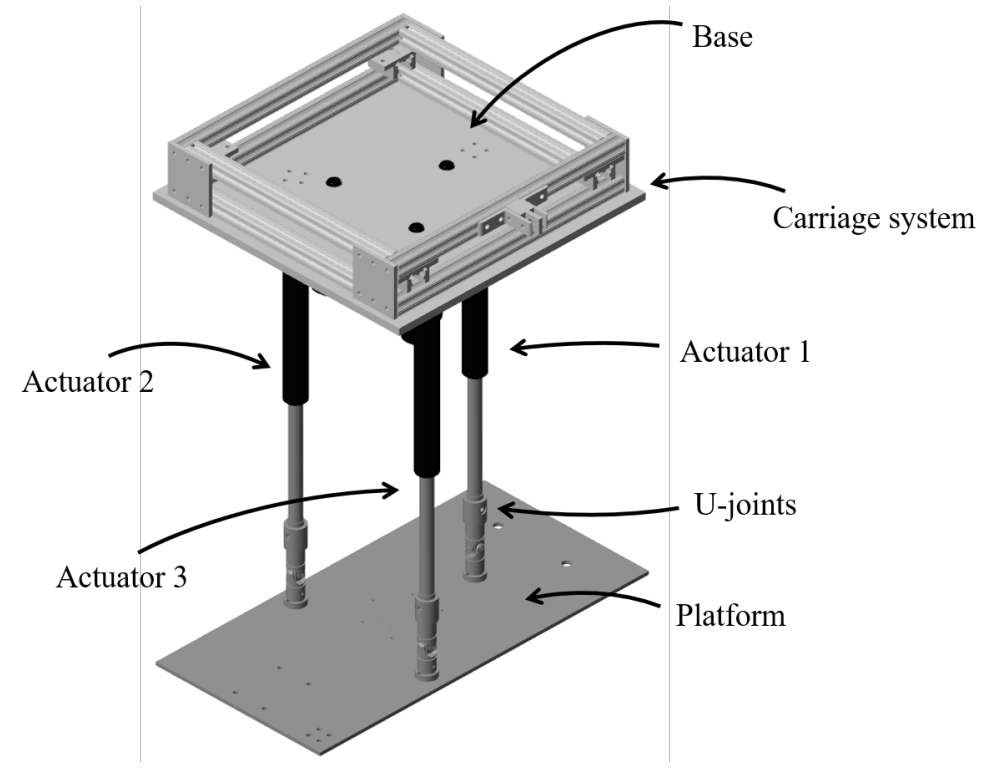

Figure 5.9: Isometric view representation of the 3-DOF motion platform. Note: support structure and the surge-sway gantry system have been removed for clarity.

\subsubsection{Inverse kinematics of a parallel manipulator}

For the purpose of analyzing the kinematic model of the parallel manipulator, two relative coordinate frames are assigned. Figure 5.10 shows the schematic of the kinematic model with the base Cartesian coordinate frame $X_{B} Y_{B} Z_{B}$ assigned to the centre of the base at point $O_{B}$, which is equivalent to the surge-sway gantry system coordinate frame, and a platform coordinate frame $X_{P} Y_{P} Z_{P}$ at the centre of the end-effector platform at point $O_{P}$. The pinned joints are located at points $B_{1}, B_{2}$ and $B_{3}$ and the driven universal joints at the platform are located at points $P_{1}, P_{2}$ and $P_{3}$. The length of the $i^{\text {th }} \operatorname{leg}, l_{i},(i=1,2,3)$ represents the displacement of the driving prismatic 


\subsection{KINEMATIC ANALYSIS AND CONTROL SCHEME}

joints. $l_{o}$ represents the initial retracted length of the actuators and $r_{b}$ and $r_{p}$ are the radii of the base and platform passing through the joints at $B_{i}$ and $P_{i}$ respectively.

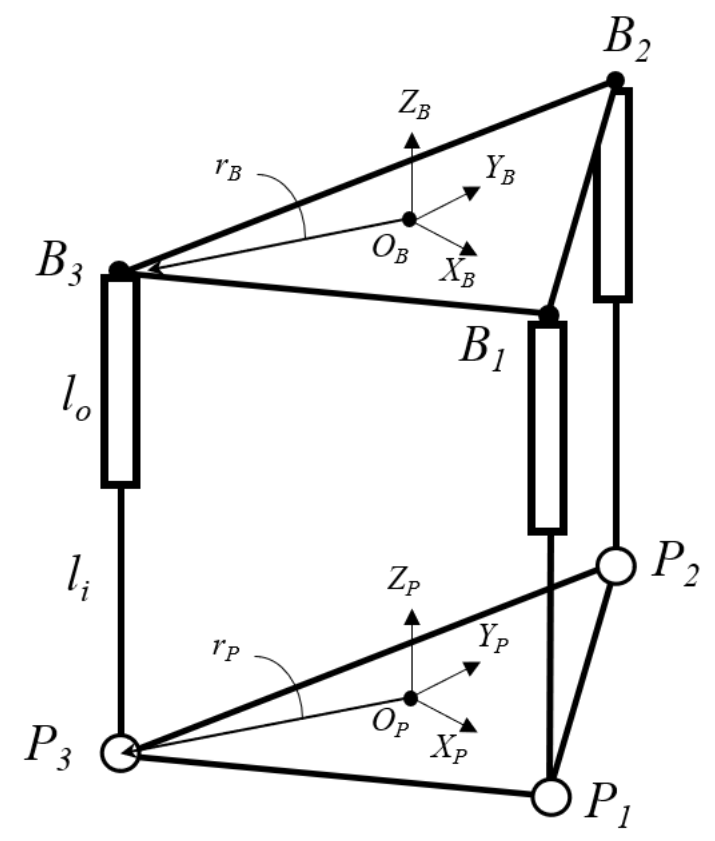

Figure 5.10: Kinematic model for a 3-DOF parallel kinematic manipulator.

The inverse kinematics of the 3-DOF heave motion platform will be used to determine the actuator displacements $l_{i}$ required to achieve the desired motion platform configuration. The kinematic analysis of a Stewart platform given by Liu et al. [49] has been modified to calculate only three leg lengths, with joint geometry influenced by $\mathrm{Ng}$ et al. [50].

Similar to $\mathrm{Ng}$ et al. [50], the positions of $B_{i}$ in the base frame $X_{B} Y_{B} Z_{B}$ are equally spaced at $120^{\circ}$ of each other are expressed by vectors $\overrightarrow{b_{i}}$

$$
\overrightarrow{b_{1}}=\left[r_{b}, 0,0\right]^{T}, \quad \overrightarrow{b_{2}}=\left[-\frac{1}{2} r_{b}, \sqrt{\left(\frac{3}{2}\right)}, 0\right]^{T}, \quad \overrightarrow{b_{3}}=\left[-\frac{1}{2} r_{b},-\sqrt{\left(\frac{3}{2}\right)}, 0\right]^{T}
$$

and, likewise, the positions of $P_{i}$ in the moving platform frame $X_{P} Y_{P} Z_{P}$ are expressed 
CHAPTER 5. DEVELOPMENT AND QUANTIFICATION OF A WAVE GENERATOR AND SHIP MOTION SIMULATOR

by vectors $\overrightarrow{p_{i}}$

$$
\overrightarrow{p_{1}}=\left[r_{p}, 0,0\right]^{T}, \quad \overrightarrow{p_{2}}=\left[-\frac{1}{2} r_{p}, \sqrt{\left(\frac{3}{2}\right)}, 0\right]^{T}, \quad \overrightarrow{p_{3}}=\left[-\frac{1}{2} r_{p},-\sqrt{\left(\frac{3}{2}\right)}, 0\right]^{T}
$$

where, for the designed heave motion platform, $r_{B}=r_{P}=140.5 \mathrm{~mm}$.

The homogeneous transformation ${ }_{B}^{P} \mathbf{T}$ describes the transformation from the platform frame, superscript $\mathrm{P}$, to the base frame, subscript $\mathrm{B}$, and has the form

$$
{ }_{B}^{P} \mathbf{T}=\left[\begin{array}{cccc} 
& { }_{B}^{P} \mathbf{R}_{3 \times 3} & & q_{x} \\
& & & q_{y} \\
& & & q_{z} \\
0 & 0 & 0 & 1
\end{array}\right]
$$

where the translation vector from the platform to the base is defined by ${ }^{B} \vec{q}=$ $\left[q_{x}, q_{y}, q_{z}\right]^{T}$ and ${ }_{B}^{P} \mathbf{R}$ is the $3 \times 3$ rotation matrix from the platform frame to the base frame following the pitch-roll-yaw $(\theta, \phi, \psi)$ convention given by [51]

$$
{ }_{B}^{P} \mathbf{R}=\left[\begin{array}{ccc}
\cos \theta \cos \psi & \cos \theta \sin \psi & -\sin \theta \\
\sin \phi \sin \theta \cos \psi-\cos \phi \sin \psi & \sin \phi \sin \theta \sin \psi+\cos \phi \cos \psi & \cos \theta \sin \phi \\
\cos \phi \sin \theta \cos \psi+\sin \phi \sin \psi & \cos \phi \sin \theta \sin \psi-\sin \phi \cos \psi & \cos \theta \cos \phi
\end{array}\right]
$$

Yaw $\psi$ will always be zero as the geometry of the designed motion platform does not permit any orientation about the $\mathrm{z}$ axis. The initial $q_{z}$ value is equal to the initial fully retracted position of the actuators, such that $q_{z}(t=0)=l_{o}$.

In order to determine the leg lengths $l_{i}$, the joint positions at the base and platform must both be expressed in terms of the same reference frame [49], which in the current work is the base frame. From the transformation matrix ${ }_{B}^{P} \mathbf{T}$ defined in Equation 5.20, the coordinates of the $i^{\text {th }}$ platform joint in terms of the base frame are 


\subsection{KINEMATIC ANALYSIS AND CONTROL SCHEME}

therefore determined by the vector $\vec{P}_{i}$

$$
\left[\begin{array}{c}
\vec{P}_{i} \\
1
\end{array}\right]_{4 \times 1}={ }_{B}^{P} \mathbf{T}\left[\begin{array}{c}
\overrightarrow{p_{i}} \\
1
\end{array}\right]_{4 \mathrm{x} 1} .
$$

The leg lengths, $l_{i}$ are defined by the magnitude of the vectors between the base and platform joints. As the positions of the base and platform joints have been defined in the same reference frame, the base frame, the leg vectors are found by simply subtracting vectors $\overrightarrow{b_{i}}$ from vectors $\vec{P}_{i}$. The magnitude of the resulting leg vectors are therefore

$$
\begin{aligned}
& l_{1}=\sqrt{\left(P_{1 x}-b_{1 x}\right)^{2}+\left(P_{1 y}-b_{1 y}\right)^{2}+\left(P_{1 z}-b_{1 z}\right)^{2}}, \\
& l_{2}=\sqrt{\left(P_{2 x}-b_{2 x}\right)^{2}+\left(P_{2 y}-b_{2 y}\right)^{2}+\left(P_{2 z}-b_{2 z}\right)^{2}}, \\
& l_{3}=\sqrt{\left(P_{3 x}-b_{3 x}\right)^{2}+\left(P_{3 y}-b_{3 y}\right)^{2}+\left(P_{3 z}-b_{3 z}\right)^{2}} .
\end{aligned}
$$

The method of determining the required actuator length inputs, expressed by Equations 5.23 to 5.25, for any desired platform orientation has now been defined when given values for heave, roll and pitch. Next, the workspace of the motion platform will be analyzed to determine all possible orientations of the platform.

\subsubsection{Workspace analysis}

For this analysis, the workspace of a robot is defined as the set of positions and orientations that the robot is able to reach given the physical and mechanical limitations of the design [51]. The workspace of the 3-DOF heave platform depends on two geometric parameters: the stroke length of the prismatic link $l_{i}$ and the limitations of the universal joint angles of the platform. As the prismatic links are attached perpendicular to the base, there is no need to check for leg interference. 
CHAPTER 5. DEVELOPMENT AND QUANTIFICATION OF A WAVE

GENERATOR AND SHIP MOTION SIMULATOR

As with the actuators of the surge-sway gantry system, the stroke length of the motion platform actuators is $200 \mathrm{~mm}$. The maximum operating angle of the selected universal joints is $25^{\circ}$. Since the requirements for the ship motion simulator, summarized in Table 5.1, specify desired roll and pitch angles of $\pm 20^{\circ}$, the limiting criterion of the workspace therefore becomes the length of the prismatic links. This limiting criterion is expressed as $l_{\min }<l_{i}<l_{\max }$ where $l_{\min }$ is the minimum length of the actuators, and $l_{\max }$ is the maximum extended length of the actuators. For the actuators used for the robotic ship motion simulator, $l_{\min }=l_{o}=418.3 \mathrm{~mm}$, which includes the actuator housing and mounting brackets, and $l_{\max }=200 \mathrm{~mm}+l_{o}=$ $618.3 \mathrm{~mm}$.

An algorithm was created to compute the workspace of the motion platform. The structure of the workspace algorithm was influenced by the workspace optimization analysis performed by Badescu, Morman and Mavroidis [52], and simplifications made to this method made by Ngu et al [50] and Brecht [51]. The algorithm iterates through every possible platform orientation defined by roll and pitch angles $\phi$ and $\theta$ and heave translation $q_{z}$ at fixed steps. At each step, the platform joint positions are computed and compared with the geometric constraints outlined above. The algorithm is summarized as follows:

a. The algorithm first initializes the vectors $\overrightarrow{b_{i}}, \overrightarrow{p_{i}}$, defined in Equations 5.18 and 5.19 respectively, according to the radii of the platform and base. The translation vector from the platform to the base ${ }^{B} \vec{q}$ is initialized to $\left[0,0, l_{o}\right]^{T}$ and the pitch-roll-yaw rotation angles are initialized to $\theta=0, \phi=0$ and $\psi=0$. The yaw $\psi$ is always equal to zero as there is never rotation about the $\mathrm{z}$ axis.

b. Next the leg lengths $l_{1}, l_{2}$ and $l_{3}$ and the platform joint positions $\vec{P}_{i}$ are calculated via the inverse kinematics method provided in Section 5.4.1. The platform centre position $O_{P}$ from Figure 5.10 is also computed in the base frame.

c. The algorithm then evaluates the leg lengths $l_{1}, l_{2}$ and $l_{3}$ against the minimum and maximum actuator length constraints $l_{\min }$ and $l_{\max }$. If the leg lengths 


\subsection{KINEMATIC ANALYSIS AND CONTROL SCHEME}

satisfy the $l_{\min }$ and $l_{\max }$ constraints, the verified Cartesian coordinates of points $P_{i}$ and $O_{P}$ are stored. Orientations that do not satisfy the leg length constraints are flagged for further analysis.

d. The algorithm iterates to the next platform orientation and repeats steps $b$ and c until all possible platform orientations have been stepped through.

e. Finally, a 3D plot is generated from the stored points $P_{i}$ and $O_{P}$.

The algorithm was implemented in Matlab where heave was iterated over the interval $l_{o}<q_{z}<\left(l_{o}+200 \mathrm{~mm}\right)$ at a $1 \mathrm{~mm}$ step size and roll and pitch were iterated over the intervals $-20^{\circ}<\phi<20^{\circ}$ and $-20^{\circ}<\theta<20^{\circ}$ respectively at $1^{\circ}$ intervals.

Figure 5.11 plots the workspace of the heave motion platform for the platform joint positions $P_{1}, P_{2}, P_{3}$ and centre point $O_{P}$. The x-y origin is located at the origin of the base platform. Since the platform hangs below the base, all heave values are negative relative to the base. For the purposes of improved visualization, the points in Figure 5.11 are plotted for platform orientations computed at $20 \mathrm{~mm}$ and $10^{\circ}$ intervals instead of the $1 \mathrm{~mm}$ and $1^{\circ}$ step sizes specified above.

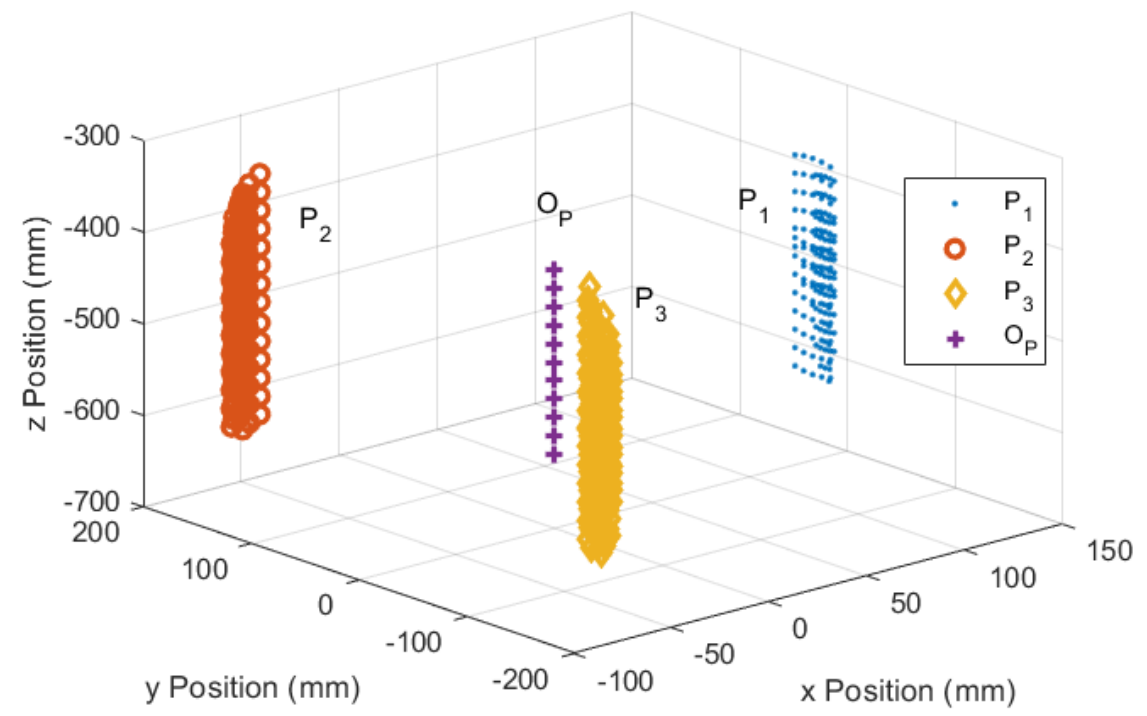

Figure 5.11: Workspace of the surface points $P_{1}, P_{2}, P_{3}$ and origin $O_{P}$. 
CHAPTER 5. DEVELOPMENT AND QUANTIFICATION OF A WAVE GENERATOR AND SHIP MOTION SIMULATOR

Figures 5.12a,b,c plot the workspace of the heave motion platform in the base frame Cartesian planes $\mathrm{x}-\mathrm{y}, \mathrm{x}-\mathrm{z}$ and $\mathrm{y}-\mathrm{z}$ respectively. It is observed in Figure $5.12 \mathrm{a}$ and $5.12 \mathrm{~b}$, that the point $O_{P}$ has no x-y displacement, fulfilling the requirement for pure rotation about the $\mathrm{x}$ and $\mathrm{y}$ axes of the surge-sway gantry system. The $\mathrm{x}$ and y displacement of the platform points $P_{1}, P_{2}, P_{3}$ are due to the roll and pitch of the platform that causes the actuators to rotate at small angles about the pinned joints at the base.

To better visualize the physical manifestation of the inverse kinematics applied to the heave motion platform, the 3D assembly model of the motion platform, shown in Figure 5.9, was imported into the Matlab Simulink environment where the proper constraints were applied to the joints. The driving prismatic joints were given the leg length inputs, computed via the inverse kinematics, to simulate various platform orientations. Figure 5.13 shows the motion platform driven to a position of 100 $\mathrm{mm}$ heave and $20^{\circ}$ roll. Likewise, Figure 5.14 shows the motion platform driven to a position of $100 \mathrm{~mm}$ heave and $20^{\circ}$ pitch. Additionally, Figures 5.13 and 5.14 highlight that the pivot point of each universal joint is actually located at an offset distance from the platform. With this offset, the platform origin will not stay in line with the origin of the base, as observed in workspace plotted in Figures 5.11 and 5.12. This observation prompted a new study of the platform kinematics in order to revise the model to reflect the effects of the universal joint offset and to investigate the significance of the offset distance.

Figure 5.15 illustrates the new geometry that arises from the universal joint offset distance $k$. For the universal joints selected for the heave motion platform the offset $k$ is to be $50.82 \mathrm{~mm}$ from the manufacturer's drawings. The U-joint positions in the base frame ${ }^{B} \vec{U}_{i}$ are defined at the pivot point of the U-joints. Since the distance $k$ was not taken into account in the previous analysis, the $\mathrm{U}$-joint positions ${ }^{B} \vec{U}_{i}$ are in fact the positions previously defined as $P_{i}$ in Equation 5.22. With ${ }^{B} \vec{U}_{i}$ known from the previous analysis, the corrected platform position vectors $\vec{P}_{i}$ must be evaluated. 


\subsection{KINEMATIC ANALYSIS AND CONTROL SCHEME}

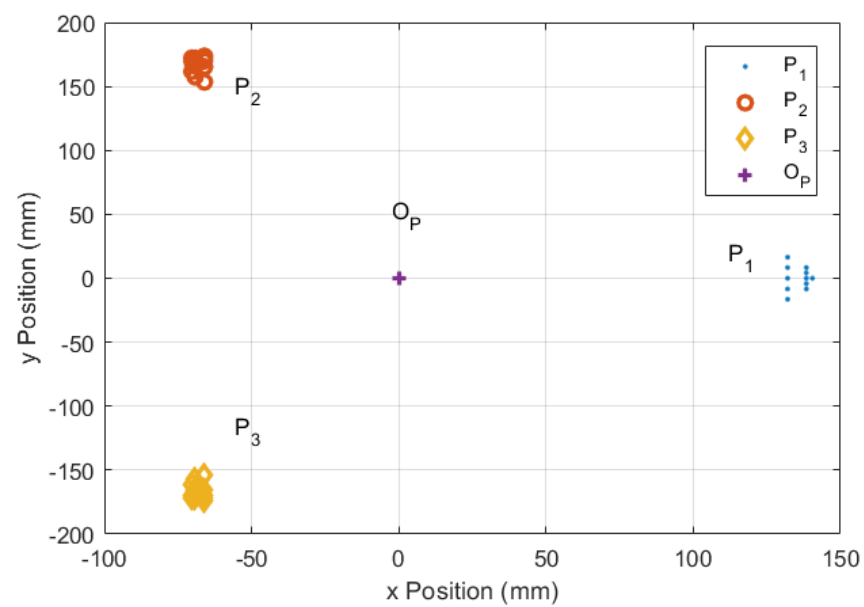

(a)

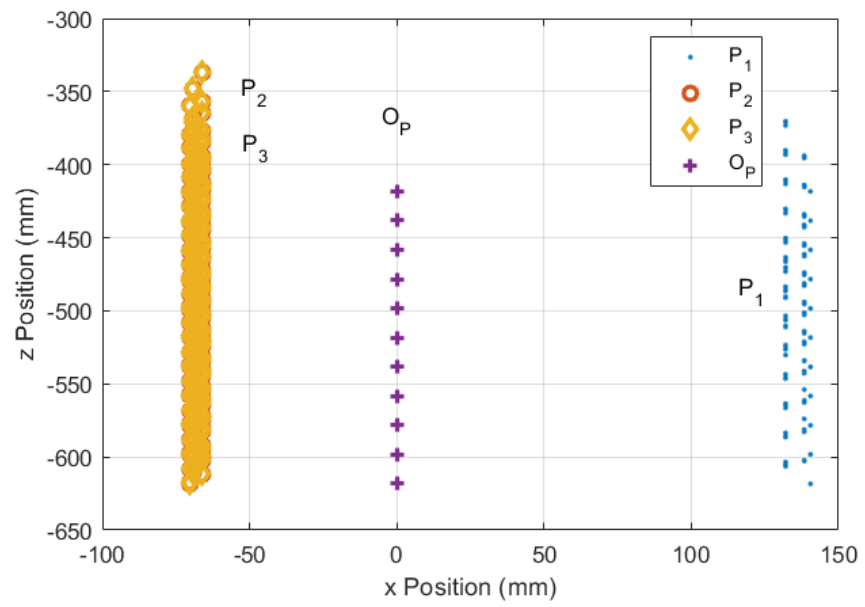

(b)

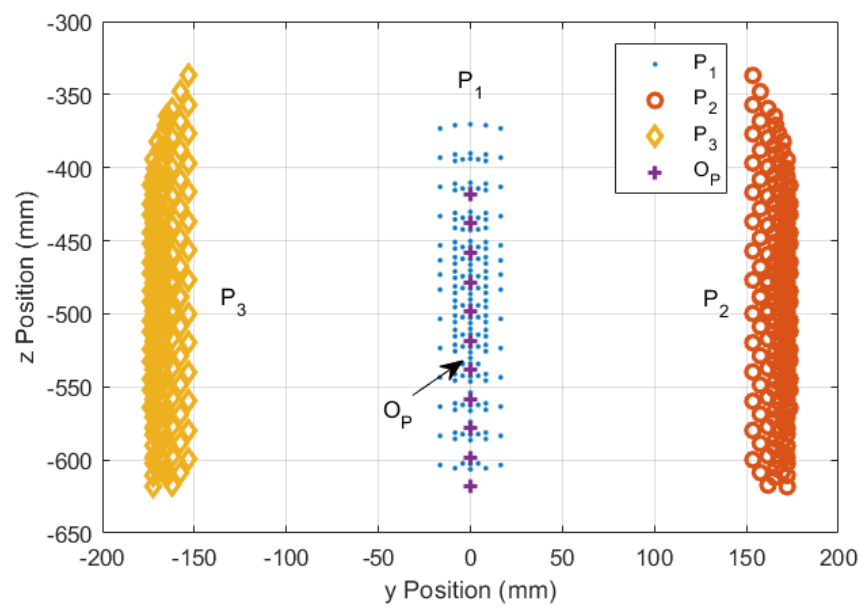

(c)

Figure 5.12: Workspace of the surface points $P_{1}, P_{2}, P_{3}$ and origin $O_{P}$ in the base frame Cartesian planes a)x-y, b)x-z and c)y-z. 

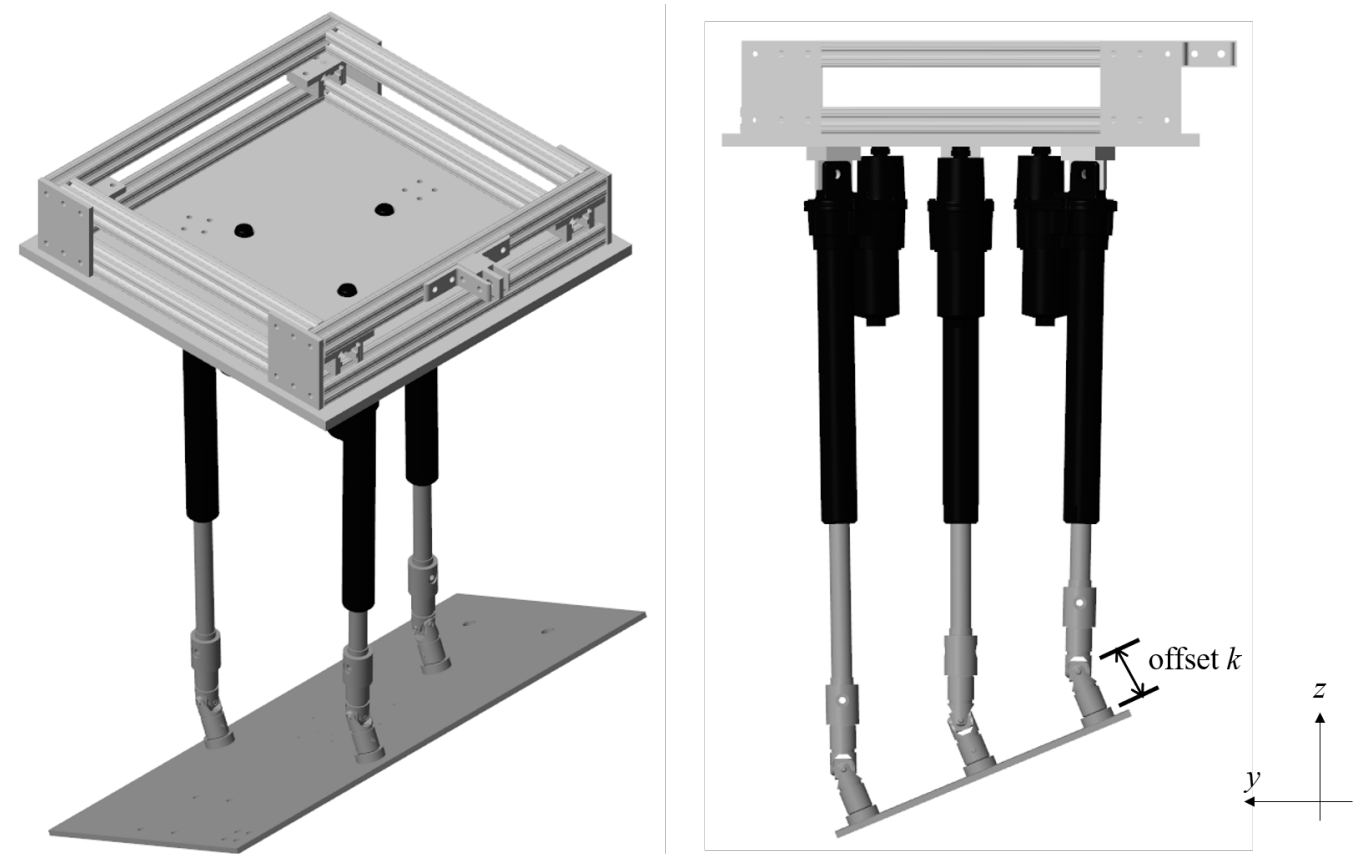

Figure 5.13: Representations of a 20 deg roll angle on the 3-DOF motion platform.

Based on the physical constraints of the fixed end of the universal-joint, the offset $k$ is always perpendicular to the platform and its direction is therefore parallel to the normal vector of the platform. As the position of the platform points expressed in the platform frame have not changed, the normal vector of the plane, $\vec{n}_{p}$ created by the platform can be calculated by

$$
\vec{n}_{p}=\left(\overrightarrow{p_{1}}-\overrightarrow{p_{2}}\right) \times\left(\overrightarrow{p_{1}}-\overrightarrow{p_{3}}\right)
$$

and the magnitude of the normal vector $\hat{n}_{p}$ is

$$
\hat{n}_{p}=\frac{\vec{n}_{p}}{\left\|\vec{n}_{p}\right\|} .
$$

The vector describing the U-joint offset is therefore defined as $k \hat{n}_{p}$. Now, the position of the U-joint pivot point from the base frame ${ }^{B} \vec{U}_{i}$ is known and the position of the U-joint pivot point from the motion platform $k \hat{n}_{p}$ is known. With this information, 


\subsection{KINEMATIC ANALYSIS AND CONTROL SCHEME}
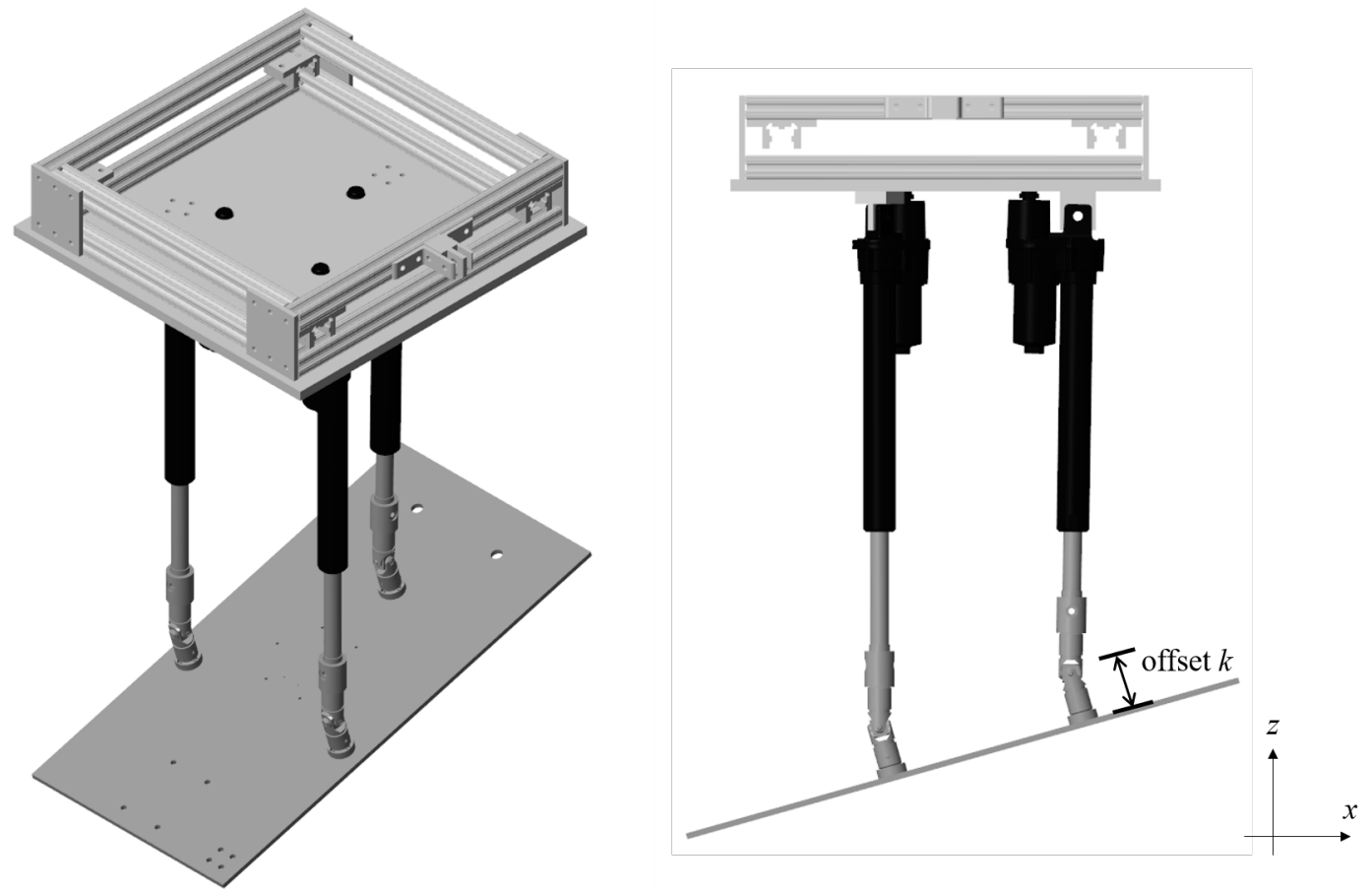

Figure 5.14: Representations of a 20 deg pitch angle on the 3-DOF motion platform.

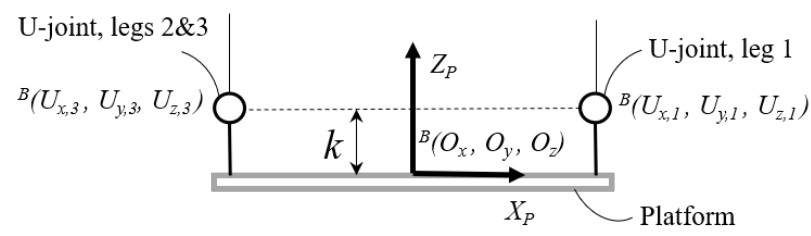

Figure 5.15: U-joint offset $k$ from the platform.

evaluating $\vec{P}_{i}$ now becomes a simple vector addition given by

$$
\vec{P}_{i}={ }^{B} \vec{U}_{i}+k \hat{n}_{p}
$$

Figure 5.16 illustrates the method of evaluating $\vec{P}_{i}$ using the method explained above.

One final correction must be made so that the kinematic model matches the physical motion platform. Since the position $\vec{P}_{i}$ has changed due to the offset, the leg lengths $l_{i}$ must also be amended for proper control and use. Figure 5.17 illustrates the correction that must be made to the leg lengths where the value $l_{i}^{\prime}$ shows the leg lengths that were calculated using the uncorrected inverse kinematics in 


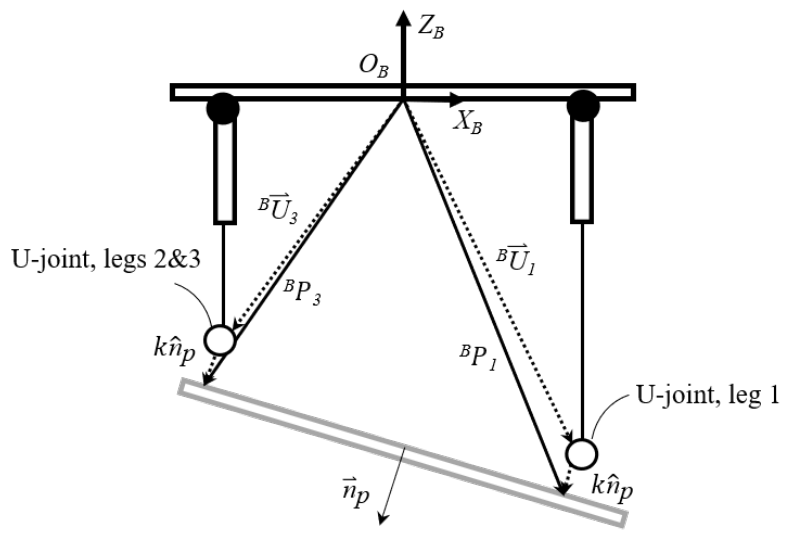

Figure 5.16: Platform position correction due to offset $k$.

the figure. The U-joint pivot point is now displaced above the platform and therefore the correction factor $b$ must be subtracted from $l_{i}^{\prime}$ to obtain the corrected leg lengths $l_{i}$. Defined geometrically, $b$ represents the component of the offset vector $k \hat{n}_{p}$ in the direction of the leg orientation denoted $\hat{l}_{i}$. Now, $b$ can be defined algebraically as the scalar projection of the offset vector $k \hat{n}_{p}$ onto the vector $b \hat{l}_{i}$ as expressed by

$$
b=k \hat{n}_{p} \frac{b \hat{l}_{i}}{\left\|b \hat{l}_{i}\right\|} .
$$

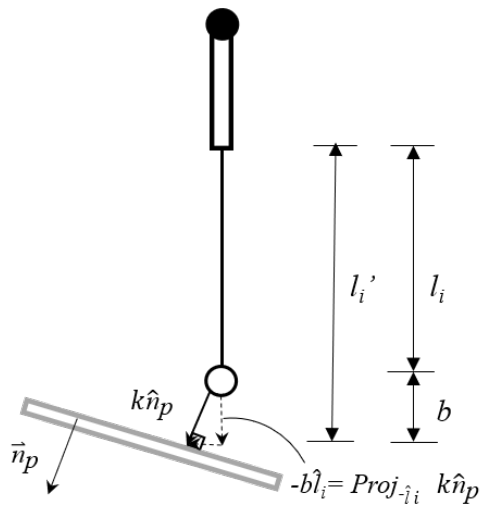

Figure 5.17: Actuator extension correction for the $i^{\text {th }}$ leg.

The heave motion platform workspace was again computed following the procedure outlined above using the corrected platform point positions and leg lengths. Figure 5.18 plots the workspace of the heave motion platform for the platform joint positions $P_{1}, P_{2}, P_{3}$ and centre point $O_{P}$. The x-y origin is located at the origin of 


\subsection{KINEMATIC ANALYSIS AND CONTROL SCHEME}

the base platform. Figures 5.19a,b,c plot the workspace of the heave motion platform in the base frame Cartesian planes $\mathrm{x}-\mathrm{y}, \mathrm{x}-\mathrm{z}$ and $\mathrm{y}-\mathrm{z}$ respectively.

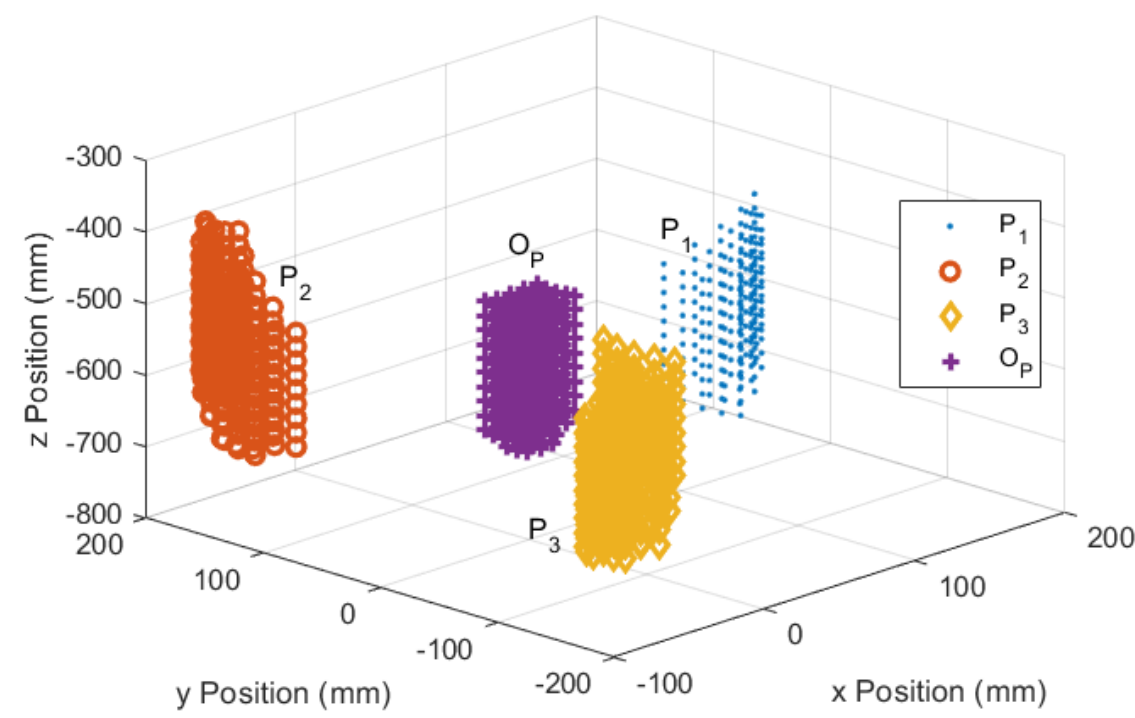

Figure 5.18: Workspace of the surface points $P_{1}, P_{2}, P_{3}$ and origin $O_{P}$ corrected for the U-joint offsets.

It is observed in Figure 5.19a, that the point $O_{P}$ now has displacement in the $\mathrm{x}$ and $\mathrm{y}$ displacements, unlike what was observed in Figure 5.12a. In fact the origin point of the platform $O_{P}$ fluctuates by an amount of $\pm 17.32 \mathrm{~mm}$ in the $\mathrm{x}$ and $\mathrm{y}$. The fluctuation is significant as it means the rotations of the platform are no longer about the base frame $\mathrm{x}$ and $\mathrm{y}$ axes. The movement of the $\mathrm{x}$ and $\mathrm{y}$ platform axes causes the translation relative to the base frame. The translation of the $\mathrm{x}$ and $\mathrm{y}$ axes of the platform frame is separate from the surge and sway translation of the gantry system, or base frame. For small scale ship motion, the additional translation will cause more error if it is not taken into account during trajectory planning.

Figure 5.20 shows the workspace volume of the entire motion platform, relative to the initial platform position of $z=0, \theta=0$ and $\phi=0$. Each plotted dot represents a point along the platform at $5 \mathrm{~cm}$ intervals. The entire clearance volume that must be available to the motion platform during operation can be inferred from Figure 5.20. 
CHAPTER 5. DEVELOPMENT AND QUANTIFICATION OF A WAVE GENERATOR AND SHIP MOTION SIMULATOR

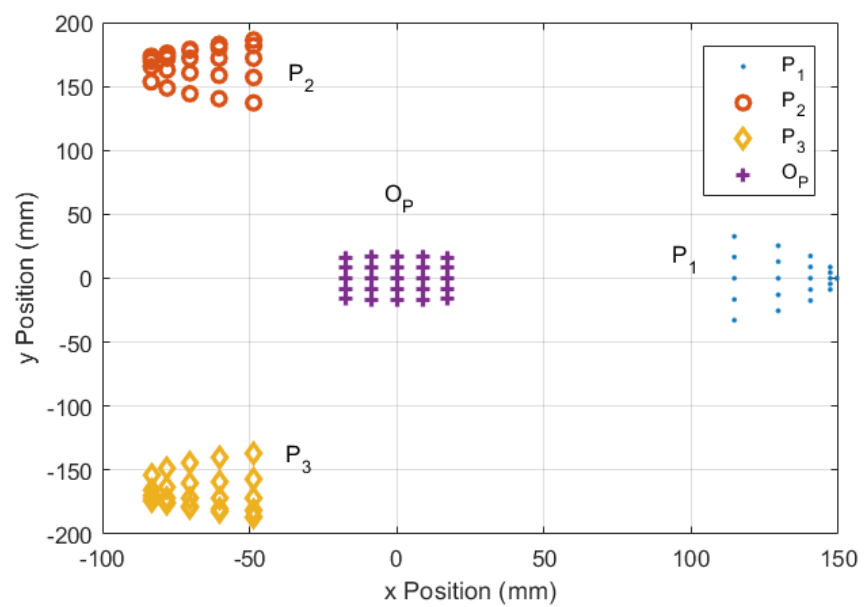

(a)

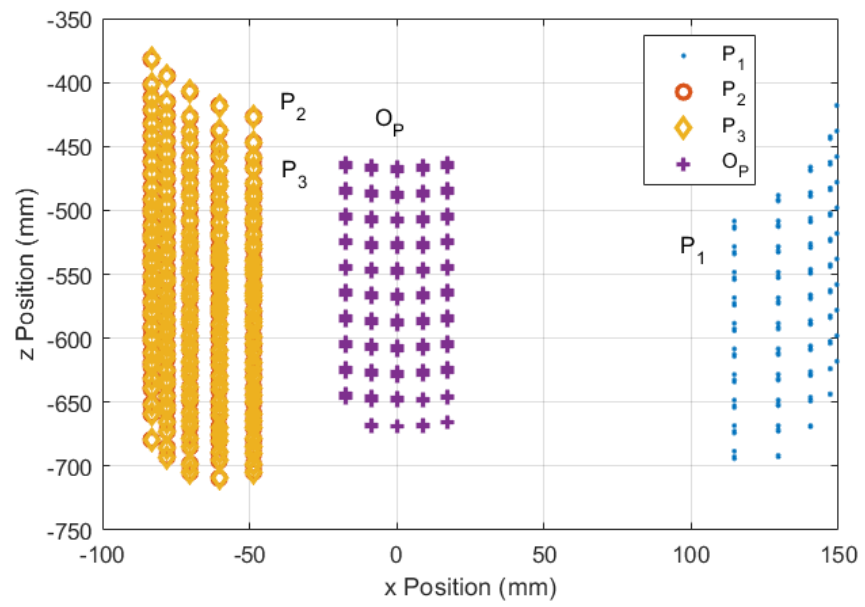

(b)

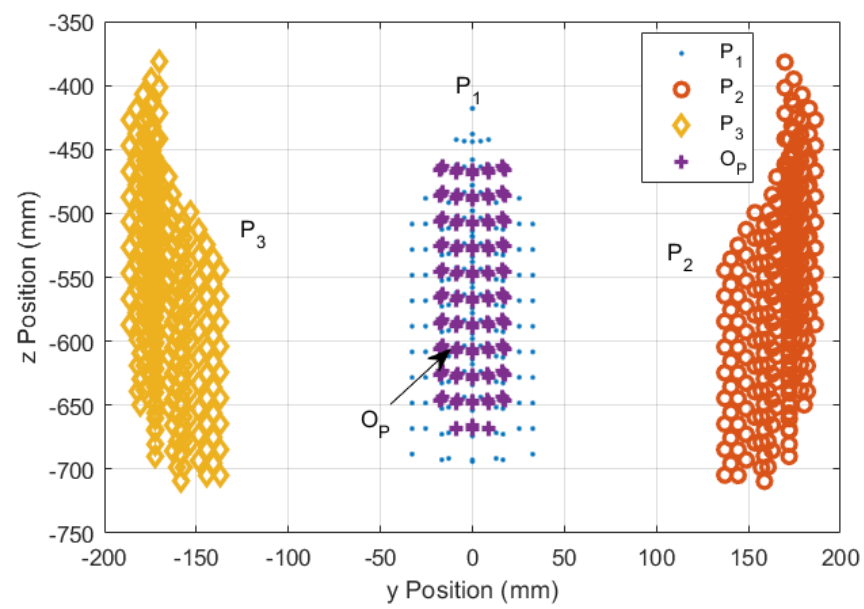

(c)

Figure 5.19: Workspace of the surface points $P_{1}, P_{2}, P_{3}$ and origin $O_{P}$ corrected for the U-joint offsets in the base frame Cartesian planes a)x-y, b) $x-z$ and c)y-z. 


\subsection{KINEMATIC ANALYSIS AND CONTROL SCHEME}

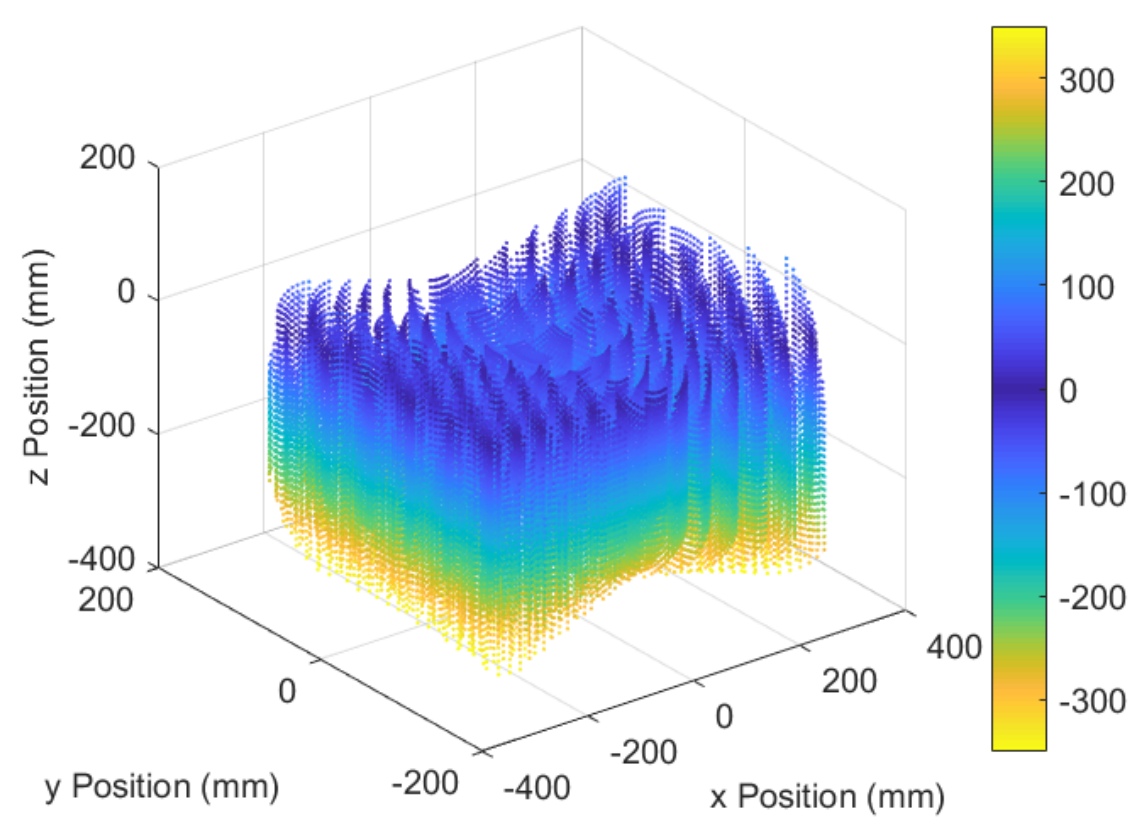

Figure 5.20: Workspace of the entire motion platform, relative to the initial platform position, corrected for the U-joint offsets.

As a final analysis for this section, the flagged positions that did not meet the leg length criterion were investigated to see which platform orientations should be avoided in trajectory planning. The flagged data showed that the positions where the actuator limits were exceeded exclusively occurred when heave was above the relative position of $163 \mathrm{~mm}$ combined with a roll and/or pitch orientation above $0^{\circ}$. Specifically, heave greater than $163 \mathrm{~mm}$ is not possible with any roll or pitch displacement. Therefore, in trajectory planning, heave must always be below the threshold value of $163 \mathrm{~mm}$, relative to $l_{o}$. It is noted that the limitation on heave displacement does not meet the design requirement of a $200 \mathrm{~mm}$ heave displacement but is still considered acceptable. 


\subsection{Preliminary experimental validation of a ship motion simulator system}

The ship motion simulator frame was built out of t-slotted aluminum framing. Actuator mounting brackets, universal joint to actuator adapters and the base and platform

plates were designed and precisely machined in the Carleton University machine shop to required tolerances. The linear actuators selected in the design process have a 500 $\mathrm{N}$ capacity and a speed of $19 \mathrm{~mm} / \mathrm{s}$ fully loaded. Theses actuators exceed the load requirement for a payload of $25 \mathrm{~kg}$, as even if two of three motion platform actuators fail, one actuator can still support the static load of $25 \mathrm{~kg}$ at a safety factor of 2 . In the next section, the control and trajectory planning of the actuator positions is discussed.

\subsubsection{Control and trajectory planning of the ship motion simulator}

Figure 5.21 shows the block diagram of the entire electrical and control system. Position control was selected for the ship motion simulator system. The linear actuators are outfitted with a hall effect sensor that provides analog position feedback for the $200 \mathrm{~mm}$ stroke length, encoded as a $0.5 \mathrm{~V}$ to $4.5 \mathrm{~V}$ signal. To drive the actuators, a linear actuator control board was selected that features a tunable PD position controller. This control board can accept either analog voltage or current signals or digital PWM signals as inputs; however, initial testing of the device showed that the analog input provided the most stable actuator operation compared to a PWM input signal. The entire ship motion simulator system is controlled by the NI myRIO, a real-time embedded module with the required number of analog output ports needed for the control of the five ship motion simulator actuators and one actuator for the wave generator. 


\subsection{PRELIMINARY EXPERIMENTAL VALIDATION OF A SHIP MOTION SIMULATOR SYSTEM}

The PD position controller programmed by the manufacturer into the actuator control board takes the analog feedback signal from an actuator and the desired input position for the actutor from the myRIO, encoded to a $0 \mathrm{~V}-3.3 \mathrm{~V}$ analog input signal, and determines the control signal to be sent to the actuator. However, the actuator control board require a $3.3 \mathrm{~V}-0 \mathrm{~V}$ signal as feedback from the actuators, so a shifter-scaler circuit was built to shift and scale the $0.5 \mathrm{~V}-4.5 \mathrm{~V}$ feedback signal from each actuator to the $0 \mathrm{~V}-3.3 \mathrm{~V}$ scale that can be interpreted by the control board.

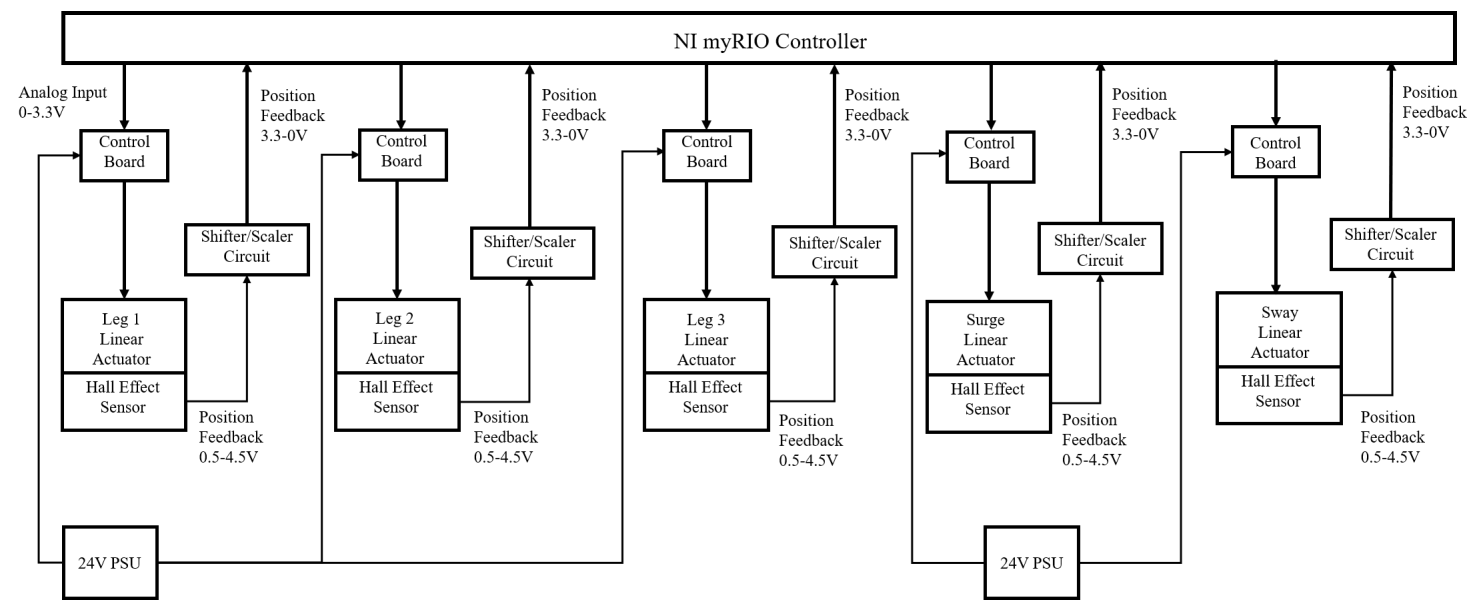

Figure 5.21: Overview of electrical and control system components for the 5-DOF robotic ship motion simulator.

Trajectory planning of the ship motion simulator requires ship motion data. For the preliminary experimental validation of the ship motion simulator, it was desired that the platform be moved through the entire workspace in order to fully test the system. Using ShipMo3D [14], a $120 \mathrm{~m}$ craft was constructed which is representative of a generic frigate. Ship motion was simulated at a speed of $6 \mathrm{kn}$ and at heading of $165^{\circ}$ in an oblique seaway. The seaway was modelled with a unidirectional Bretschneider spectrum where the significant wave height was $5.0 \mathrm{~m}$ and a peak wave period of 12.4 seconds.

The simulated ship motion data was be scaled to the maximum values within the workspace of the ship motion simulator. This scaling was done by reducing the overall maximum value of surge, sway and heave displacements to $200 \mathrm{~mm}$, and 
CHAPTER 5. DEVELOPMENT AND QUANTIFICATION OF A WAVE

GENERATOR AND SHIP MOTION SIMULATOR

checking that the scaled heave displacement did not exceed $160 \mathrm{~mm}$. Next, the maximum value of the roll and pitch positions was scaled to $20^{\circ}$ so that the maximum platform positions could be tested experimentally. Figure 5.22 plots the resulting scaled ship motion data. Yaw was set to $0^{\circ}$ as no yaw motion can be simulated.
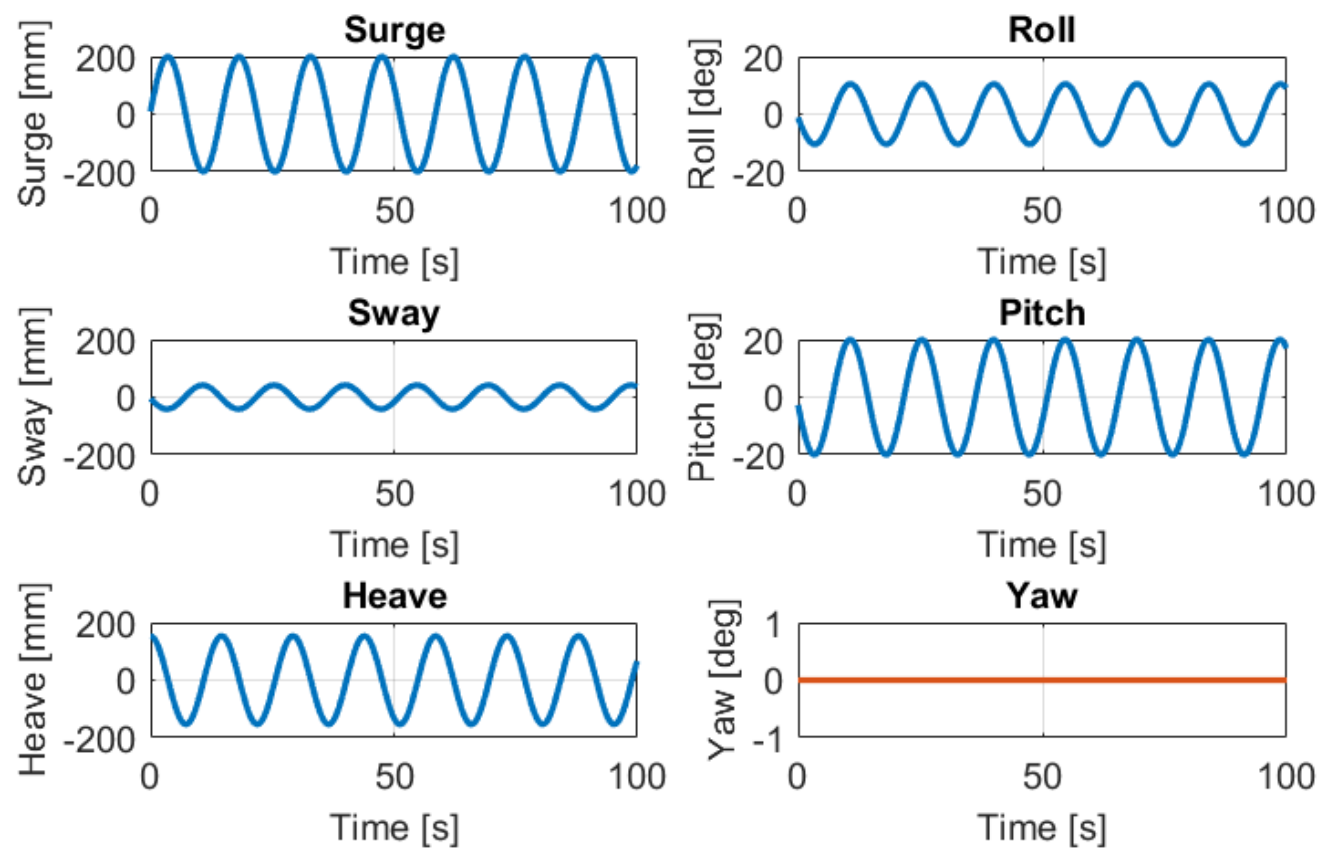

Figure 5.22: Ship motion data in all degrees-of-freedom simulated using ShipMo $3 \mathrm{D}$ and scaled to the workspace of the ship motion simulator.

From the scaled ship motion data, the inverse kinematics of the heave motion platform was use to determine the actuator extension lengths required to achieve the heave-roll-pitch orientations. Next, surge, sway and platform leg displacements were scaled to a 0-3.3 V scale for the analog inputs to the actuator control boards. Tests were first run in the lab to verify the setup, before the robotic ship motion simulator was clamped to the flume tank, as shown in Figure 5.7. 


\subsubsection{Experimental results}

Three experimental tests of the robotic ship motion platform were run for $100 \mathrm{~s}$ of motion. Figure 5.23 compares the actuator setpoint signals and analog feedback signals, in voltage and in $\mathrm{mm}$, as a function of time for each actuator. The actuator feedback signals were flipped in post-processing to a $0 \mathrm{~V}-3.3 \mathrm{~V}$ scale (from the $3.3 \mathrm{~V}$ - $0 \mathrm{~V}$ scale) in order for a direct comparison to be made between the signals. Omitting the first 5 seconds of data where the actuators are observed to be catching up to the setpoint values, the standard deviation of error between the setpoint and feedback signals varied between $0.129 \mathrm{~V}$ and $0.329 \mathrm{~V}(7.8 \mathrm{~mm}$ and $19.9 \mathrm{~mm})$ for the actuators. The maximum error over the same 5-100 s period varied between $0.5 \mathrm{~V}$ and $1.83 \mathrm{~V}$ (30.3 $\mathrm{mm}$ and $111 \mathrm{~mm}$ ) for the actuators. In both cases, the surge actuator yielded the highest standard deviation of error and maximum error.

Most of the error observed between the setpoint and feedback signals is assumed to result from feedback signal lagging the setpoint. This delay is not considered to be an issue with the system because the purpose of the ship motion simulator is to replicate ship motion, which is not a time sensitive application. Furthermore, it is anticipated that an IMU will be mounted to the motion platform in future work that will provide the actual platform orientation in real time. Therefore, from this preliminary analysis, the ship motion simulator system is completed.

A contribution to this thesis was to develop a robotic ship motion simulator to be used in the flume tank laboratory at Carleton University. It was demonstrated the designed system is capable of replicating ship motion using the systems entire workspace. 
CHAPTER 5. DEVELOPMENT AND QUANTIFICATION OF A WAVE

GENERATOR AND SHIP MOTION SIMULATOR

\subsection{Summary}

This chapter has provided an in-depth analysis of the development of key experimental equipment for the Carleton University water channel laboratory, meeting the third and fourth objectives of this thesis. The design of a plunger-type wavemaker has been thoroughly investigated such that the effects of depth, water current and plunger aspect ratios are all quantified. With depth, water current and plunger geometry quantified as design parameters, it was shown that the selection of an operating point provides an objective means to obtain a design solution for specific operating conditions.

The design of the robotic ship motion simulator was also thoroughly analyzed. The kinematic workspace analysis uncovered the previously overlooked parameter of the universal joint offset. It was shown that this offset had a significant effect of the end-effector motion, and that it cannot be ignored. The performance of the ship motion simulator was also investigated through preliminary testing and the system was validated and is ready for use in future experimental testing requiring ship motion. 


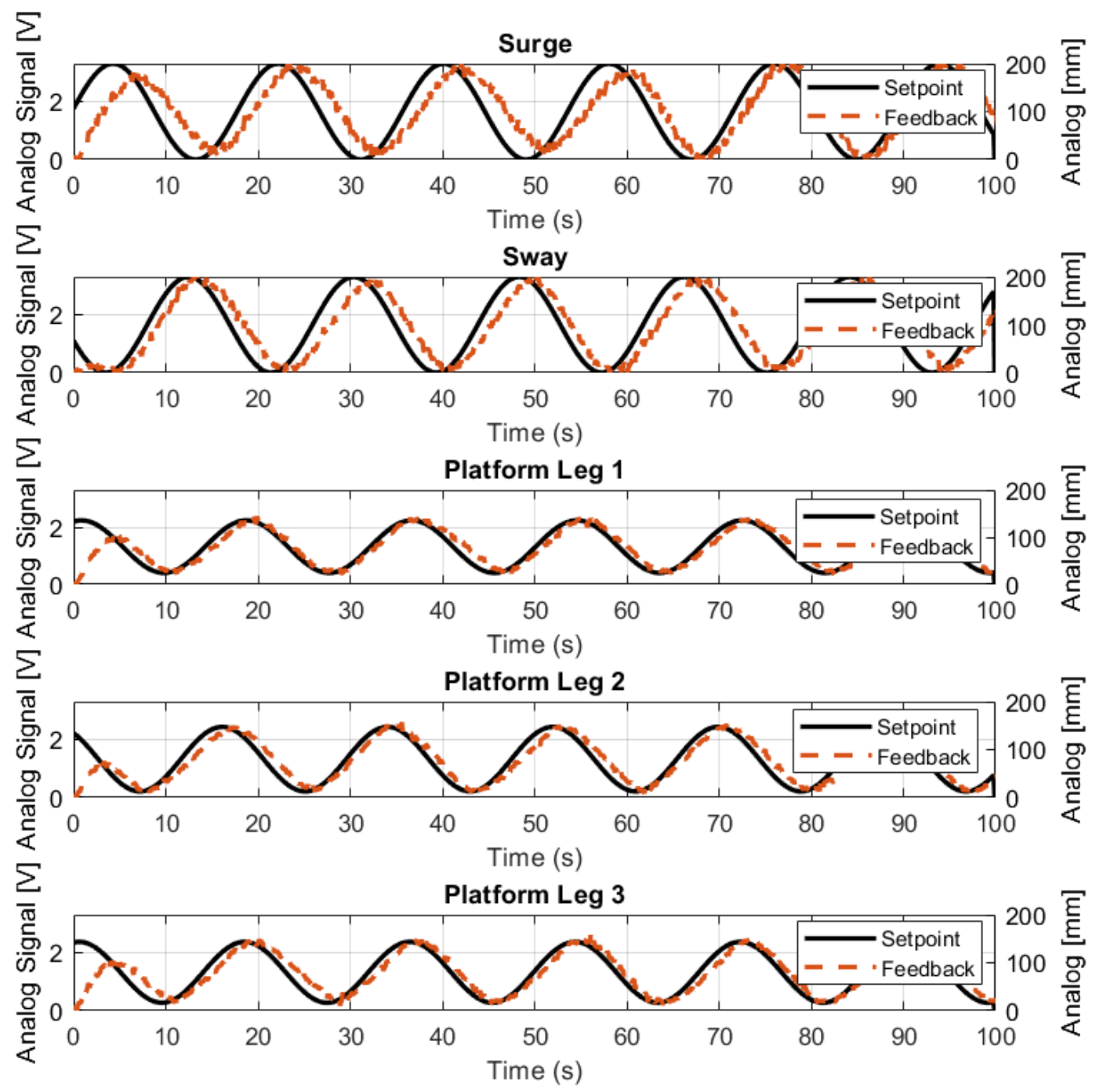

Figure 5.23: Preliminary testing results of the ship motion simulator comparing the analog inputs and actuator feedback signals. 


\section{Chapter 6}

\section{Conclusions and Future Work}

The main objectives of this thesis were:

1. To investigate whether the inclusion of wave synchronization in a set-point algorithm for the control of a winch based compensator system is useful in towed recovery operations.

2. To develop and quantify a robotic ship motion simulator to replicate ship motion in the Carleton University flume tank laboratory.

3. To design a wavemaker capable of creating waves at a flume tank scale.

4. To investigate the fidelity of a wave prediction algorithm for forecasting future breach conditions and present initial development in defining the criteria required for a safe breach condition.

In this chapter, the contributions of the thesis are summarized in the context of the four main objectives in Sections 6.1 - 6.4. Section 6.5 discusses areas of future work relating to this research. 


\subsection{OBJECTIVE 1}

\subsection{Objective 1}

Chapter 3 introduced the effects of waves to Calnan et al.'s cable model, where both regular and irregular waves were modeled. The system was used to simulate a towbody during a lifting operation, where the towbody was reeled in at a constant velocity. The effects of the waves were included in determining the hydrodynamic forces on the towbody both when submerged and during breach where, in the latter, slam loads and exit loads were added.

The simplified waterline and rigorous sheave setpoint algorithms were amended to include the wave motion. The waterline set-point algorithm for heave compensation determines a setpoint for a winch system control loop based on ensuring that the same point along the tow cable always crosses the mean water level. With wave synchronization, the setpoint ensures that the distance from the sheave to the mean water level is kept constant during the lifting operation. The revised setpoint is determined by subtracting the water surface elevation from the height of the sheave above the mean water level. The sheave setpoint algorithm determines the amount of cable to reel in and out based on the projection of the sheave displacement onto its nominal position. To include wave synchronization, the sheave setpoint is amended by including the displacement of the water surface caused by waves with the sheave displacement.

The simulation results indicated that wave synchronization does not benefit compensator strategies based on the metrics used in the analysis. When considering the standard deviation of the cable tension, the metric used by Sagutun et al. [2], the simulations that were run with combined wave synchronization and heave compensation performed similarly to those run with only heave compensation. Moreover,

both the combined and heave only strategies performed worse than simulations with no control method applied in all cases. It is therefore suggested that motion compensation efforts for towed recovery operations do not require the added complexity of wave synchronization. 


\section{CHAPTER 6. CONCLUSIONS AND FUTURE WORK}

\subsection{Objective 2}

In Chapter 5, a robotic ship motion simulator was designed to simulated surge, sway, heave, roll and pitch motion. The design includes a 3-DOF motion platform that reproduces the heave, roll and pitch. A kinematic analysis was performed to characterize the workspace of the system, from which it was shown that a maximum constraint of $160 \mathrm{~mm}$ is required for the heave motion of the end effector. The performance of the ship motion simulator was also investigated through preliminary experimental testing where the standard deviation of error between the desired input ship motion and the resulting recorded actuator motion varied from $7 \mathrm{~mm}$ to $19 \mathrm{~mm}$ between actuators. The surge actuator motion saw the largest standard deviation of error, which was attributed to lag in the system. It is anticipated that this lag can be removed from the feedback signal in post processing.

\subsection{Objective 3}

Also in Chapter 5, wavemaker theory was investigated for plunger type wavemakers: the wavemaker type that was selected for the flume tank environment in Chapter 2. The influence of water depth and plunger aspect ratio were explored through Wu's numerical wavemaker model [24]. The numerical model was advanced to include the effects of water current on plunger type wavemaker designs. It was shown that, with increasing current in the direction of propagating waves, the amplitude to stroke ratio decreases. With the operating conditions fully defined and included in the wavemaker model, the selection of a design operating point was discussed, providing an objective means for the design of a plunger type wavemaker, which previously was not found in the literature. 


\subsection{OBJECTIVE 4}

\subsection{Objective 4}

Chapter 4 described the generalized formulation and tuning of a wave prediction algorithm as it applies to the determination of a Go-NoGo state for predicting safe breach conditions. Key modification made to the SPA were the implementation of continuous mode detection, that enables the algorithm to dynamically handle changing conditions of the input signal, and tuning the SPA by setting a peak height sensitivity parameter that is used for determining the dominant peaks in the FFT spectrum during mode detection. For the normalized amplitudes of both wave and ship motion, setting the peak sensitivity parameter to a value of $\mu=0.1$ was found to be sufficient in simulations.

The chapter also described the development of useful GO criteria used for producing the Go-NoGo state used to identify safe breaching opportunities. Because prediction error increases over the prediction horizon, it was determined that a GO scenario identified by the maximum point of the prediction should not be used, as this approach can lead to erroneous conclusions regarding the Go-NoGo state. Instead, a GO scenario defined by the mean and one standard deviation of the predicted signal over the prediction horizon was found to produce a Go-NoGo signal that agreed most with the desired Go-NoGo signal for up to $10 \mathrm{~s}$.

Performance of the SPA-Go-NoGo function was further improved with the implementation of a latching algorithm that successfully removed undesirable fluctuation in the Go-NoGo signal.

\subsection{Future Work}

This thesis has shown that separate wave synchronization is not required for towed launch and recovery operations in simulation. Signal prediction could be used elsewhere for improving active compensator strategies. The following are recommenda- 


\section{CHAPTER 6. CONCLUSIONS AND FUTURE WORK}

tions for future work that can validate and improve simulation results and build upon this research:

1. Experimental validation of the flume-scale wave synchronization set point simulation results.

2. Experimental quantification of the robotic ship motion simulator using realtime measurements of the end-effector position to validate the kinematic model of the system.

3. Build and test a plunger-type wavemaker based on the operating point selection discussed in Chapter 5. The wavemaker can then be used to verify the addition of current to the numerical wavemaker model. Due to the short length of the flume tank test section, reflected waves are expected to influence the partially standing wave field. The effects of wave reflection should be measured and quantified. Reducing the amount of wave reflection could also be investigated through the use of a passive wave absorber.

4. A more accurate cable model that includes winch and sheave dynamics could be used in simulation, such as that developed by Westin $[53,54,55]$, to improve the wave synchronization simulation results.

5. Perform full scale simulations to verify flume scale simulation results in realworld conditions.

\subsection{List of Publications}

The following papers were published based on the work shown in this thesis:

1. McPhee J., Irani R.A. (2018) On-line Determination of a Go-NoGo State Using a Continous Estimation of the System Response, In the Proceedings of 


\subsection{LIST OF PUBLICATIONS}

The Canadian Society for Mechanical Engineering International Congress 2018 (CSME 2018), May 27-30, 2018, Toronto, ON, Canada.

2. Abujoub S., McPhee J., Westin C., Irani R. A. (2018) Unmanned Aerial Vehicle Landing on Maritime Vessels using Signal Prediction of the Ship Motion, OCEANS 2018 Charleston Conference October 22 - 25, 2018. 


\section{References}

[1] Clark Calnan. Set-point algorithms for active heave compensation of towed bodies. Master's thesis, Dalhousie University, Dalhousie, Nova Scotia, 2016.

[2] S. I. Sagutun, T. A. Johansen, T. I. Fossen, and F. G. Nielsen. Wave synchronizing crane control during water entry in offshore moonpool operations. In Proceedings IEEE Conference Control Applications, Glasgow, Scotland, 2002.

[3] J.K. Woodacre, R.J. Bauer, and R.A. Irani. Hydraulic valve-based active-heave compensation using a model-predictive controller with non-linear valve compensations. Ocean Engineering, 152:47-56, 2018.

[4] Jeffrey Woodacre. Model predictive control of a hydraulic active heave compensation system with heave prediction. Master's thesis, Dalhousie University, Halifax, 2015.

[5] Tor A. Johansen, Thor I. Fossen, Svein I. Sagatun, and Finn G. Nielsen. Wave synchronizing crane control during water entry in offshore moonpool operations - experimental results. IEEE Journal of Oceanic Engineering, 28:720-728, 2003.

[6] S. B. van Albada, G. D. van Albada, H. P. Hildre, and H. Zhang. A novel approach to anti-sway control for marine shipboard cranes. In 27th European Conference on Modelling and Simulation, 2013. 


\section{REFERENCES}

[7] Frode Nymark Eikeland. Compensation of wave-induced motion for marine crane operations. Master's thesis, Norwegian University of Science and Technology, Trondheim, Norway, 2008.

[8] Rolling Hill Research Corporation. Research Water Tunnels Specifications. Rolling Hill Research Corporation.

[9] Robert G. Dean and Robert A. Dalrymple. Water Wave Mechanics for Engineers and Scientists. Prentice-Hall, 1984.

[10] J.Longo and F.Stern. Yaw effects on model-scale ship flows. In Twenty-First Symposium on Naval Hydrodynamics, 1997.

[11] O. M. Faltinsen. Sea Loads on Ships and Offshore Structures. Cambridge University Press, 1990.

[12] Subrata K. Chakrabarti. Handbook of Offshore Engineering, Volumes 1-2 - 3.6.2 Applicability of Spectrum Model. Elsevier, 2005.

[13] Robert H. Stewart. Introduction To Physical Oceanography. Texas A\&M University, 2008.

[14] Kevin McTaggart. ShipMo3D version 3.0 user manual for creating ship models. Tech. Rep. TM 2011-308, Defence Research and Development Canada, 2012, 2012.

[15] ITTC seakeeping committee report. Technical report, ITTC Seakeeping Committee, The Hague, 1978.

[16] Beach management manual. Technical report, CIRIA, 1996.

[17] Mohammad Janvad Ketabdari and Ali Ranginkaman. Numerical simulation of random irregular waves for wave generation in laboratory flumes. Amirkabir International Journal Of Modeling, Identification, Simulation And Control, 43:1$6,2011$. 


\section{REFERENCES}

[18] NATO. NATO - STANAG 4194 Standardized Wave and Wind Environments and Shipboard Reporting of Sea Conditions. North Atlantic Treaty Organization, 1983.

[19] A. Arney. FFG-7 ship motion and airwake trial. part II: removal of ship motion effects from measured airwake data. Air Operations Division Aeronautical and Maritime Research Laboratory, 1994.

[20] F. Biesel and F. Suquet. Laboratory wave generating apparatus. University of Minnesota, 1954.

[21] Eric R. Chappell. Theory and design of a wave generator for a short flume. Master's thesis, University of British Columbia, Vancouver, British Columbia, 1969.

[22] Flap ocean wavemakers. http://www4.edesign.co.uk/product/ocean-flap-wavegenerator/. Accessed: 2019-07-09.

[23] Shen Wang. Plunger type wavemakers: Theory and experiment. Journal of Hydraulic Research, 12(3):357-388, 1974.

[24] Yung-Chao Wu. Plunger-type wavemaker theory. Journal of Hydraulic Research, 26(4):483-491, 1989.

[25] J. N. Newman. Marine Hydrodynamics. MIT Press, Cambridge, MA, 1977.

[26] Det Norske Veritas. Recommended Practice DNV-RP-H103: Modelling and Analysis of Marine Operations. Det Norske Veritas, 2011.

[27] M. Greenhow and L. Yanbao. Added masses for circular cylinders near or penetrating fluid boundaries - review, extension and application to water-entry, -exit and slamming. IEEE Journal of Oceanic Engineering, OE-14:325-348, 1987.

[28] Thor I. Fossen and Tor Arne Johansen. Modeling and identification of an offshore crane-rig system. Norwegian University of Science and Technology, 2001. 


\section{REFERENCES}

[29] Clark Calnan, Robert J. Bauer, and Rishad A. Irani. Reference-point algorithms for active motion compensation of towed bodies. IEEE Journal of Oceanic Engineering, pages 1-17, 2018.

[30] C. Calnan, R. Bauer, and R. A. Irani. Controller design and generalized motion compensation for marine towed bodies. In Oceans16 MTS IEEE Montery, Montery, USA, 2016.

[31] F. Hover, M. Grosenbaugh, and M. Triantafyllou. Calculation of dynamic motions and tensions in towed underwater cables. IEEE Journal of Oceanic Engineering, 9:449-457, 1994.

[32] Driscoll, Lueck, and Nahon. Development and validation of a lumped-mass dynamics model of a deep-sea rov system. Applied Ocean Research, 22:169-182, 2000.

[33] J. Kamman and W. Huston. Multibody dynamics modeling of variable length cable systems. Multibody System Dynamics, 5:211-221, 2001.

[34] B. Mutlu Sumer and Jørgen Fredsøe. Hydrodynamics Around Cylindrical Structures. World Scientific, Singapore, 1997.

[35] Eric W. Weisstein. Circular segment. MathWorld-A Wolfram Web Resource, 2019.

[36] Frank M. White. Fluid Mechanics. McGraw Hill, New York, NY, 3 edition, 2009.

[37] J. McPhee and R. A. Irani. On-line determination of a go-nogo state using a continuous estimation of the system response. In Proceedings of The Canadian Society of Mechanical Engineering International Congress 2018 (CSME 2018), Toronto, Canada, 2018.

[38] S. Abujoub, J. McPhee, C. Westin, and R. A. Irani. Unmanned aerial vehicle landing on maritime vessels using signal prediction of the ship motion. In OCEANS 2018 MTS/IEEE Charleston, Charleston, USA, 2018. 


\section{REFERENCES}

[39] Gabriel David Weymouth, Robert Vance Wilson, and Frederick Stern. Rans computational fluid dynamics predictions of pitch and heave ship motions in head seas. Journal of Ship Research, 49:80-97, 2005.

[40] S. Kuchler, T. Mahl, J. Neupert, K. Schneider, and O. Sawodny. Active control for an offshore crane using prediction of the vessels motion. Mechatronics, IEEE/ASME Transactions on, 16:297-309, 2011.

[41] J. Woodacre, W. Wahidi, R. Bauer, and R. Irani. Coupling a standard hydraulic valve and advanced control to achieve a motion compensation system. In $A S N E$ Launch and Recovery Symposium, 2016.

[42] Rastislav Telgársky. Dominant frequency extraction. Central New Mexico Community College, 2013.

[43] T. H. Havelock. Forced surface-waves on water. The London, Edinburgh, and Dublin Philosophical Magazine and Journal of Science, 8(51):569-576, 1929.

[44] C. J. Galvin Jr. Wave-height prediction for wave generators in shallow water. Tech. Memo 4, U.S. Army, Coastal Engineering Research Center, 1964.

[45] F. Ursell. On the heaving motion of a circular cylinder on the surface of a fluid. The Quarterly Journal of Mechanics and Applied Mathematics, 2(2):218-231, 1949 .

[46] R. D. Russell and L. F. Shampine. A collocation method for boundary value problems. L.F. Numer. Math., 19(1):483-491, 1972.

[47] Marili Viitak, Ilja Maljutenko, Victor Alari, Ulo Suursaar, Sander Rikka, and Priidik Lagemaa. The impact of surface currents and sea level on the wave field evolution during st. jude storm in the eastern baltic sea. Oceanlogia, 58(3):176$186,2016$.

[48] D. Menemenlis C.B Rocha N. Rascle B. Chapron J. Gula J. Molemaker F. Ardhuin, ST Gille. Small-scale open ocean currents have large effects on wind wave heights. Journal of Geophysical Research-Oceans, 122:4500-4517, 2017. 


\section{REFERENCES}

[49] Kai Liu, John M. Fitxgerald, and Frank L. Lewis. Kinematic analysis of a stewart platform manipulator. IEEE TRANSACTIONS ON INDUSTRIAL ELECTRONICS, 40(2):282-293, 1993.

[50] C. C. Ng, S. K. Ong, and A. Y. C. Nee. Design and development of 3-dof modular micro parallel kinematic manipulator. The International Journal of Advanced Manufacturing Technology, 31(1-2):188-200, 2006.

[51] Derek K. Brecht. A 3-dof stewart platform for trenchless pipeline rehabilitation. Master's thesis, University of Western Ontario, London, Ontario, 2015.

[52] Mircea Badescu, Jeremy Morman, and Constantinos Mavroidis. Workspace optimization of orientational 3-legged ups parallel platforms. In Proceedings of DETC'02 ASME 2002 Design Engineering Technical Conferences and Computer and Information in Engineering Conference, Montreal, Canada, 2002.

[53] Cassidy Westin. Modelling and simulation of marine cables with dynamic winch and sheave contact. Master's thesis, Carleton University, Ottawa, Ontario, 2018.

[54] C. Westin and R. A. Irani. Vortex-induced vibrations of a low-tension cablesheave system modeled using nonlinear finite elements. In In the Proceedings of The Canadian Society for Mechanical Engineering International Congress 2018, Toronto, Canada, 2018.

[55] C. Westin and R. A. Irani. Cable-pulley interaction with dynamic wrap angle using the absolute nodal coordinate formulation. In In the proceedings of the 4 th International Conference of Control, Dynamic Systems, And Robotics, Toronto, Canada, 2017. 
Appendix A

Additional Figures 


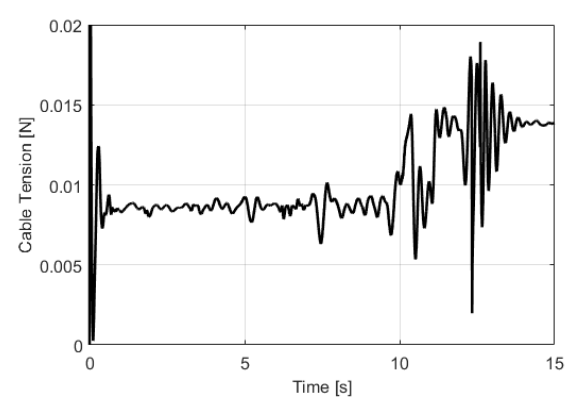

(a) Combined heave compensation and wave synchronization.

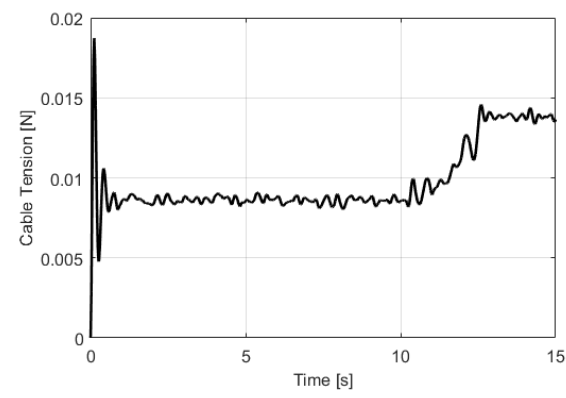

(c) Heave compensation.

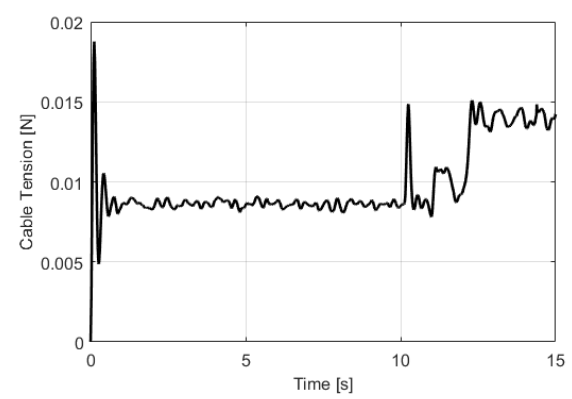

(e) Null control.

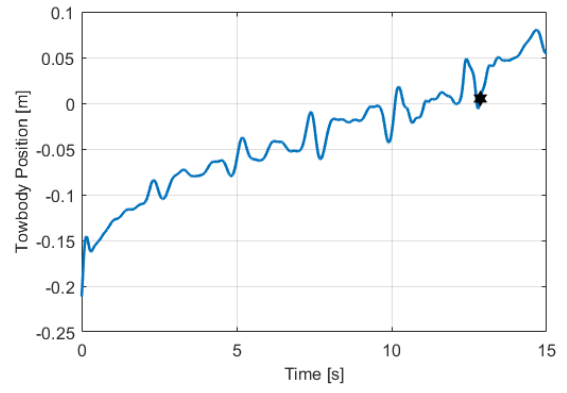

(b) Combined heave compensation and wave synchronization.

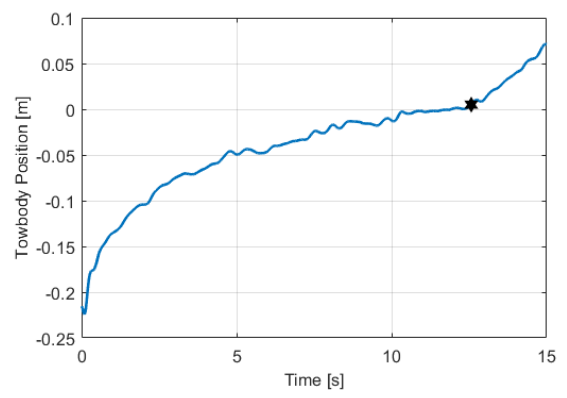

(d) Heave compensation

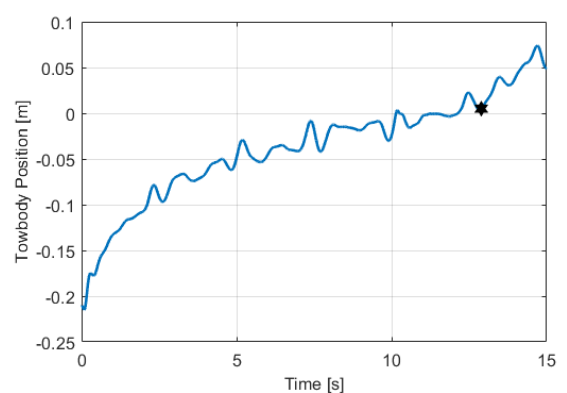

(f) Null Control

Figure A.1: Simulated results with irregular waves at SS6, $T_{p}=1.01 \mathrm{~s}$ and $H_{s}=$ $0.035 \mathrm{~m}$. Wire tension is shown on the left for a) combined heave compensation and wave synchronization, c) heave compensation only and e) no control. The towed body position in the world frame $z$ direction is shown on the right for b) combined heave compensation and wave synchronization, d) heave compensation only and f) no control. The towed body breach point is indicated by a hexagram in the towed body position figures. 


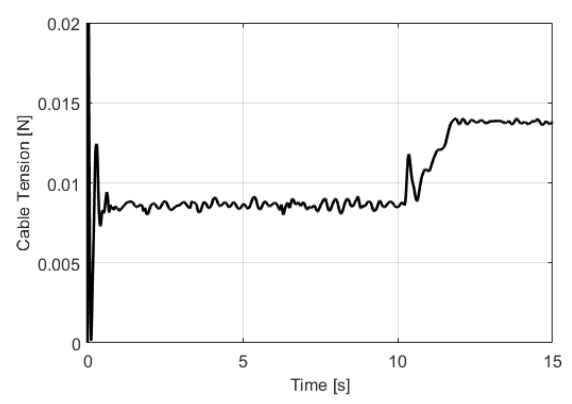

(a) Combined heave compensation and wave synchronization.

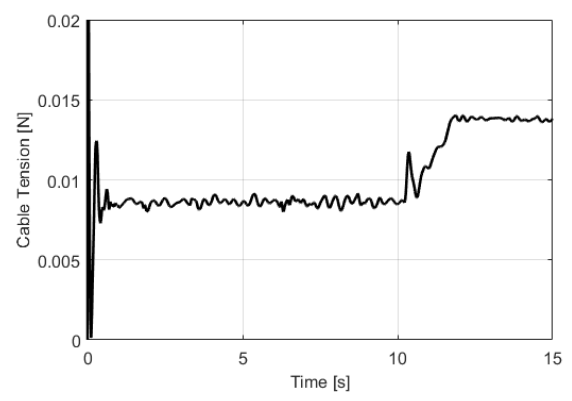

(c) Heave compensation.

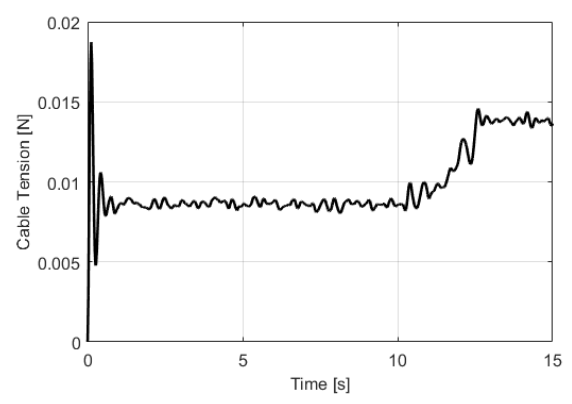

(e) Null control.

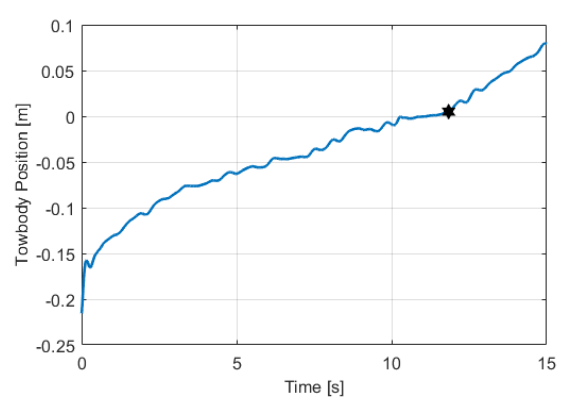

(b) Combined heave compensation and wave synchronization.

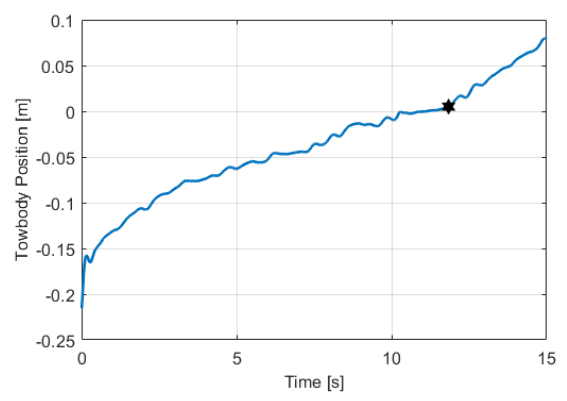

(d) Heave compensation

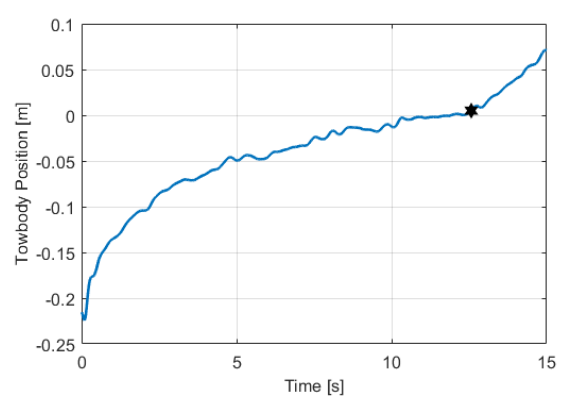

(f) Null Control

Figure A.2: Simulated results with irregular waves at SS3, $T_{p}=0.63 \mathrm{~s}$ and $H_{s}=$ $0.007 \mathrm{~m}$. Wire tension is shown on the left for a) combined heave compensation and wave synchronization, c) heave compensation only and e) no control. The towed body position in the world frame $z$ direction is shown on the right for b) combined heave compensation and wave synchronization, d) heave compensation only and f) no control. The towed body breach point is indicated by a hexagram in the towed body position figures. 

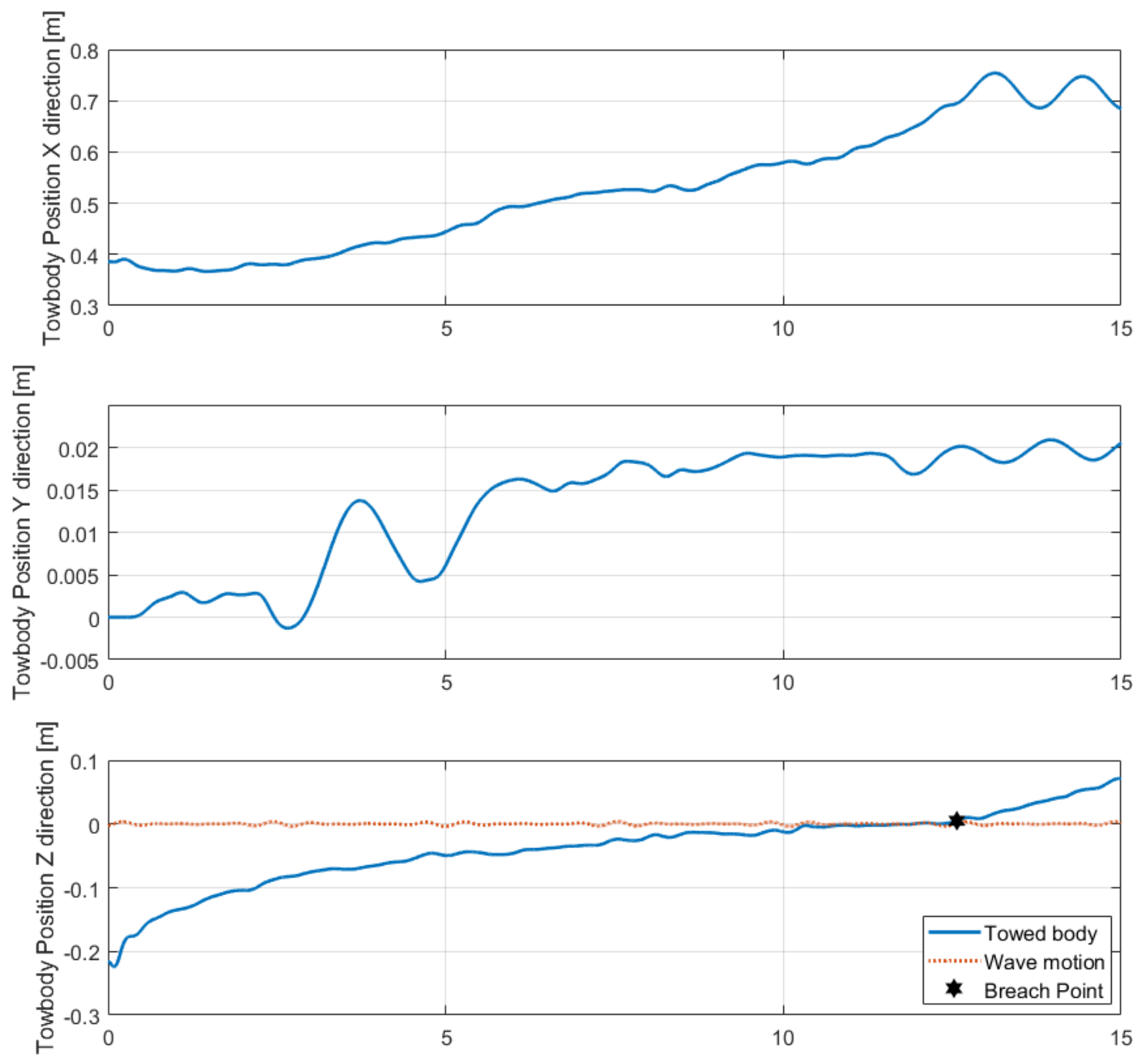

Figure A.3: Simulated results for the towed body position with no control during a recovery operation with irregular waves at SS3. The towed body breach point is indicated by a hexagram. 


\section{APPENDIX A. ADDITIONAL FIGURES}
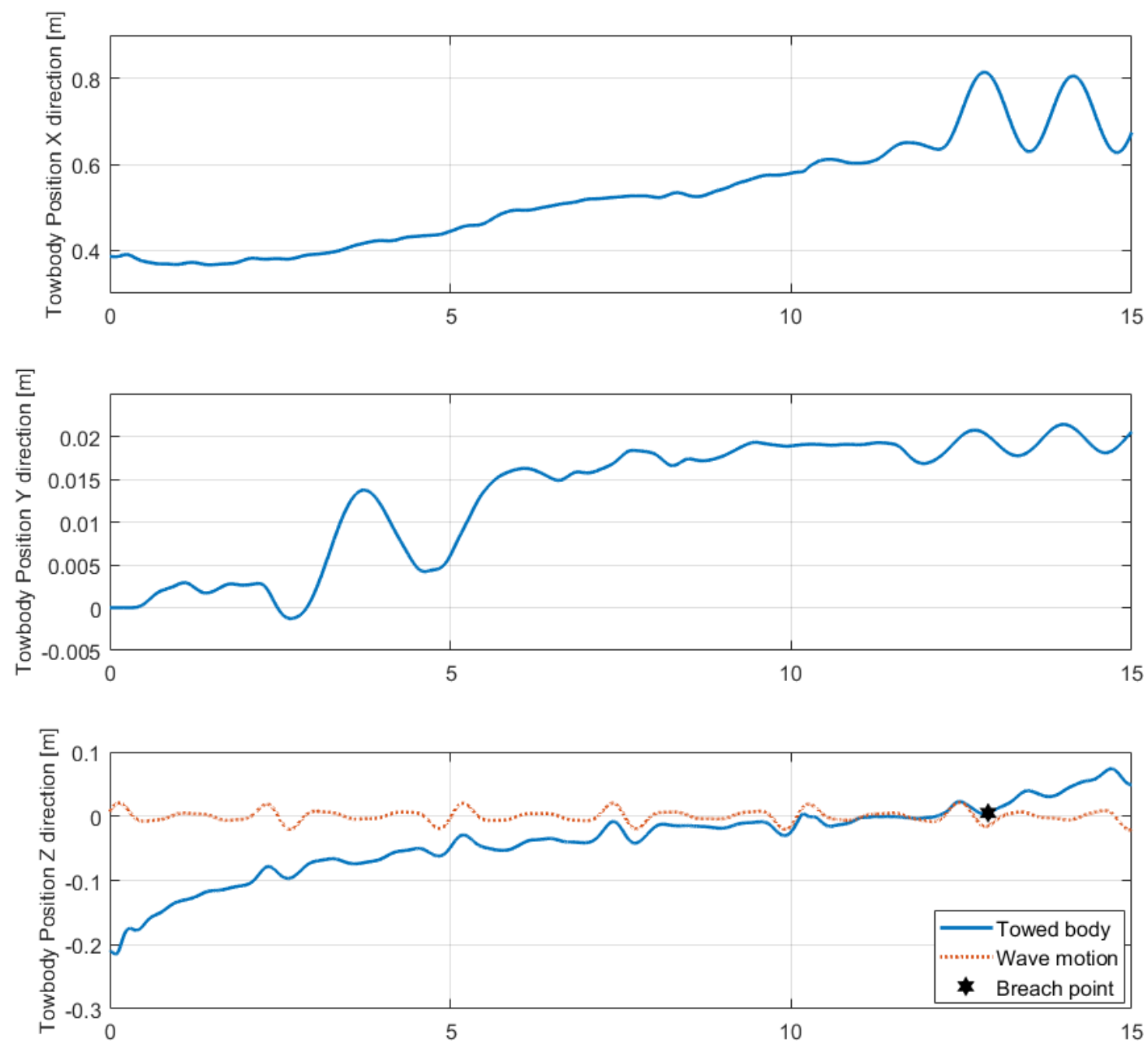

Figure A.4: Simulated results for the towed body position with no control during a recovery operation with irregular waves at SS6. The towed body breach point is indicated by a hexagram. 

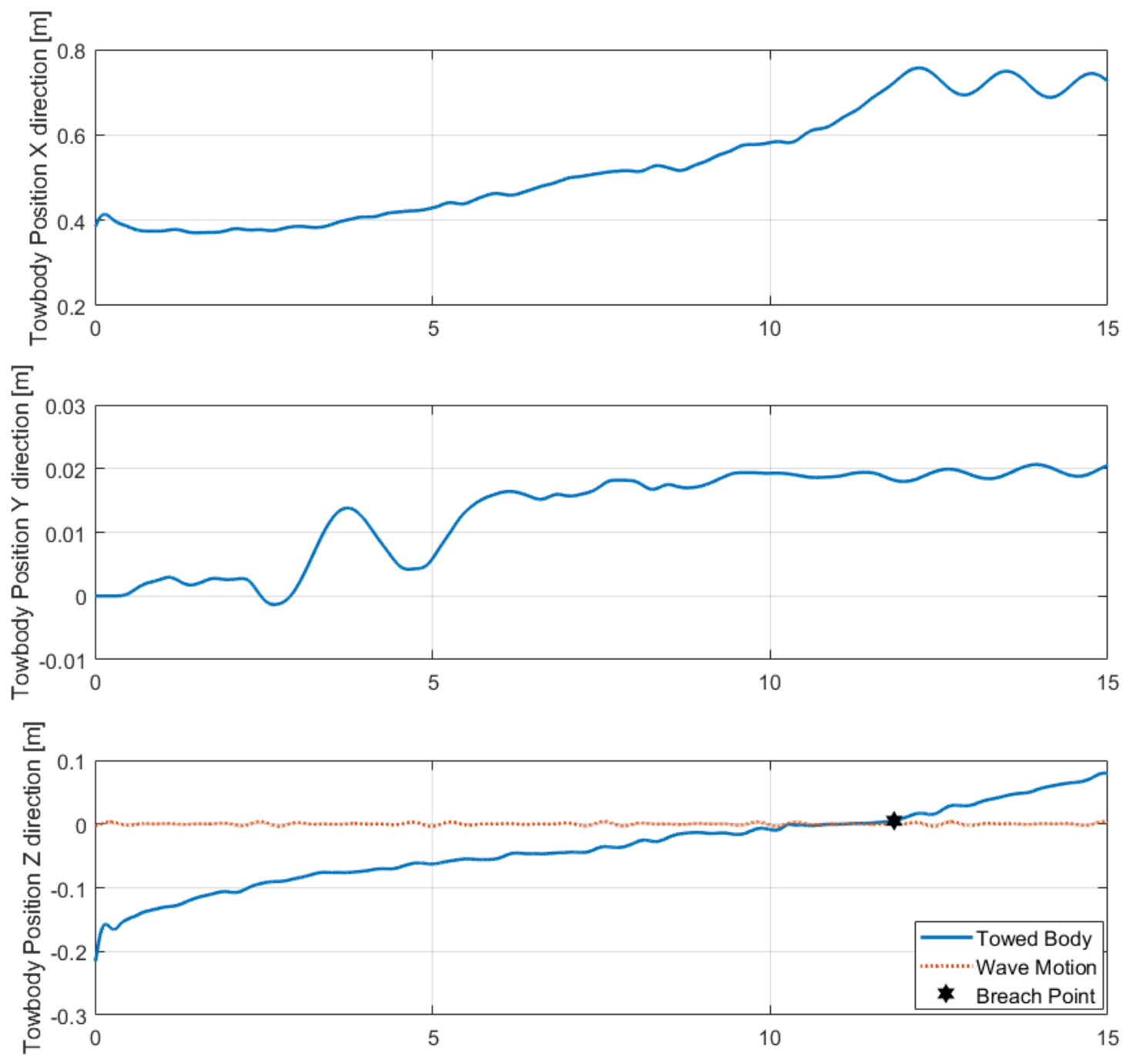

Figure A.5: Simulated results for the towed body position with only heave compensation during a recovery operation with irregular waves at SS3. The towed body breach point is indicated by a hexagram. 


\section{APPENDIX A. ADDITIONAL FIGURES}
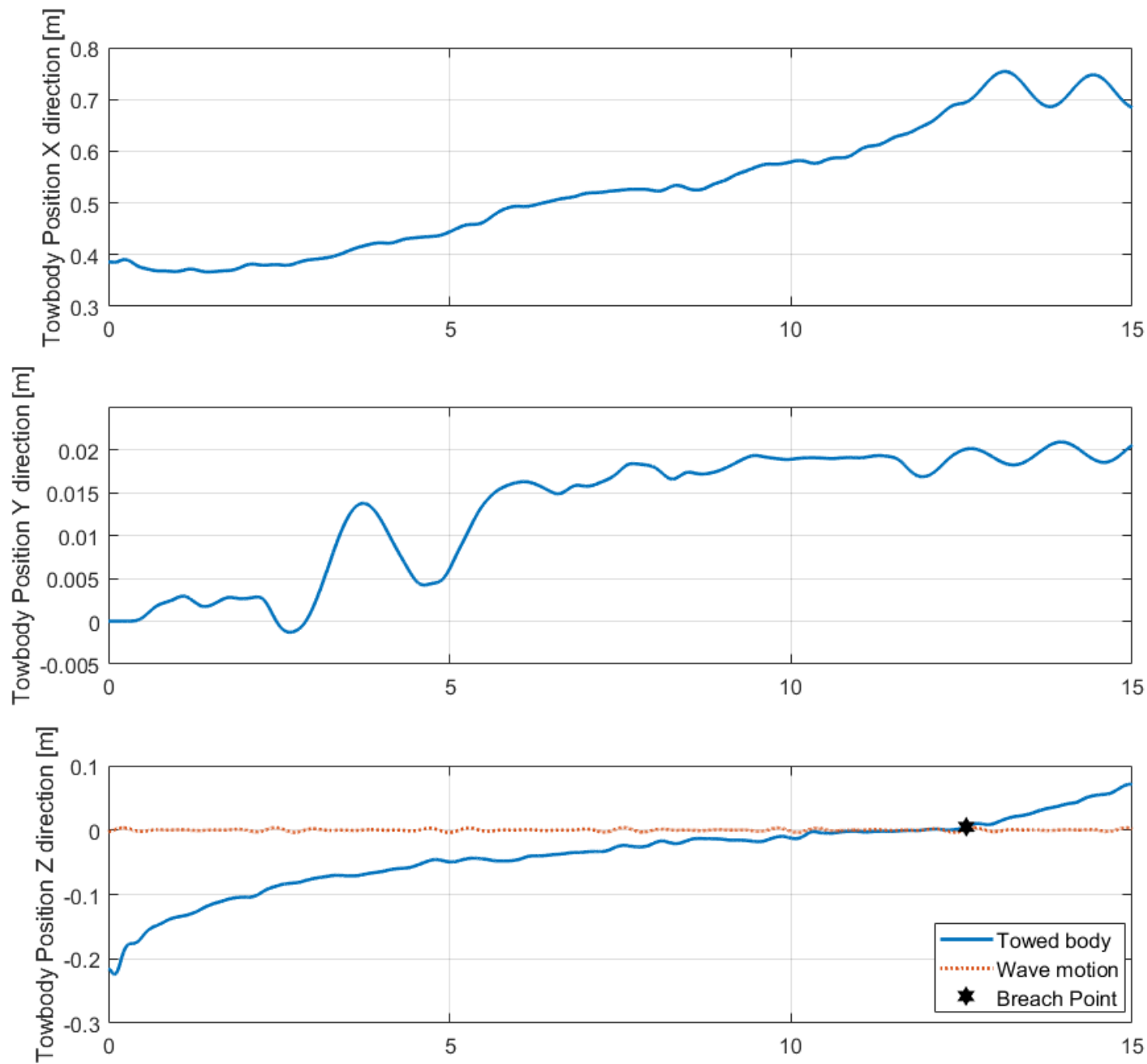

Figure A.6: Simulated results for the towed body position with only heave compensation during a recovery operation with irregular waves at SS6. The towed body breach point is indicated by a hexagram. 


\section{Appendix B}

\section{Technical Drawings}

Appendix B contains the technical drawing package for the ship motion simulator. Changes to the design were made ad hoc during assembly to accommodate additional equipment which are not reflected in the drawings below. 


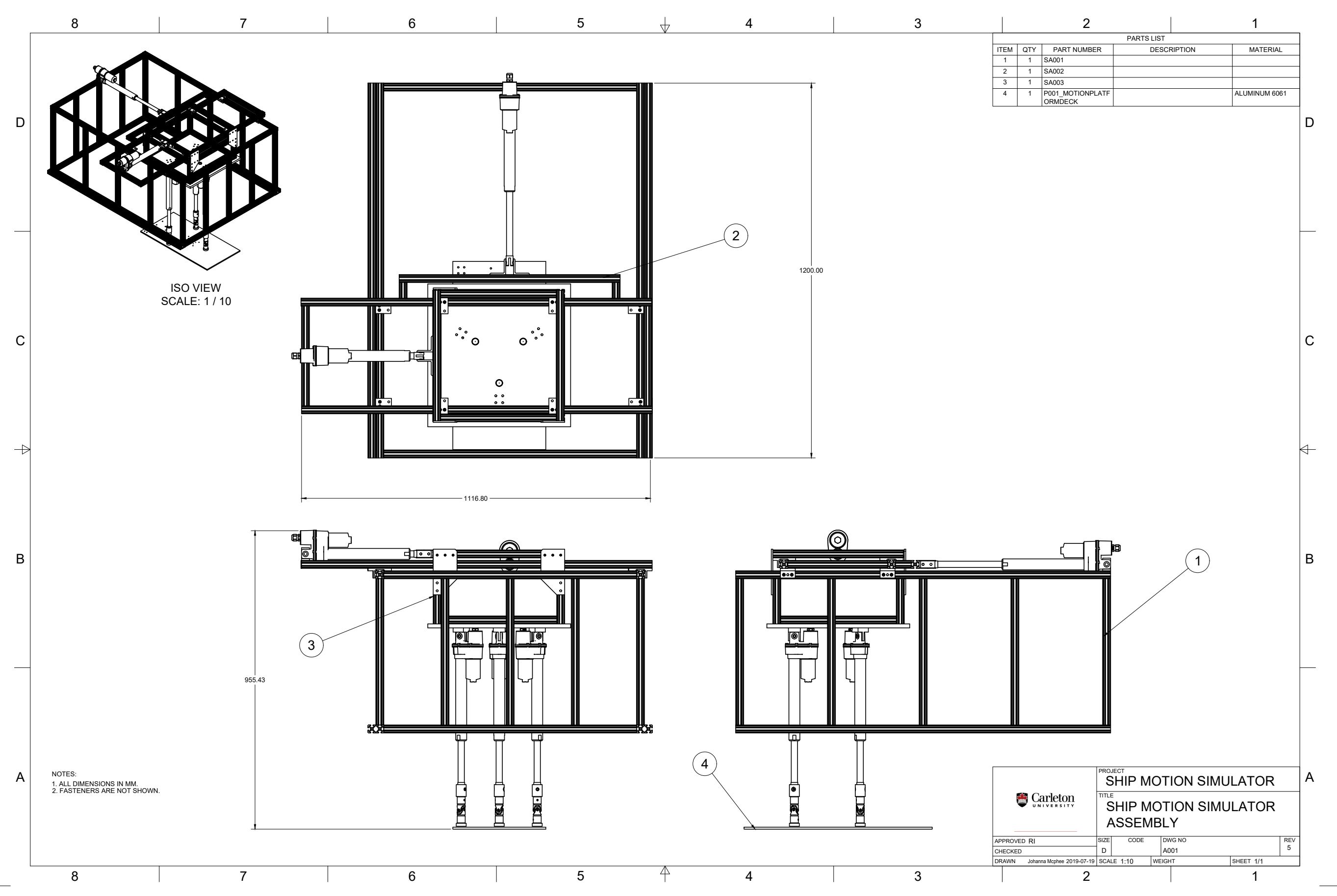




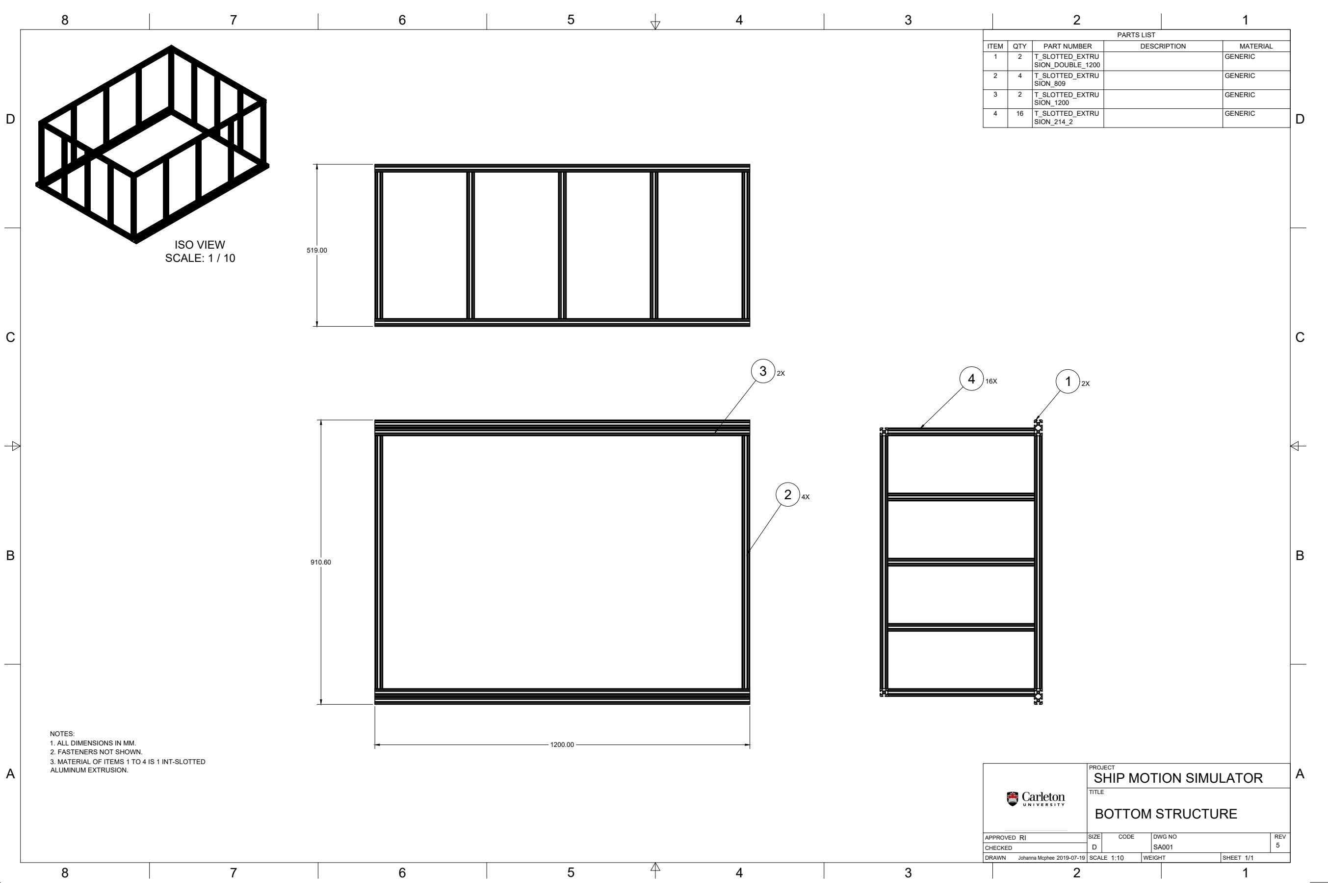




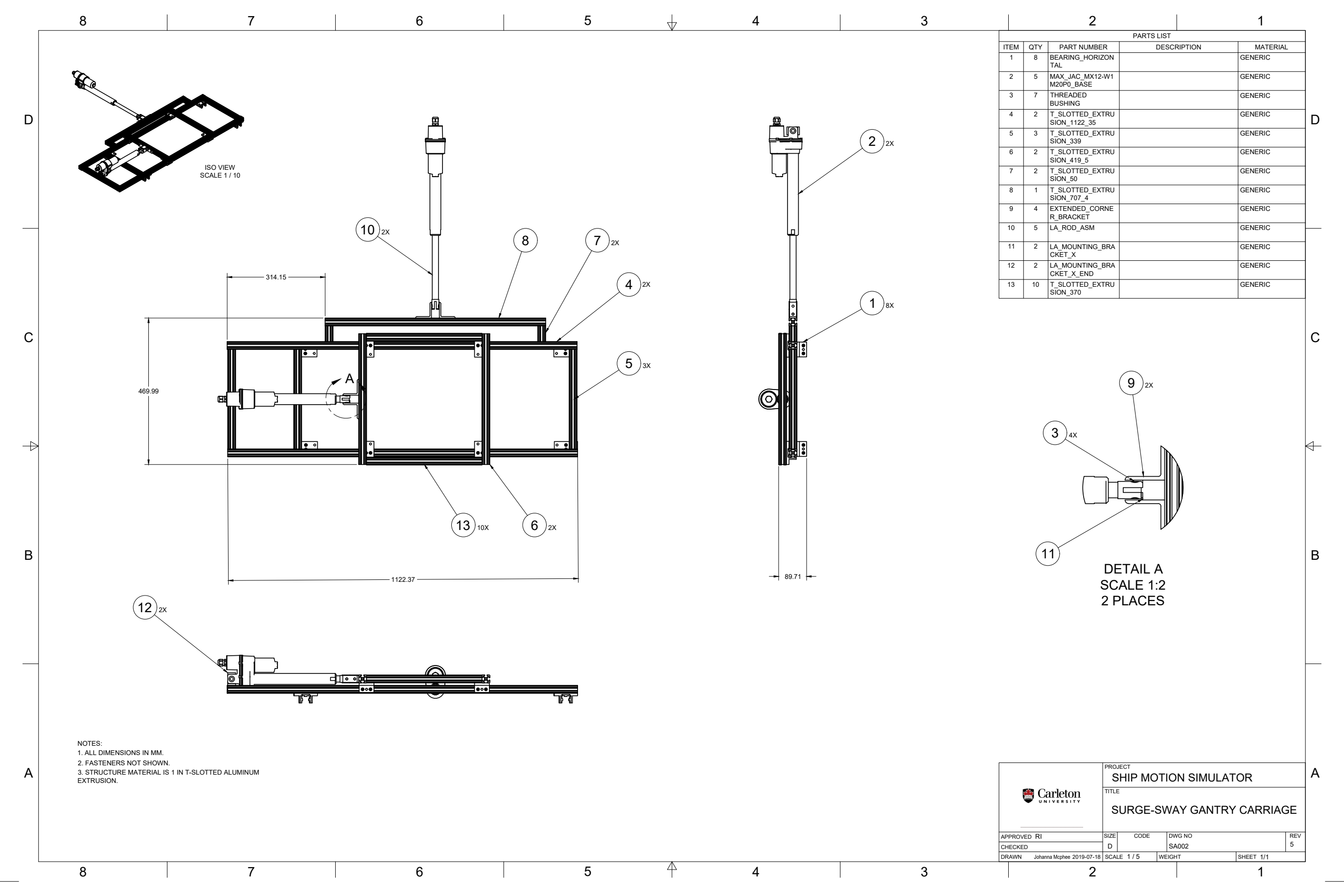




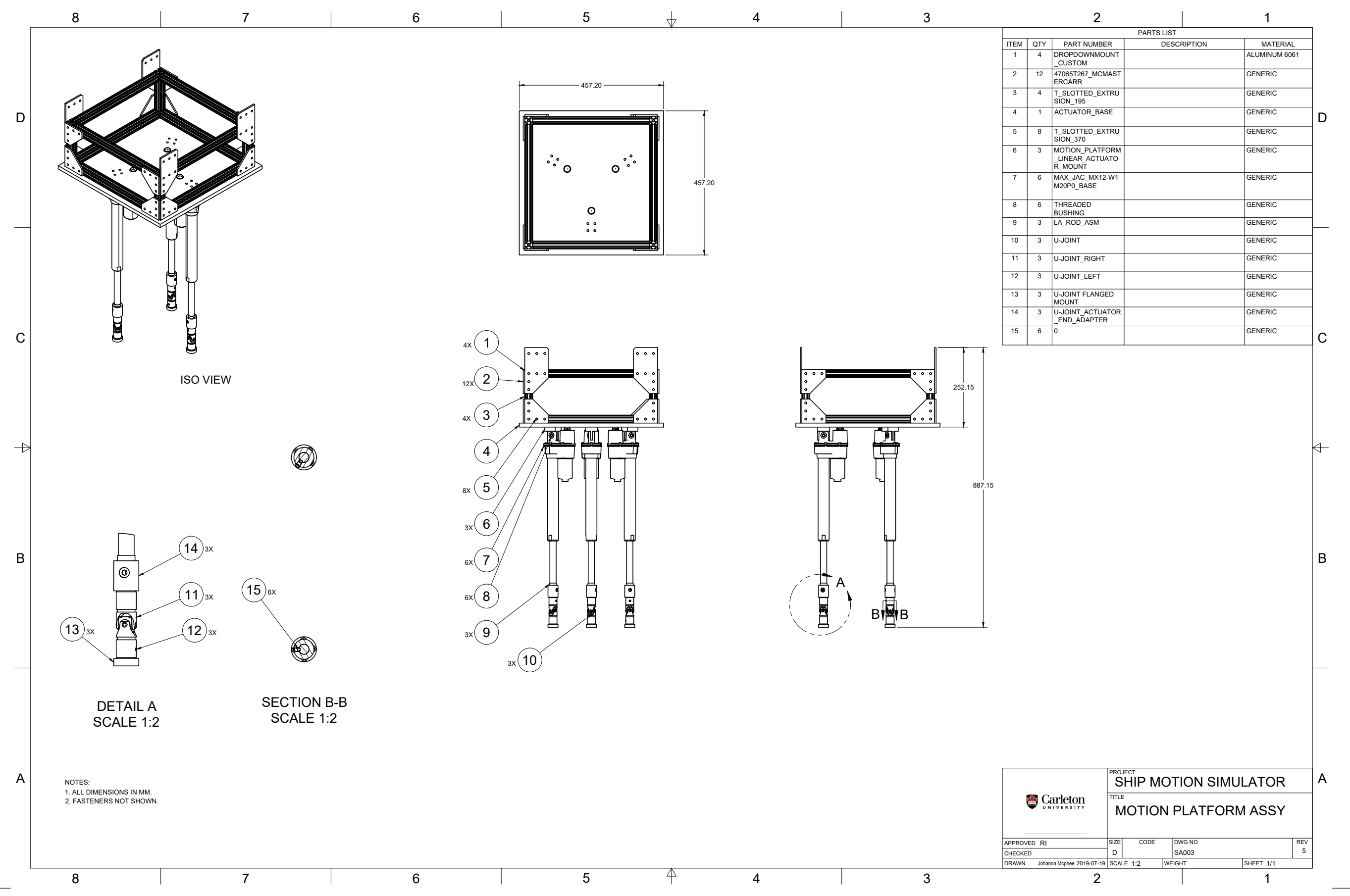




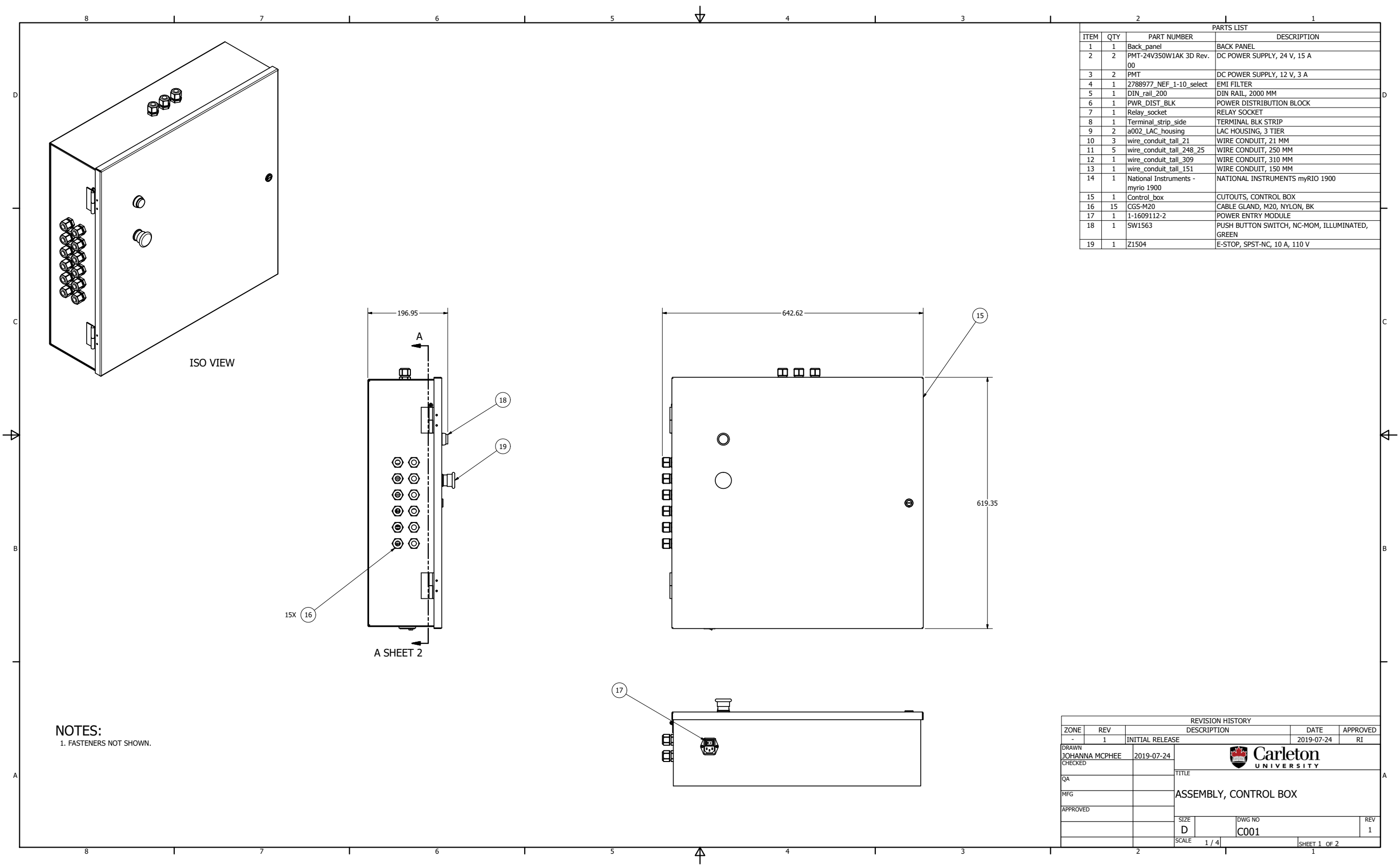




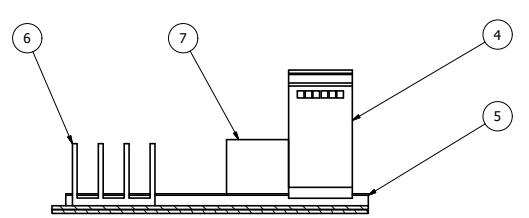

SECTION B-B REMOVED AND ROTATED 90 DEG

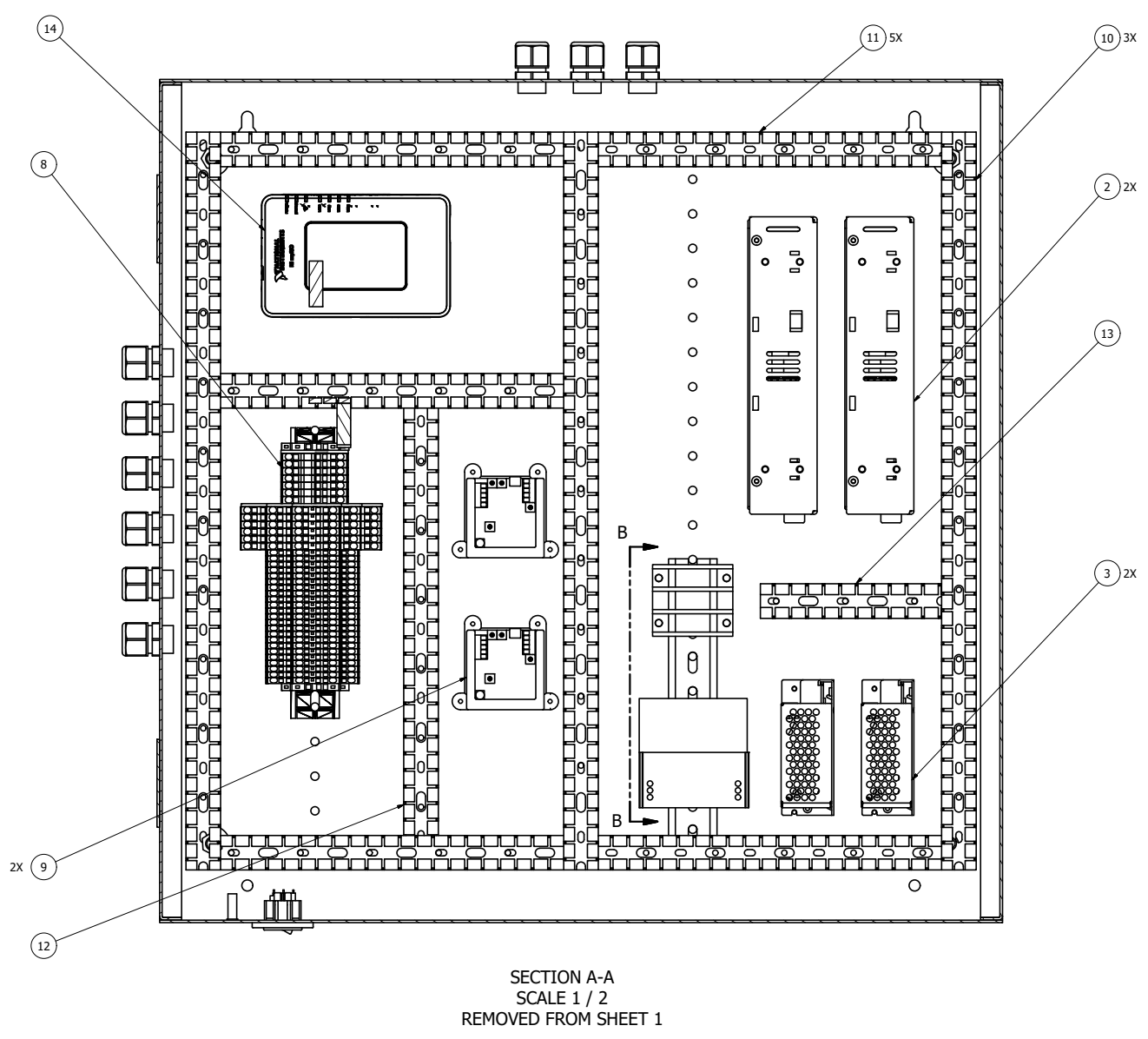




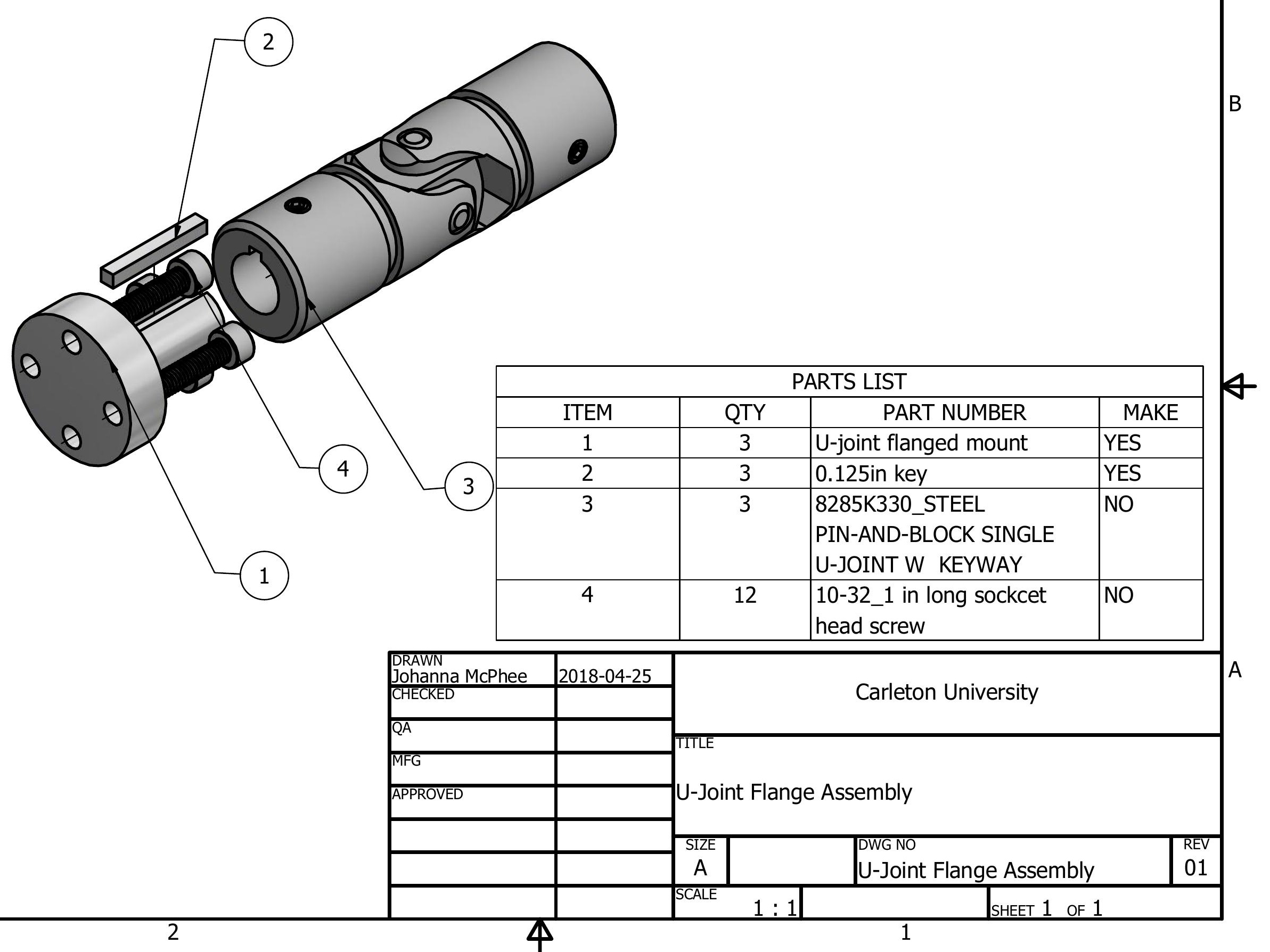



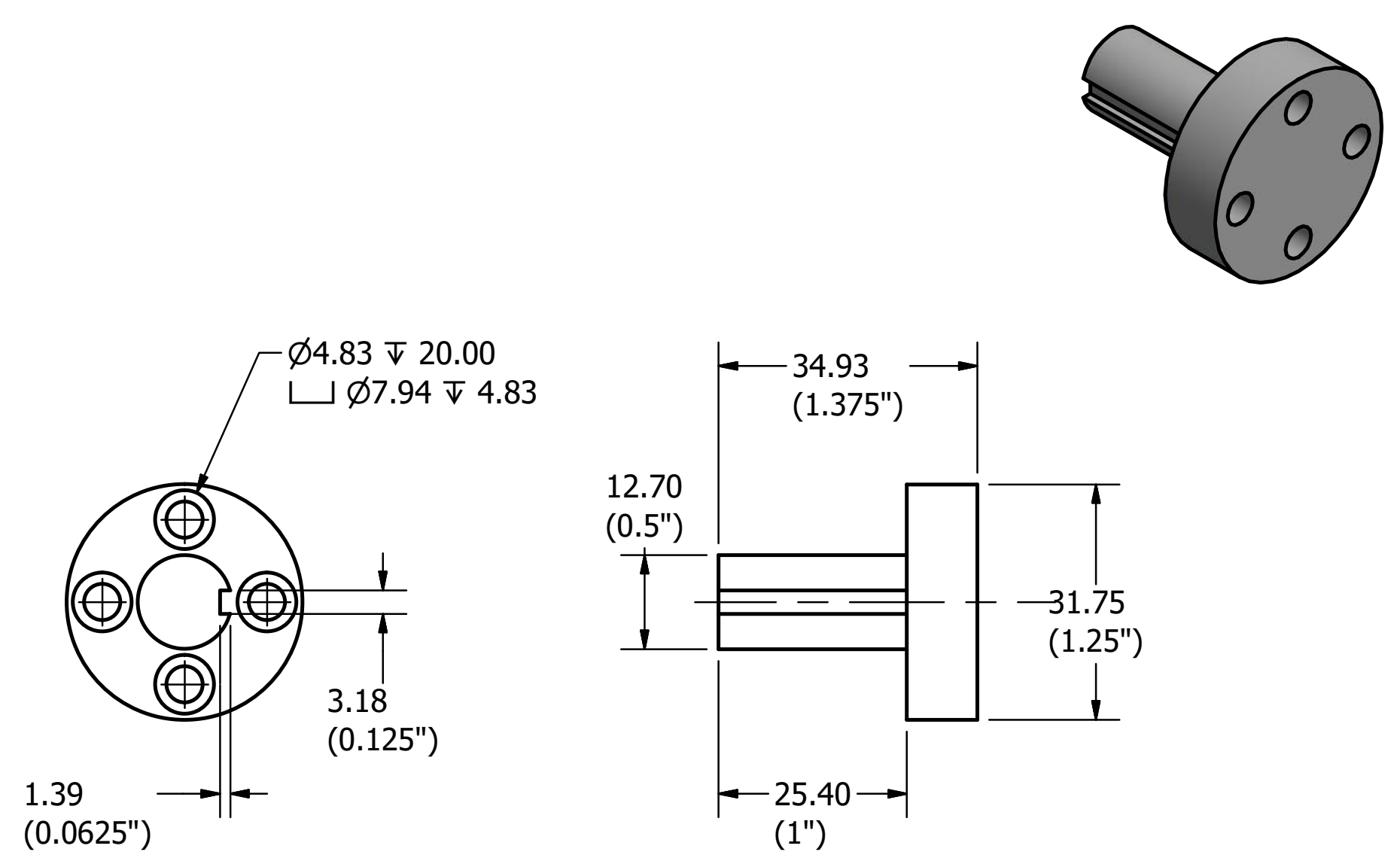

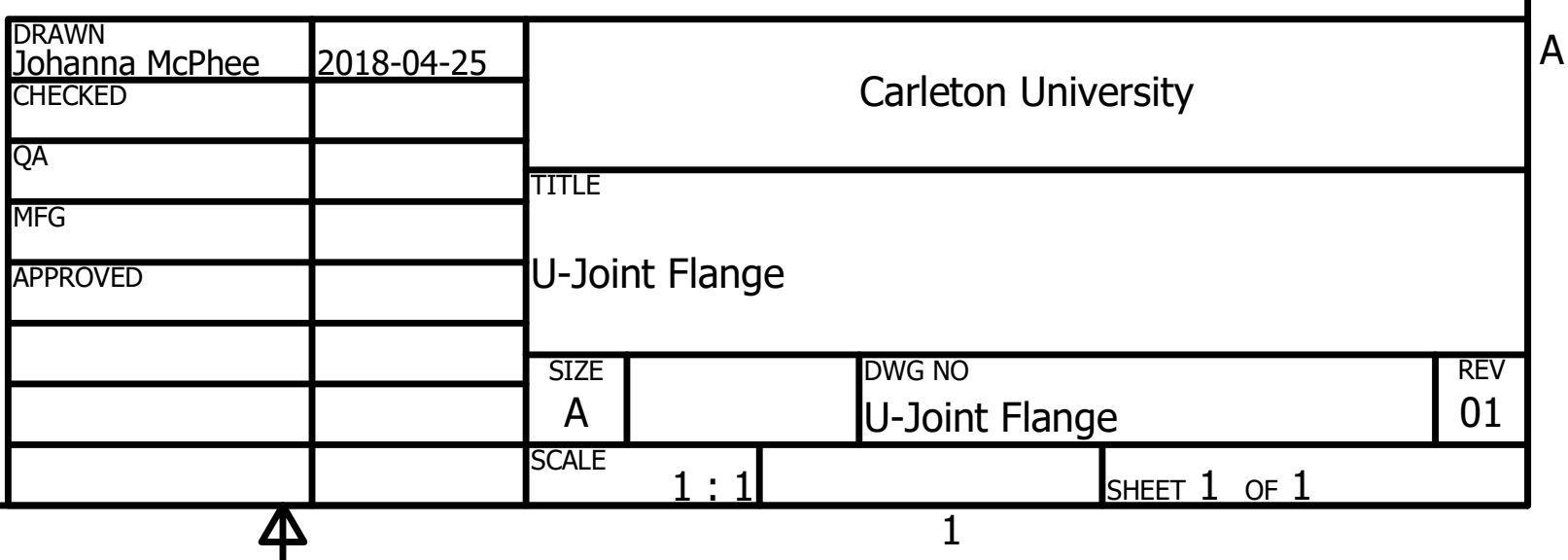




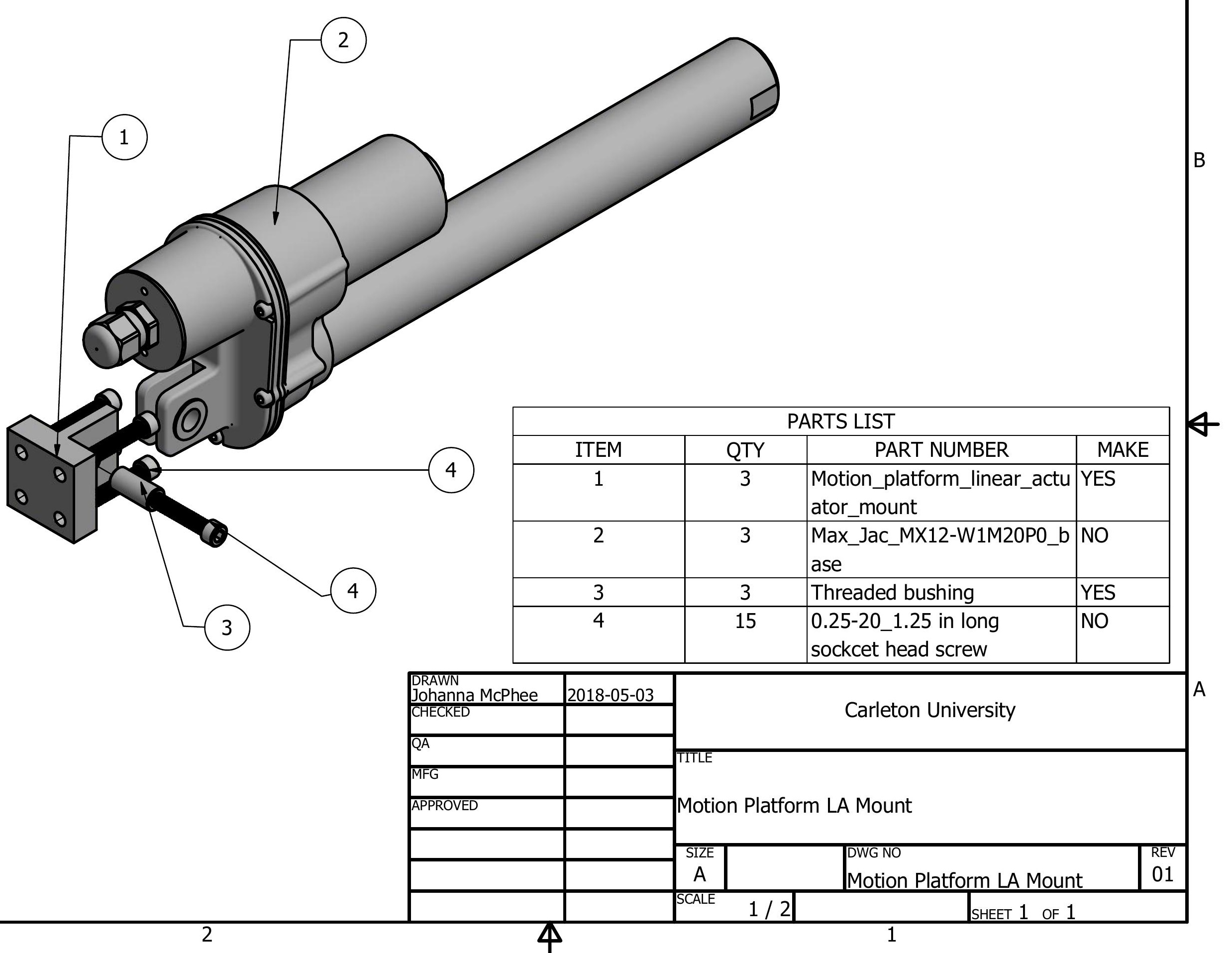



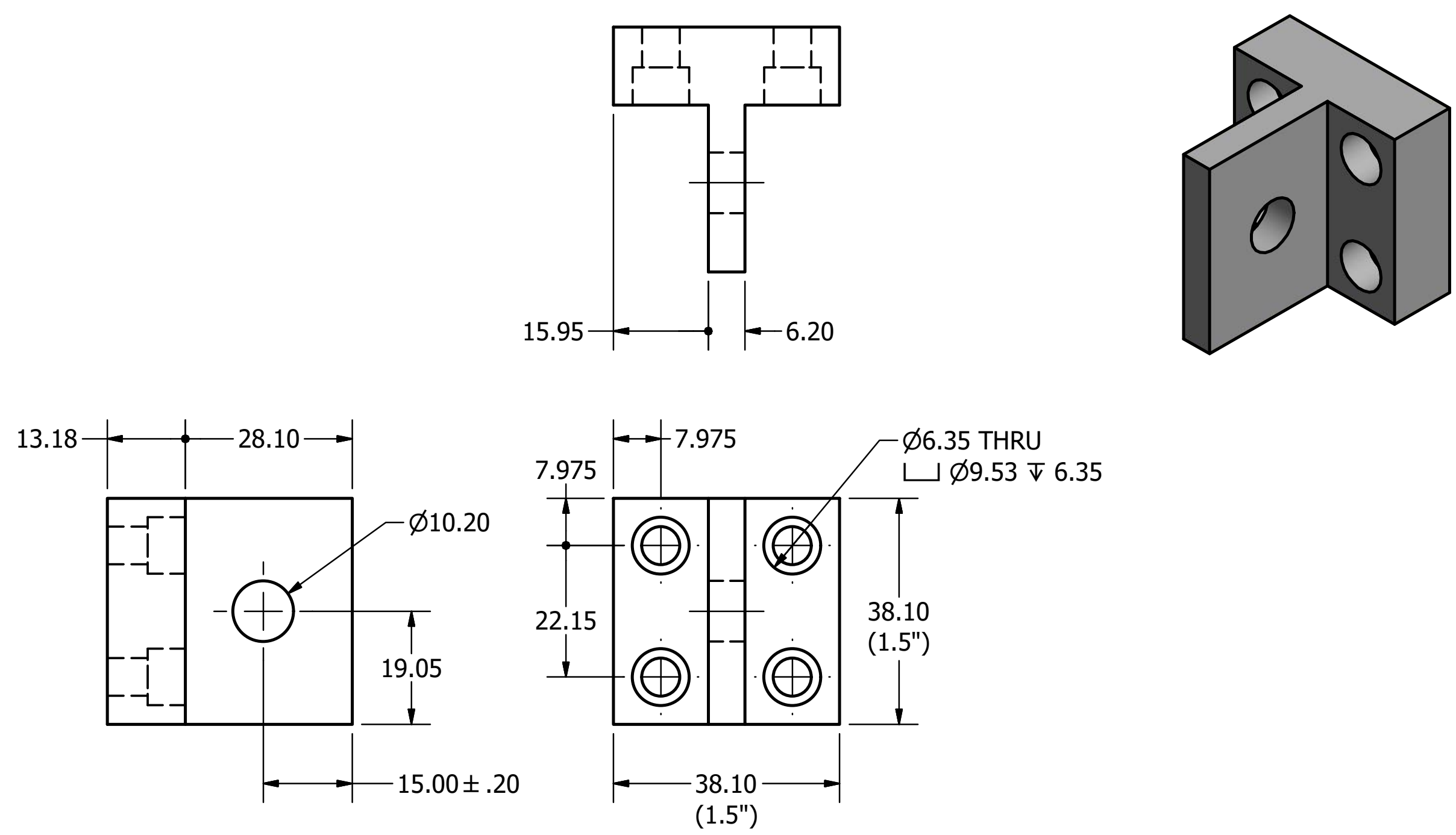

B
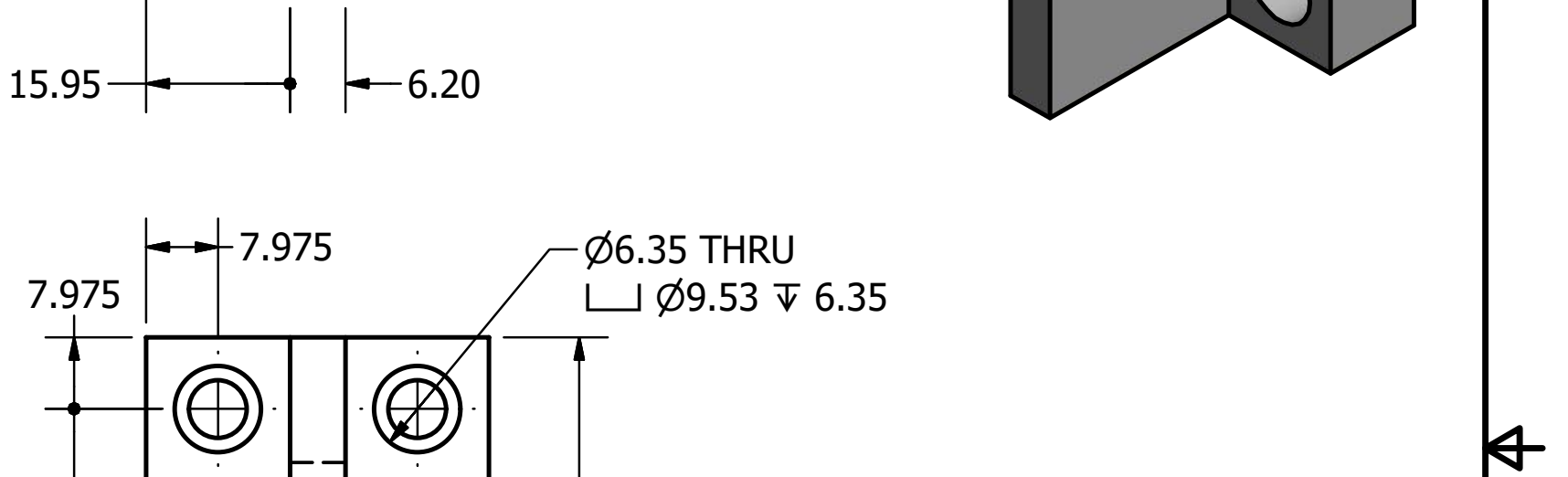

\begin{tabular}{|c|c|c|c|c|}
\hline \begin{tabular}{|l} 
DRAWN \\
Johanna McPhee \\
CHECKED \\
\end{tabular} & 2018-05-03 & \multicolumn{3}{|c|}{ Carleton University } \\
\hline QA & & \multirow{2}{*}{ TITLE } & & \\
\hline NFG & & & & \\
\hline \begin{tabular}{|l} 
APPROVED \\
\end{tabular} & & \multicolumn{3}{|c|}{ Motion Platform LA Mount } \\
\hline & & \multirow{2}{*}{$\begin{array}{c}\text { SIZE } \\
\mathrm{A}\end{array}$} & DWG NO & REV \\
\hline & & & Motion_platform_linear_actuato & Pito \\
\hline & & SCALE & SHEET 1 OF 1 & \\
\hline
\end{tabular}



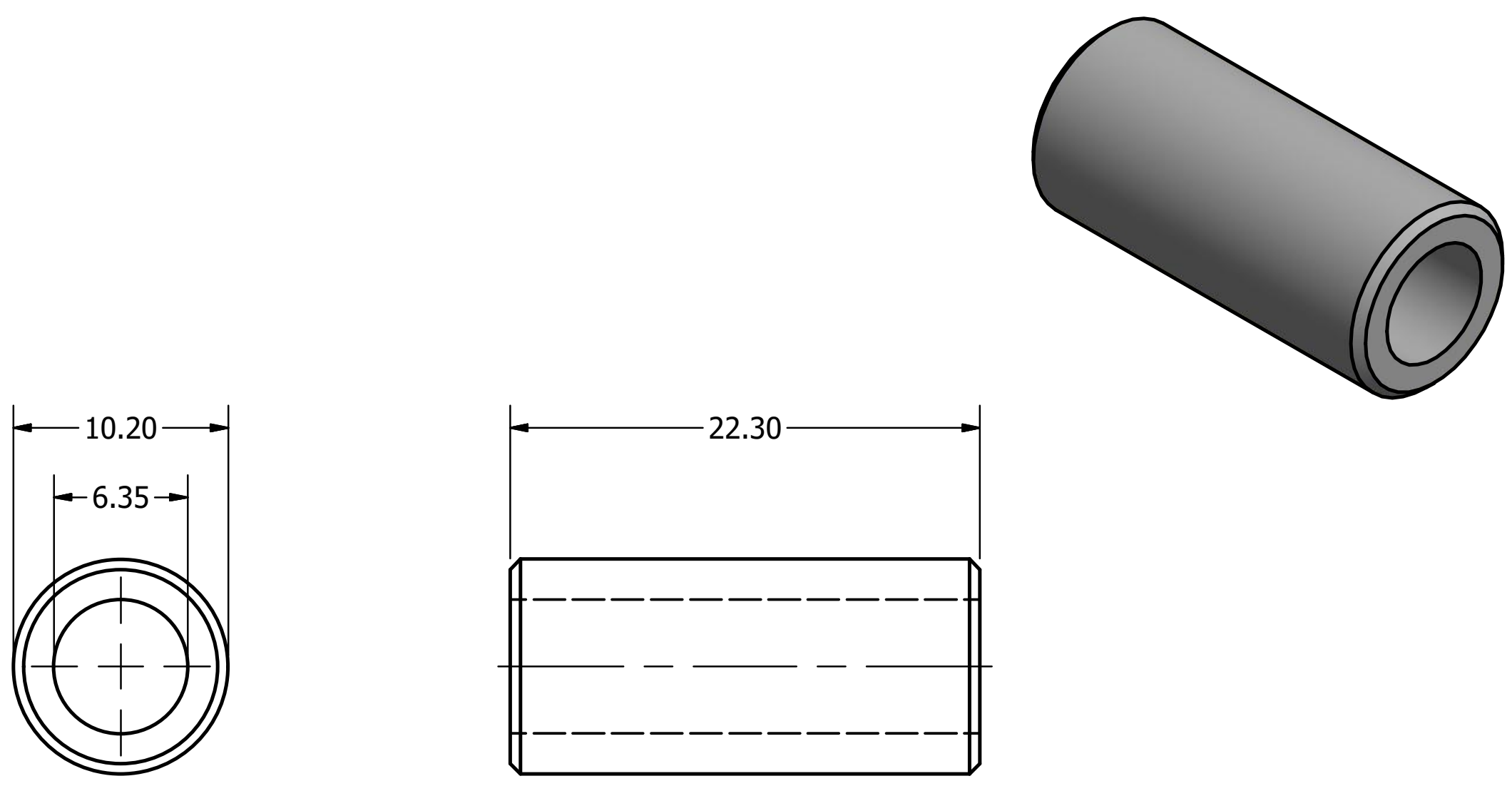

All dimensions in $\mathrm{mm}$.

Material: 1/2" aluminum round sto $\mathrm{k}$

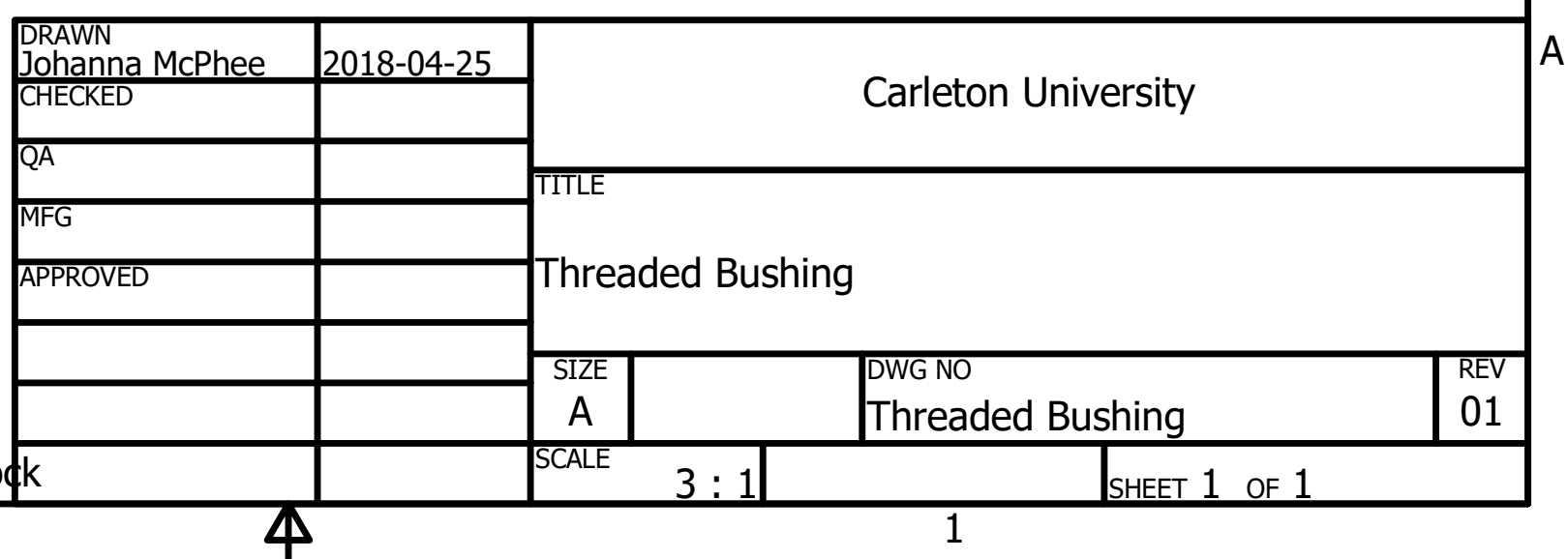

